US Army Corps

of Engineers ${ }_{\circledast}$

Engineer Research and

Development Center

Wetlands Regulatory Assistance Program

Hydrogeomorphic (HGM) Approach to Assessing Wetland Functions: Guidelines for Developing Guidebooks (Version 2)

R. Daniel Smith, Chris V. Noble, and Jacob F. Berkowitz

June 2013 
The US Army Engineer Research and Development Center (ERDC) solves the nation's toughest engineering and environmental challenges. ERDC develops innovative solutions in civil and military engineering, geospatial sciences, water resources, and environmental sciences for the Army, the Department of Defense, civilian agencies, and our nation's public good. Find out more at www.erdc.usace.army.mil.

To search for other technical reports published by ERDC, visit the ERDC online library at http://acwc.sdp.sirsi.net/client/default. 


\section{Hydrogeomorphic (HGM) Approach to Assessing Wetland Functions: Guidelines for Developing Guidebooks (Version 2)}

R. Daniel Smith, Chris V. Noble, and Jacob F. Berkowitz

Environmental Laboratory

U.S. Army Engineer Research and Development Center

3909 Halls Ferry Rd.

Vicksburg, MS 39180-6199

Final report

Approved for public release; distribution is unlimited.

Prepared for U.S. Army Corps of Engineers

Washington, DC 20314-1000 


\section{Abstract}

The Hydrogeomorphic (HGM) Approach for assessing wetlands was developed by the U.S. Army Corps of Engineers as a procedure for assessing the capacity of a wetland to perform ecological functions. The Approach requires classification of wetlands based on geomorphic setting, water source, and hydrodynamics. The objective of the Guidelines for Guidebook Development is to provide detailed guidance on methods and procedures that have proven helpful in developing existing guidebooks. This document contains an overview of the HGM Approach; how to classify and characterize wetland subclasses; developing assessment models; selecting reference wetlands and managing reference wetland data; testing and calibrating models; developing assessment protocols for using assessments; validating assessment models; and assessment application examples.

DISCLAIMER: The contents of this report are not to be used for advertising, publication, or promotional purposes. Citation of trade names does not constitute an official endorsement or approval of the use of such commercial products. All product names and trademarks cited are the property of their respective owners. The findings of this report are not to be construed as an official Department of the Army position unless so designated by other authorized documents. 


\section{Contents}

Abstract...................................................................................................................................... if

Figures and Tables............................................................................................................vi

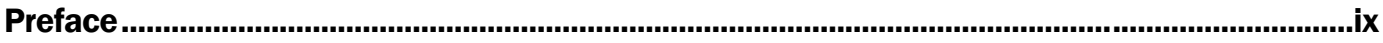

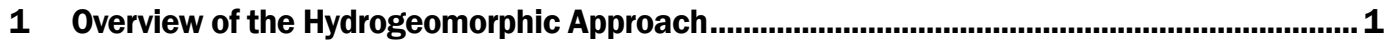

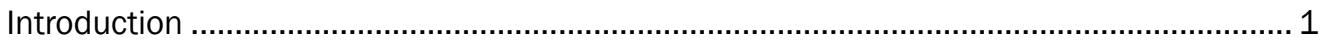

Wetland assessment and the 404 Regulatory Program ............................................... 3

Programmatic and technical criteria .............................................................................. 7

Criteria 1: Applicable to all wetland types ............................................................................. 7

Criteria 2: Compatible with time and resources available for 404 Permit review................... 8

Criteria 3: Documented, technically defensible, and compatible ........................................ 12

Overview of the development phase ............................................................................. 12

Task 1: Organize assessment team ............................................................................ 12

Task 2: Classify and characterize wetland subclass........................................................ 14

Task 3: Develop conceptual assessment models .............................................................. 15

Task 4: Peer review of pre-calibrated draft guidebook ..................................................... 17

Task 5: Select and sample reference wetlands ................................................................ 20

Task 6: Test and calibrate assessment variables and verify assessment models................. 22

Task 7: Conduct peer review and field testing of calibrated draft guidebook ....................... 23

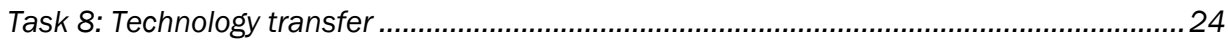

2 Classify and Characterize Wetland Subclasses...................................................................26

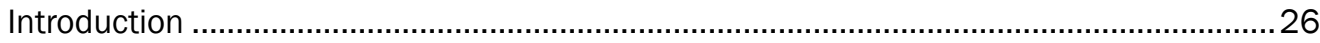

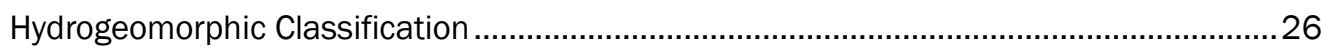

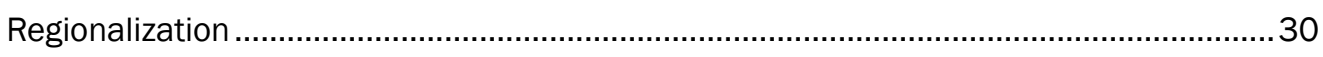

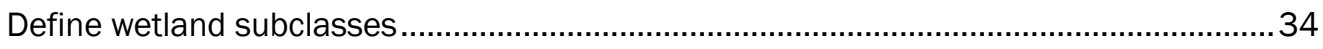

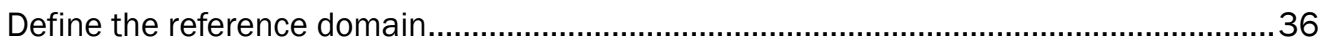

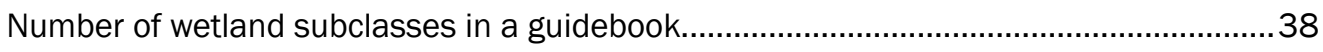

Characterize the wetland subclass ........................................................................ 41

Climate

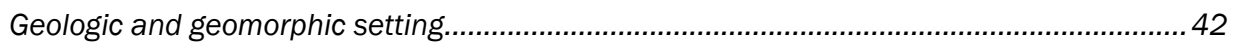

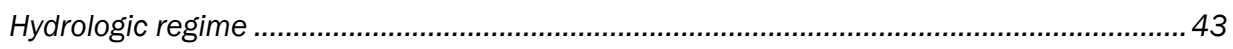

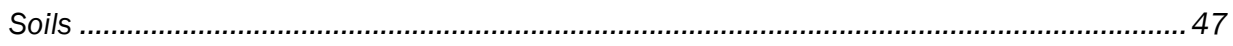

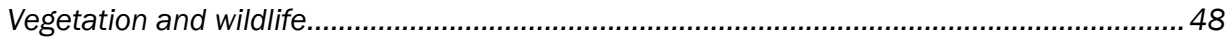

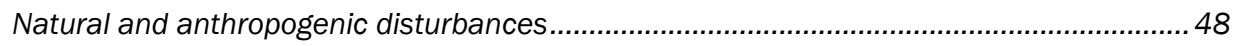

3 Conceptualize Assessment Models ........................................................................51

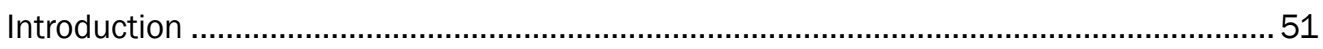

Overview of assessment models ............................................................................. 51

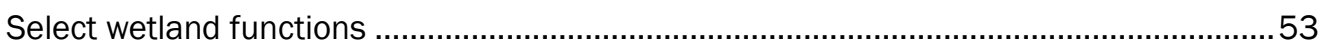

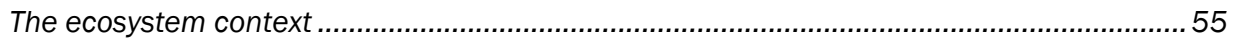




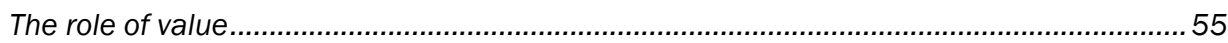

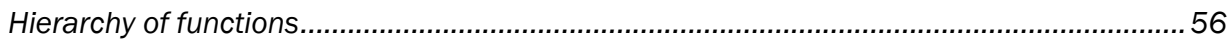

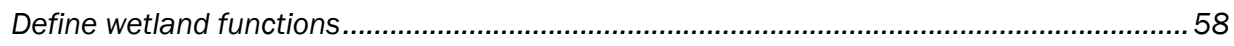

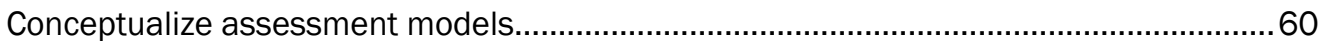

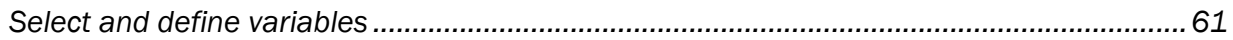

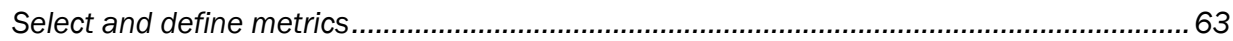

Define the relationship between variables and functional capacity.....................................65

Define the relationship between variables ......................................................................6 67

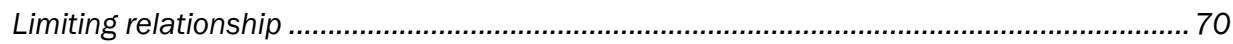

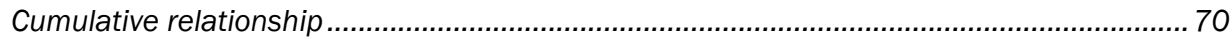

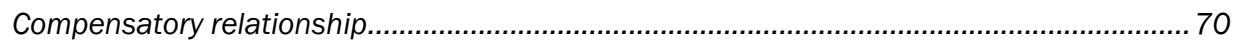

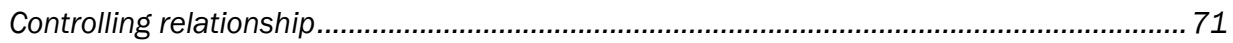

4 Reference Wetlands and Reference Wetland Data ...........................................................72

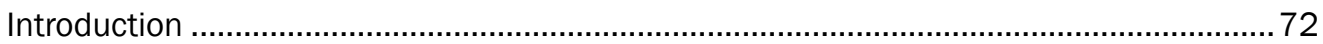

Reference wetland definitions.................................................................................... 72

Purpose of reference wetlands................................................................................. 73

Select reference wetlands ......................................................................................... 73

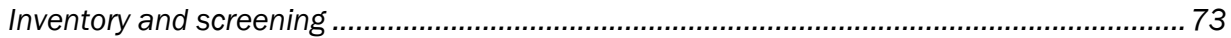

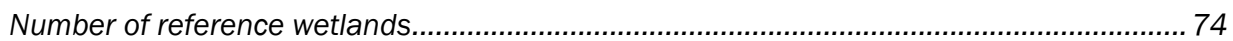

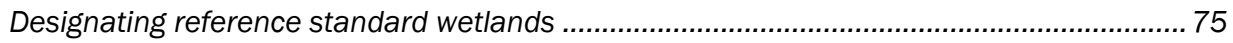

Prepare to collect reference wetland data................................................................... 76

Variable documentation.......................................................................................................... 76

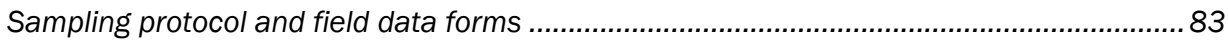

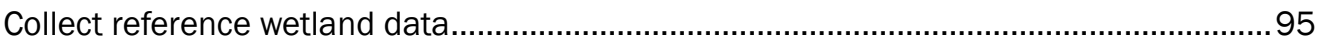

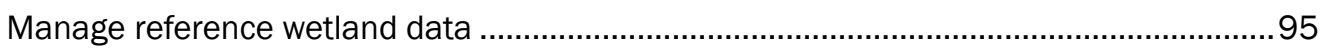

5 Calibrate, Verify, and Field-Test Assessment Models ............................................................98

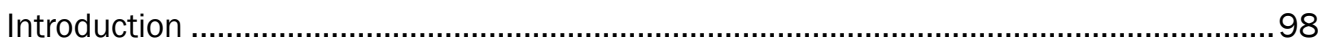

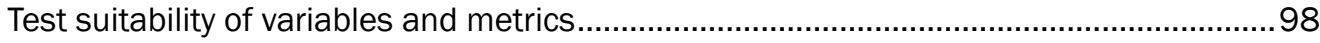

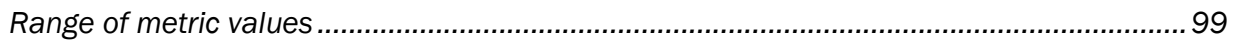

Correlation between variables....................................................................................... 102

Calibrate metric values .............................................................................................. 104

Verify assessment models ................................................................................ 107

Sensitivity analysis ............................................................................................ 108

Test assessment models with sample data sets.............................................................112

Field-test assessment models......................................................................... 112

Generic procedure for field-testing assessment models .................................................114

Evaluate temporal consistency ..................................................................................... 121

6 Develop End-User Assessment Protocol................................................................................... 123

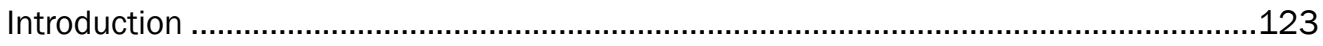

Define assessment objectives..........................................................................123

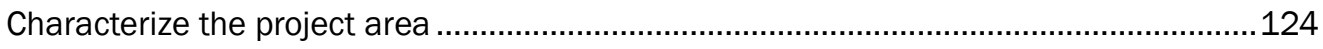

Screen for red flags .............................................................................................. 125

Define the wetland assessment area ........................................................................125

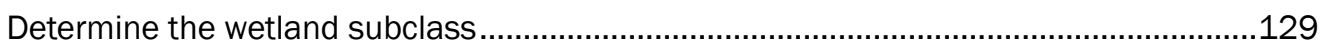




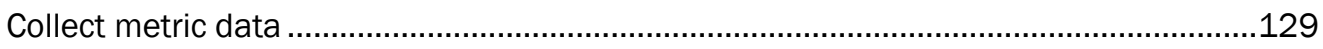

Analyze the data ................................................................................................. 133

Apply assessment results ............................................................................. 134

7 Validate Assessment Models........................................................................................... 135

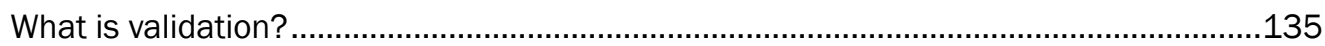

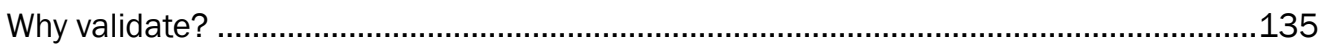

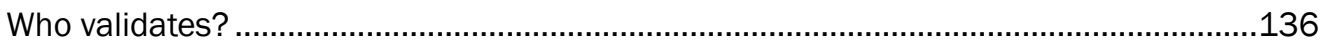

Approaches to validate assessment models ..................................................................136

Relationship between the $\mathrm{FCl}$ and independent measure of function.............................137

Testing the assessment model versus assessment model components ........................140

A generic procedure for validating assessment models ................................................142

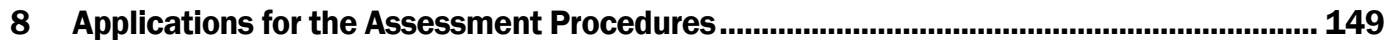

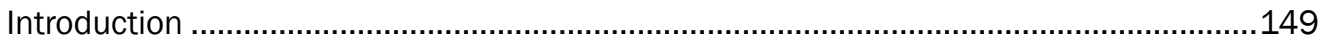

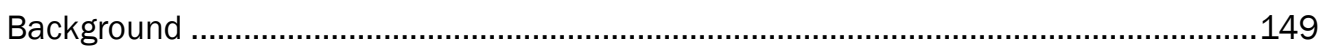

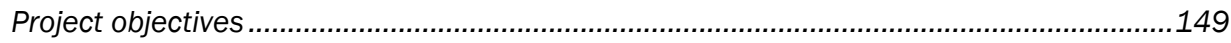

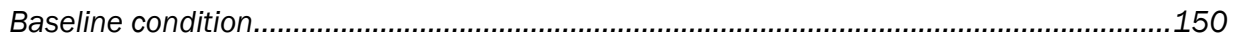

Comparing multiple wetlands of the same subclass .........................................................150

Compute potential project impacts..................................................................................150

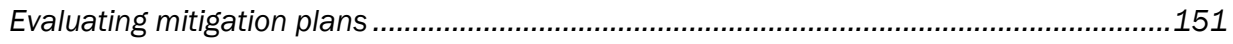

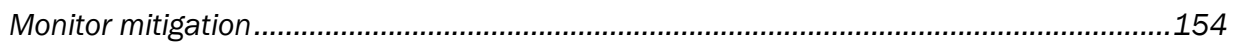

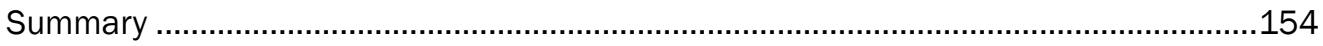

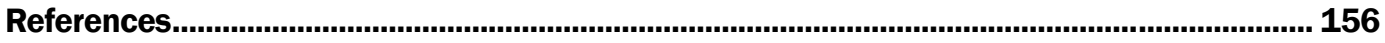

Report Documentation Page 


\section{Figures and Tables}

\section{Figures}

Figure 1. Phases of the HGM Approach.....

Figure 2. Possible flow paths of rainfall on hill slopes, including (1) infiltration-excess overland flow; (2) groundwater recharge; (3) throughflow; (4) return flow; and (5) saturation overland flow.

Figure 3. Example of Level III and Level IV U.S. Ecoregions for Wisconsin.

Figure 4. Example of an ecological unit at the Province level of the U.S. Forest Service national regionalization system.

Figure 5. Example of Land Resource Regions (pink) and Major Land Resource Areas (purple).

Figure 6. Reference domain for the Delta Region of Arkansas (Klimas et al. 2004). ...................... 37

Figure 7. EPA Level III Ecoregion 73 (Mississippi Alluvial Plain)...................................................... 37

Figure 8. Key to the Wetland Classes in the Coastal Plain region of Arkansas (Klimas et al. 2005)......

Figure 9. Key to the wetland subclasses and community types in the Coastal Plain region of Arkansas (Klimas et al. 2005) (continued).

Figure 10. Generalized landscape position of Headwater Slope wetlands in southeast Mississippi and southwest Alabama (Noble et al. 2007).

Figure 11. Typical form and locations of geomorphic and man-made features within the Arkansas Valley Wetland Planning Region (Klimas et al. 2008).

Figure 12. Typical landscape positions of depression, fringe, and slope wetlands in the Arkansas Valley Wetland Planning Region (Klimas et al. 2008)...

Figure 13. Relationship between hypothetical metric values and the variable subindex.

Figure 14. The hierarchy of wetland functions.

Figure 15. Relationship between tree basal area metric and functional capacity expressed as a subindex.

Figure 16. Relationship between the soil type metric and functional capacity expressed as a subindex.

Figure 17. Effects of various mathematical operations in combining $V_{A}$ and $V_{B}$. In all cases, the value of $V_{B}$ is fixed at 0.1 , while $V_{A}$ varies between 0 and 1.0.

Figure 18. Trend in variance of a metric. ......................................................................................... 75

Figure 19. Example of a Reference Wetland Documentation form................................................ 78

Figure 20. Example of a variable documentation form............................................................ 80

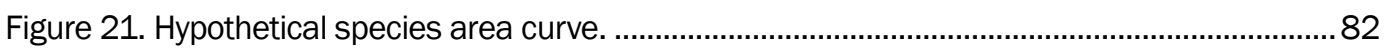

Figure 22. Number of samples versus average metric value .......................................................... 82

Figure 23. Example Sampling Protocol (continued).................................................................. 86

Figure 24. Example Field Data Form for summarizing assessment variable metric values (continued).

Figure 25. Scatter graph of a narrow range of hypothetical metric values and samples sorted by ascending metric values. 
Figure 26. Scatter graph of a wider range of hypothetical metric values and samples sorted by ascending metric values.

Figure 27. Scatter graph of a tree basal area metric values and sample number (sorted ascending).

Figure 28. Hypothetical metric value in relation to disturbance categories. 101

Figure 29. Tree basal area metric value in relation to disturbance categories.

Figure 30. Metric values and correlation matrix for the variables $V_{\text {FREQ }}, V_{S L O P E}$, and $V_{\text {WIDTH. }}$

Figure 31. Subindex values and correlation matrices for the model variables $V_{\text {FREQ }}$, VSLOPE, $V_{\text {ROUGH, and }} \mathrm{VWIDTH}$ 104

Figure 32. Subindex in relation to tree basal area metric values. 105

Figure 33. Regression of tree basal area against disturbance in moderately disturbed reference wetlands.

Figure 34. Subindices in relation to woody debris metric values.

Figure 35. Sensitivity analysis for the assessment model FCl $=\left(\left(V_{\text {FREQ }}+\left(V_{\text {LITTER }}+\right.\right.\right.$ $\left.\left.V_{(w D)} / 2\right) / 2\right)^{1 / 2}$.

Figure 36. Tabular and graphical display of sensitivity results for the simple three-variable assessment model $\mathrm{FCl}=\left(\left(V_{F R E Q}+\left(V_{L I T E R}+V_{C W D}\right) / 2\right) / 2\right)^{1 / 2}$. The higher initial response and increased slope observed for $V_{\text {FREQ }}(A)$ indicate that the variable maintains a stronger impact on $\mathrm{FCl}$ scores compared to $V_{\text {LITTER }}+V_{\text {CWD }}$ (B). Note that $V_{\text {LITER }}$ and $V_{\text {CWD }}$ receive equal treatment in the model, resulting in identical graphs.

Figure 37. Example field-tester evaluation form................................................................... 117

Figure 38. Results of field tests of assessment models for two functions at one field site............120

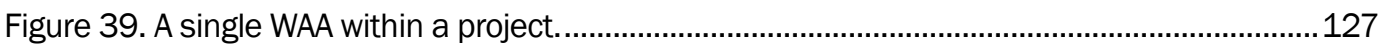

Figure 40. Spatially separated WAAs from the same regional subclass within a project. ..............127

Figure 41. More than one regional subclass within a project area. ...............................................127

Figure 42. PWAAs defined on the basis of differences in site-specific characteristics. ..................127

Figure 43. Recent logging in a portion of a headwater slope wetland...........................................128

Figure 44. Example application of geomorphic mapping and aerial photography to develop a preliminary wetland classification for a proposed project area. .....................................130

Figure 45. Field data collection checklist. .............................................................................. 131

Figure 46. Sample plot layout for riverine wetlands in Western Tennessee...................................131

Figure 47. Expected relationship between the modeled Functional Capacity Index (FCl) and the independent measurement of function.

Figure 48. Relationship between the $\mathrm{FCl}$ and an independent measure of function (i.e., number of species of breeding amphibians captured in each wetland).

Figure 49. Hypothetical relationship between percent leaf litter cover (i.e., metric) and an average value of total carbon exported from a number of riverine wetlands (i.e., the independent measure of function where the solid line represents the expected relationship based on the assessment model.

\section{Tables}

Table 1. Mitigation ratios and potential FCl for Low Gradient Riverine Backwater Wetlands in the Southeast.

Table 2. Tasks in developing HGM Guidebooks. 


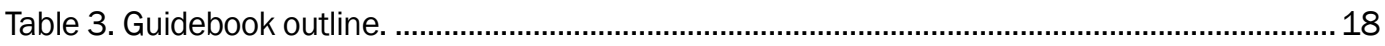

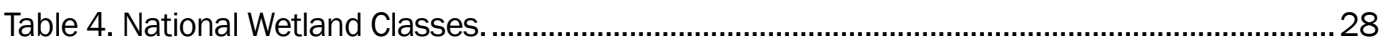

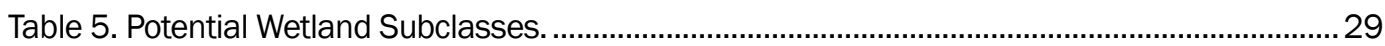

Table 6. Wetland functions and potential independent measures of function.................................60

Table 7. Permissible mathematical relations and operations for various scales of

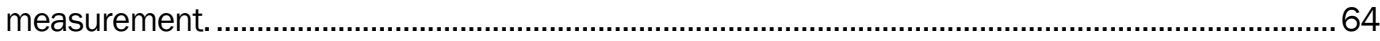

Table 8. Types of interactions between model variables, and their mathematical expression, that may be useful in developing assessment models for the HGM Approach............. 69

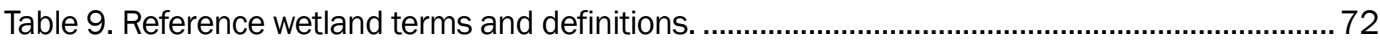

Table 10. Reference wetland inventory form ...............................................................................74

Table 11. Conditions for assigning disturbance rank to hydrologic variables (Adapted from

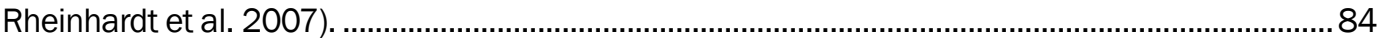

Table 12. Example of a spreadsheet for calculating tree basal area................................................9. 92

Table 13. Example of a spreadsheet for woody debris and log volume metric value

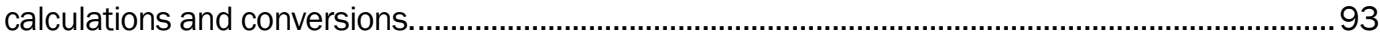

Table 16. Sequence of steps in a generic field-test procedure.........................................................114

Table 17. Comparison of field tester results at one field site......................................................119

Table 18. Field test for temporal consistency of one function......................................................122

Table 19. Red flag features and respective program or agency authority.......................................126

Table 21. Generic procedure for validating assessment models. .................................................142

Table 22. Comparison of baseline wetland FCl to Reference FCl scores. .......................................150

Table 23. Comparison of FCl scores between wetlands of the same subclass ............................. 151

Table 24. Example comparison of mitigation using HGM ..............................................................152 


\section{Preface}

This document was developed by the U.S. Army Engineer Research and Development Center (ERDC) at the request of Headquarters, U.S. Army Corps of Engineers (HQUSACE), with funding provided through the Wetlands Regulatory Assistance Program (WRAP). This document completes the document described in Hydrogeomorphic Approach to Assessing Wetland Functions: Guidelines for Developing Regional Guidebooks, Chapter 1 - Introduction and Overview of the Hydrogeomorphic Approach (Clairain 2002) and replaces published Chapters $1,3,4,7$, and 8. Comments should be submitted via the Internet at the following address: http://www.wes.army.mil/el/wetlands/hgmhp.html.

Written comments should be addressed to:

Department of the Army

Research and Development Center CEERD-EE-W

3909 Halls Ferry Road

Vicksburg, MS 39180-6199

This work took place under the general supervision of Patrick O'Brien, Chief, Wetlands and Coastal Ecology Branch; Dr. Edmond Russo, Chief, Ecosystem Evaluation and Engineering Division; Environmental Laboratory (EL), ERDC; and Dr. Elizabeth C. Fleming, Director, EL, ERDC.

COL Kevin J. Wilson was Commander of ERDC. Dr. Jeffery P. Holland was Director. 


\section{Overview of the Hydrogeomorphic Approach}

\section{Introduction}

The Hydrogeomorphic Approach to the assessment of wetland functions, hereafter referred to as the HGM Approach, is a formal two-step process for first developing procedures and then applying those procedures to assess wetland functions. More recently, the approach has been applied to the assessment of other types of ecosystems such as headwater streams (Noble et al. 2007). During the "development phase," assessment procedures are developed to assess the functions of a specific type of wetland in a specific geographic region. During the "application phase," the assessment procedures are used to assess wetland functions in a project-specific context (Figure 1). The development and application phases incorporate four fundamental components of the HGM Approach, including hydrogeomorphic classification, reference wetlands, assessment models, and end-user assessment protocols.

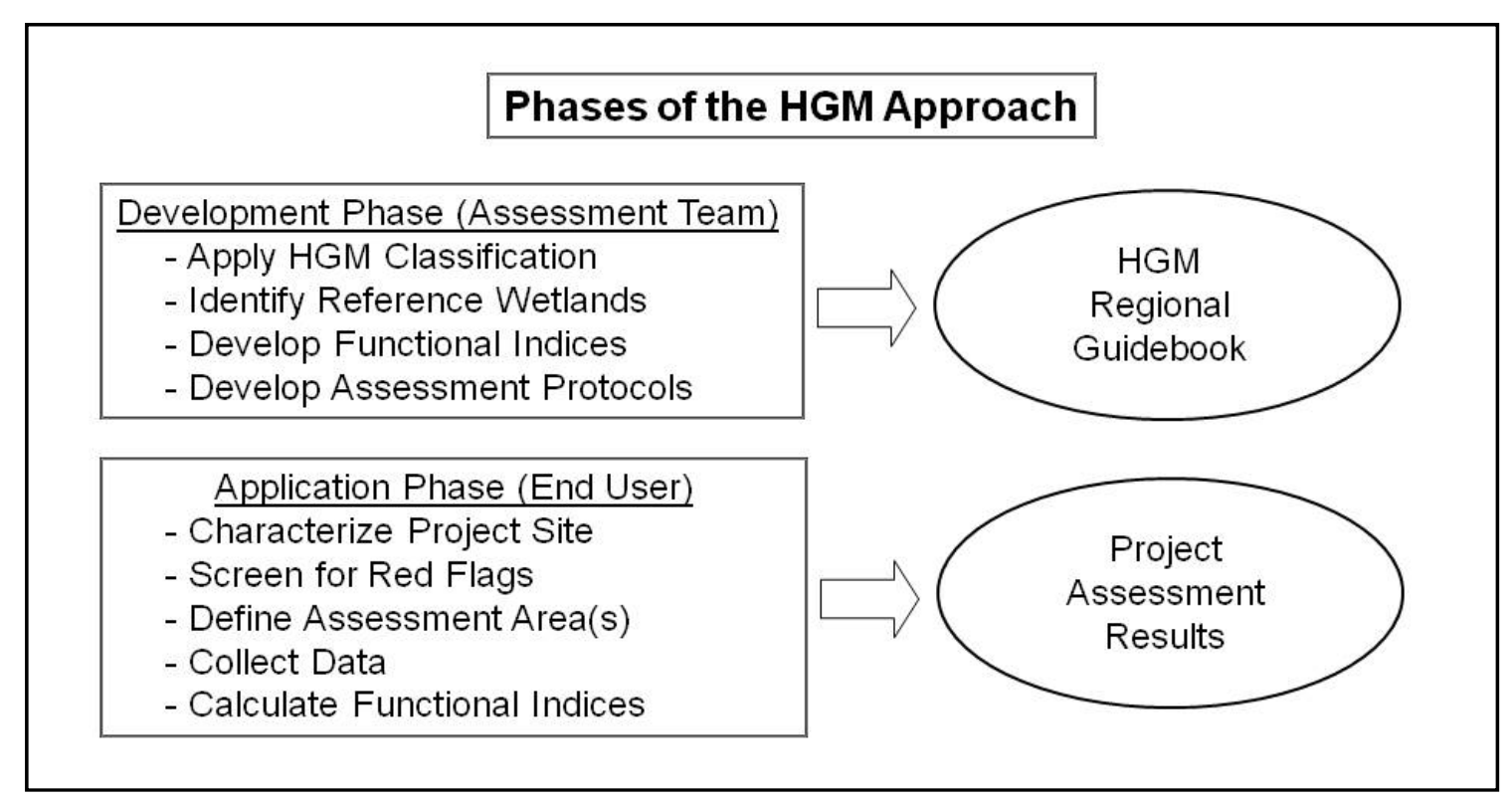

Figure 1. Phases of the HGM Approach.

The development phase of the HGM Approach is carried out by an interdisciplinary team of experts known as the "assessment team." The assessment team begins the development phase by classifying wetlands in 
the geographic area of interest into functional wetland types. One, or more, of these wetland types are selected to develop an assessment procedure. For each wetland type selected, the assessment team identifies a suite of appropriate functions, develops assessment models for deriving functional capacity indices, identifies and collects data from reference wetlands to test and calibrate the indices, and finally develops assessment protocols for endusers. The product of the development phase is a "guidebook" that characterizes the selected wetland type, identifies the functions performed by the wetland type, describes the characteristics and processes that influence how each function is performed, and prescribes end-user protocols for assessing the capacity of a wetland to perform functions.

The application phase of the HGM approach is carried out by a regulator, resource manager, consultant, or other end user who applies the assessment protocols prescribed in the guidebook to assess wetland functions. The context of the assessment may be regulatory, planning, or management. The purpose of the assessment may be to establish baseline conditions, avoid or minimize impacts, compare project alternatives, assess changes in wetland function resulting from project impacts, determine compensatory mitigation requirements, or determine restoration success. In summary, the HGM Approach is best described as a formal process for developing procedures to assess the functions of a specific wetland type in a specific geographic region, and subsequently applying the procedures to assess wetland function in various project-specific contexts.

The HGM Approach was conceived and initially developed at the U.S. Army Engineer Research and Development Center (ERDC) (Smith 1993, Brinson 1993, Smith et al. 1995). Many other individuals and groups have been actively involved in the subsequent development of the HGM Approach. From the beginning it was anticipated that the ongoing task of developing guidebooks would be carried out by various Federal, state, or local agencies, academia, private consultants, non-profit organizations, or other stakeholders with a vested interest in assessing the functions of specific wetland types in specific geographic regions. This document represents a how-to manual for developing guidebooks with the intent to foster consistency in the content, format, technical defensibility, peer review, and application of guidebooks.

This chapter introduces the HGM Approach, describes the subject matter of each chapter in the document, discusses reasons and consideration for 
developing a guidebook, provides overviews of the 404 Regulatory Program in the context of assessing wetland functions and the development phase of the HGM approach. Each of the following chapters provides guidance on a different aspect of guidebook development. Chapter 2, "Identifying and Characterizing Wetland Subclasses," describes the hydrogeomorphic classification and provides guidelines on how to define, geographically delineate, and characterize a wetland subclass. Chapter 3, "Developing Assessment Models," provides guidelines for selecting and defining wetland functions, selecting assessment variables and variable metrics, and constructing a preliminary assessment model based on the literature, available data, and expert opinion. Chapter 4, "Selecting and Sampling Reference Wetlands," provides guidelines for selecting reference wetlands, and collecting and managing the data from reference wetlands. Chapter 5, "Testing and Calibrating Assessment Variables and Verifying Assessment Models," provides guidelines for testing and calibrating assessment variables, and verifying assessment models. Chapter 6, "Developing the Assessment Protocol," provides guidelines for developing the assessment protocols employed by an end-user to assess wetland functions. Chapter 7 , "Validating Assessment Models," provides guidelines for validating assessment models. Chapter 8, "Applications for the Assessment Procedures," provides guidelines and examples for applying the procedures in the guidebook to the assessment of wetland functions, comparing multiple wetlands of the same subclass, computing present and future potential project impacts, and determining mitigation requirements.

\section{Wetland assessment and the 404 Regulatory Program}

The objective of the Clean Water Act (33 U.S.C 1251 et seq.) is to "restore and maintain the physical, chemical, and biological integrity of the Nation's waters." Section 404 of the Clean Water Act (33 U.S.C. 1344) directs the U.S. Army Corps of Engineers (USACE), in cooperation with other agencies, to administer a regulatory program for permitting the discharge of dredged and fill material into "waters of the United States," which include wetlands and other special aquatic sites. The 404 Regulatory Program regulations and guidelines are promulgated in the USACE Regulatory Program Regulations (33 CFR Sections 320-332) and the EPA 404(b)(1) Guidelines (40 CFR Section 230). These regulations and guidelines are subject to amendment and interpretation through Memoranda of Agreement, Regulatory Guidance Letters, and the courts. These documents can be accessed at: http://www.usace.army.mil/CECW/Pages/reg_materials.aspx. 
Applications for a permit to discharge dredged or fill materials undergo a "public interest review" to determine the individual and cumulative effects of a proposed project on wetlands, fish and wildlife, water quality, historical, cultural, scenic, and recreational values, property ownership, and various other factors related to the public interest. The permit review, "...requires a careful weighing of all those factors which may become relevant in each particular case. The benefits which can reasonably be expected to accrue from the proposal must be balanced against its reasonably foreseeable detriments" (33 CFR 320.4 (a)(1)). This report focuses primarily on the effect of a proposed project on wetlands.

An integral part of the permit review is ensuring that any authorized discharge complies with the USEPA 404(b)(1) Guidelines. This requires that the permit review follow a sequence of steps, which include:

1. Evaluate practicable alternatives (40 CFR 230.10 (a))

2. Consider other environmental standards (40 CFR 230.10 (b))

3. Assess significant degradation to waters of the United States (40 CFR $230.10(\mathrm{c}))$

4. Minimize impacts of the discharge (40 CFR 230.10 (d))

One of a number of factual determinations that must be made as part of assessing significant degradation to waters of the United States (Step 3 in the permit review sequence described above) is to..."Determine the nature and degree of effect that the proposed discharge will have both individually and cumulatively, on the structure and function of the aquatic ecosystem and organisms" (40 CFR 230.11 (e)). Simply put, this means that compliance with the 404(b)(1) Guidelines requires determining whether or not the proposed project will have a significant impact on wetland functions. Wetland functions are defined as the normal activities or actions that take place in wetland ecosystems, or simply the things that wetlands do (Smith et al. 1995). In addition, the assessment of wetland functions may be appropriate in other assessment contexts that occur during permit review. These include the evaluation of practicable alternatives (Step 1 in the sequence above), minimizing impacts (Step 4 in the sequence above), establishing compensatory mitigation requirements, and establishing performance standards for mitigation success. "Mitigation is an important aspect of the review and balancing process on many Department of the Army permit applications. Consideration of mitigation will occur throughout the permit application review process and includes avoiding, 
minimizing, rectifying, reducing, or compensating for resource losses. Losses will be avoided to the extent practicable" (33 CFR 320.4 (r)(1)).

USACE Regulatory Headquarters estimates that between 2005 and 2009, the average number of 404 Program permit applications received each year was approximately 73,000 (2010 USACE Regulatory Headquarters). There are two categories of permits for the discharge of dredge or fill material. The first category, identified as "general permits," includes Nationwide, Regional, and Programmatic permits, which are designed to permit discharges resulting from common activities with minimal or minor impacts (e.g., road crossings, piers, ditch maintenance). The second category, identified as "standard" permits," includes Individual permits and Letters of Permission. Individual permits are designed to permit discharges resulting from uncommon activities and large, complex projects with the potential for greater impacts. Letters of Permission are designed for permitting uncommon activities with minimal or minor impacts that do not qualify under a general permit.

Of the 73,00o permit applications received annually, approximately $92.5 \%$ $(67,500)$ were general permits, and approximately $7.5 \%$ (5,500) were standard permits. Of all the permit applications received annually, approximately $75 \%(54,750)$ were general permits that did not require compensatory mitigation. General permits not requiring compensatory mitigation by definition comply with the 404 (b)(1) Guidelines because they result in minimal individual and cumulative impacts, and consequently do not require an assessment of wetland functions. The remaining $25 \%(18,250)$ of all permit applications received annually were permits that did result in unavoidable impacts and therefore required compensatory mitigation, and presumably, an assessment of wetland functions. Of these permits, approximately $83 \%(15,150)$ were general permits with permit conditions requiring compensatory mitigation, and approximately $17 \%$ (3100) were individual permits requiring compensatory mitigation as determined by a public notice and full public interest review.

Despite the fact that more than 18,000 permit applications require an assessment of wetland functions each year, the USACE Regulatory Program Regulations and the USEPA 404(b)(1) Guidelines provide surprisingly little guidance on how to assess wetland functions, or how to determine at what point impacts to wetland functions become "significant." In general, the determination of whether a project will result in a significant impact to 
wetland functions is made subjectively, on a permit-by-permit basis, based on the permit reviewer's knowledge and experience with the 404 Program, wetland ecology, the specifics of the proposed project, and the information supplied by consulting agencies (e.g., USEPA, USFWS, NMFS). While there is no specific guidance on how to assess wetland functions in the permit review assessment contexts related to comparing alternatives, avoiding or minimizing impacts, or determining impacts of a project on wetland functions, specific guidance is provided for determining how much compensatory mitigation is required. This guidance stipulates that wetland functions should be assessed using "appropriate functional or condition assessment methods or other suitable metrics" when available and practicable (33 CFR 332.3 (f)(1)). It further stipulates that if, “...functional or condition assessment or other suitable metrics is not used, a minimum oneto-one acreage or linear foot compensation ratio must be used" (33 CFR 332.3 (f)(1)), and that a mitigation ratio greater than one-to-one may be required under some circumstances (33 CFR 332.3 (f)(2)). It also stipulates that in the case of mitigation banks or in-lieu fee projects, "Where practicable, an appropriate assessment method (e.g., hydrogeomorphic approach to wetland functional assessment, index of biological integrity) or other suitable metric must be used to assess and describe the aquatic resource types that will be restored, established, enhanced and/or preserved by the mitigation bank or in-lieu fee project." (33 CFR 332.8 (o)(2)). It remains unclear how stipulating a mitigation ratio as the default requirement for compensatory mitigation satisfies the requirement to replace the loss of wetland functions resulting from project impacts when no assessment of the loss of wetland functions due to project impacts is actually conducted.

Because there is no clear, objective guidance on how to assess wetland functions in many permit review assessment contexts, it is not surprising that the procedures used during permit review vary considerably in terms of procedural format, documentation, and technical defensibility. In practice, assessment procedures range from the application of a simple ratio, to undocumented, subjective "best professional judgment," to documented, objective procedures developed for specific wetland types in specific geographic regions (USACE 2010). To some extent, the variety of assessment procedures acknowledges that the level of effort associated with permit review may vary depending on the level of impact associated with a project (40 CFR 230.6 (b)), or other factors such as the type of wetland, size and complexity of the project, or assessment context. As a consequence, 
there is some correlation between the level of effort associated with the permit review and the permit category. For example, the effort that goes into the review of a Nationwide permit application with 0.1 acre of unavoidable impacts is normally considerably less than the effort expended to review an Individual permit application with 10 acres of unavoidable impact.

\section{Programmatic and technical criteria}

Assessment procedures developed using the HGM Approach are designed primarily, although not exclusively, to assess wetland functions in the context of 404 permit application review. In order to achieve the widest possible use in this context, assessment procedures were designed to be:

1. Applicable to all wetlands within the geographic extent of USACE regulatory jurisdiction.

2. Compatible with the time and resources normally available to assess wetland functions during 404 permit review.

3. Documented, technically defensible, and capable of assessing wetland functions at a level of precision and accuracy that is appropriate to the assessment of project impacts or restoration efforts.

Developing assessment procedures that satisfy these criteria is a significant challenge (Smith et al. 1995). The HGM Approach addresses the challenge by employing a unique combination of techniques and strategies that include a separate development phase and application phase, a hydrogeomorphic classification to identify classes of functionally similar wetlands, reference wetlands to establish a range of natural variation and standards of comparison for each function, and others. Each of these techniques and strategies are discussed below in terms of the programmatic and technical criteria identified above.

\section{Criteria 1: Applicable to all wetland types}

The HGM Approach can be used to develop a procedure to assess any wetland type that occurs within the geographic extent of USACE regulatory jurisdiction. To date, the HGM Approach has been used to develop guidebooks for a variety of wetland types in a variety of locations in the United States. A list of guidebooks published by ERDC can be found at: http://el.erdc.usace.army.mil/wetlands/guidebooks.cfm. 


\section{Criteria 2: Compatible with time and resources available for 404 Permit review}

The HGM Approach achieves compatibility with the time and resources that are normally available to assess wetland functions during permit review by developing assessment procedures that are both "rapid" and adaptable to the various assessment contexts that can occur during permit review. As indicated above, the contexts in which the assessment of wetland functions is appropriate include the determination of whether or not the proposed project will have a significant impact on wetland functions, the evaluation of practicable alternatives, minimization of project impacts, and establishing compensatory mitigation requirements and performance standards for mitigation success.

The phrase rapid assessment and the term wetland have been linked since at least 1983 when Adamus (1983) characterized his "Method for Wetland Functional Assessment” as a “...rapid assessment procedure for screening functional values of wetlands." Nearly 30 years later, the discussion on what constitutes a rapid wetland assessment continues (Kleindl et al. 2010). Based on the literature (Kusler and Riexinger 1986; Ainslie 1994; Fennessy et al. 2004, 2007; Kleindl et al. 2010) and discussions with numerous regulators over many years, there appears to be an informal consensus that a "rapid" assessment procedure is one that under normal circumstances can be completed in one day. This is consistent with the definition of a rapid assessment procedure suggested by Fennessy et al. (2004, 2007), and with the Level 2 - Rapid Wetland Assessment category suggested in the Technical Framework for Wetland Monitoring and Assessment (USEPA 2002b). Individuals experienced with the HGM Approach report that, under normal circumstances, the protocols for assessing wetland functions prescribed in guidebooks can be completed in one day or less, including preparation time, travel time, field data collection, and analysis (Whigham et al. 2007, Kleindl et al. 2010, Berkowitz et al. 2011).

Several different strategies make it possible to develop a rapid assessment procedure using the HGM Approach. One is the separation of the development and application phases (Figure 1). The development phase provides the assessment team with the time and resources necessary to collect, analyze, synthesize, and compile new and existing information into a guidebook. During the application phase, end-users are able to leverage the efforts expended during the development phase, through the use of the assessment protocols in the guidebook, to rapidly assess wetland functions. 
In addition, the guidebook provides end-users with a succinct, current synthesis of the wetland type, including wetland classification and characterization, a description of the important functions, and a description of the important factors that influence the capacity of the wetland type to perform functions.

Another strategy that makes it possible to develop rapid assessment procedures is the use of hydrogeomorphic classification. Wetland ecosystems exhibit a wide natural range of variation in terms of their physical, chemical, and biological characteristics and processes (Mitsch and Gosselink 2000, Cowardin 1979). Consider the range of variation exhibited in vernal pools in Mediterranean climates with precipitation-driven wet-dry cycles and endemic, herbaceous vegetation, southeastern bottomland hardwood forests linked to seasonal overbank or backwater flooding, and salt marshes with extensive stands of monotypic vegetation and tidal hydrologic regimes. Wetland functions, the normal activities or actions that take place in wetland ecosystems, or simply the things that wetlands do (Smith et al. 1995), reflect the characteristics and process of the particular wetland and its surrounding landscape. Variations in wetland characteristics and processes dictate which functions a wetland performs, how those functions are performed, and to what degree or magnitude those functions are performed. Because of this "functional variation," not all wetlands perform the same function in the same way, or to the same degree or magnitude, if at all. The need to account for functional variation is recognized in the 404 Program Regulations, which state, "Where appropriate, district engineers shall account for characteristics of aquatic resource types, functions and services when determining performance standards and monitoring requirements for compensatory mitigation projects (33 CFR 332.1 (e)). For example, few would attribute a sediment removal function to bogs with precipitation as the sole source of water, much less claim that they performed the function to the same degree or magnitude as bottomland hardwood forests on river floodplains in the Southeast.

The range of natural variation in function that wetland ecosystems exhibit presents a challenge to the assessment of wetland functions. The essence of the challenge is how to develop assessment procedures that are both rapid and capable of capturing the variation and complexity exhibited by wetland ecosystems. There are at least two possible approaches, one that generalizes and one that specializes. The generic approach begins with the assumption that all wetlands perform a similar suite of functions in the 
same way, and rely on generic, one-size-fits-all, assessment procedures that emphasize the similarities of wetland ecosystems. Assessment procedures based on a generic approach abound, and Lonard (1981), USEPA (1984), World Wildlife Fund (1992), and Bartoldus (1999) describe more than 40 assessment procedures developed over the last 30 years, many of which employ a generic approach (e.g., Amman and Stone 1991; Adamus et al. 1987).

Generic assessment procedures can be useful in situations where a less accurate, categorical assessment (e.g., high, moderate, or low) provides adequate information to satisfy project objectives. For example, assessments designed to screen or prioritize a large number of wetland sites across a large geographic area, or similar scenarios that fall under the USEPA Level 1 Assessment category (USEPA 2002). However, generic assessment procedures are not well-suited for 404 permit application review for several reasons. First, some functions in a generic suite of functions may not be relevant to the particular wetland type under consideration, or vice versa, a function that is important to a particular wetland type may not be included in a generic suite of functions. Second, it is often difficult with generic assessment procedures to achieve a level of accuracy and precision that makes it possible to detect when a significant change in wetland function has occurred as a result of project impacts. Finally, in order to measure the impacts of a project to wetland functions, mitigation requirements, and mitigation/restoration success, it is necessary for an assessment procedure to provide a quantitative measure of wetland function.

The alternative to the generic approach is to simplify the assessment procedure by reducing the functional variation that is considered during an assessment. As described in Chapter 2, the hydrogeomorphic classification was specifically designed to identify functionally similar groups of wetlands within a prescribed geographic region (Brinson 1993). Reducing functional variation narrows the focus of attention to those functions that are performed by a specific group of wetlands, and the characteristics and processes that influence those functions. This simplifies the development of assessment procedures and makes it possible for assessment procedures to be both rapid, and capable of measuring changes in wetland functions at a level of resolution that is appropriate for assessing project impacts or restoration success.

Another way in which the HGM Approach achieves compatibility with the 404 program or project objectives is through the development of 
assessment procedures that are adaptable to the various assessment contexts that can occur during the development of project objectives. Guidebooks prescribe an assessment protocol that can be applied rapidly in the sense that it can be completed in one day or less. However, the potential also exists to streamline the assessment protocol even further for application in situations where standardized assessment scenarios occur repeatedly, as is the case with some general permits. Currently, many districts purport to assess wetland functions by calculating compensatory mitigation requirements for unavoidable impacts based on mitigation ratios that are linked to wetland type or other weighting factors that give little or no consideration to on-the-ground conditions of either the mitigation or impact site (USACE, Charleston District 2002). Table 1 illustrates a different approach that pre-calculates mitigation ratios for common impact and mitigation scenarios based on the assessment models, reference data, and variable curves prescribed in the guidebook. The advantage of this approach is that the mitigation ratios are based on a documented and technically defensible set of assessment models prescribed in the guidebook rather than subjective, technically indefensible, or undocumented criteria or rationale.

Table 1. Mitigation ratios and potential FCl for Low Gradient Riverine Backwater Wetlands in the Southeast.

\begin{tabular}{|l|l|l|l|l|l|l|}
\hline & & & \multicolumn{3}{|c|}{ Impact Scenario / Mitigation Ratios (below) } \\
\cline { 4 - 7 } & $\begin{array}{l}\text { Potential } \mathrm{FCl} \\
\text { Restoration Scenario }\end{array}$ & $\begin{array}{l}\text { Mature } \\
\text { Increase in Restored }\end{array}$ & $\begin{array}{l}\text { Forested } \\
\text { Wetland } \\
\text { FCl }\end{array}$ & $\begin{array}{l}\text { High-graded } \\
\text { Forested } \\
\text { Wetland } \\
(\mathrm{FCl}=1.00)\end{array}$ & $\begin{array}{l}\text { Scrub/ } \\
\text { Clearcut } \\
\text { Wetland } \\
(\mathrm{FCl}=0.80)\end{array}$ & $\begin{array}{l}\text { Farmed } \\
\text { Wetland } \\
(\mathrm{FCl}=0.07)\end{array}$ \\
\hline $\begin{array}{l}\text { Reforest and Restore } \\
\text { Microtopographic } \\
\text { Relief to Farmed } \\
\text { Wetland }\end{array}$ & $\mathrm{FCl}=0.70$ & $\mathrm{FCl}=0.50$ & $2.00: 1$ & $1.61: 1$ & $0.93: 1$ & $0.14: 1$ \\
\hline $\begin{array}{l}\text { Restore Hydrology and } \\
\text { Reforest Drained and } \\
\text { Farmed Former } \\
\text { Wetland }\end{array}$ & $\mathrm{FCl}=0.70$ & $\mathrm{FCl}=0.56$ & $1.77: 1$ & $1.43: 1$ & $0.82: 1$ & $0.12: 1$ \\
\hline $\begin{array}{l}\text { Restore Hydrology to } \\
\text { Drained Mature } \\
\text { Forested Wetland }\end{array}$ & $\mathrm{FCl}=0.99$ & $\mathrm{FCl}=0.24$ & $4.23: 1$ & $3.41: 1$ & $1.96: 1$ & $0.29: 1$ \\
\hline $\begin{array}{l}\text { Restore Hydrology to } \\
\text { Drained and Degraded } \\
\text { Forested Wetland }\end{array}$ & $\mathrm{FCl}=0.92$ & $\mathrm{FCl}=0.41$ & $2.41: 1$ & $1.94: 1$ & $1.11: 1$ & $0.16: 1$ \\
\hline $\begin{array}{l}\text { Modify Non- Wetland } \\
\text { to Establish Wetland } \\
\text { Hydrology and Woody } \\
\text { Vegetation }\end{array}$ & $\mathrm{FCl}=0.64$ & $\mathrm{FCl}=0.49$ & $2.03: 1$ & $1.63: 1$ & $0.94: 1$ & $0.14: 1$ \\
\hline
\end{tabular}




\section{Criteria 3: Documented, technically defensible, and compatible}

Assessment procedures developed using the HGM Approach are selfdocumenting in the sense that guidebooks synthesize both the information collected and analyzed during the development phase as well as the protocols that end-users employ in assessing wetland functions. Assessment procedures are technically defensible because they undergo iterative review, verification, and field testing by the assessment team during the development phase as well as a formal, external peer review prior to publication.

\section{Overview of the development phase}

The development phase of the HGM Approach follows the sequence of tasks prescribed in the National Action Plan (Federal Register, June 20, 1997, 62(119), pp. 33607-33620, Table 2-1). While there is a logical progression to the sequence of tasks, they are not mutually exclusive, and consequently the development of a guidebook is an iterative process that often requires a re-examination or revision of data or information from a previous task based on new data or information. For example, the wetland types identified during Task 2 based on the experience of the team members may be revised based on information following the collection of reference wetland data during Task 5 .

The eight tasks necessary to develop a guidebook are described briefly below, and discussed in greater detail in subsequent chapters of this document.

\section{Task 1: Organize assessment team}

\section{Objective}

The objective of this task is to select and organize the interdisciplinary team of technical experts who can provide useful information and review for each phase of the development of the guidebook.

Approach

Guidebooks are developed by a group of technical experts known as the assessment team, or A-Team. The team typically consists of six or more individuals who, as a group, possess a broad interdisciplinary understanding of wetlands in the geographic area of interest. Assessment team members can be affiliated with Federal, state, or local agencies, nongovernmental organizations, other stakeholders, private consultants, or 
academia. The role of the assessment team is to develop the guidebooks in a manner that is consistent with the tasks outlined in Table 2 (National Action Plan (Federal Register, June 20, 1997, 62(119), pp. 33607-33620).

Table 2. Tasks in developing HGM Guidebooks.

Task 1: Organize the Assessment Team

A. Identify team members

B. Train team in the HGM Approach

Task 2: Select and Characterize Wetland Subclass

A. Identify and prioritize wetland subclasses

B. Select wetland subclass

C. Define reference domain

D. Characterize wetland subclass

Task 3: Select Functions, Variables, Metrics and Develop Conceptual Assessment Models

A. Select and define wetland functions for wetland subclass

B. Review existing assessment models for selected functions

C. Identify potential assessment variables and metrics

D. Develop conceptual relationship between variables and functional capacity

E. Construct conceptual assessment models for deriving Functional Capacity Index ( $\mathrm{FCl}$ )

F. Complete Pre-calibrated Draft Guidebook (PDG)

Task 4: Conduct Peer Review of Pre-calibrated Draft Guidebook

A. Distribute PDG to peer reviewers

B. Conduct interdisciplinary, interagency workshop of PDG

C. Revise PDG to reflect peer review recommendations

D. Distribute revised PDG to peer reviewers for comment

E. Incorporate final comments from peer reviewers on revisions into the PDG

Task 5: Select and Sample Reference Wetlands
A. Identify reference wetland field sites
B. Collect data from reference wetland field sites
C. Manage and prepare reference wetland data for analysis

Task 6: Test and Calibrate Assessment Variables and Models

A. Test and calibrate assessment variables using reference wetland data

B. Verify and validate (optional) assessment models

C. Field test assessment models for accuracy, repeatability, and user-friendliness

D. Revise PDG based on calibration, verification, validation (optional), and field test results into a Calibrated Draft Guidebook (CDG)

Task 7: Conduct Peer Review and Field Tests of Calibrated Draft Guidebook

A. Distribute $C D G$ to peer reviewers

B. Field test CDG

C. Revise CDG to reflect peer review and field test recommendations

D. Distribute CDG to peer reviewers for final comment on revisions

E. Incorporate peer reviewers' final comments on revisions

F. Publish Operational Draft Guidebook (ODG)

Task 8: Technology Transfer

A. Train end users in the use of the ODG

B. Provide continuing support and technical assistance to the ODG end-user 
The time required to develop a guidebook normally ranges from 1-3 years. In practice, the actual time required will depend on a variety of factors, including the ability of the assessment team members to commit time, the number of wetland types and size of the geographic area under consideration, whether the work is done by the assessment team or with the assistance of contractors, and the skill of the team administrator to organize, schedule milestones, delegate work, schedule meetings, coordinate peer review, and in general keep the process moving along. The assessment team is normally administered by an individual from the Corps of Engineers, but that responsibility can be designated to a non-Corps team member. The role of administrators is crucial to the success of the guidebook, and the demand on their time is substantial. The time demand on other team members is often considerably less. Contracting specific tasks can reduce the time required to develop a guidebook, but will also increase the cost.

\section{Products}

The first meeting of the assessment team should include training in the HGM Approach and the development of guidebooks, the development of a proposed timeline for completing the sequence of tasks identified in Table 2, and the delegation of specific responsibilities to individual team members. Responsibilities should be delegated based on tasks, functions, or another scheme that maximizes the time, effort, and expertise of each team member. The assessment team should schedule meetings to correspond with the initiation and completion of major tasks. Discussion items and key decisions at each meeting should be documented in meeting minutes. This documentation will be invaluable in preparing the guidebook.

\section{Task 2: Classify and characterize wetland subclass}

\section{Objective}

The objective of this task is to identify the geographic area of interest, classify the different types of wetlands, or "wetland subclasses" in the terminology of the HGM Approach (see Chapter 2, "Classify and Characterize Wetland Subclasses"), select the wetland subclass for which a guidebook will be developed, and identify, review, and acquire existing literature, data, and other information relevant to the selected wetland subclass. 
Approach

The assessment team begins this task by classifying wetlands in the geographic area of interest into wetland subclasses. Chapter 2, "Classify and Characterize Wetland Subclasses," provides detailed information on a variety of sources that will be helpful during this process. In addition, in many parts of the country, existing wetland classifications can serve as a starting point for identifying wetland subclasses (Stewart and Kantrud 1971, Golet and Larson 1974, Wharton 1978, Keeler-Wolf et al. 1998).

Next, the assessment team should select the wetland subclass for which a guidebook will be developed. This selection will often be based on the predetermined needs of the regulatory agencies or developmental pressures on particular wetland types in the region. The assessment team should then establish preliminary boundaries of the geographic area from which reference wetlands will be selected and sampled. Finally, the assessment team begins a search for and review of literature and data relevant to the wetland subclass selected.

\section{Products}

A narrative description should be developed to classify wetland subclasses in the geographic area of interest. A dichotomous key for identifying wetland subclasses can be helpful when a guidebook for more than one wetland subclass is being developed. The narrative characterization can include a discussion of climate, geomorphic setting, water sources, hydrodynamics, soils, vegetation, wildlife, and predominant types of natural and anthropogenic disturbances. The characterization should be supported by literature where possible. Throughout the development phase, the assessment team should search for, acquire, maintain, update, and make available, to all team members and peer reviewers, information relevant to the development of a guidebook for the wetland subclass.

\section{Task 3: Develop conceptual assessment models}

\section{Objective}

The objective of this task is to identify and define a suite of wetland functions for a wetland subclass, and develop a conceptual assessment model for each function. 
Approach

The development of conceptual assessment models begins with the identification, definition, and description of a suite of wetland functions for a wetland subclass. An important aspect of this process is designating the quantitative, independent measure of the function that can potentially be used to validate the assessment model (see Chapter 7, "Validate Assessment Models"). The selection of wetland functions can be initiated and facilitated by the review of previously published guidebooks developed as part of the HGM Approach. These documents define and describe a suite of wetland functions selected for specific wetland subclasses, outline the criteria used in the selection process, explain why a function was selected, and provide references to the sources of information used in the selection process. These sources should be supplemented with other sources of information on wetlands and wetland functions.

Once wetland functions have been identified, a conceptual assessment model is developed for each function. Assessment models consist of a mathematical equation that prescribes the way in which assessment variables are combined to derive a Functional Capacity Index (FCI). The FCI is a relative measure of the capacity of a wetland to perform a function. Assessment variables represent attributes, characteristics, and processes of the wetland, and the surrounding landscape, that influence how a wetland performs the function. Assessment variables are measured using a qualitative or quantitative variable metric with a defined relationship to functional capacity.

The initial development of conceptual assessment models is based on the literature, existing data, and the knowledge and experience of the assessment team. The assessment team should contribute to identifying and defining assessment variables, variable metrics for measuring assessment variables, the relationship between variable metrics and functional capacity, and the mathematical equation used to combine variables and derive that FCI. The initial assessment variables, variable metrics, and relationships in the conceptual assessment model will subsequently be tested, calibrated, and potentially revised (see Chapter 5, "Calibrate, Verify, and Field-Test Assessment Models") following the collection and analysis of reference wetland data (see Chapter 4, "Reference Wetlands and Reference Wetland Data." Once variables are selected, an assessment protocol that the end-user will employ to assess wetland function in the context of a specific project must be developed. The assessment protocol should provide a step-by-step 
procedure for bounding the assessment area, laying out sample locations, collecting variable metric data, completing field-sheets, and calculating the FCI for each function.

\section{Products}

The product of this task is a Pre-calibrated Draft Guidebook (PDG). With the exception of potential revisions of assessment models based on testing and calibration using reference wetland data, the PDG should include all of the components found in a complete guidebook outlined in Table 3.

\section{Task 4: Peer review of pre-calibrated draft guidebook}

\section{Objective}

The objective of this task is to identify a suitable group of peer reviewers, submit the PDG for peer review, conduct a workshop attended by peer reviewers, and revise the PDG based on peer reviewer comments and workshop results.

\section{Approach}

After completing the PDG, a suitable group of peer reviewers should be identified. Peer reviewers should have extensive experience and knowledge with both the wetland subclass under consideration and the HGM Approach. Peer reviewers can include individuals representing both the end-user community and technical experts that include a cross section of Federal and state agencies, academia, non-governmental groups, and the private sector. Peer reviewers should have either substantial regulatory experience or technical experience in hydrology, biogeochemistry, plant ecology, wildlife ecology, or other disciplines relevant to the wetland subclass under consideration.

The peer review process is initiated with a review of the PDG. Upon completing the review, peer reviewers should provide comments and recommendations to the assessment team for review, compilation, and summarization. Peer reviewers should also provide a list of literature that is relevant to the wetland subclass as well as any suggestions for potential reference wetland sites. 
Table 3. Guidebook outline.

Chapter 1: Introduction (A generic version of this chapter is available from the Wetlands and Coastal Ecology Branch at ERDC. It requires only minor modification in order to be used in a specific guidebook.)

- Background

- Objectives

- Purpose of the Guidebook

Chapter 2: Overview of the HGM Approach (This chapter is prepared by the ERDC Environmental Laboratory and requires only minor tailoring for each specific guidebook.)

- Description of Development and Application Phase of the HGM Approach

- Hydrogeomorphic Classification

- Assessment Models

- Conceptual Assessment Model

- Testing and Calibrating Assessment Variables and Verifying Assessment Models

- Assessment Protocol

Chapter 3: Characterization of the Subclass

- Define Reference Domain

- Define Potential Geographic Extent of the Subclass

- Characteristics of the Subclass

- Climate

- Geomorphic Setting

- Hydrodynamics

- Soils

- Vegetation

- Wildlife

- Anthropogenic Disturbances

\section{Chapter 4: Wetland Functions and Assessment Models}

\section{- Wetland Functions Overview}

- Function 1 (Repeat Sequence for other Functions)

- Definition

- Rationale for Selection of Function (importance to the Wetland Subclass)

- Discussion of attributes, characteristics, and processes that influence the function and how they interact to influence the function

- Assessment Model Variables

- Variable 1 (Repeat Sequence for other Variables)

- Definition

- Rationale (why include this variable?)

- Measurement

- Calibration

- Transformation of Field Measure to Subindex to Include Range of Reference Data

- Functional Capacity Index (FCl)

- Aggregation Equation

- Rationale for Relationship Between Variables (i.e., weighting, combinations, etc.) 


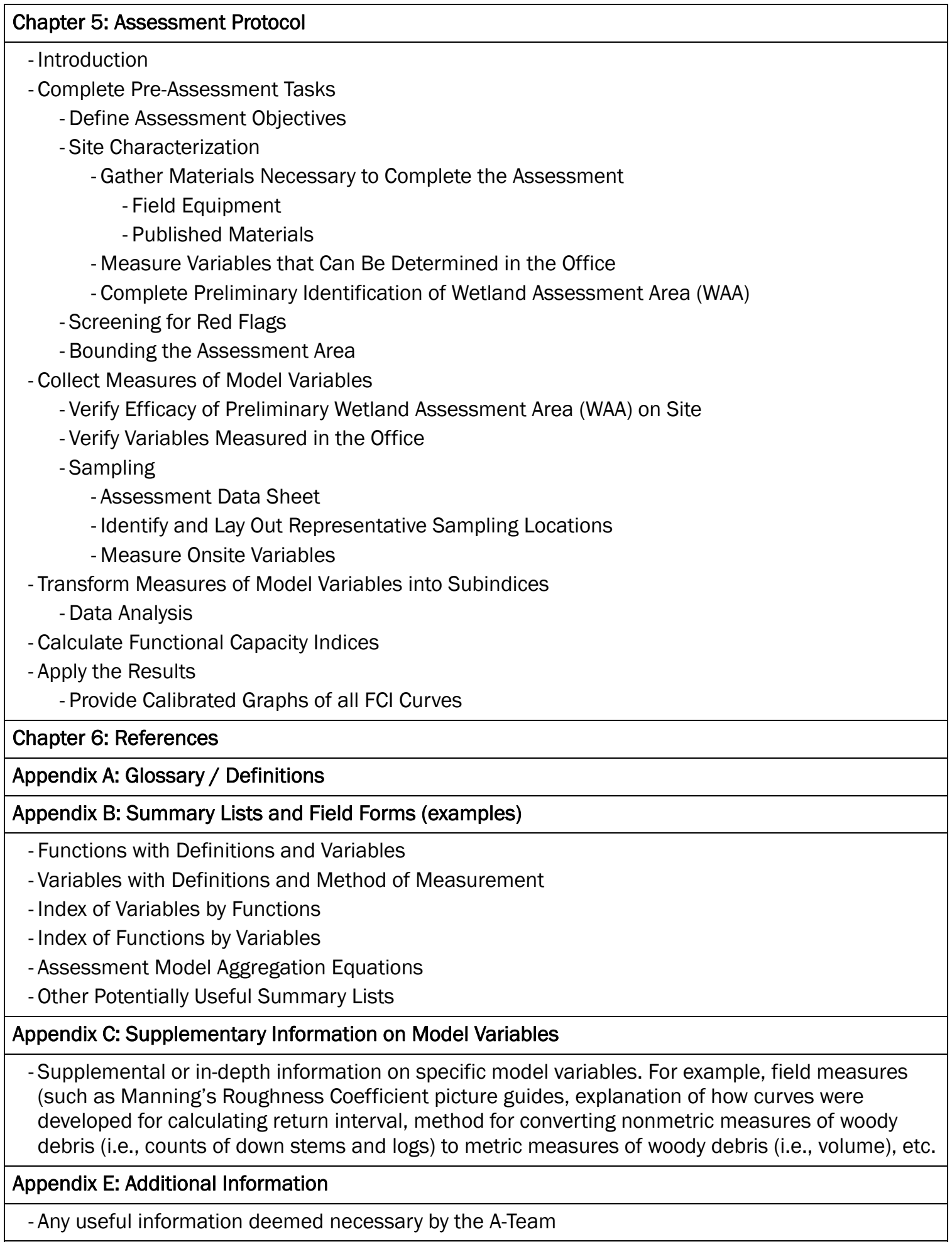

In some cases it may be beneficial to convene a workshop that brings all peer reviewers together. The workshop might begin with an overview of the HGM Approach and the PDG, and a summary of key comments and recommendations resulting from the individual peer reviews. If the PDG is 
large, it can be helpful to break into workgroups for different disciplines. Workgroups should contain individuals representing the end-user community and technical experts as well as at least one member of the assessment team. Each workgroup should have a facilitator, and a recorder. Those functions with a significant hydrology component should be reviewed in the hydrology workgroup, those related to biogeochemistry in the biogeochemistry workgroup, and so on. The workgroup should review all aspects of the guidebook related to the assigned functions, including definitions, rationale for including the function, assessment variables, variable metrics, the relationship between variable metrics, and the mathematical equation used to derive the FCI. Workgroup recommendations may include the deletion, addition, or modification to functions, assessment models, assessment variables, or variable metrics. Recommended changes may be accompanied with a rationale for the deletion, addition, or modification, and a full description of the proposed changes.

At appropriate times during the workshop, the workgroups should reconvene and present progress reports and recommendations being considered for the benefit of other workgroups. Recommendations suggested by one workgroup, but relevant in another, should also be discussed and, if appropriate, reassigned to the appropriate workgroup. To conclude the workshop, all workgroups should reconvene and present a final summary of their recommendations. After the workshop, the recommendations of each workgroup should be reviewed, the PDG should be modified as appropriate, and the revised PDG should be sent back to reviewers for final review and comment.

\section{Products}

The PDG will serve as a working document during the data collection phase. As data are collected from reference sites or reference wetlands, the PDG will undergo changes based on the knowledge gained during the data collection analysis phases of guidebook development.

\section{Task 5: Select and sample reference wetlands}

\section{Objective}

The objective of this task is to identify a pool of potential reference wetland sites, select reference sites from the pool, collect data from selected reference wetlands, and manage the flow of reference wetland 
data from field data forms to digital formats in preparation for data analysis.

\section{Approach}

Reference wetlands are specific wetland sites within a geographic area of interest, or "reference domain" that represent the full range of conditions exhibited by wetlands in the wetland class. Reference wetlands serve several purposes in the HGM Approach. They provide a physical representation of wetlands in the subclass that can be observed and sampled repeatedly, establish the range of conditions exhibited by variable metrics (making it possible to calibrate model variables and functional capacity indices), and serve as a template for developing design specifications for wetland restoration (i.e., reference standard conditions). Reference wetlands are not undisturbed, pristine wetlands as outlined in other documents.

The first step in selecting reference wetlands is to conduct an inventory of the wetlands belonging to the wetland subclass in the reference domain. Due to their local knowledge of the wetlands subclasses, this is often one of the most valuable roles of the assessment team. Once the inventory is complete, the next step is to begin to conduct field reconnaissance to screen potential candidate reference wetland sites. The objective is to identify sites that represent the full range of conditions that exist in the reference domain from highly altered sites in highly altered landscapes to unaltered sites in unaltered landscapes. For example, in a riparian forested wetland subclass, the reference wetland sites would include undisturbed, highly functional wetlands, moderately disturbed, and heavily impaired wetland sites. The process of inventory and selection of reference wetlands is described in greater detail in Chapter 4, "Reference Wetlands and Reference Wetland Data."

Once reference wetlands have been identified, data must be collected from reference wetlands. This process includes managing and preparing the data for further analysis. This is perhaps the most time-consuming and resourceintensive process in developing the guidebook. It involves developing forms for documenting reference wetlands and assessment variables, developing protocols and field sampling forms for variable metrics, as well as actually collecting and managing reference wetland data. Each of these tasks is described in greater detail in Chapter 4, "Reference Wetlands and Reference Wetland Data." 


\section{Products}

A list of potential reference wetlands should be identified and documented. Information that has proven helpful during the inventory of potential reference wetlands includes: the basis for selecting reference wetlands, GIS data layers for the location of reference wetlands, political boundaries, infrastructure, land uses, soil type, rivers and streams and other information used during the inventory, and selection and documentation of reference wetlands.

Original hardcopies and scanned electronic copies of the reference wetland documentation forms and field sampling forms should be maintained. Databases or spreadsheets containing reference wetland data from field sampling forms should be developed and maintained for further data analysis.

\section{Task 6: Test and calibrate assessment variables and verify assessment models}

Objective

The objective of this task is to test and calibrate assessment variables using reference wetland data, and verify assessment models using sensitivity analysis and field testing.

\section{Approach}

The testing and calibration process includes screening model variables, calibrating model variables using reference wetland data, and verifying and field testing assessment models. These tasks represent relatively simple aspects of testing and calibration. For example, a test of model logic and sensitivity can be accomplished in less than an hour, and the results can be used immediately to guide the revision of an assessment model. On the other hand, validation is a more complex and time-consuming aspect of testing and calibration that can involve years of additional research and data gathering that is far beyond the interests, capabilities, or responsibilities of the assessment team and generally not part of typical guidebook development.

Testing and calibration begin with the screening of variable metrics in order to determine their ability to discriminate between various types and degrees of disturbance across the range of conditions exhibited in reference wet- 
lands. For metrics that discriminate between various types and degrees of disturbance, the relationship between metric values and variable subindices can be calibrated based on an analysis of reference wetland data. The process of testing and calibrating assessment variables is described in greater detail in Chapter 5, "Calibrate, Verify, and Field-Test Assessment Models."

Verification of assessment models is a procedure meant to determine whether model output makes sense, from a logical perspective, and should not be confused with model validation or testing for accuracy. To verify the logic of assessment models, apply the model to real or hypothetical data and evaluate the results in light of experience and understanding of the wetland subclass. The two basic approaches the assessment team should use in verifying the assessment models are sensitivity analysis and applying the model to sample data sets.

Sensitivity analysis is an appraisal of model performance under incremental change in the input variables. Sensitivity analysis helps to verify that the model behaves as intended under both moderate and extreme levels of each variable. An important goal of sensitivity analysis is to identify the variables in the model with the most influence on the FCI, and, conversely, those variables that have little influence on the FCI. Analyzing the sensitivity of an assessment model cannot verify that the model will respond appropriately to field measurements. This can only be done by inputting the actual measures for each variable and examining both the resulting variable subindices and the FCI. Finally, field testing the assessment model is designed to ensure that an end-user can apply the assessment model efficiently and with consistent results. The process of analyzing the sensitivity of the assessment model, applying the assessment model to sample data sets, and field testing is described in greater detail in Chapter 5, "Calibrate, Verify, and Field-Test Assessment Models."

\section{Products}

The product of this task is a Calibrated Draft Guidebook (CDG) that is ready for peer review.

\section{Task 7: Conduct peer review and field testing of calibrated draft guidebook} Objective

The objective of this task is to conduct a peer review of the Calibrated Draft Guidebook (CDG) after assessment variables have been tested and calibrated, and assessment models have been verified. 
Approach

Each peer reviewer conducts an evaluation of the PDG. Upon completing the evaluation, peer reviewers should provide comments and recommendations to the assessment team for review, compilation, and summarization. The assessment team then revises the CDG as appropriate, conducts a field test of the revised CDG across a range of conditions, revises the CDG based on the results of field testing, and then sends the revised CDG to peer reviewers for final review and comment.

\section{Product}

The product of this task is an Operational Draft Guidebook (ODG), which incorporates appropriate comments and recommendations from peer reviewers and the results of field testing.

\section{Task 8: Technology transfer}

\section{Objective}

The objective of this task is to train and provide ongoing support and assistance to end users of the Operational Draft Guidebook (ODG).

\section{Approach}

This task is accomplished by conducting a 1- to 5-day field-oriented training course to potential end users of the ODG. The training course should be led by members of the assessment team and include an introduction to the HGM Approach and discussion of all the functions, assessment variables, variable metrics, assessment models, and assessment protocols from the guidebook. In addition, the course should provide experience in the various potential applications of the assessment procedure.

One approach that has proven to be useful is to divide the class into small teams of three to five individuals to conduct field sampling and data analysis. At the first field site, the team should be given a step-by-step demonstration of the assessment protocols prescribed in the guidebook. This will familiarize the teams with the field forms, layout of sample plots, and procedures for collecting data. Teams then move to another field site where a hypothetical project is planned. Each small team should go through the assessment protocol once to collect data that represent pre-project conditions (i.e., existing) and a second time to collect data that represent 
post-project conditions based on expected project impacts. A comparison of pre-and post-project conditions will provide a measure of potential project impacts (i.e., change in FCI), and a measure of mitigation requirements.

At a third field site, representing a potential mitigation site, teams should complete the assessment protocol to represent pre-project conditions (i.e., existing). Each team should prepare a mitigation plan that proposes specific restoration activities, and then complete the assessment protocol for the conditions that are expected to exist after the proposed restoration activities in the mitigation plan have been implemented. A comparison of pre- and post-restoration FCI will help determine whether the restoration activities proposed in the mitigation plan adequately offset the project impacts identified at the proposed field site. During the last day of the training course, each team will present the results of their field work to discuss and compare findings within the group. 


\section{Classify and Characterize Wetland Subclasses}

\section{Introduction}

The chapter begins with a brief description of the Hydrogeomorphic Classification (Brinson 1993) and the role it plays in reducing the range of natural variation in function that is considered when assessing wetland functions. It provides guidance for the tasks of regionalization, defining wetland subclasses, and identifying the geographical boundaries of the reference domain and general considerations for determining the number of wetland subclasses to include in the guidebook. It also identifies key aspects of characterizing wetland subclasses.

\section{Hydrogeomorphic Classification}

The Hydrogeomorphic Classification (hereafter HGM Classification) is a hierarchical system for classifying wetlands based on three factors that Brinson (1993, 1995) described as "first principles" of wetland function. The first factor is geomorphic setting, or the position of a wetland in the landscape. Wetlands occur in a variety of geomorphic settings, including topographic depressions, hillslopes, floodplains, interfluves, and tidal areas, among others. The second factor is dominant water source, or the primary, proximate source of the water in a wetland. While all freshwater originates as precipitation, it often follows a circuitous path to the wetland proper via the surface of the ground (e.g., overland, channel, or overbank flow), below the ground surface (e.g., interflow, throughflow, or baseflow), or some combination of these pathways (Figure 2). The third factor is hydrodynamics, or the movement of water in a wetland (e.g., horizontal flow and vertical fluctuation) and level of energy associated with the movement of water in a wetland. For example, in most depression wetlands, water fluctuates vertically with a relatively low level of energy, whereas in riverine wetlands water flows unidirectionally downstream with relatively high levels of energy.

As discussed in Chapter 1, wetlands exhibit a wide range of natural variation in terms of their physical, chemical, and biological characteristics and processes (Mitsch and Gosselink 2000, Cowardin 1979). This variation results in a similarly wide range of natural variation in which functions 


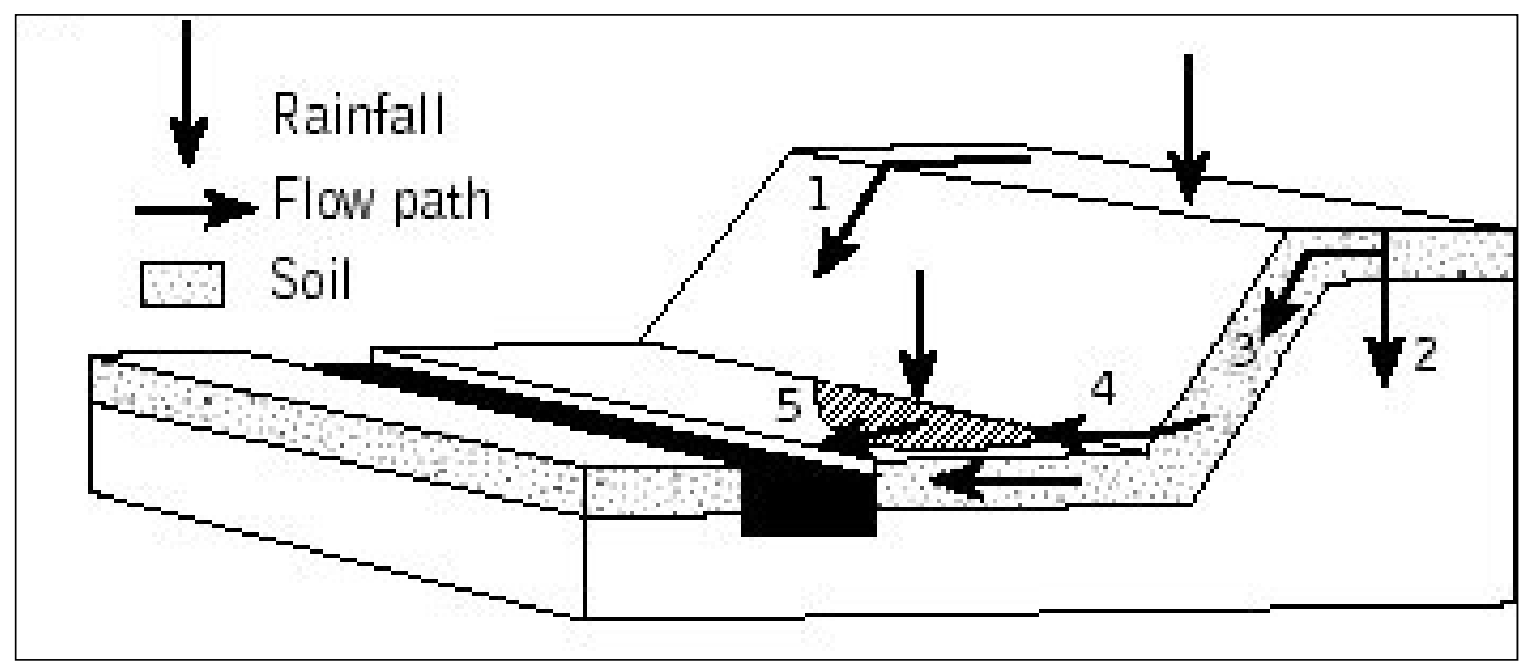

Figure 2. Possible flow paths of rainfall on hill slopes, including (1) infiltration-excess overland flow; (2) groundwater recharge; (3) throughflow; (4) return flow; and (5) saturation overland flow.

different types of wetlands perform, and the degree or magnitude to which those functions are performed. In HGM Approach, the role of the HGM classification is to reduce the range of natural variation by identifying classes of wetlands that are functionally similar. This is accomplished by classifying wetlands based on the hydrogeomorphic factors of geomorphic setting, dominant water source, and hydrodynamics.

The process of identifying functionally similar wetland classes is indeterminate. This is because the number of functionally similar wetland classes that can be identified depends on the size of the geographic area, the range of natural variation that is desired, and the subjective tendencies of different individuals to lump or split wetland types. Initially, Brinson (1993) identified five functionally similar "national wetland classes" in the conterminous United States. These were later expanded to seven national wetland classes including riverine, depression, slope, organic soil flat, mineral soil flat, estuarine fringe, and lacustrine fringe, as described in Table 4 (Smith et al. 1995, Brinson 1995). Each of the seven national wetland classes exhibits a reduced range of natural variation in function compared to all wetlands; however, each class still encompasses a range of natural variation in function that is greater than what is considered optimal for the assessment of wetland functions in the context of a 404 permit review. Consequently, it is necessary to further reduce the range of natural variation in function through a process of regionalization and subsequent application of the HGM Classification and, in some cases, other relevant factors at an intra-region scale to identify "wetland subclasses" (Table 5). Reducing the range of natural variation in function narrows the focus to 
Table 4. National Wetland Classes.

\begin{tabular}{|c|c|}
\hline Class & Definition \\
\hline Depression & $\begin{array}{l}\text { Depression wetlands occur in topographic depressions that allow the accumulation of surface } \\
\text { water. They are distinguished from Lacustrine Fringe wetlands by the lack of deep water areas } \\
\text { found in lakes, reservoirs, and other large bodies of water. Depressions may have any combina- } \\
\text { tion of inlets and outlets, or lack them completely. Potential water sources are precipitation, } \\
\text { overland flow, streams, or groundwater flow from adjacent uplands. The predominant direction of } \\
\text { flow is from the margins of the topographic depression to the lowest point in the depression. } \\
\text { Hydrodynamically, depressions exhibit vertical fluctuations with temporal scales ranging from a } \\
\text { few days to many months. Depressions lose water through intermittent or perennial outlets, } \\
\text { infiltration and percolation to subsurface layers, and evapotranspiration. Small or "micro" } \\
\text { depression areas that occur in association with other wetland classes, such as riverine or flat } \\
\text { wetlands, are generally not considered to belong to the depression class, but rather are } \\
\text { considered to be characteristic components of the wetland class in which they occur. Prairie } \\
\text { potholes, playa lakes, vernal pools, Carolina Bays, and cypress domes are common examples of } \\
\text { depression wetlands. }\end{array}$ \\
\hline $\begin{array}{l}\text { Tidal } \\
\text { Fringe }\end{array}$ & $\begin{array}{l}\text { Tidal fringe wetlands occur along coasts and estuaries under the influence of sea level and } \\
\text { tides. They intergrade landward with riverine wetlands where tidal influence diminishes and } \\
\text { channel flow becomes the dominant water source. Additional water sources may be } \\
\text { groundwater and precipitation. Because tidal fringe wetlands are frequently flooded and water } \\
\text { table elevations are controlled mainly by sea surface elevation, tidal fringe wetlands seldom dry } \\
\text { for significant periods. Tidal fringe wetlands lose water by tidal exchange, by overland flow to } \\
\text { tidal creek channels, and by evapotranspiration. Organic matter normally accumulates in higher } \\
\text { elevation marsh areas where flooding is less frequent and the wetlands are isolated from } \\
\text { shoreline wave erosion by intervening areas of low marsh or dunes. Spartina alterniflora salt } \\
\text { marshes are a common example of tidal fringe wetlands. }\end{array}$ \\
\hline $\begin{array}{l}\text { Lacustrine } \\
\text { Fringe }\end{array}$ & $\begin{array}{l}\text { Lacustrine fringe wetlands are adjacent to lakes, reservoirs, or other large bodies of water. } \\
\text { Sources of water are the adjacent body of water, precipitation and groundwater discharge } \\
\text { where lacustrine fringe wetlands intergrade with uplands or slope wetlands. Surface water flow } \\
\text { is bidirectional. Lacustrine fringe wetlands lose water by flow returning to the adjacent body of } \\
\text { water after flooding, and evapotranspiration. Organic matter may accumulate in areas } \\
\text { sufficiently protected from shoreline wave erosion. Unimpounded marshes of the Great Lakes } \\
\text { are an example of lacustrine fringe wetlands. }\end{array}$ \\
\hline Slope & $\begin{array}{l}\text { Slope wetlands are found on slightly sloping to steeply sloping land surfaces where } \\
\text { groundwater discharge or saturated overland flow occurs without channel formation. Slope } \\
\text { wetlands are distinguished from depression wetlands by the lack of a closed topographic } \\
\text { depression and the predominance of the groundwater water source. Precipitation is also a } \\
\text { potential source of water. Hydrodynamics are dominated by downslope, unidirectional flow of } \\
\text { water. Slope wetlands lose water primarily by saturated subsurface flows, surface flows, and by } \\
\text { evapotranspiration. Fens are a common example of slope wetlands. }\end{array}$ \\
\hline $\begin{array}{l}\text { Mineral } \\
\text { Soil } \\
\text { Flats }\end{array}$ & $\begin{array}{l}\text { Mineral soil flats are most common on relatively flat land surfaces such as interfluves, } \\
\text { extensive relic lake bottoms, or large alluvial terraces. The primary source of water is } \\
\text { precipitation. They receive virtually no groundwater discharge, which distinguishes them from } \\
\text { the depression and slope wetland classes. Dominant hydrodynamics are vertical fluctuations. } \\
\text { Mineral soil flats lose water by overland flow, infiltration, and percolation to subsurface layers, } \\
\text { and evapotranspiration. They are distinguished from non-wetland flats land surfaces by their } \\
\text { poor vertical drainage due to low permeability soils or impermeable layers (e.g., hardpans), slow } \\
\text { lateral drainage, and low hydraulic gradients. Pine flatwoods on hydric soils are an example of } \\
\text { mineral soil flat wetlands. }\end{array}$ \\
\hline
\end{tabular}




\begin{tabular}{|l|l|}
\hline Class & Definition \\
\hline $\begin{array}{l}\text { Organic } \\
\text { Soil } \\
\text { Flats }\end{array}$ & $\begin{array}{l}\text { Organic soil flats, or extensive peatlands, differ from mineral soil flats in part because their } \\
\text { elevation and topography are controlled by vertical accretion of organic matter. They commonly } \\
\text { occur on flat interfluves, but may also be located where depressions have become filled with } \\
\text { peat to form a relatively large flat surface. Precipitation is the dominant water source, while } \\
\text { water loss is via overland flow and infiltration and percolation to subsurface layers. Portions of } \\
\text { the Everglades and northern Minnesota peatlands are examples of organic soil flat wetlands. }\end{array}$ \\
\hline & $\begin{array}{l}\text { Riverine wetlands occur in floodplains and riparian corridors associated with stream channels. } \\
\text { The dominant sources of water are overbank or backwater flow from the stream channel. } \\
\text { Additional sources may be groundwater or overland flow from adjacent uplands, tributary inflow, } \\
\text { and precipitation. During overbank events, the hydrodynamics of riverine wetlands are } \\
\text { unidirectionally downstream. Headwater riverine wetlands often intergrade with slope, } \\
\text { depression, or flat wetlands as the stream channel diminishes. Riverine wetlands lose water via } \\
\text { the return of surface water to the stream channel, infiltration and percolation to subsurface } \\
\text { layers and the stream channel, and evapotranspiration. Bottomland hardwood forests on } \\
\text { floodplains are an example of riverine wetlands. }\end{array}$ \\
\hline
\end{tabular}

Adapted from Brinson (1993, 1995) and Smith et al. (1995).

Table 5. Potential Wetland Subclasses.

\begin{tabular}{|l|l|l|l|l|}
\hline \multicolumn{2}{|c|}{ Hydrogeomorphic Classification Factors } & \multicolumn{2}{c|}{ Potential Wetland Subclasses } \\
\hline $\begin{array}{l}\text { Geomorphic } \\
\text { Setting }\end{array}$ & Water Source & Hydrodynamics & Eastern USA & $\begin{array}{l}\text { Western } \\
\text { USA/Alaska }\end{array}$ \\
\hline Depression & $\begin{array}{l}\text { Groundwater or } \\
\text { interflow }\end{array}$ & Vertical fluctuation & $\begin{array}{l}\text { Prairie pothole marshes, } \\
\text { Carolina bays }\end{array}$ & Vernal pools \\
\hline Fringe (tidal) & Ocean & $\begin{array}{l}\text { Bidirectional, } \\
\text { horizontal }\end{array}$ & $\begin{array}{l}\text { Chesapeake Bay and Gulf of } \\
\text { Mexico tidal marshes }\end{array}$ & $\begin{array}{l}\text { San Francisco Bay } \\
\text { marshes }\end{array}$ \\
\hline $\begin{array}{l}\text { Fringe } \\
\text { (lacustrine) }\end{array}$ & Lake & $\begin{array}{l}\text { Bidirectional, } \\
\text { horizontal }\end{array}$ & Great Lakes marshes & $\begin{array}{l}\text { Flathead Lake } \\
\text { marshes }\end{array}$ \\
\hline $\begin{array}{l}\text { Slope } \\
\text { Groundwater } \\
\text { soil) }\end{array}$ & $\begin{array}{l}\text { Unidirectional, } \\
\text { horizontal }\end{array}$ & Fens & Avalanche chutes \\
\hline $\begin{array}{l}\text { Flat (organic } \\
\text { soil) }\end{array}$ & Precipitation & Vertical & Wet pine flatwoods & Large playas \\
\hline Riverine & $\begin{array}{l}\text { Overbank flow from } \\
\text { channels }\end{array}$ & $\begin{array}{l}\text { Unidirectional, } \\
\text { horizontal }\end{array}$ & $\begin{array}{l}\text { Bottomland hardwood } \\
\text { forests }\end{array}$ & $\begin{array}{l}\text { Peatlands over } \\
\text { permafrost }\end{array}$ \\
\hline
\end{tabular}

Adapted from Smith et al. (1995) and Rheinhardt et al. (1997).

those functions performed by a particular wetland subclass and the characteristics and processes that influence those functions. This simplifies the development of assessment procedures and models and makes it possible for the procedures to be both rapid, and capable of measuring changes in wetland functions at a level of resolution appropriate for assessing project impacts or restoration success. 


\section{Regionalization}

In the HGM Approach, a region is defined as a geographic area that is relatively homogenous with respect to climate, geology, physiography, geomorphology, soils, and a variety of other factors that can influence how wetlands function. Historically, regionalization was the process of identifying the boundaries of one or more regions in the geographic "area of interest" where a guidebook is being developed. In some cases, the area of interest will be predetermined by the underlying reason for developing a guidebook. For example, the area of interest may correspond to the geographic extent of a particular type of wetland that is subject to a disproportionate level of development pressure (Bauder et al. 2009). It may correspond to an area that will be impacted by a large project such as a highway project, conservation plan, or urban development. Alternatively it may correspond to a hydrologic, political, or regulatory boundary such as a watershed, county, state, national park, or Corps of Engineers District (Smith and Klimas 2002).

Regardless of the underlying reason, there are a number of regionalization systems that can potentially serve as a starting point for identification of regions. These systems are based on various criteria such as resource type (USDA 2006), geomorphology (Saucier 1995), physiography (Fenneman and Johnson 1946), climate (Trewartha 1943), hydrology (U.S. Geological Survey (USGS) 1982), vegetation (Küchler 1964, 1970), or ecoregions (Omernik 1987, Bailey 1980, Commission for Environmental Cooperation (CEC) 1997). In some parts of the country, existing wetland classifications can serve as a starting point for identifying wetland subclasses (Stewart and Kantrud 1971, Golet and Larson 1974, Wharton 1978). A good example is the vernal pool regions identified by Keeler-Wolf et al. (1998) based on geomorphology, soils, and vegetation in California.

Perhaps the most familiar system for regionalization in the United States is the ecoregion developed for the U.S. Environmental Protection Agency (USEPA) (Omernik 1987, 1995, 2004; Bryce et al. 1999). Ecoregions are

described as areas with relative consistency in terms of the geomorphic, climatic, hydrologic, and biological conditions and processes (Omernik 1987). Ecoregion maps are available at four levels of detail (http://www.epa.gov/ wed/pages/ecoregions.htm). Level I divides North America into 15 broad regions (e.g., Great Plains, Eastern Temperate Forests). Level II divides the Level I regions into 50 smaller regions. Level III divides the Level II regions into 
182 smaller regions, and Level IV divides the Level III regions at a state level while maintaining consistency across adjoining state boundaries (Figure 3). Ecoregion maps include a brief description of the classification units shown on the map. Omernik (1995), Omernik and Gallant (1990), and Gallant et al. (1989) provide arguments for using USEPA ecoregions as the basis for regionalization.

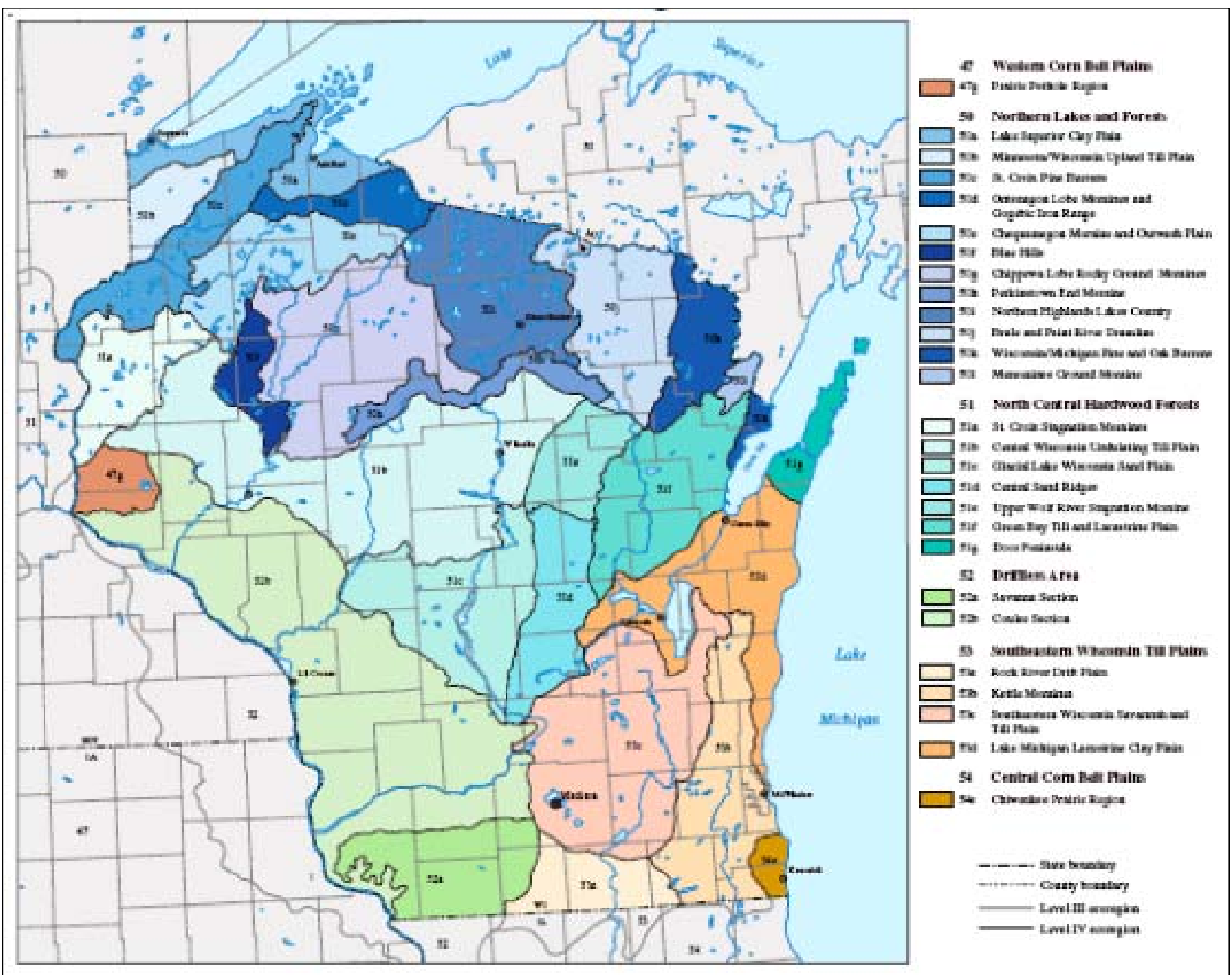

Figure 3. Example of Level III and Level IV U.S. Ecoregions for Wisconsin.

The U.S. Forest Service (USFS) has developed a different set of ecoregions for the United States (Bailey 1980, Bailey et al. 1994). This regionalization system is also hierarchical, successively dividing landscapes from the Domain through Divisions, Provinces, Sections, and Subsections (Figure 4). It is based on a similar suite of structural characteristics and processes as the USEPA ecoregions, but places more emphasis on the natural vegetation cover in establishing unit boundaries. The USFS ecoregions are accompanied by a narrative description of each Section and 


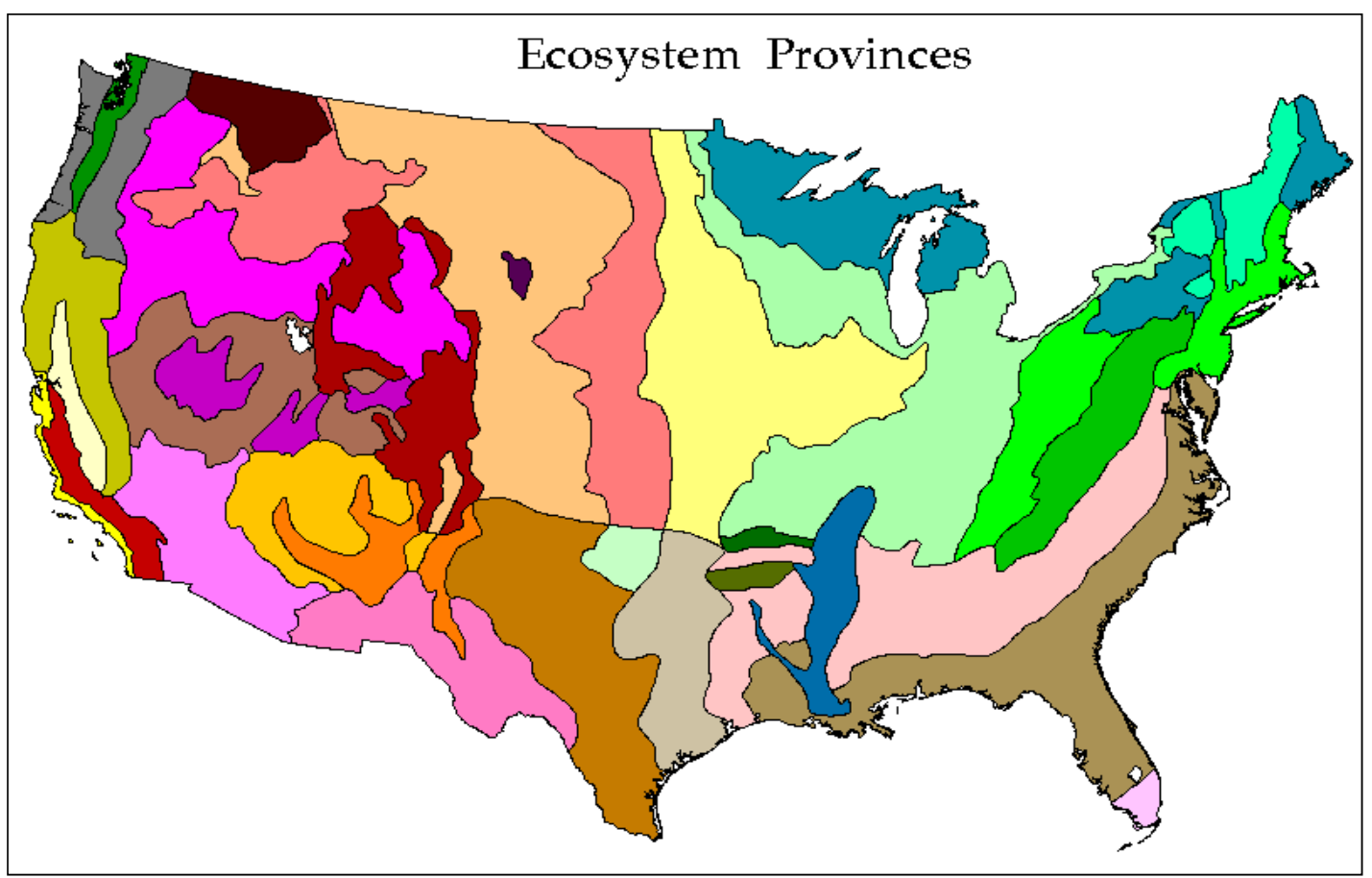

Figure 4. Example of an ecological unit at the Province level of the U.S. Forest Service national regionalization system.

Subsection classification unit that can be helpful in understanding the variation among wetlands when defining wetland subclasses. In addition, the USFS ecoregion maps are accompanied by detailed ecological descriptions that can be useful for understanding the fundamental controls on the occurrence and characteristics of wetlands, and therefore useful in identifying appropriate criteria for establishing wetland subclasses. The USFS ecoregion maps and descriptions are available at: http://www.fs.fed.us/land/pubs/ecoregions/.

The Natural Resource Conservation Service (NRCS) is the federal agency with a resource-based mapping mission and is responsible for producing spatially explicit soil surveys for every county in the nation. Unlike the USFS and USEPA hierarchical ecoregion systems, which begin with large land units that are split into increasingly smaller units, the NRCS system begins with the soil map unit, which can be less than an acre in size, and then aggregates the units to successively larger areas known as Land Resource Units (LRU), Major Land Resource Areas (MLRA), and Land Resource Regions (LRR) (Figure 5). These areas generally reflect resource concerns, soil groups, hydrologic units, resource use, topography, other landscape features, and human considerations affecting use and treatment needs. This system is used for regionalizing hydric soil indicators and supplements to the Wetland Delineation Manual. 


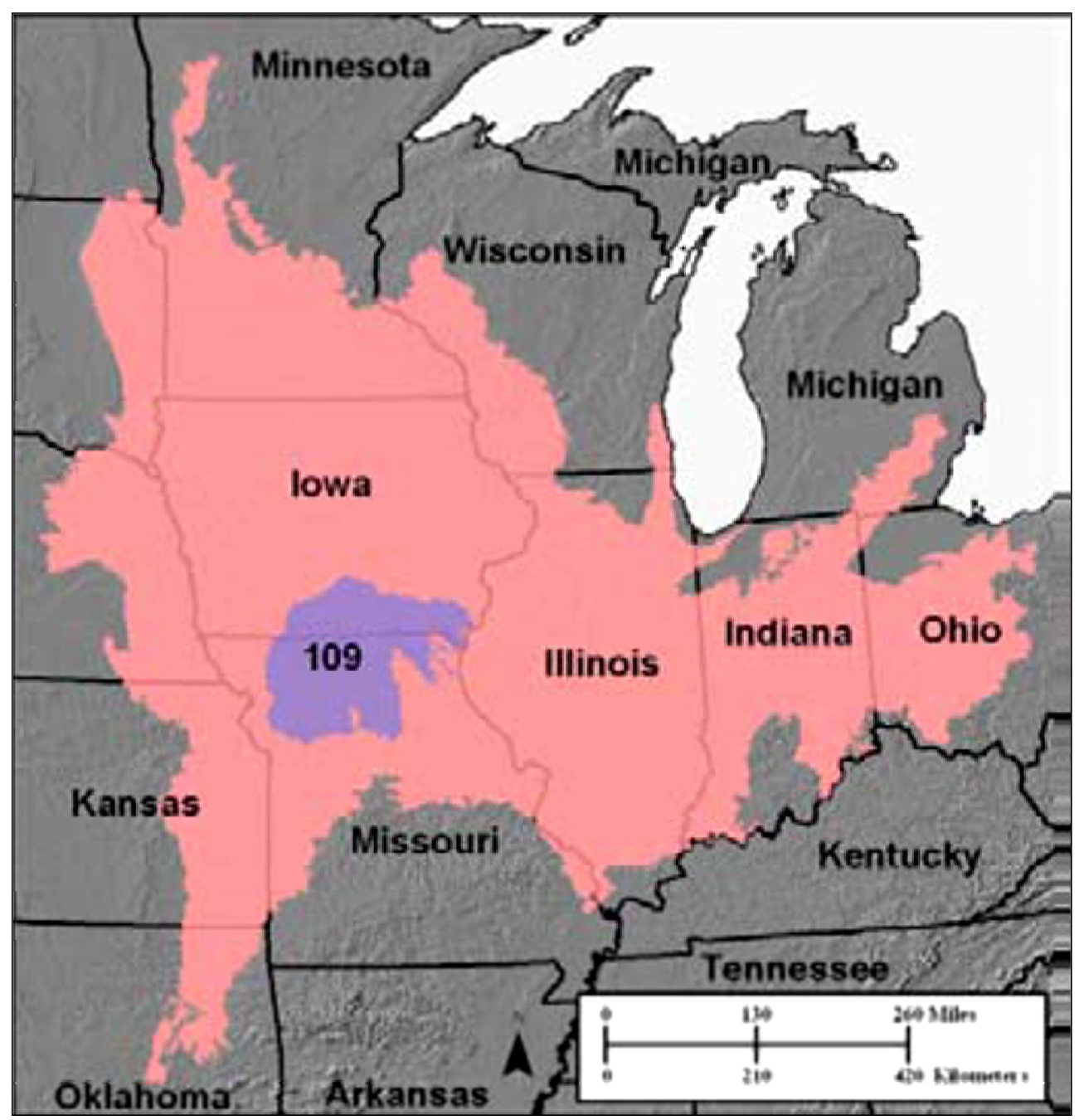

Figure 5. Example of Land Resource Regions (pink) and Major Land Resource Areas (purple).

The NRCS system maintains an emphasis on soils, limitations for agricultural use, natural vegetation, and hydrology. In fact, all three systems have utility under particular circumstances and can be used together in order to take advantage of the strengths of each. More information on this regionalization system is available in Agriculture Handbook 296 (USDA-NRCS 2006), and at:

http://www.glti.nrcs.usda.gov/technical/publications/nrph.html.

There are advantages to using established regionalization systems as a starting point in identifying regions. First, they are widely recognized, based on an explicit set of criteria, and readily available in geospatial formats. Second, to a large extent, they are based on natural ecological boundaries that reflect the fundamental physical and biological conditions 
that control how wetlands function. As indicated, the regionalization systems do differ to some degree in terms of what is emphasized, and the level of detail provided with regard to ecosystem characteristics. The USEPA ecoregions, the USFWS ecoregions, and the NRCS resource areas are compared in Agriculture Handbook 296 (USDA-NRCS 2006), which includes an appendix that cross-references the NRCS resource areas with USEPA and USFS ecoregions.

In practice, when developing a guidebook, the process of regionalization involves reviewing the maps of existing regionalization systems and determining which ecoregion, resource area, or other unit overlaps with the area of interest. Begin with the smallest unit (e.g., USEPA Level IV Ecoregions, USFS Subsections, and NRCS Land Resource Units), and determine where the area of interest intersects one or more units of the regionalization system. If the area of interest intersects a single unit, select that unit as the "preliminary region." If the area of interest intersects multiple units of the regionalization system, identify all of them as the preliminary region. Most guidebooks select a preliminary region that corresponds to the boundaries of the units prescribed by a regionalization system. Ultimately, however, the boundaries of the preliminary region may need to be modified to correspond to political, jurisdictional, project, other non-ecological boundaries, or time and resource constraints when the reference domain is identified (see the section titled "Define the Reference Domain" below).

\section{Define wetland subclasses}

Once a preliminary region has been identified, the next step is to classify each of the wetland types under consideration in terms of the HGM Classification. As with the geographic area of interest, the number of wetland types under consideration may be predetermined by the underlying reasons for developing the guidebook. In other cases the assessment team will have considerable flexibility in how many wetland types are to be considered.

Begin this step by comparing the characteristics of each wetland type under consideration with the descriptions of the wetland classes shown in Table 4. Based on this comparison, assign the appropriate national wetland class to each wetland type. Once the appropriate national wetland class has been identified, apply the HGM Classification factors at an intra-regional scale to define wetland subclasses that exhibit the desired range of natural variation 
in function. In the development of a guidebook, the wetland subclass is the endpoint of the HGM classification, and represents a range of natural variation in function that is suitable for developing assessment models that are sensitive to changes in function. Examples of potential wetland subclasses are shown in Table 5 (Smith et al. 1995, Rheinhardt et al. 1997). This step is necessary because, within a region, national wetland classes can exhibit a wide natural range in function. It may be necessary to reduce the range of natural variation so that assessment models are sensitive to the types of changes in function that occur in the wetland under consideration. For example, depression wetlands can be isolated with no inflow or outflow, or connected with inflows, outflow, or both (Bauder et al. 2009). They can have a primary surface or groundwater source, or occupy various landscape positions such as plateaus, interfluves, or valley floors (Hollands 1987). Similarly, the tidal fringe wetlands can exhibit different tidal regimes and salinity gradients (Shafer and Yozzo 1998). Slope wetlands exhibit differences in the degree of slope, landscape position, surface versus groundwater source of water, or other factors. Wetlands in the riverine class exhibit differences due to water source, hydrologic regime, watershed position, stream order, watershed size, channel gradient, or floodplain width (Smith and Klimas 2002, Klimas et al. 2009).

One of the strengths of the HGM Classification is that it focuses on physical site characteristics when defining a wetland subclass rather than the vegetation cover, or other characteristics that can change over time. This allows wetlands to be classified consistently regardless of the successional stage or current land use. However, in certain situations, factors other than geomorphic setting, water source, and hydrodynamics may be used to identify a wetland subclass. For example, in certain cases, specific "indicator" plant species or "community types" can serve as a fundamental site characteristic. A specific example occurs in the case of determining the acidity or nutrient levels of a water source in organic soil flat or slope wetlands, or when a mineral soil flat wetland has limiting levels of salt accumulation (Klimas et al. 2005). Similarly it is common for the primary source of water in a wetland to be used as a criterion for identifying a wetland subclass. In these situations it is important, whenever possible, that criteria represent stable, long-term conditions that do not change relatively rapidly. It is also important that criteria are based on features that can be measured consistently from maps, aerial photos, field observations, or other sources. 


\section{Define the reference domain}

The reference domain is the geographic portion of the preliminary region where reference wetland data are collected and subsequently used to calibrate the assessment models in a guidebook. Ideally, the reference domain coincides with the full geographic extent of the preliminary region and the wetland subclass. However, in practice this is not always possible. Frequently the reference domain includes only a portion of the full geographic extent of a wetland subclass. This is usually due to constraints related to agency jurisdictional authority, the extent of management responsibility, lack of time and resources, or other factors.

There are at least three different scenarios for defining the reference domain. The first scenario utilizes a "top-down" approach in which the full geographic extent of the wetland subclass is surveyed, and then representative reference wetlands are identified and sampled across the full geographic extent of the wetland subclass. This approach is robust, but can be time and resource intensive, especially if the geographical extent of the wetland subclass is large.

In the second scenario, the reference domain is predetermined by an existing political, regulatory, or hydrologic boundary such as a watershed, county, state, or Corp of Engineers District. For example, the State of Arkansas developed the HGM Regional Guidebook for the Delta Region of Arkansas (Klimas et al. 2004), and defined the reference domain as only that portion of USEPA Level III Ecoregion 73 (Mississippi Alluvial Plain) that occurred within the boundaries of the state of Arkansas (Figure 6), even though the ecoregion extends into parts of six other states (Figure 7). It is important when using this approach to verify that the reference domain captures the full natural range of variation exhibited by the wetland subclass throughout its geographical extent. Without this verification, use of the guidebook may be restricted to the geographic area corresponding to the reference domain.

The third scenario is a "bottom-up" approach in which a relatively small geographic area (e.g., a county, watershed, project area) is identified as the initial reference domain based on initial objectives or responsibilities, and subsequently expanded over time as additional reference wetland data are acquired. Again, it is important when using this approach to verify that the relatively small reference domain does in fact capture the full range of natural variation exhibited by the wetland subclass throughout its 


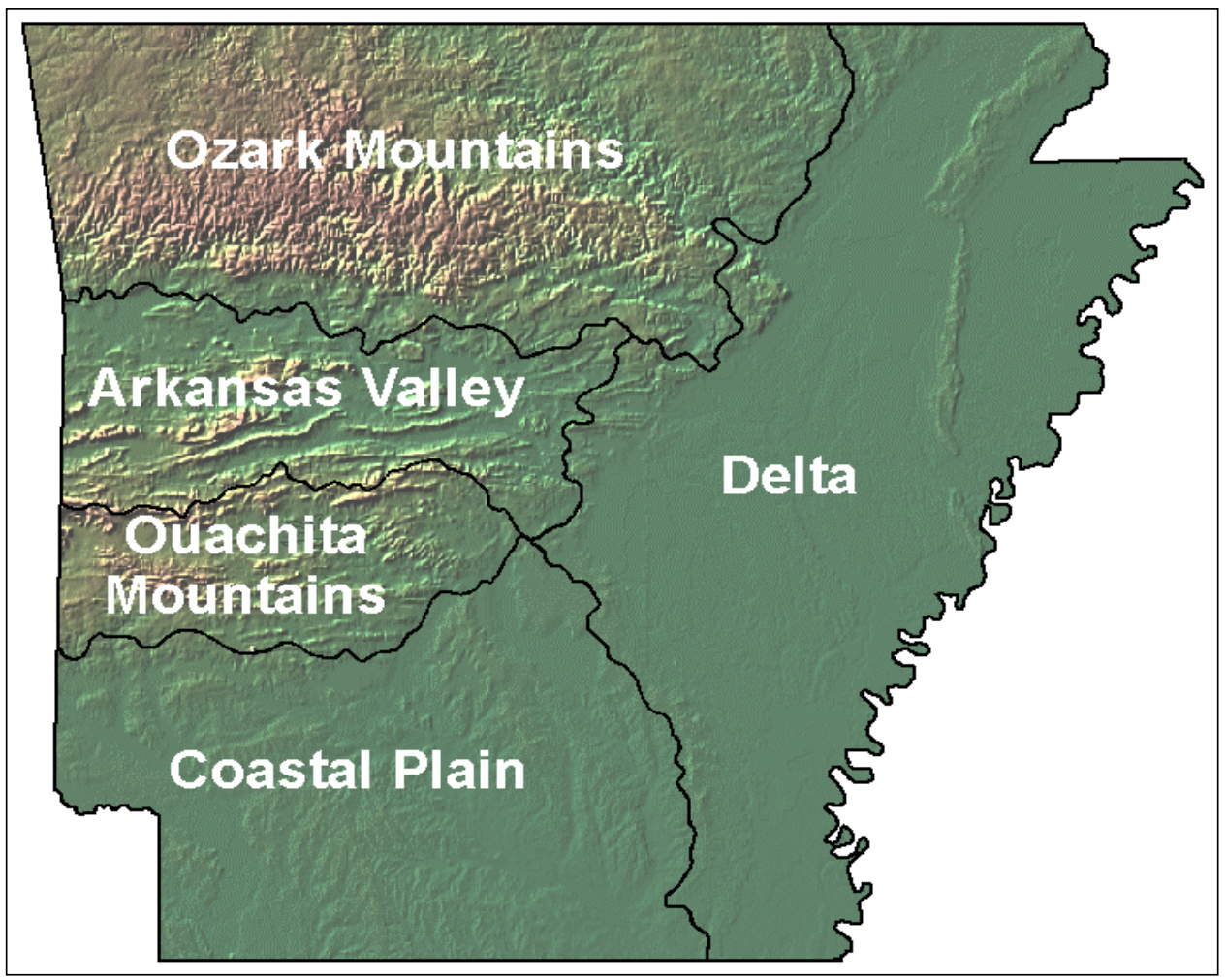

Figure 6. Reference domain for the Delta Region of Arkansas (Klimas et al. 2004).

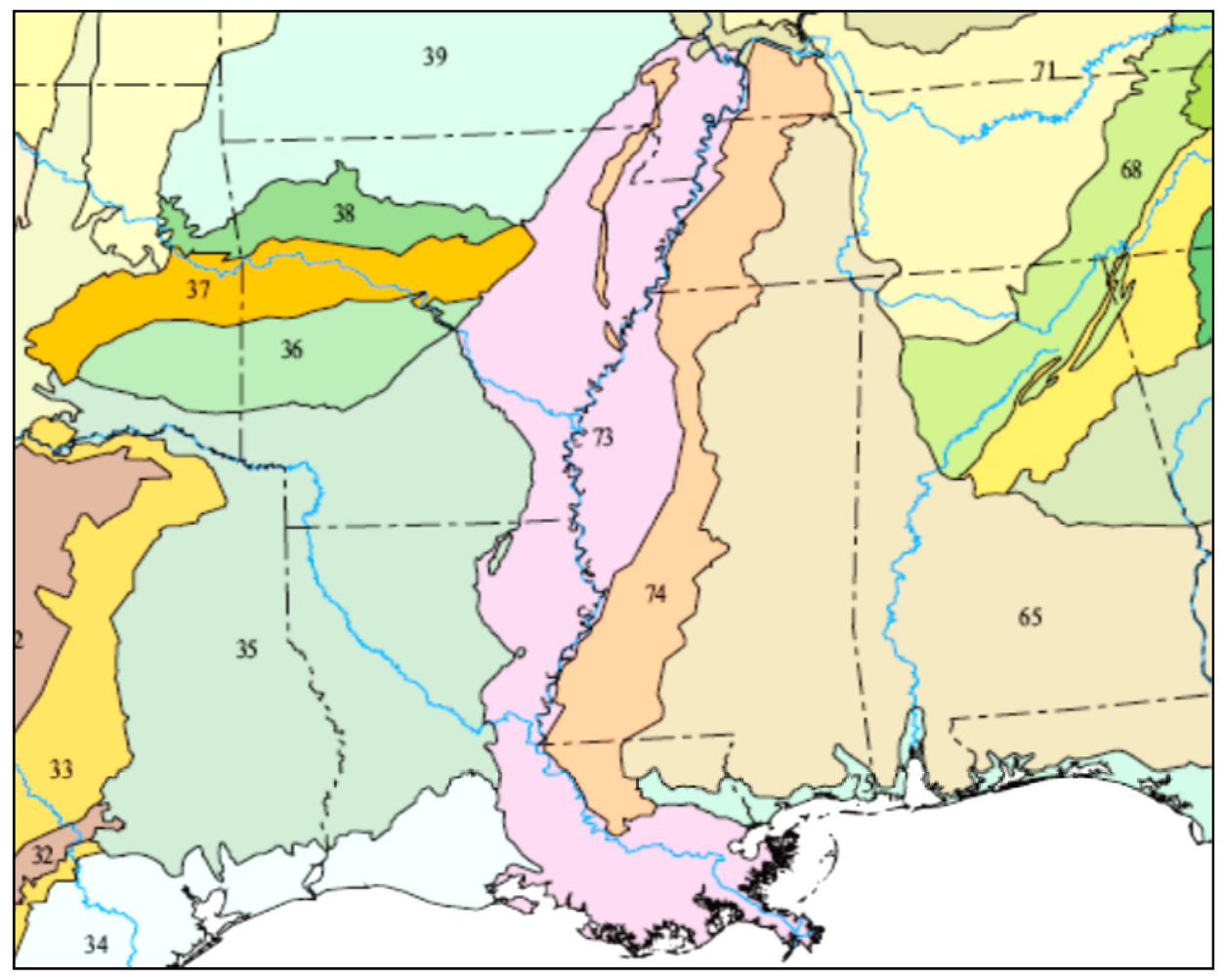

Figure 7. EPA Level III Ecoregion 73 (Mississippi Alluvial Plain). 
geographical extent. Without this verification, use of the guidebook will necessarily be restricted to the geographic area corresponding to the reference domain. The advantage of the bottom-up approach is that it requires the least initial amount of time and resources, and is amenable to expansion as time and resources become available. This may be necessary if the initial geographic extent of the reference domain fails to capture the full range of natural variation exhibited by the wetland subclass.

\section{Number of wetland subclasses in a guidebook}

Most of the guidebooks that have been developed and published to date (Figures 1 and 2) consider a single wetland subclass. However, a few guidebooks include multiple subclasses. Examples are the guidebook for the southern portion of the Yazoo River watershed in Mississippi (Smith and Klimas 2002), and guidebooks for several different regions of Arkansas (Klimas et al. 2004, 2005, 2006). The Arkansas Multi-Agency Wetland Planning Team website provides useful information on how regions were defined, how the HGM Classification was applied to identify wetland subclasses in each region, as well as links to the guidebooks developed for different regions in Arkansas (http://www.mawpt.org/wetlands/classification/ classification.asp).

The main advantage of developing a guidebook for a single wetland subclass is that it requires the least amount of time and resources. The disadvantage is that it does not provide a comprehensive picture of wetland subclasses in the area of interest, and cannot be used to assess wetland functions for regulatory or planning efforts that involve multiple wetland types. The advantage of a guidebook that addresses multiple wetland subclasses is that it can be applied consistently across multiple wetland types. As indicated, the impetus for developing a guidebook that addresses multiple wetland subclasses often originates from planning and regulatory agencies intending to use guidebooks to inventory, manage, and restore wetlands for projects that encompass large geographic areas. The disadvantage of a guidebook that addresses multiple wetland subclasses is that it can require considerable time and resources, particularly when the area of interest is large or the diversity of wetland types is great. One possible solution is to develop multiple guidebooks that address a single wetland subclass according to a prioritization scheme as funding becomes available. The disadvantage of this strategy is that the individual guidebooks will necessarily include some redundancies. 
Guidebooks that address multiple wetland subclasses require the development of a comprehensive classification of wetlands in the region of interest. The classification needs to be constructed carefully to avoid overlap or gaps among wetland subclasses. A useful way to assure that the classification is complete and exclusive is to construct a dichotomous key. An example is provided in the guidebook for the West Gulf Coastal Plain region of Arkansas (Klimas et al. 2005). Figure 8 shows a dichotomous key to wetlands classes in the region, and Figure 9 a dichotomous key of wetland subclasses within each wetland class. Note that the key includes an additional level of classification, the criteria "community type," for certain wetland subclasses. In this case, community types were identified to assure that special sites or those with unique floristic characteristics (e.g., beaver complexes and bayheads), were clearly identified so that when reference wetland data were collected and analyzed, it could be determined whether variable metrics should be scaled differently for different community types. This demonstrates the utility of developing a comprehensive classification system in the region of interest.

\begin{tabular}{|c|}
\hline to Wetland Classes of the Coastal Plain Region of Arkansas \\
\hline $\begin{array}{l}\text { 1. Wetland is within the } 5 \text {-year floodplain of a stream } \\
\text { 1. Wetland is not within the } 5 \text {-year floodplain of a stream............................................. }\end{array}$ \\
\hline $\begin{array}{l}\text { 2. Wetland is not in a topographic depression or impounded. } \\
\text { 2. Wetland is in a topographic depression or impounded. }\end{array}$ \\
\hline 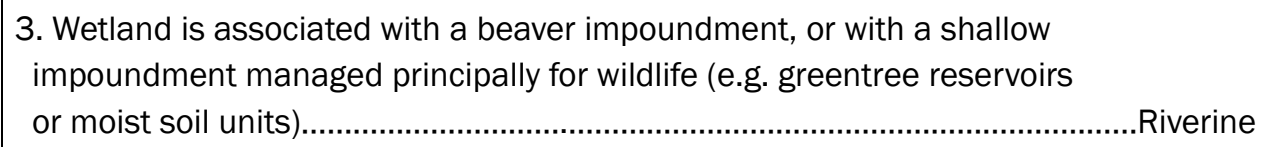 \\
\hline 3. Wetland is an impoundment or depression other than above \\
\hline $\begin{array}{l}\text { 4. Wetland is associated with a water body that has permanent open water more } \\
\text { than } 2 \mathrm{~m} \text { deep in most years.......................................................................Finge }\end{array}$ \\
\hline 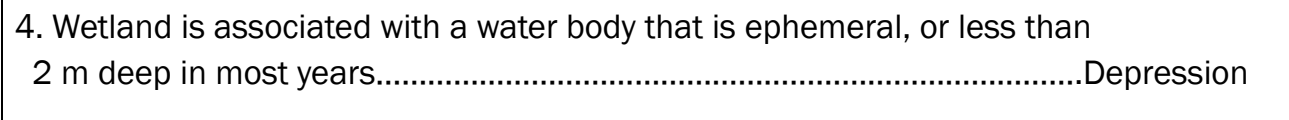 \\
\hline 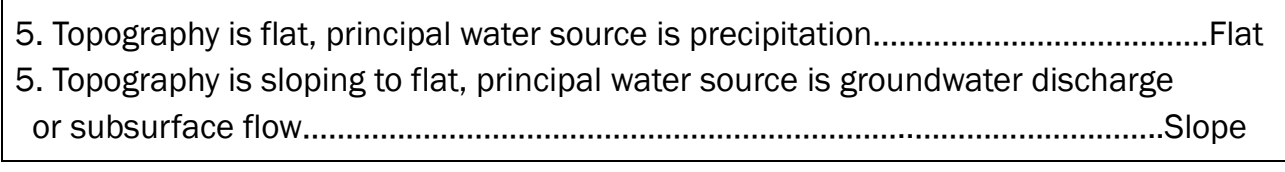 \\
\hline
\end{tabular}

Figure 8. Key to the Wetland Classes in the Coastal Plain region of Arkansas (Klimas et al. 2005). 


\begin{tabular}{|c|c|c|}
\hline \multicolumn{3}{|c|}{ Key to Regional Wetland Subclasses and Community Types in the Coastal Plain Region of Arkansas } \\
\hline Flat Class & Subclass & Community Type \\
\hline $\begin{array}{l}\text { 1. Soil reaction circumneutral to alkaline (lake bed } \\
\text { deposits) }\end{array}$ & Alkali Flat & alkali wet prairie \\
\hline 1. Soil reaction acid & Non-Alkali Flat (2) & \\
\hline 2. Vegetation dominated by graminoids & & wet tallgrass prairie \\
\hline \multicolumn{3}{|l|}{ 2. Vegetation dominated by woody species } \\
\hline 2a. Vegetation dominated by pine & & pine flat \\
\hline 2b. Vegetation dominated by post oak & & post oak flat \\
\hline $\begin{array}{l}\text { 2c. Vegetation dominated by hardwoods other than } \\
\text { post oak }\end{array}$ & & hardwood flat \\
\hline Depression Class & Subclass & Community Type \\
\hline $\begin{array}{l}\text { 1. Depression not subject to direct stream flooding } \\
\text { during a } 5 \text {-year event; precipitation, runoff, and } \\
\text { groundwater are the dominant inflows }\end{array}$ & Unconnected Depression & $\begin{array}{l}\text { unconnected alluvial } \\
\text { depression }\end{array}$ \\
\hline $\begin{array}{l}\text { 1. Depression has significant direct stream inflows and } \\
\text { outflows relative to stored volume and/or is influenced } \\
\text { by overbank or backwater flooding during a 5-year event }\end{array}$ & Connected Depression & floodplain depression \\
\hline Fringe Class & Subclass & Community Type \\
\hline 1. Wetland on the margin of a man-made reservoir & Reservoir Fringe & reservoir shore \\
\hline $\begin{array}{l}\text { 1. Wetland on the margin of water body other than a } \\
\text { reservoir }\end{array}$ & 2 & \\
\hline $\begin{array}{l}\text { 2. Water body is subject to stream flooding during } \\
5 \text {-year flood events }\end{array}$ & $\begin{array}{l}\text { Connected Lacustrine } \\
\text { Fringe }\end{array}$ & connected lake margin \\
\hline $\begin{array}{l}\text { 2. Water body not subject to flooding during a 5-year } \\
\text { event }\end{array}$ & $\begin{array}{l}\text { Unconnected Lacustrine } \\
\text { Fringe }\end{array}$ & unconnected lake margin \\
\hline Riverine Class & Subclass & Community Type \\
\hline 1. Wetland associated with low-gradient stream & 3 & \\
\hline 1. Wetland associated with mid-gradient stream & Mid-gradient Riverine (2) & \\
\hline $\begin{array}{l}\text { 2. Water source primarily overbank flooding or lateral } \\
\text { saturation }\end{array}$ & & mid-gradient floodplain \\
\hline 2. Wetland an impoundment & Riverine Impounded (4) & \\
\hline 3. Wetland not an impoundment & Low-gradient Riverine (5) & \\
\hline 3. Wetland an impoundment & Riverine Impounded (4) & \\
\hline 4. Wetland impounded by beaver & & beaver complex \\
\hline $\begin{array}{l}\text { 4. Wetland impounded for wildlife management } \\
\text { (greentree reservoirs and moist soil units) }\end{array}$ & & $\begin{array}{l}\text { managed wildlife } \\
\text { impoundments }\end{array}$ \\
\hline
\end{tabular}

Figure 9. Key to the wetland subclasses and community types in the Coastal Plain region of Arkansas (Klimas et al. 2005) (continued). 


\begin{tabular}{|l|l|l|}
\hline $\begin{array}{l}\text { 5. Wetland a prairie, substrate is a Pleistocene lake } \\
\text { beach deposit }\end{array}$ & sand prairie \\
\hline $\begin{array}{l}\text { 5. Wetland dominated by woody or non-prairie species, } \\
\text { site not a beach deposit }\end{array}$ & 6 & \\
\hline $\begin{array}{l}\text { 6. Water source primarily overbank flooding (5-year } \\
\text { zone) that falls with stream water levels, or lateral } \\
\text { saturation from channel flow }\end{array}$ & & low-gradient overbank \\
\hline $\begin{array}{l}\text { 6. Water source primarily backwater flooding or } \\
\text { overbank flows (5-year zone) that remain in the } \\
\text { wetland due to impeded drainage after stream water } \\
\text { levels fall }\end{array}$ & low-gradient backwater \\
\hline Slope Class & Subclass & Community Type \\
\hline $\begin{array}{l}\text { 1. Sweetbay (Magnolia virginiana) common or } \\
\text { dominant }\end{array}$ & Non-calcareous Slope & bayhead \\
\hline $\begin{array}{l}\text { 1. Sweetbay not common, wetland dominated by other } \\
\text { species }\end{array}$ & Non-calcareous Slope & perennial seep \\
\hline
\end{tabular}

Figure 9. (concluded).

Finally, guidebooks that address a single wetland subclass have greater utility if they provide context in the form of a general discussion of all of the wetland subclasses that occur in the region. Developing a comprehensive classification for the region of interest requires the assessment team to clearly define differences between wetland subclasses. This exercise is encouraged because it often reveals gaps or overlaps among wetland subclasses that can be missed when a single wetland subclass is considered in isolation. Ultimately a comprehensive classification will help the end user to apply the guidebook appropriately, and facilitates the development of additional guidebooks.

\section{Characterize the wetland subclass}

Each guidebook includes a chapter that characterizes the wetland subclass or subclasses under consideration (Table 2). The objective of this section is to define the geographic area where the guidebook is applicable, and to characterize the wetland subclass in terms of physical setting, structural characteristics, processes, and common types of cultural alteration. The characterization chapter includes sections on climate, geology, geomorphic setting, hydrologic regime, soils, vegetation, and wildlife. Because guidebooks are designed to assess changes to existing wetlands, the characterization chapter should include a discussion of commonly encountered natural and anthropogenic disturbances. Each of these topics is discussed in greater detail below. 


\section{Climate}

A characterization of the climate within the reference domain should be included in the guidebook, with a focus on the availability of water to wetlands. Specifically, the section should discuss the typical precipitation patterns with respect to type (e.g., snow, rain), quantity, and timing, whether extended periods of water deficit are common, and whether a significant fraction of the total precipitation is delayed from reaching wetlands until spring because it is stored as snow. The discussion should identify the seasons of greatest and least precipitation, temperature extremes, and the extent of the dormant and growing seasons. Usually, a narrative description is sufficient and lengthy tables of rainfall and temperature data are not needed. The frequency and severity of damaging storms, such as hurricanes, tornados, and ice storms, should also be described. Climate data can be obtained from NRCS and the National Oceanic and Atmospheric Administration (NOAA) websites (http://www.wcc.nrcs.usda.gov/climate/wets_doc.html, www.noaa.gov).

\section{Geologic and geomorphic setting}

The geologic setting can control a variety of aspects affecting wetland structure and function. A basic description of the origins and characteristics of the fundamental structural elements of the reference domain landscape, such as major rock formations and glacial deposits, should be presented. This provides a basis for any subsequent discussions of how wetlands are influenced by surface topography, groundwater movement, water chemistry, and similar issues. Basic geologic structure can directly affect the occurrence and characteristics of wetlands, such as where contacts between certain sedimentary layers consistently discharge groundwater and support slope wetlands. More commonly, geology affects wetlands in terms of coarse-scale influences such as elevation, slope, and parent materials. The more subtle characteristics of surface topography and soils that influence wetlands are the result of geomorphic processes.

Geomorphic features are surface landforms that primarily reflect the erosion and deposition processes controlled by topography. In the context of wetlands, the most familiar geomorphic settings are those created by the meandering movement of streams, which erode, transport, and deposit sediments in characteristic patterns on floodplains. The different ways in which the resulting point bars, natural levees, abandoned channel segments, and other distinctive features receive and store water directly dictate 
how the wetlands they support are classified. Other familiar types of geomorphic settings that influence wetland occurrence and characteristics are: the deltaic and coastal deposits that support intertidal salt marshes, the wind-deflated depressions where "pothole" and playa wetlands occur, and the various basins and depressions found in glacial till.

Basic descriptive information on the major geologic formations and their distribution is typically available from state agencies that regulate mining and water supplies, or from natural resource agencies. Geomorphic data are less available, because they are generated primarily to support specific project planning objectives, most often those related to engineering or archeological surveys. Therefore, where detailed geomorphic mapping is available, it is rarely comprehensive and may cover only some portion of the reference domain, such as a proposed highway alignment. However, even piecemeal mapping can be helpful if the accompanying reports describe the origins and characteristics of the principal geomorphic features in such a way that they can be recognized when they are encountered elsewhere in the reference domain.

Whether or not detailed maps can be obtained, a good basic description of the geologic and geomorphic settings that occur in the reference domain can usually be assembled from the federal ecoregion classification publications referenced previously. In addition, the Geological Society of America has published a comprehensive overview of all of the major geomorphic systems of North America (Graf 1987); it provides considerable detail on both the geology and geomorphology of each major physiographic region on the continent.

Regardless of the source of the information, it is helpful to include graphics that clearly indicate where on the landscape each wetland subclass is likely to occur. One simple way to do this is in the form of a block diagram. Block diagrams can range from a simple representation of the topographic setting of a single wetland subclass relative to other wetland and non-wetland communities (Figure 10), to more comprehensive depictions of the geologic and geomorphic settings within the reference domain and their relationship to the occurrence of the major wetland subclasses (Figures 11 and 12).

\section{Hydrologic regime}

The hydrology of wetlands in the reference domain should be described at a level of detail sufficient to allow guidebook users to understand and 


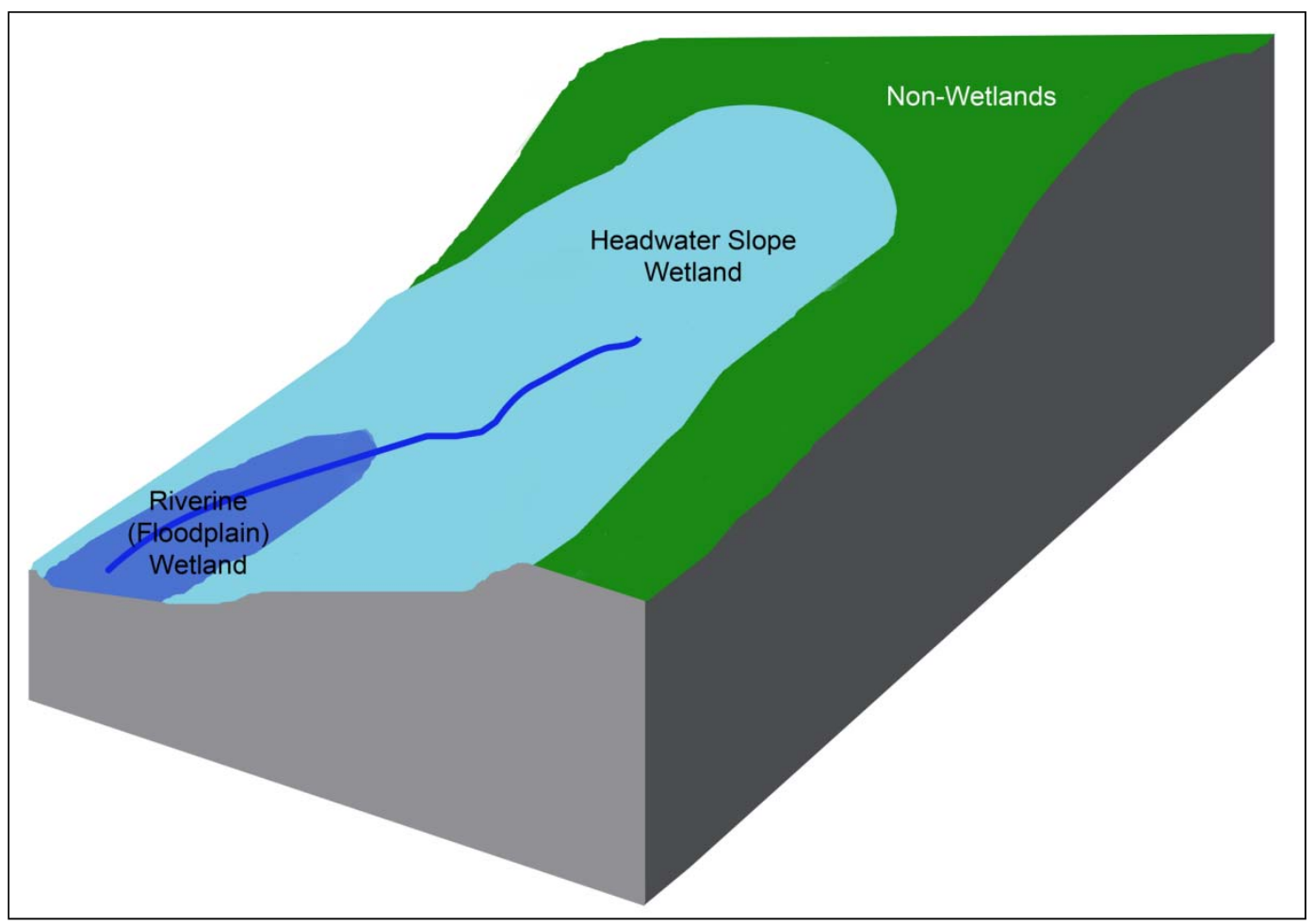

Figure 10. Generalized landscape position of Headwater Slope wetlands in southeast Mississippi and southwest Alabama (Noble et al. 2007).

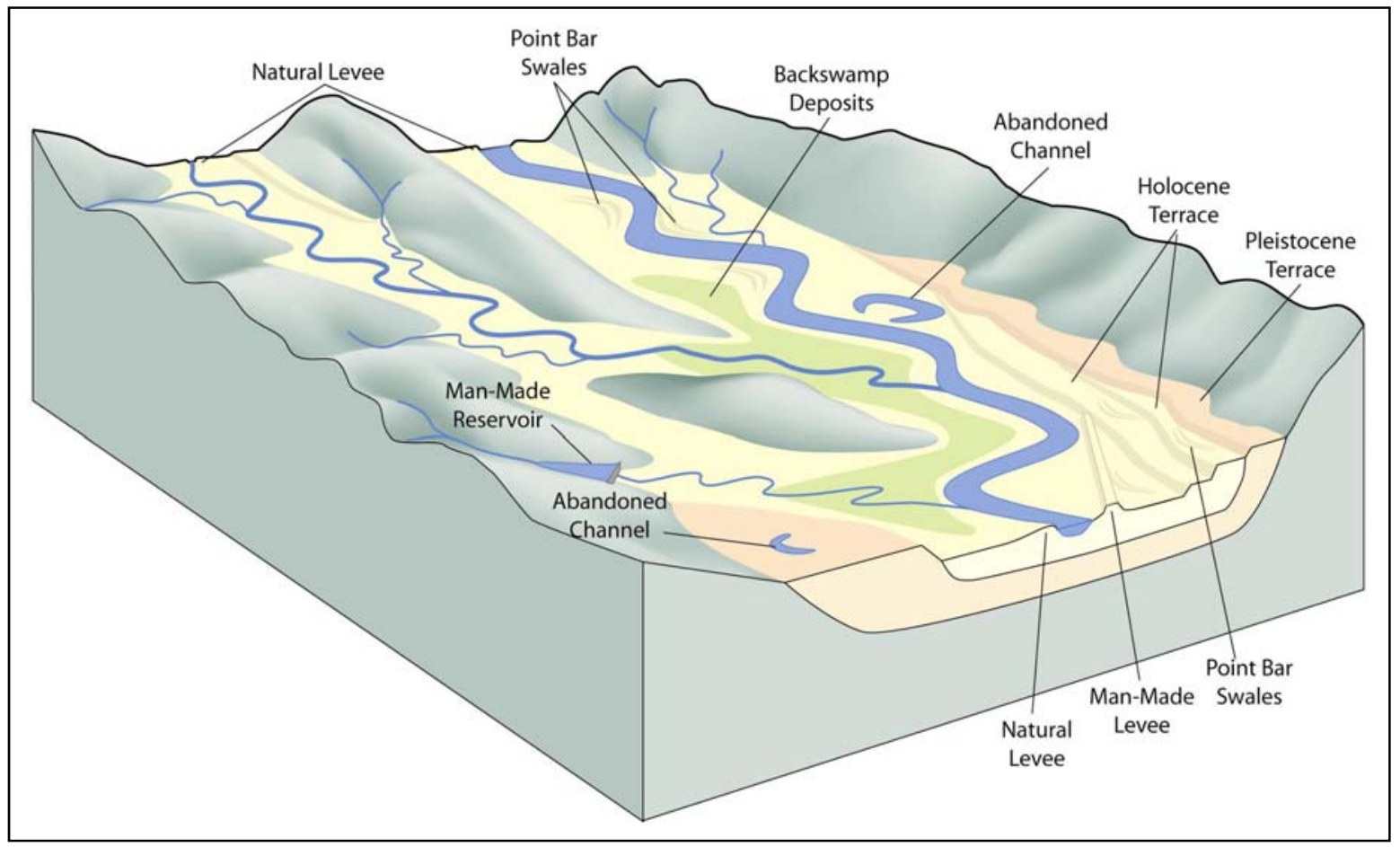

Figure 11. Typical form and locations of geomorphic and man-made features within the Arkansas Valley Wetland Planning Region (Klimas et al. 2008). 


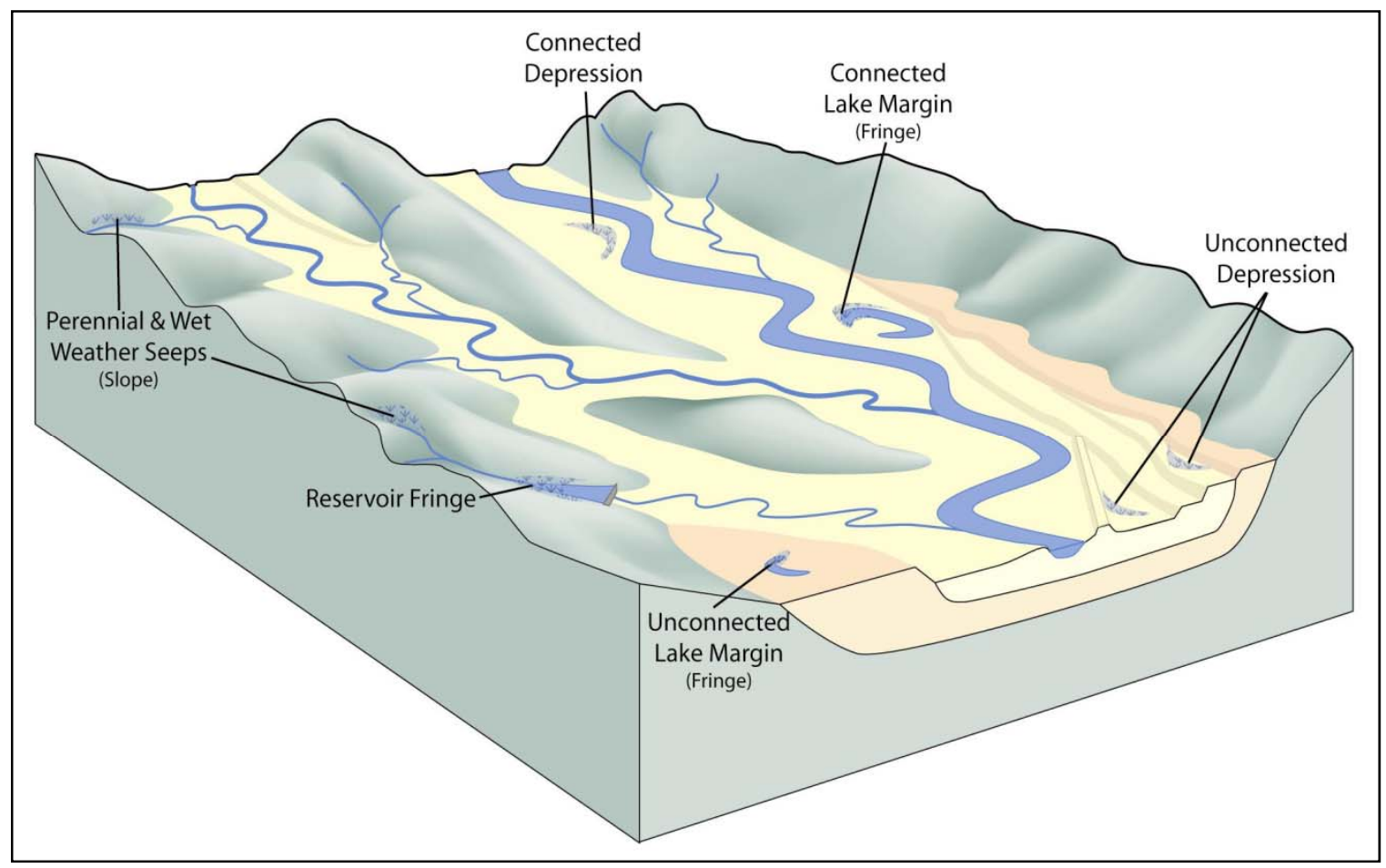

Figure 12. Typical landscape positions of depression, fringe, and slope wetlands in the Arkansas Valley Wetland Planning Region (Klimas et al. 2008).

recognize differences that influence the designation of wetland subclasses. In addition, the reader should be able to find and use whatever hydrologic data will be needed to apply the wetland assessment models presented later in the guidebook.

At the most fundamental level, hydrologic regimes are either tidal or nontidal, and the two are considered differently. Tidal wetlands - those areas alternately exposed and inundated due to lunar-influenced tides - are generally defined in terms of the vertical and horizontal range of tidal influence, the salinity of the tidal waters, the seasonal and short-term hydroperiods, and similar measures. Regional patterns reflecting these types of variables are incorporated into the designation of ecoregions under the classification systems described previously, and are developed in some detail in the national wetland classification system presented by Cowardin (1979). One or more of these sources should be consulted to isolate the variables of interest in the reference domain, and to help resolve classification issues that relate to the transition from coastal tidal systems to inland riverine systems. Coastal rivers in the continental United States have some part of their lower reaches subject to tidal influence such that water and salinity levels fluctuate. In places where the coastal plain is narrow and abuts much steeper terrain, such as southern California, the tidal zone 
within rivers is short and often has high salinities. Where the coastal plain is broad and flat, as it is in much of the Carolinas and Virginia, tidal influence frequently extends dozens of miles upstream, resulting in a long, continuous gradient of change from saline coastal fringe wetlands to freshwater tidal riverine systems. How and where to designate changes in wetland class and subclass along this gradient requires a good understanding of the particular river systems involved, and the guidebook user will benefit most from specific maps that designate break points between types a priori. The issues involved in developing and applying HGM procedures in tidally influenced systems are discussed in the national HGM tidal fringe guidebook (Shafer and Yozzo 1998).

Hydrologic considerations pertinent to the classification and assessment of freshwater wetlands are diverse and can be complex. Water sources influencing the characteristics of wetlands may include rainfall, groundwater discharge, lake level fluctuations, runoff from uplands, flooding from stream channels, and prolonged soil saturation due to gradual snowmelt. Most commonly, several water sources will be present and have varying levels of influence on the wetland. Under HGM classification guidelines, it is important to understand the predominant source of water controlling wetland characteristics, as well as the hydrodynamics of the system. At minimum, an HGM guidebook should include a discussion of the major types of water sources present in a region, the characteristics of the stream network, any major water control structures that influence river flows, and similar issues.

The types of information normally available with respect to freshwater hydrology can be difficult to interpret in terms of small wetland communities and areas that are not directly connected to river channels or bodies of water. The U.S. Geological Survey has extensive information on groundwater resources (http://water.usgs.gov/ogw/) that should be consulted when developing general regional characterizations. However, much of this data concerns large, deep aquifers, while groundwater influences on wetlands tend to involve shallow aquifers and localized storage. The parameter of primary interest with respect to groundwater is whether or not the flow is perennial in most years or seasonal ("wet weather seeps"), and often that has to be evaluated in the field on the basis of the dominant vegetation or local knowledge. 
Historically, perennial groundwater sources have been used as potable water sources by local residents, and evidence of that use is apparent in the form of pipes and excavated pools. Once a groundwater discharge point has been identified, additional examples of the slope wetlands it supports usually can be found by tracking the geologic source, which is often a contact point between two rock formations.

Another important water source that can be difficult to describe thoroughly is flooding. The common perception - that rainfall within a watershed causes streams to spill over their banks, with the water gradually draining back into the channel at a predictable rate - describes just one of many possible flooding patterns. On large rivers, flooding may occur in response to rainfall or snowmelt in tributary basins many hundreds of miles away. Smaller tributaries also can flood without any substantial rain in their drainage basins via backwater effects, where high water on a larger stream causes tributary flows to back up and move into floodplains. Return flows to the channel can occur rapidly once stream levels fall, but many floodplains include extensive backswamp areas, where return flows are impeded by natural levee deposits. Floodwaters can remain in these areas for long periods, until evapotranspiration removes the standing water. The possible variations in the origins and progress of flood events make it difficult to characterize the hydrologic regime in wetland subclasses that are found in floodplains, and most HGM documents dealing with riverine wetlands have adopted flood frequency as the most accessible indicator of overall flooding patterns. In some areas, flood frequency maps have been generated to support watershed planning or construction projects. Where such maps are not available, they can be generated through a careful analysis of gage data and floodplain surface elevations, or by examining aerial photos taken during flood events of known frequency. Less rigorous options for describing flooding can include simple local knowledge of flood behavior on particular streams, and indirect indicators on the floodplain, such as wrack and water marks on trees. The latter are rarely satisfactory for establishing frequency or duration of flooding, and simply indicate that a site is subject to inundation.

\section{Soils}

Soils information is of interest in the wetland characterization portion of the HGM guidebook primarily as it reflects drainage conditions and the potential for water to pond on the surface for extensive periods of time. Unlike geomorphic and hydrologic data, soils data are readily available for 
essentially all areas of the United States, and detailed descriptions, chemical and physical characteristics, and soils mapping are available online for download or direct interactive use (http://websoilsurvey.nrcs.usda.gov/app/; http://datagateway.nrcs.usda.gov). These sites and other resources they link to are highly useful for characterizing wetlands, as they directly address key characteristics of wetland soils and the associated water regime and vegetation. Because soils are an integral consideration in wetland jurisdictional determinations under the Clean Water Act and various farm programs, special attention is paid to hydric soils. HGM guidebooks should include discussion of the hydric soils associated with each wetland subclass of interest, and should direct guidebook users to consult soils maps to help locate and classify wetlands in the landscape.

\section{Vegetation and wildlife}

A discussion of the biota of the reference domain accomplishes two objectives: describe the range of conditions normally encountered in the wetland subclass or subclasses covered by the guidebook and place those wetlands in the context of the larger ecosystem. Details pertaining to the vegetation located within the wetland subclass should be derived directly from the HGM field data. The dominant vegetation of each subclass should be discussed, as well as common variants that occur, such as early developmental stages, and by potential impacts of invasive species.

A discussion of the characteristic biota of other wetland subclasses and uplands in the region is a useful part of an HGM guidebook, but is not integral to its application. An overview of the vegetation and wildlife communities of the region is helpful in orienting the guidebook user to the region's diversity and where the wetlands of interest fit into the larger ecosystem. The aforementioned ecoregion classification systems, particularly the Forest Service system, provide good overviews of the major plant communities. More detailed descriptions of wildlife communities can be found in state resource agency documents and web sites, as well as numerous technical publications.

\section{Natural and anthropogenic disturbances}

This discussion is an important part of the overall characterization of the ecosystem, and should be presented in an HGM context, with an emphasis on disturbances that influence the hydrogeomorphic setting, processes, and structural characteristics of wetlands. This approach will also provide 
some perspective on the concept of the "reference standard" wetland by emphasizing that the particular wetland communities the guidebook describes have experienced a variety of natural and human impacts.

As a first step, this section of the guidebook should discuss the occurrence and impacts of severe storms, particularly hurricanes and ice storms, if they periodically affect the reference domain. Such storms can greatly alter the structure of wetland systems over large areas, and changes to wetlands caused by these catastrophic events should be noted. For example, trees in wetlands tend to be subject to extensive blowdown during hurricanes, which can alter the structure of floodplain forests for a century or more. Similarly, ice storms cause canopy damage in forested wetlands, which changes the structure of the understory and increases woody debris on the forest floor. In coastal environments, hurricanes can destroy or alter large areas of coastal marsh and submerged aquatic plant beds. These and similar natural events are part of the "background" condition of wetlands in a region, and should be recognized as such in the guidebook.

The discussion of human influences should mention any pre-settlement Native American activities that may have altered wetlands extensively, such as fire and agriculture, as well as early historic development of timber and mining operations, and the work of local drainage districts. However, most of the focus of this section should be on the major changes in land use and hydrologic processes that have influenced wetlands in the modern era. Data on long-term trends in wetland acreage losses, by wetland type, can be found in Dahl (1990, 2000, 2005). Local and regional sources can provide additional information on land use and development trends; and data related to agricultural acreage can be found on the NRCS websites cited previously.

Information on hydrologic changes can be more difficult to locate. The USGS provides water resource summaries for all parts of the United States at: http://water.usgs.gov/, and much of this information is relevant to understanding the constraints and stresses placed on wetlands in some regions. However, specific information on the effects of levee systems, navigation channels and harbors, reservoirs, diversion projects, irrigation return water, and similar changes to the landscape are not generally available from any one central repository, but can be assembled from individual agency sources. Relatively simple summaries of many engineering projects can be found on agency websites, and in published 
documents. For example, most Corps of Engineers District websites maintain a description of the major water projects in the district that report dates of construction, project purposes, and operational schedules. By consulting these and other sources, the hydrologic characteristics of the reference domain can be described in terms of how human activities may have altered the natural flow of water through the reference domain. 


\section{Conceptualize Assessment Models}

\section{Introduction}

Assessment models are developed in three distinct phases in the HGM Approach. During the first phase, assessment models are conceptualized based on the personal knowledge and experience of the assessment team members, the literature, and available data. The conceptualization process begins with the selection of functions to represent the regional wetland subclass, and continues with the identification of model variables to represent the ecosystem attributes and processes that influence how the various functions are performed. Once ecosystem functions and model variables have been identified, select metrics for measuring variables, defining the relationship between metrics and functional capacity, and finally, defining the relationship between variables and functional capacity. The second and third phases of assessment model development involve testing, calibration, and validation of the assessment models conceptualized during the first phase. The second and third phases are discussed in Chapter 5, "Calibrate, Verify, and Field-Test Assessment Models," and Chapter 7, "Validate Assessment Models."

\section{Overview of assessment models}

A model is a simplified representation of a system that is used to understand the structure and functions of the system, and in some cases to predict how the system will respond to different conditions and perturbations. Models range in complexity from a simple map to three-dimensional numerical simulations of water flow, ocean currents, and weather (Hall and Day 1977, Jorgensen 1988, Mitsch et al. 1988). The complexity of a model may reflect the inherent complexity of a system, the level of detail at which information about a system is gathered, the objectives of the model builder, or the needs of model end-users. A model that is appropriate for meeting the needs of end-users is not necessarily complex (Skellum 1969). However, it should be recognized that simplification, which is often a legitimate and necessary part of model development, is only achieved at the cost of a reduction in model accuracy and precision (Levins 1996). Ultimately, the degree of simplification is dictated by the characteristics of the system under consideration, and the intended application of the model. 
In the HGM Approach, models are representations of how wetlands, belonging to a regional wetland subclass, perform specific functions. The assessment models are developed as simple mathematical equations that prescribe how assessment model variables (hereafter variables) are combined to derive a functional capacity index (FCI). Models with a similar format have been variously described in the literature as multiple criterion models (Smith and Theberge 1986), composite indices (Ott 1978), or multimetric models (Barbour et al. 1995).

In the assessment model, variables are measurable attributes, characteristics, and processes of the wetland ecosystem, and the surrounding landscape, that influence the capacity of a wetland to perform a function. The output from the model is an FCI, which represents the capacity of a wetland to perform a function relative to other wetlands in the same regional wetland subclass. The FCI, like all indices, is a ratio of the value of interest divided by a standard of comparison (Inhaber 1976). The standard of comparison for an FCI is the range of conditions that occur in reference standard wetlands. Reference standard wetlands are the subset of minimally disturbed (Stoddard et al. 2006) reference wetlands, that by definition achieve the highest, sustainable levels of function, across the suite of functions ascribed to a regional wetland subclass (see Chapter 4, "Reference Wetlands and Reference Wetland Data").

Variables are measured using a variable metric (hereafter metric). The metric that is chosen, and the scale of measurement (e.g., nominal, ordinal, interval, or ratio) and units for each metric depend on the type of variable under consideration. For example, the variable "tree biomass" could be measured using the ratio scale metric "basal area" in units of centimeters, or another metric indicative of tree biomass. Similarly, "hydrologic regime" is a variable that reflects the influence of hydrology on wetland functions. One of many possible metrics for hydrologic regime is the ratio scale metric frequency of flooding expressed as a return interval in the units of years.

In order to combine variables in the assessment model, metrics must be transformed into a comparable, unitless value, or variable subindex (hereafter subindex) (Schuster and Zuuring 1986, Smith and Theberge 1986). The transformation is based on what has been called a value function (O'Banion 1980), scalar (Westman 1985), or a "normalization and standardization" procedure (Barbour et al. 1995). Subindices are assigned a 
value ranging between 0.0 and 1.0 based on an explicitly defined relationship between the metric value and functional capacity (Figure 13). The range of metric values exhibited by reference standard wetlands are assigned a subindex of 1.o. As metric values deviate from the range of conditions exhibited in reference standard wetlands, a lower subindex is assigned. The deviation from the reference standard condition is attributed to a disturbance capable of decreasing wetland function in the wetland proper or the surrounding area. By convention, at the low end of the subindex range, highly disturbed wetlands that no longer perform a function, but retain the potential to recover through natural processes or restoration activities, are assigned a subindex of 0.1 (Figure 13). For example, the subindex assigned to tree basal area in an agricultural field is 0.1 because, while there are no trees currently present, tree basal area has the potential to increase over time through vegetation succession or restoration activities such as seeding or planting. Similarly, by convention, in highly disturbed wetlands where a disturbance is considered to be permanent and the likelihood of recovery or restoration improbable, a subindex of 0.0 is assigned. For example, the subindex assigned to tree basal area at a developed site (e.g., buildings, roads, parking lots, and other infrastructure) is o.o because it is unlikely that trees will be reestablished in the foreseeable future. Similarly, if a floodplain is isolated from an adjacent stream by levees, a subindex of 0.0 is assigned to the frequency of flooding metric because a normal hydrologic regime will no longer occur unless the levee is removed.

\section{Select wetland functions}

Prior to conceptualizing assessment models, the assessment team must select a suite of functions that will be ascribed to the regional wetland subclass. Wetland functions are the normal or characteristic activities that occur in wetland ecosystems, or simply the things that wetlands do. The selection of functions is challenging because wetlands perform a wide variety of functions, from simple to complex. For example, the reduction of nitrate to gaseous nitrogen is a function performed by wetlands when aerobic and anaerobic conditions exist in the presence of denitrifying bacteria. Nutrient cycling represents a complex wetland function that involves many components and processes. The objective is to identify a suite of functions that together represent the full range of characteristics and processes that occur in the regional wetland subclass and, if sustained, ensure the integrity of the wetland ecosystem. 


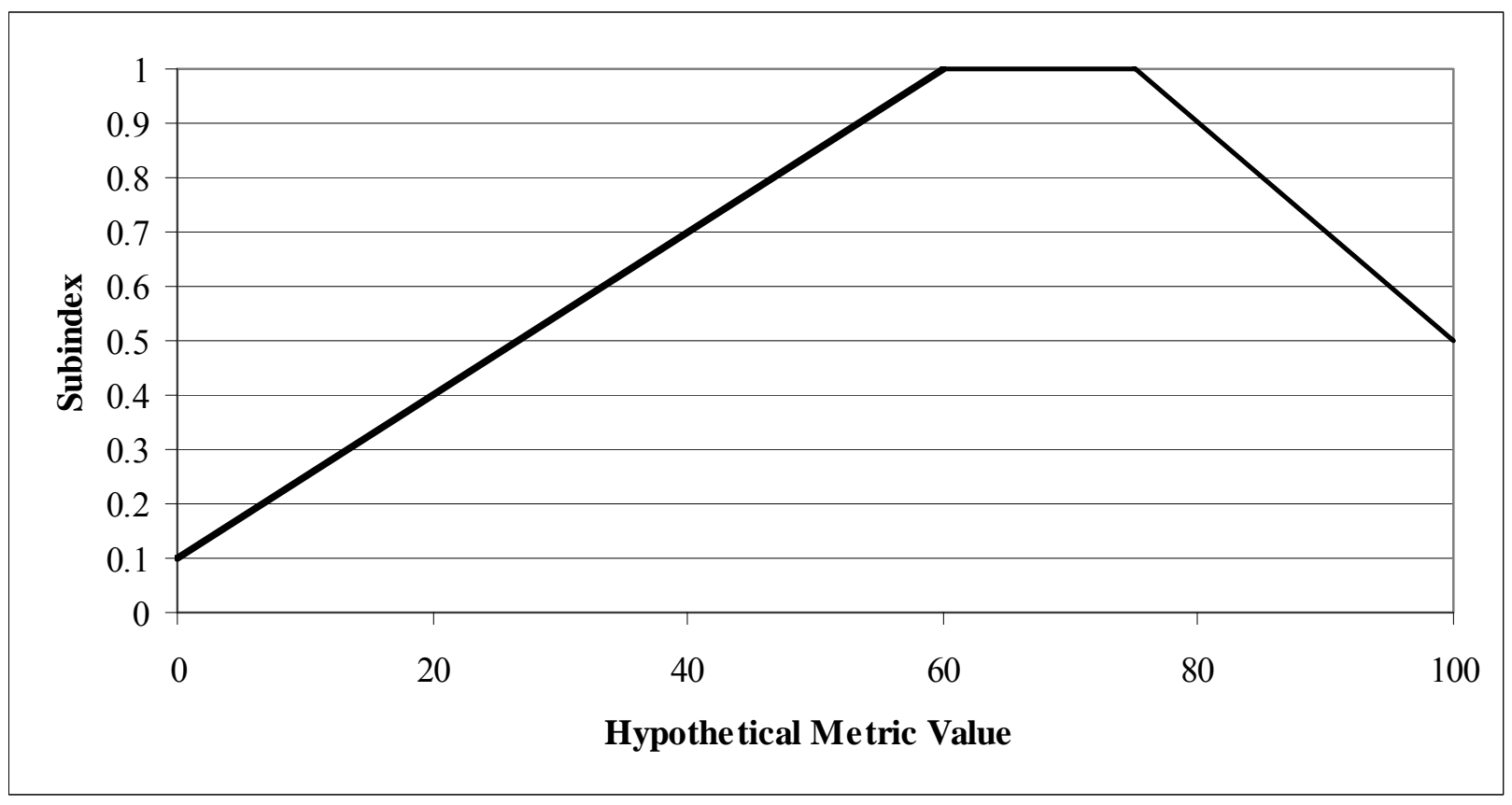

Figure 13. Relationship between hypothetical metric values and the variable subindex.

A useful starting point in selecting functions is the published regional guidebooks available online at: http://el.erdc.usace.army.mil/wetlands/hgmhp.html. These regional guidebooks define and describe functions selected for specific regional wetland subclasses, outline the criteria used in the selection process, explain why a function was selected, and reference the sources of information used during the selection process. In addition to published regional guidebooks, it is useful to review other procedures that have been developed to assess wetland functions. Procedures by Larson (1976), Euler et al. (1983), Hollands and Magee (1985), Ammann et al. (1986), Adamus et al. (1987), Ammann and Stone (1991), and Bartoldus et al. (1994) have identified and described a variety of wetland functions, and provide different perspectives on the selection of wetland functions. There is also an extensive literature on wetland functions that may be useful in identifying the functions performed by specific regional wetland subclasses (Greeson et al. 1979, Sather et al. 1984, Conservation Foundation 1988, Hubbard 1988, Novitski et al. 1997, Leibowitz 2003).

After reviewing published regional guidebooks and other literature on wetland functions, the assessment team should begin selecting functions. Functional selection should consider the ecosystem context in which functions are performed, the role of value in selecting functions, the hierarchy of functions, the resources available for conducting functional 
assessments, and the need to define selected functions in a concise and quantifiable way. Each of these considerations is discussed below.

\section{The ecosystem context}

Ecosystems are normally characterized in terms of their structural components and the processes that link these components (Bormann and Likens 1969). Structural components of the ecosystem and the surrounding landscape, such as plants, animals, detritus, soil, water, and the atmosphere, interact through a variety of physical, chemical, and biological processes such as the movement of air and water and the flow of energy and nutrients. Understanding how the structural components of the ecosystem and the surrounding landscape are linked together by processes serves as the basis for assessing ecosystem functions. In selecting wetland functions it is important to remember that functions cannot be assessed, mitigated, restored, enhanced, or traded independently. Rather, they represent interdependent activities that take place in the context of a complex integrated ecosystem.

Selecting a suite of functions to represent the range of characteristics and processes that occur in wetland ecosystems is consistent with the use of reference standard wetlands as the standard of comparison for calibrating assessment models. Reference standard wetlands are minimally disturbed wetland ecosystems in minimally disturbed landscapes that by definition achieve the highest sustainable level of function across the entire suite of functions performed by the regional wetland subclass. The highest sustainable level of function across an entire suite of functions often represents a lower level of function than is possible when considering a single wetland function in isolation from other functions. For example, under certain disturbance regimes, wetlands can capture enough sediment to fill the wetland, or retain floodwater long enough that the characteristic plant community is replaced. Under this type of scenario, while the wetland is performing a high level of a single function, it is often unsustainable and may lead to the elimination of other functions performed by the wetland. The use of the highest sustainable level of function across the entire suite of functions as a standard of comparison serves to ensure the integrity of the overall wetland ecosystem.

\section{The role of value}

Another challenge in selecting a suite of functions is the role "value" plays in the selection process. Value was defined by Smith et al. (1995) as the relative importance of something to an individual or group. For the assessment 
team, the "something" is wetland functions. The HGM Approach was not designed to assign value to wetland functions. It purposely avoids this objective, and focuses instead on supplying the technical information that is needed to quantify wetland functions.

It is, however, important to recognize that there is often an implicit bias to favor wetland functions with direct and immediate benefits to humans. For example, floodwater storage is one of the most commonly identified wetland functions because it is easily linked to images of flood victims and the large economic losses that are incurred as a result of flooding. At the other end of the spectrum, however, are wetland functions that provide important, but less direct and immediate, benefits. Wetlands, for example, function as carbon sinks by removing carbon dioxide from the atmosphere and storing it in living and dead plant biomass (Raich and Schlesinger 1992). This function has been implicated in the stabilization of atmospheric greenhouse gases (Gorham 1994), but since the benefits are less direct and immediate, it is rarely selected as a function for assessment. The purpose of this discussion is not to discourage the selection of functions that provide direct and immediate benefits, but rather to encourage consideration of wetland functions whose benefits, although less direct and immediate, may play an important role in maintaining the integrity of wetland ecosystems.

\section{Hierarchy of functions}

The HGM Approach defines wetland functions as the activities that normally occur in wetland ecosystems, or simply, the things that wetlands do (Smith et al. 1995). Wetland functions result from the interaction between the attributes of the wetland, its watershed, and the surrounding landscape (e.g., geomorphic setting, landscape position, watershed size), the structural components of the wetland ecosystem (e.g., plants, animals, soil, water, and the atmosphere), and the processes that link these structural components (e.g., overbank flooding, evapotranspiration, chemical reactions in the soil, predation, and the capture of light energy).

This rather broad definition makes it possible to identify a large number of functions for any particular regional wetland subclass. In selecting functions, one way to deal with the large number of functions is to think of wetland functions in terms of a hierarchy that begins with very general functions at the highest level and increasingly more specific and detailed functions at lower levels. For example, in Figure 14 element cycling is a 


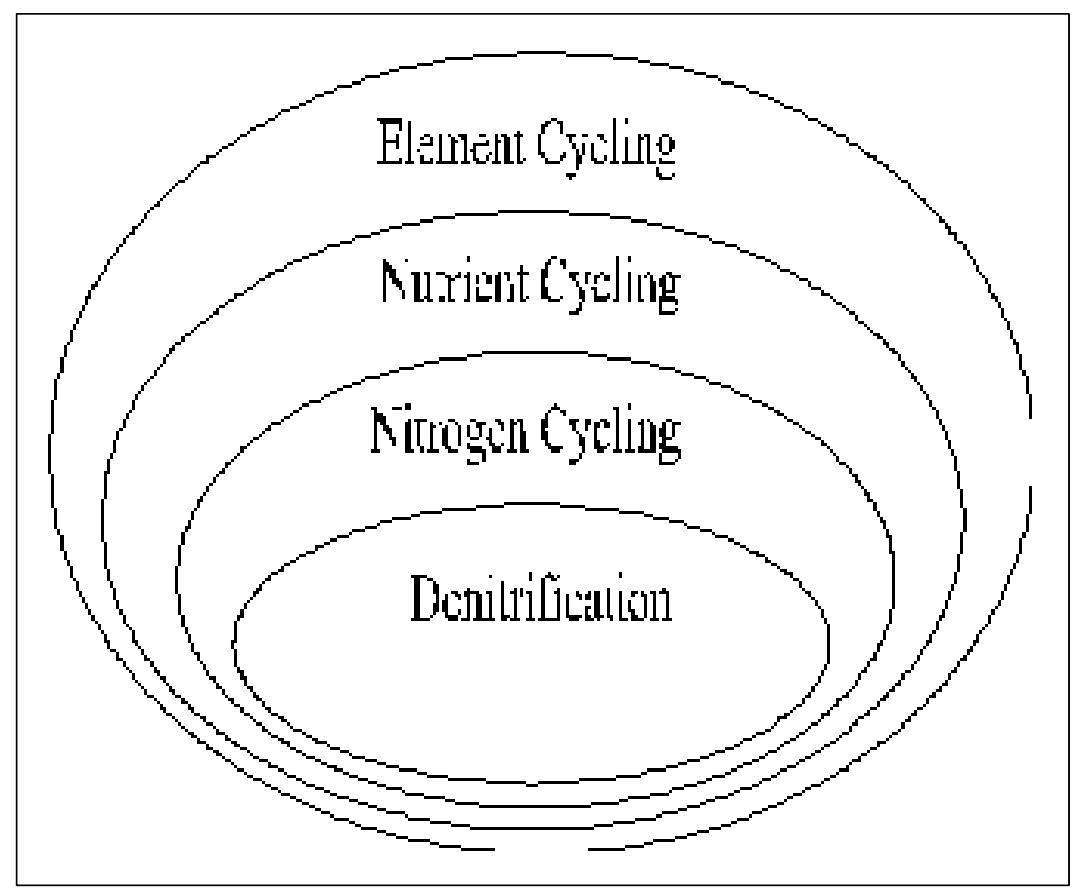

Figure 14. The hierarchy of wetland functions.

general function attributable to wetlands. Within the context of element cycling it is possible to define more specific functions such as nutrient cycling. Within the context of nutrient cycling, it possible to define more specific functions such as nitrogen cycling or phosphorus cycling. Within the context of nitrogen cycling it is possible to define more specific functions such as denitrification (i.e., transformation of nitrate to gaseous nitrous oxide and molecular nitrogen). A similar hierarchy of functions can be defined for other categories of functions such as habitat [e.g., provide habitat for wildlife in general, provide habitat for a group of species (e.g., amphibians, mammals, neotropical migrant birds), and finally provide habitat for a species (e.g., Cerulean warblers, tiger salamanders)].

In deciding which level of the hierarchy functions to select, the assessment team should consider the advantages and disadvantages inherent at each level of the hierarchy. Developing assessment models for more general functions typically requires a larger number of model variables. In addition, at the more general level, the variability encompassed by each model variable tends to be greater. Consequently, assessment models with many model variables are often insensitive, requiring large changes in the field measure to have a noticeable effect on the functional index. The degree of sensitivity also depends on the nature of the aggregation equation since multiplicative and minimum/maximum functions can exert a controlling 
influence on model output regardless of the number of model variables (see "Sensitivity Analysis" in Chapter 5). Another problem with selecting general functions is the difficulty of identifying an appropriate independent measure of function with which to validate the assessment model. This is discussed in greater detail in the next section.

Selecting functions at a high level of detail or specificity also has inherent problems. First there is the proliferation in the number of functions required to represent the range of characteristics and processes that occur in wetlands. Another potential problem is the lack of knowledge necessary to develop an accurate assessment model, and the difficulty in collecting field data that requires a high level of technical expertise. It should be noted that the functions selected for published guidebooks are in the midrange of the hierarchy of functions described above (Brinson et al. 1995, Ainslie et al. 1999, Noble et al. 2002, Rheinhardt et al. 2002, Hauer et al. 2002).

Another consideration that comes into play when selecting functions at a high level of specificity is the availability of time and resources for conducting functional assessment under 404 and similar situations. In order for the HGM Approach to be a practical tool in the context of 404, it must be possible to complete the required field work for the assessment in one day or less. Certain factors such as a large or heterogeneous permit area may increase this time frame; however, experience has shown that at a typical site it is possible for trained personnel to collect the field data necessary to run assessment models for four to eight functions with eight to fifteen model variables (Berkowitz et al. 2011). This represents a good rule of thumb when selecting functions for use in 404 or similar assessment scenarios.

\section{Define wetland functions}

The final consideration in the selection of wetland functions is the requirement to define them in a concise and quantifiable way. Unambiguous function definitions identify the variables that reflect the important attributes and processes influencing a function, and define the nature of the interaction between these variables. A concise definition consists of one or two sentences that clearly identify the ecosystem attributes and processes modeled. For example, the Low Gradient Riverine Regional Guidebook for Western Kentucky (Ainslie et al. 1999) defines the Temporary Storage of Surface Water function as, "The capacity of a riverine wetland to 
temporarily convey and store surface water during overbank flood events. The primary source of the surface water is normally an adjacent stream channel, but other sources can include overland flow, interflow, or direct precipitation." This definition provides clues to the attributes and processes that need to be captured as variables, including factors that affect the storage capacity of the wetland and the rate at which water passes through the wetland.

It is not uncommon for a function to be named or described in a general or ambiguous way, thereby making it difficult to define. For example, it would be difficult to concisely define a wetland function called "Maintain Characteristic Wildlife Community." This is because it is difficult to determine exactly what is meant by the term "wildlife community," and how deviations from "characteristic" wildlife community could be recognized and measured. There are several ways to recast this function so that it can be concisely defined and quantified. First, the assessment team may decide that the diversity of vertebrates in the wetland is a good indicator of the characteristic wildlife community. Based on this assumption, they could rename the function "Maintain the Diversity of the Vertebrate Community," and define it as the capacity of the wetland to maintain the characteristic diversity of endemic vertebrates. Alternatively, the assessment team may decide that in order to capture the multiple components implicit in the "Maintain Characteristic Wildlife Community" function, can be recast into several functions that explicitly deal with the multiple components. This might include functions such as "Diversity of Breeding Bird Species," "Density of Breeding Amphibians," and "Diversity of Mammals" for which a concise definition and quantitative measure can be identified.

In addition to being able to concisely define a function, it must be possible to identify an independent measure of each function along with appropriate quantitative units. For example, the Low Gradient Riverine Regional Guidebook for Western Kentucky (Ainslie et al. 1999) identifies the independent, quantitative measure of the Temporary Storage of Surface Water function as the volume of water stored by a wetland over a specified time period (i.e., $\mathrm{m} 3 / \mathrm{ha} / \mathrm{yr}$ ). Identification of an independent, quantitative measure of function is mandatory if assessment models are to be amenable to validation and accepted by the scientific and regulatory communities. The output (i.e., the FCI) from assessment models is validated by comparing it to an independent measure of function such as direct count of breeding bird species, or direct measure of sediment accretion. Such comparisons 
evaluate model accuracy, and provide information to revise the assessment model and improve its performance (see Chapter 7). Table 6 provides examples of quantitative measures for defining a variety of potential wetland functions.

Table 6. Wetland functions and potential independent measures of function.

\begin{tabular}{|c|c|}
\hline Hydrologic Functions & Potential Metrics \\
\hline $\begin{array}{l}\text { Temporary Storage of Surface Water } \\
\text { Subsurface Storage of Water } \\
\text { Maintenance of Base Flows }\end{array}$ & $\begin{array}{l}\text { Average volume of water stored (m3/ha/yr) } \\
\text { Percent reduction in flow peaks }(\%) \\
\text { Lag time of input and output flow peaks (d) } \\
\text { Percent reduction in water velocity }(\%) \\
\text { Volume of water absorbed }\left(\mathrm{m}^{3} / \mathrm{ha} / \mathrm{yr}\right) \\
\text { Volume of available pore space }\left(\mathrm{m}^{3} / \mathrm{ha}\right) \\
\text { Difference in low flows above/below wetland (\%) } \\
\left.\text { Wetland contribution to low flows ( } \mathrm{m}^{3} / \mathrm{ha} / \mathrm{d}\right)\end{array}$ \\
\hline Biogeochemical Functions & Potential Metrics \\
\hline $\begin{array}{l}\text { Cycling of Nutrients } \\
\text { Removal of Elements and Compounds } \\
\text { Retention of Particulates } \\
\text { Export of Organic Carbon }\end{array}$ & $\begin{array}{l}\text { Net primary productivity }(\mathrm{kg} / \mathrm{ha} / \mathrm{yr}) \\
\text { Annual turnover of detritus }(\mathrm{kg} / \mathrm{ha} / \mathrm{yr}) \\
\text { Denitrification rate }(\mathrm{kg} / \mathrm{ha} / \mathrm{yr}) \\
\text { Soil denitrification enzyme activity }(\mathrm{DEA}, \mathrm{gN} / \mathrm{g} / \mathrm{d}) \\
\text { Amount of sediment trapped (tons/ha/yr) } \\
\text { Sediment accretion rate }(\mathrm{cm} / \mathrm{yr}) \\
\text { Rate of biomass export }(\mathrm{kg} / \mathrm{ha} / \mathrm{yr})\end{array}$ \\
\hline Habitat Functions & Potential Metrics \\
\hline $\begin{array}{l}\text { Support Characteristic Plant Community } \\
\text { Support Characteristic Wildlife Community } \\
\text { Support Characteristic Invertebrate Community } \\
\text { Support Landscape/Regional Biodiversity }\end{array}$ & $\begin{array}{l}\text { Diversity of native plant species (index, } \mathrm{H}^{\prime} \text { ) } \\
\text { Number of rare or endemic species (count) } \\
\text { Species richness of vertebrates (count) } \\
\text { Number of species of forest interior birds (count) } \\
\text { Density of breeding amphibians (number/ha) } \\
\text { Invertebrate biomass (kg/ha) } \\
\text { Arthropod species richness (count) } \\
\text { Number of species unique or rare in the region (count) } \\
\text { Number of food web links (count) }\end{array}$ \\
\hline
\end{tabular}

\section{Conceptualize assessment models}

Once the assessment team has selected and defined a suite of functions for the regional wetland subclass, the next task is to conceptualize an assessment model for each function. Conceptualizing assessment models requires that the assessment team: 1) select and define variables to represent the structural components and processes of the wetland ecosystem and the surrounding landscape that significantly influence functional capacity, 2) select and define the metrics that will be used to measure the variables, 
3) define the relationship between variables and functional capacity, and 4) construct an equation for deriving the FCI. The assessment model resulting from the conceptualization process represents an assessment model based on the knowledge and experience of experts, the literature, and existing data. Remember that the conceptual assessment model only represents the first phase in the development of the assessment model. The second and third phases of development involve further testing, calibration, and validation of the conceptual assessment models.

\section{Select and define variables}

The first step in the conceptualization process is to identify the attributes, structural components, and processes of the wetland and surrounding landscape that have a significant influence on how a function is performed by the regional wetland subclass. This is when a concise definition for each wetland function pays off. There are several good sources of information that can be used to identify potential variables, including several reports that summarize information on the physical, chemical, and biological variables used in a wide variety of assessment methods (Canter and Hill 1979, Adamus and Brandt 1990, Adamus 1992, Simenstad et al. 1991, Solomon and Sexton 1994). Potential variables can also be identified using the literature dealing with more quantitative approaches to assessing hydrologic and biogeochemical functions (Brunner 1988; Chescheir et al. 1987, 1984; Faulkner et al. 1989; Guertin et al. 1987; Gunderson 1989; Hammer and Kadlec 1986; Heliotus and DeWitt 1987; Kadlec 1988; LaBaugh 1986; Rosenberry 1990; Tiedje et al. 1981; Tiedje 1982; Welcomme 1979; Winter 1981).

Published regional guidebooks are another potentially valuable source of information for identifying variables. However, before adopting a variable from another source, the assessment team should critically analyze the rationale provided for a particular variable to determine if it is relevant and appropriate in the context of the regional wetland subclass under consideration. In some cases, specific variables, or an entire assessment model, can be adopted, particularly when both the existing model and the model being developed are for similar wetland classes. For example, some of the variables and assessment models for low gradient riverine systems developed for Western Kentucky (Ainslie et al. 1999) might be adopted for assessing low-gradient riverine systems throughout the Lower Mississippi River Valley and the coastal plain. Rarely, however, will variables or assessment models translate as well between regional wetland subclasses 
that belong to different classes (e.g., riverine wetlands versus depressional wetlands).

The variables gathered from published assessment models and other literature sources should also be expanded through the process of brainstorming about potential additional structural components and processes that are important for a particular regional wetland subclass. The objective is to make sure that all the important factors have been identified, not necessarily how they interact. For example, if the function under consideration is related to habitat, identify all of the factors that are critical to short-term survival and long-term reproductive success of the plants or animals under consideration. This might include the presence of certain plant species or other specific habitat features such as snags, mature trees, and seasonal pools. Similarly, if the function is related to hydrology, identify the factors that affect how water gets to the wetland, moves through the wetland, and leaves the wetland. Remember, not all of the variables identified during the initial literature search and brainstorming period will necessarily end up in the final assessment model.

After identifying potential variables, the assessment team should critically review the list for redundant, irrelevant, and insensitive variables. The majority of assessment models developed for the HGM Approach to date include two and six variables. This number represents a good rule of thumb, but it is not a rigid requirement. As indicated above, functions at a higher level of detail (i.e., lower in the hierarchy of functions) usually require fewer variables, while more general functions will require a greater number of variables. If the number of variables required exceeds six or seven, consider defining two or more specific functions instead.

Another important factor to consider is the range of values exhibited by a variable and the sensitivity of variables to potential impacts. Wetlands are dynamic systems subject to change on a range of spatial and temporal (e.g., daily, seasonal, and annual basis) scales. Variables that exhibit a wide range of values under minimally disturbed conditions may not be useful for detecting change resulting from natural processes or disturbance. Remember that in the context of 404 and similar assessment applications, the primary use of the models is to detect changes in functional capacity that result from project impacts. The types of impacts associated with 404 typically include dredging, filling, levee construction, land clearing, draining, ditching, and other actions that alter hydrologic regimes. These 
impacts are not subtle, and assessment models should be capable of detecting the changes that are likely to occur as a result of these impacts. Variables that are insensitive to the impacts that typically occur in a regional wetland subclass are of little use in detecting change. Here again, the knowledge and field experience of the assessment team are essential. Finally, remember that the variables selected must be measurable, either directly or indirectly, using field or remote metrics. Variables that are difficult or impractical to measure should be discarded.

Once variables have been selected, they need to be defined. As with functions, variables need to be defined in a concise manner. For example, the Low Gradient Riverine Regional Guidebook for western Kentucky (Ainslie et al. 1999) defines the variable "gradient" as, "...the slope of the floodplain in a direction parallel to the flow of floodwater." Once the model variable has been defined, the criteria and rationale used in selecting model variables should be explained, including the specific structural component or processes that each variable represents and how it influences the functional capacity. Documentation should also provide literature references and personal experiences of assessment team members to support this discussion, as well as a clear explanation of assumptions and identification of potential data gaps.

\section{Select and define metrics}

The next task is to determine the metric that will be used to measure each variable. Implicit in this decision is selecting the scale of measurement that will be used. There are four basic scales of measurement: nominal, ordinal, interval, and ratio (Zar 1999). The nominal scale is a qualitative scale of measure in which the variable being measured is assigned to one of two or more mutually exclusive and unrelated categories. For example, a variable may be assigned to the present or absent category, or a soil might be assigned to a clay, loam, or sand category. Equivalence (e.g., two soils are in the same category) is the only mathematical relation or operation applied to nominal scale data (Table 7 ).

The ordinal scale is also a qualitative scale of measurement in which three or more categories are ranked or ordered in relation to each other, and the numerical distance between categories is unknown. The Braun-Blanquet scale for measuring plant abundances (e.g., o-5, 5-10,10-25, 25-50, etc.) is an example of an ordinal scale. Equivalence and order are the mathematical relations and operations applied to ordinal scale data. 
Table 7. Permissible mathematical relations and operations for various scales of measurement.

\begin{tabular}{|l|l|l|l|l|}
\hline \multirow{2}{*}{$\begin{array}{l}\text { Scale of } \\
\text { Measurement }\end{array}$} & \multicolumn{4}{|c|}{ Permissible Mathematical Relations or Operations } \\
\cline { 2 - 5 } & Equivalence & Order & $\begin{array}{l}\text { Addition and } \\
\text { Subtraction }\end{array}$ & $\begin{array}{l}\text { Multiplication and } \\
\text { Division }\end{array}$ \\
\hline Nominal & $*$ & & & \\
\hline Ordinal & $*$ & $*$ & & $*$ \\
\hline Interval & $*$ & $*$ & $*$ & $*$ \\
\hline Ratio & $*$ & $*$ & $*$ & \\
\hline
\end{tabular}

The interval and ratio scales are quantitative measures in which the numerical distance between categories is specified and constant. In the case of the interval scale, the origin or zero point is defined arbitrarily, whereas in the case of the ratio scale, it represents a true origin. Consequently, all measurements are made in terms of real numbers. An example of the interval scale is the Fahrenheit temperature scale in which the change in temperature between 40 and 41 and 50 and 51 degrees is the same, but the zero point is arbitrary. Examples of the ratio scale include weight of an organism or the size of a tree in terms of diameter at breast height (dbh). The interval between units is constant, and the zero point has true meaning (i.e., no weight and no diameter). Permissible mathematical relations and operations for the interval and ratio scales of measurement include equivalence, order, addition, subtraction, multiplication, and division.

For many variables, standardized sampling methods and metrics already exist (Chapter 5). Whenever possible, standard methods and metrics are preferred because field data collectors will often be familiar with them. For example, tree basal area can be measured using a plot or plotless sampling method and a metric of $\mathrm{m}^{2} / \mathrm{ha}$. Similarly, frequency of flooding might be measured using a regional curve and the metric of return interval in years. Deciding which sampling method and scale of measurement is appropriate for collecting information about a model variable depends on several factors. First, given similar time and effort requirements, one should usually select a quantitative measurement rather than a qualitative one. Quantitative data can be transformed to a qualitative scale of measurement at a later time if deemed appropriate, whereas the opposite is not true. Furthermore, models with quantitatively measured variables are more amenable to calibration and validation. Many of the variables typically included in assessment models can be readily sampled in the field using a quantitative scale of measurement. For example, many field personnel are familiar with 
plot, plotless, or transect sampling methods and can easily collect vegetation data, such as basal area for woody plants and percent cover for herbaceous plants, on a quantitative scale. As an aside, whenever possible, consider using data collection methods that correspond to the methods already being used to collect data for the purposes of wetland delineation (Environmental Laboratory 1987)

Secondly, the practical constraints of time, personnel, and technical expertise limit the number of and type of variables that can be sampled. For some variables it is unlikely that a quantitative scale of measurement will be possible for technical reasons, such as long or extensive sampling periods or the need for specialized equipment. For example, it is difficult to get a measure of average $\mathrm{pH}$, water temperature, depth of flooding, or depth to water table because of the need for repeated samples over a long period. The rule of thumb mentioned earlier still applies. In a typical permit review situation, there is usually one day (and at most several days) available to collect field data. Any requirement to collect information over longer periods, or with highly specialized equipment, may result in data not being collected and the affected functions not being assessed.

This step is completed by documenting the criteria and rationale used to select the sampling method and measurement scale for each model variable.

\section{Define the relationship between variables and functional capacity}

As indicated, metrics are collected using different units and scales of measurement and must be transformed into a set of comparable, unitless measures (Schuster and Zuuring 1986, Smith and Theberge 1986) before they can be combined in an equation to produce an FCI. In the HGM Approach, transformed metrics are called subindices and are assigned a value from 0.0 to 1.0. The transformation is based on what has variously been called a value function (O'Banion 1980), scalar (Westman 1985), or a "normalization and standardization" procedure (Barbour et al. 1995).

The relationship between a metric and functional capacity, as expressed by the subindex, is initially defined by the assessment team based on their knowledge and experience, the literature, and available data. This relationship will subsequently be subject to review and revision after data from reference wetlands have been analyzed and the assessment models undergo calibration, verification, and validation. 
In defining this relationship between metric values and subindices, two factors must be considered. The first is that, by definition, the metric values exhibited in reference standard wetlands receive a subindex of 1.o. This is because reference standard wetlands represent minimally disturbed wetland in minimally disturbed landscapes, and perform the suite of all functions ascribed to the regional wetland subclass at the highest sustainable level. Second, as metric values deviate from the metric values exhibited in reference standard wetlands, the subindex assigned to a metric decreases to reflect the decrease in functional capacity.

The relationship between a metric and functional capacity (i.e., subindex) can take on several forms. For example, in Figure 15 the solid line indicates that the relationship between tree basal area metric values and functional capacity decreases linearly and continuously from a value of 1.0 when the metric value is greater than $30 \mathrm{~m}^{2} /$ ha (i.e., value exhibited by reference standard wetlands). In Figure 16 the soil type metric values are sampled using the nominal scale categories of organic, clayey, loamy, and sandy and the subindex is assigned to each discrete category. As in the case of the tree basal metric, the subindex of 1.0 reflects the metric value exhibited in reference standard wetlands, and a lower subindex is assigned to other soil types as they deflect from these reference standard conditions.

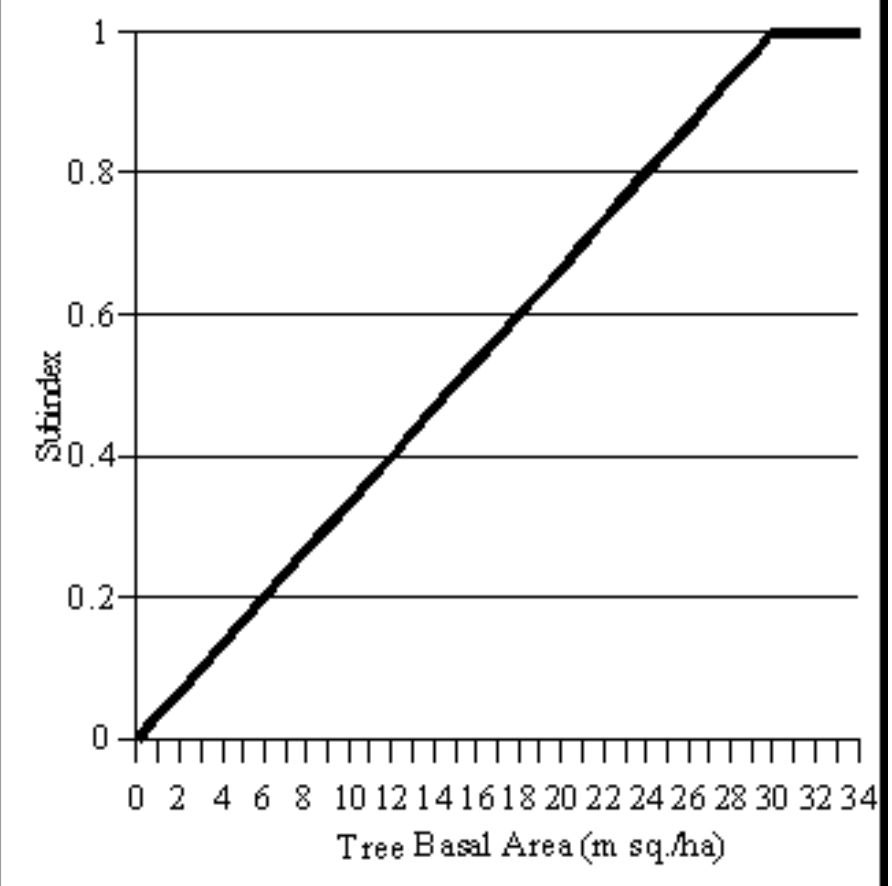

Figure 15. Relationship between tree basal area metric and functional capacity expressed as a subindex. 


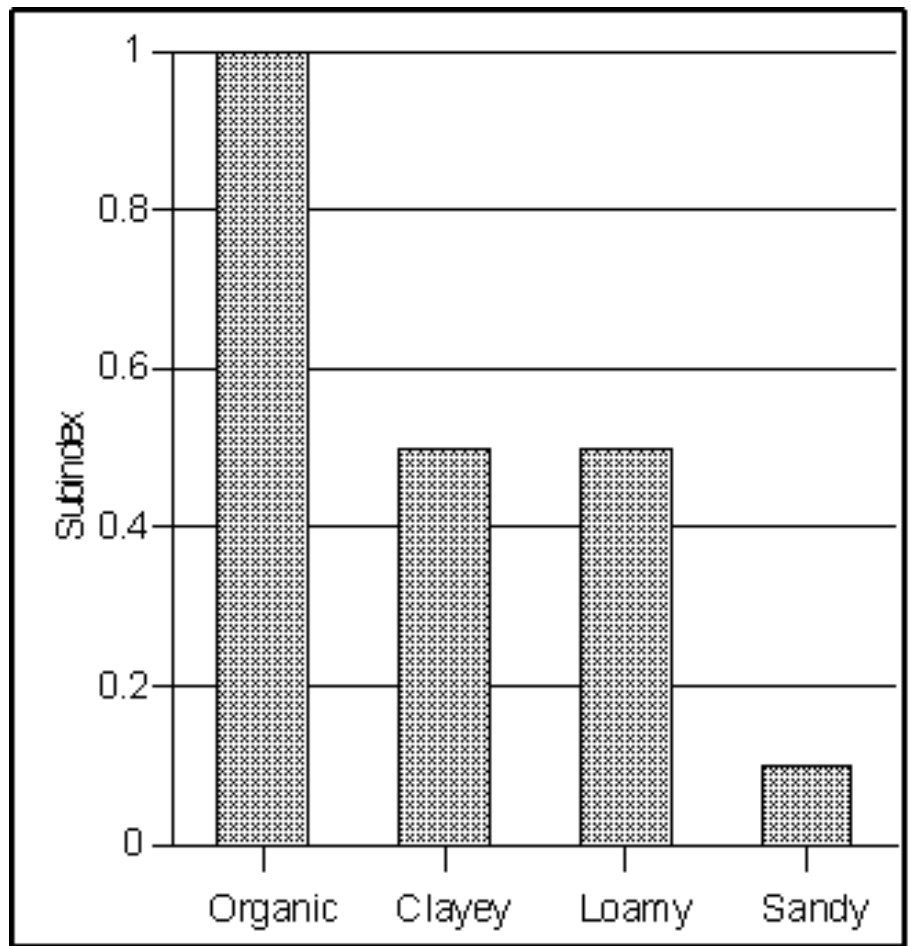

Figure 16. Relationship between the soil type metric and functional capacity expressed as a subindex.

It is impossible for the assessment team to finalize the relationship between metric values and functional capacity during the conceptualization of the assessment model. This can only be done following the collection and analysis of reference data during model calibration (Chapter 6). It is important, however, that the assessment team define a preliminary relationship between metrics and functional capacity using their knowledge and experience with the regional wetland subclass.

\section{Define the relationship between variables}

The final step in conceptualizing the assessment model is to develop a mathematical equation for combining model variables and deriving the FCI. The objective is to capture how variables interact to influence functional capacity. For example, the Temporary Storage of Surface Water function in the Low Gradient Riverine Regional Guidebook for western Kentucky (Ainslie et al. 1999) uses the following variables:

$$
\begin{aligned}
\mathrm{V}_{\text {FREQ }} & =\text { frequency of flooding } \\
\mathrm{V}_{\mathrm{XSEC}} & =\text { cross-sectional area } \\
\mathrm{V}_{\text {ROUGH }} & =\text { roughness coefficient } \\
\mathrm{V}_{\text {GRADIENT }} & =\text { slope }
\end{aligned}
$$


These variables are aggregated to produce an FCI using the following equation: $\mathrm{FCI}=\left[\left(\mathrm{V}_{\mathrm{FREQ}} \mathrm{x} \mathrm{V}_{\mathrm{XSEC}}\right)^{1 / 2} \mathrm{x}\left(\mathrm{V}_{\mathrm{ROUGH}}+\mathrm{V}_{\mathrm{GRADIENT}}\right) / 2\right]^{1 / 2}$

Variables can interact in many ways. However, the equations used in the HGM Approach are relatively simple and a few basic types of relationships are usually adequate. For example, if all of the variables are of equal importance, the relationship between variables is additive. The mathematical function that describes this relationship is the mean of the subindices of each model variable. There are two methods of calculating an average value, the arithmetic mean: $\left(V_{1}+V_{2}+V_{3}\right) / 3$, and the geometric mean: $\left(V_{1} * V_{2} * V_{3}\right)^{1 / 3}$. The arithmetic mean results in a larger average because it is relatively insensitive to lower value subindices, and is therefore a good choice when variables in a group have a somewhat compensatory relationship. The geometric mean should be used when the compensatory relationship between variables is weak or lacking. For example, in the Temporary Storage of Surface Water function above, both frequency of flooding and cross-sectional area have no compensatory effect. A significant change in either of these variables indicates that water cannot get to the wetland. If water cannot get to the wetland, surface water is not stored in the wetland.

Multiplication of the mean of a group of variables by another variable (i.e. $((\mathrm{V} 1+\mathrm{V} 2) / 2) * \mathrm{~V} 3)$ can create a switch effect: if $\mathrm{V} 3=0$, then the function capacity index equals $\mathrm{o}$, but if $\mathrm{V} 3>0$, then the function is the mean of $\mathrm{V} 1$ and V2 weighted by V. Switch indices are often used in situations where a variable (e.g., flooding or drainage) serves to turn a function on or off regardless of the condition of other variables.

Another way to increase or decrease the influence of a variable in the FCI is to modify its weight in the equation. This is done by adjusting its coefficient (e.g., in a sum or arithmetic mean) or its exponent (e.g., in a geometric mean). For example, consider the following simple aggregation equation: $\mathrm{FCI}=\left(\mathrm{V}_{\mathrm{A}}+\mathrm{V}_{\mathrm{B}}+\mathrm{V}_{\mathrm{C}}\right) /$ 3. To increase the influence of $\mathrm{V}_{\mathrm{A}}$ in the calculation of FCI, one could increase its coefficient to 2, remembering to increase the divisor to a value of 4 so that FCI does not exceed 1 . Thus the equation becomes $\mathrm{FCI}=\left(2 \mathrm{~V}_{\mathrm{A}}+\mathrm{V}_{\mathrm{B}}+\mathrm{V}_{\mathrm{C}}\right) / 4$ (2). For a geometric mean, the equivalent procedure is to increase a variable's exponent. For example, the influence of $\mathrm{V}_{\mathrm{A}}$ in the equation $\mathrm{FCI}=\left(\mathrm{V}_{\mathrm{A}} \times \mathrm{V}_{\mathrm{B}} \times \mathrm{V}_{\mathrm{C}}\right)^{1 / 3}$ (3) can be increased by squaring $\mathrm{V}_{\mathrm{A}}$. The modified equation becomes $\mathrm{FCI}=\left(\mathrm{V}_{\mathrm{A}}^{2} \times \mathrm{V}_{\mathrm{B}} \times \mathrm{V}_{\mathrm{C}}\right)^{1 / 4}$ (4). 
U.S Fish and Wildlife Service (USFWS) (1980a, 1980b, 1980 c, 1981) publications discuss several of these types of relationships including cumulative, limiting, compensatory, partially compensatory, and controlling (Table 8). Figure 17 shows the effect of these relationships on the FCI. Experience has shown that of these five types of relationships, the cumulative and limiting types are most important for the wetland functions being assessed using the HGM Approach.

Table 8. Types of interactions between model variables, and their mathematical expression, that may be useful in developing assessment models for the HGM Approach.

\begin{tabular}{|l|l|l|}
\hline Type of Interaction & Mathematical Operation & Example \\
\hline Cumulative & Addition & $\mathrm{FCl}=V_{A}+V_{B}+V_{C}$; if sum $>1.0$ then $\mathrm{FCl}=1.0$ \\
\hline Limiting & Minimum & $\mathrm{FCl}=\mathrm{MIN}\left(V_{A}, V_{B}\right)$ \\
\hline Fully compensatory & Maximum & $\mathrm{FCl}=\mathrm{MAX}\left(V_{A}, V_{B}\right)$ \\
\hline Partially compensatory & Arithmetic mean or average & $\mathrm{FCl}=\left(V_{A}+V_{B}+V_{C}\right) / 3$ \\
\cline { 2 - 3 } & Geometric mean & $\mathrm{FCl}=\left(V_{A} \times V_{B} \times V_{C}\right)^{1 / 3}$ \\
\hline Controlling & Product & $\mathrm{FCl}=V_{A} \times\left(V_{B}+V_{C}\right) / 2$ \\
\hline
\end{tabular}

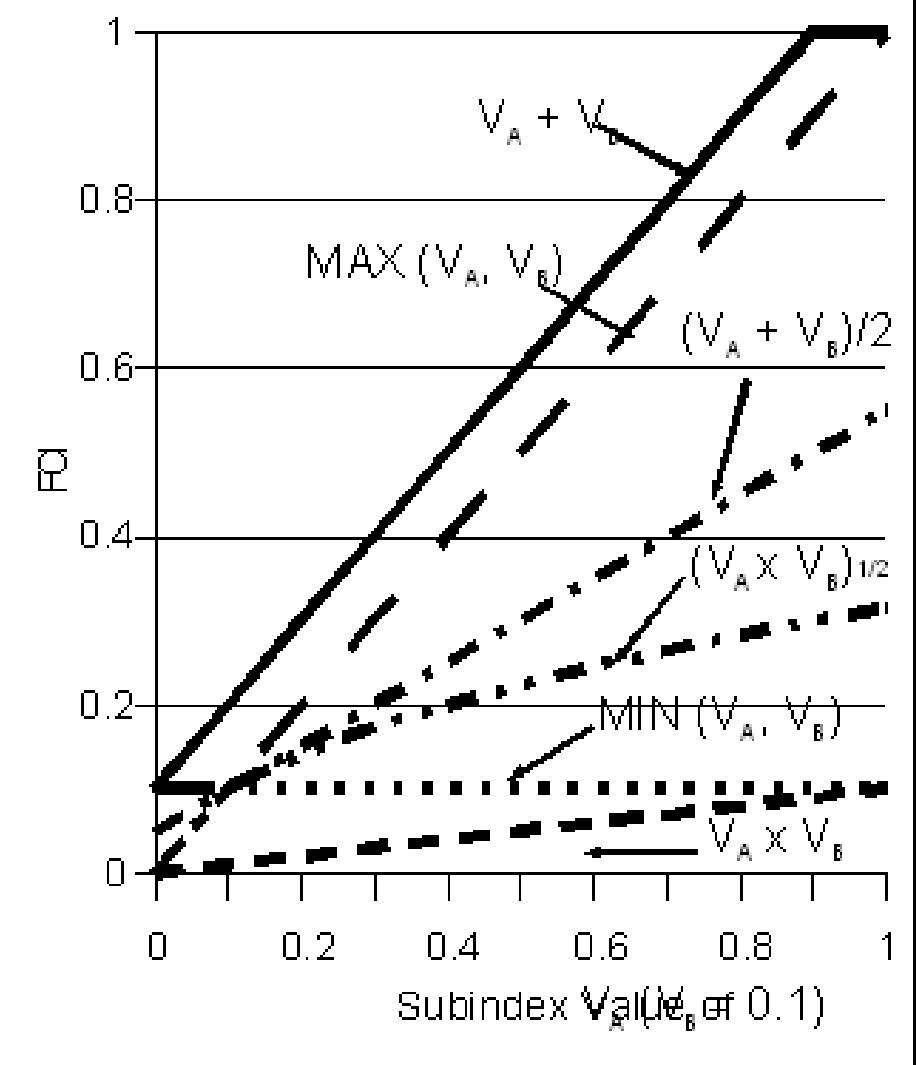

Figure 17. Effects of various mathematical operations in combining $V_{A}$ and $V_{B}$. In all cases, the value of $V_{B}$ is fixed at

0.1 , while $V_{A}$ varies between 0 and 1.0 . 


\section{Limiting relationship}

A limiting relationship occurs when a low value for any one variable overrides the effects of other variables; thus, the functional capacity index is equal to the lowest of the subindex values. The appropriate mathematical expression is a minimum (Table 8). For example, limiting relationships are often used in habitat models (USFWS 1981) to express the relative availability of two or more essential life requisites for a species, such as food, water, and nesting sites. Thus, overall habitat suitability is equal to the lowest of the three subindices, reflecting the life requisite that is in shortest supply.

\section{Cumulative relationship}

A cumulative relationship exists when variables complement each other, such that either variable alone, or both in combination, contributes to functional capacity. The appropriate mathematical expression is a sum, with the qualification that FCI cannot exceed 1.o (Table 8). For example, a hypothetical model for Particulate Retention might use a cumulative interaction if retention time of the water ( $\left.V_{\text {RETENTION }}\right)$ or roughness of the wetland surface $\left(\mathrm{V}_{\mathrm{ROUGH}}\right)$, or a combination of both variables, is sufficient to achieve optimal functional capacity (e.g., $\mathrm{FCI}=\mathrm{V}_{\mathrm{RETENTION}}+\mathrm{V}_{\mathrm{ROUGH}}$; if sum $\geq 1.0$ then FCI $=1.0$ ). Thus, optimal functional capacity is achieved whenever either variable equals 1.0 (even if the other variable is zero), or when their sum equals or exceeds 1.o. An FCI of zero only occurs when the subindices for both variables are zero.

\section{Compensatory relationship}

In a compensatory relationship, a high value for one variable compensates, either in full or in part, for a lower value of another variable. The interaction is fully compensatory if the final FCI is equal to the highest of the component subindices. In this case, the appropriate mathematical expression is a maximum (Table 8). A partially compensatory relationship exists when two or more variables contribute equally and independently to the level of function. Mathematical expressions used to model partially compensatory interactions include the arithmetic mean (or average) and the geometric mean. In each case, the resulting FCI is between the most extreme values of the subindices. The arithmetic mean is relatively less sensitive to subindices with low values. Therefore, when subindex values for the variables are different, the arithmetic mean returns a higher result 
than the geometric mean (Figure 17). An important difference between the two mathematical expressions is that the geometric mean returns a zero whenever any of the component subindices is zero, whereas the arithmetic mean returns a zero only when all of the subindices are zero.

\section{Controlling relationship}

A controlling relationship occurs when the presence of one environmental feature or process is critical to the performance of a function, and thus has the potential alone to control the function. The appropriate mathematical operation is a product (Table 8). For example, a simple model for Organic Carbon Export might contain the following aggregation equation: $\mathrm{FCI}=$ $\mathrm{V}_{\text {FREQ }} \times\left(\mathrm{V}_{\mathrm{LITTER}}+\mathrm{V}_{\mathrm{CWD}}\right) / 2$. Carbon export is affected by abundance leaf litter $\left(\mathrm{V}_{\text {LITTER }}\right)$ and coarse woody debris $\left(\mathrm{V}_{\mathrm{CWD}}\right)$, which are grouped and averaged because they contribute equally and independently to the availability of material for export. However, carbon export cannot occur unless floodwaters scour the site. Combining $\mathrm{V}_{\mathrm{FREQ}}$ by means of a product reduces FCI to zero if the site does not flood, despite high values of the other variables. 


\section{Reference Wetlands and Reference Wetland Data}

\section{Introduction}

The chapter begins by defining terms related to reference wetlands, and then discusses the purpose of reference wetlands, strategies for identifying and selecting reference wetlands, the number of reference wetlands required, designation of reference standard wetlands, and the use of historical information in reconstructing reference standard wetlands. This is followed by a discussion of the steps required to collect and manage reference wetland data.

\section{Reference wetland definitions}

Reference wetlands are specific wetland sites within a reference domain that represent the full range of conditions exhibited by wetlands in the wetland class (Table 9). The full range of conditions includes conditions that result from both natural processes and cycles (e.g., succession, channel migration, fire, erosion, and sedimentation) and cultural alteration (e.g., clear cutting, high grading, grazing, urban development, channelization, dredging, filling, snagging, levee building, etc.).

Table 9. Reference wetland terms and definitions.

\begin{tabular}{|l|l|}
\hline Terms & Definitions \\
\hline Reference Wetlands & $\begin{array}{l}\text { A group of wetlands that represent the full range of variability exhibited by wetlands } \\
\text { in a regional wetland subclass. The full range of variability includes variability } \\
\text { resulting from natural processes and cycles (e.g., succession, channel migration, } \\
\text { fire, erosion, and sedimentation) in addition to variability resulting from cultural } \\
\text { alteration or disturbance (e.g., clear cutting, high grading, grazing, urban } \\
\text { development, channelization, dredging, snagging, and levee building). }\end{array}$ \\
\hline $\begin{array}{l}\text { Reference Standard } \\
\text { Wetlands }\end{array}$ & $\begin{array}{l}\text { The subset of reference wetlands that represent the natural range of variability } \\
\text { exhibited by wetlands in the regional wetland subclass. The natural range of } \\
\text { variability exhibited by reference standard wetlands is used to establish reference } \\
\text { standard conditions for calibrating assessment model variables and functional } \\
\text { capacity indices. By definition, a functional capacity index of 1.0 is assigned to all } \\
\text { functions in reference standard wetlands. }\end{array}$ \\
\hline Reference Standard \\
Condition(s) & $\begin{array}{l}\text { The condition, or range of conditions, exhibited by a metric in reference standard } \\
\text { wetlands. By definition, a variable subindex of 1.0 is assigned to the reference } \\
\text { standard condition. }\end{array}$ \\
\hline Reference Domain & $\begin{array}{l}\text { The geographic area from which reference wetlands are selected to represent the } \\
\text { range of conditions exhibited in the regional wetland subclass. }\end{array}$ \\
\hline
\end{tabular}


Reference standard wetlands are the subset of reference wetlands used to establish the standard of comparison for calibrating variables and functional capacity indices (see Section in Chapter 3 titled "Overview of Assessment Models"). In the HGM Approach, reference standard wetlands represent the "minimally disturbed" condition, in the sense of Stoddard et al. (2006). This criterion is based on the assumption that minimally disturbed wetlands perform the highest, sustainable level of function across the suite of functions ascribed to the wetland subclass. Using minimally disturbed as the criteria for selecting reference standard wetlands also ensures compliance with the mandate of the Clean Water Act to maintain the physical, chemical, and biological integrity of wetlands and waters of the United States through the execution of the 404 Regulatory Program, and the national policy prescribing a "no net loss" of wetland function (Executive Order 11990).

The reference domain is the geographic area from which reference wetlands are selected to represent the wetland subclass (Table 9). The reference domain is relatively homogenous with respect to the factors that influence how wetlands in a wetland subclass function. Chapter 2 provides a discussion on approaches for selecting the reference domain.

\section{Purpose of reference wetlands}

Reference wetlands serve several purposes in the HGM Approach. First, they provide a physical representation of wetlands in the subclass that can be observed and sampled repeatedly. Second, reference wetlands make it possible to establish the range of conditions exhibited by metrics, making it possible to calibrate variables and functional capacity indices (FCI). Third, reference wetlands provide a template for developing design specifications for wetland restoration based on reference standard conditions.

\section{Select reference wetlands}

\section{Inventory and screening}

The first step in selecting reference wetlands is to conduct an inventory of the wetlands belonging to the wetland subclass in the reference domain. This inventory can draw from existing reports, maps, and geospatial data including national, state, regional, and local wetland or aquatic resource inventories, wetland regulatory permit databases, mitigation bank databases, advanced identification studies, special area management 
plans, habitat conservation plans, watershed management plans, and individuals with broad knowledge of wetlands in the region. Persistence and creativity are key in pursuing these sources of information, as they may not be widely known or distributed.

Once the inventory is complete, the next step is to conduct field reconnaissance to screen potential candidate reference wetland sites. The objective is to identify sites that represent the full range of conditions that exist in the reference domain from highly disturbed sites in highly disturbed landscapes to minimally disturbed sites in minimally disturbed landscapes. It is also important, in the context of minimally disturbed sites, to select a range of sites that reflect the various types of natural disturbances and cycles that occur in the reference domain. A form similar to the one shown in Table 10 will help to ensure that all appropriate information is collected and organized efficiently. Displaying reference wetland locations on maps at a scale of 1:100,000 will help to ensure that reference wetlands are identified throughout the reference domain.

Table 10. Reference wetland inventory form.

\begin{tabular}{|l|l|}
\hline \multicolumn{2}{|c|}{ Reference Wetland Summary Sheet } \\
\hline Site Name & Possum Creek Slough \\
\hline Site Number / Code & RB-123 \\
\hline Site Location Description & 5 miles past the Jitney Jungle on Highway 61 \\
\hline USGS 7.5 Minute Quad & Sharkey \\
\hline County Soil Survey & Sharkey - Sheet \#6 \\
\hline Township / Section / Range & Township 7W, Range 6N, NW 1/4, of NW 1/4, of Section 5 \\
\hline UTM Coordinates & 100798 3457586 \\
\hline HGM Class & Riverine \\
\hline Local Point of Contact & name (caretaker) phone\# \\
\hline Regional Subclass & Isolated depression \\
\hline Environment of Deposition & Historical backswamp of the Mississippi River \\
\hline Condition Class (1-5, 1=RS) & 1 - Primo reference standard site as good as it gets \\
\hline Type of Alteration & No hydrologic or land surface alterations \\
\hline Site Description & This site supports a mature stand of Quercus lyrata. It has... \\
\hline
\end{tabular}

\section{Number of reference wetlands}

A variety of factors will influence how many reference wetlands should be included in the reference wetland system. The first factor is, of course, the 
size and heterogeneity of the reference domain. Large reference domains will require more reference wetland sites to ensure adequate representation. Reference domains with a wide variety of alteration scenarios will require more reference wetland sites than reference domains where one or a few alteration scenarios exist. Another factor is the level of resolution necessary to detect the types of impacts that typically affect wetlands in the subclass. Finally, as in all projects, the ideal number of reference wetland sites dictated by the foregoing considerations must be balanced against the realities of budgets, time, and personnel.

A relatively simple way to determine when an adequate number of reference wetland sites have been sampled is to plot a measure of variability (e.g., variance or standard deviation) of variable measures as data from reference wetland sites are acquired (Figure 18). This approach is similar to a species area curve (Arrhenius 1921, Cain 1938, Condit et al. 1996). Statistical methods for determining what constitutes an adequate number of reference sites are discussed by Hughes and Noss (1992), Loftis et al. (1989), Walters et al. (1988), and Green (1979).

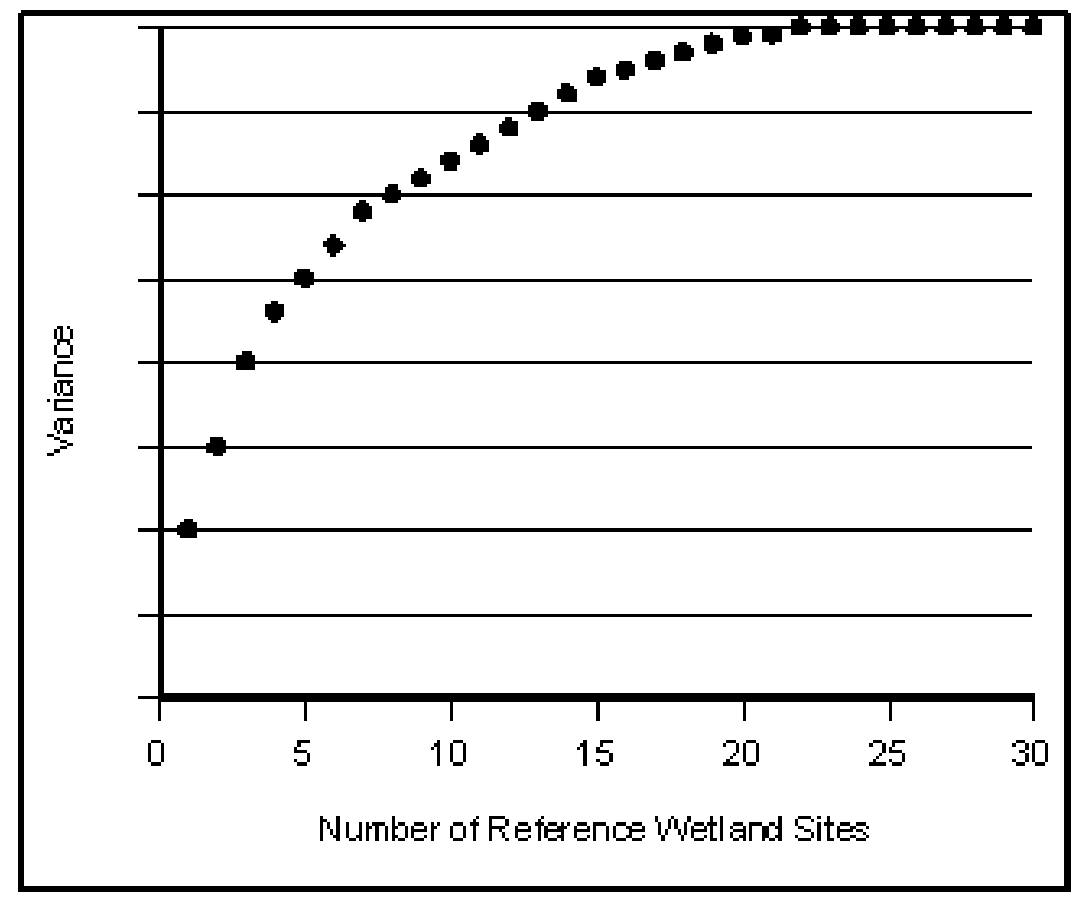

Figure 18. Trend in variance of a metric.

\section{Designating reference standard wetlands}

As indicated above, in the HGM Approach reference standard wetlands are the least-altered wetlands in the least-altered landscapes. This is based on 
the ecosystem focus of the HGM Approach and the assumption that these wetlands sustain the highest level of functioning across the suite of functions that are inherent to the wetland subclass. Several studies have outlined the use of this approach to designating reference standard wetlands (Brinson and Rheinhardt 1996, Rheinhardt et al. 1997).

\section{Prepare to collect reference wetland data}

Collecting and managing reference wetland data is a time-consuming and resource-intensive process. A number of preparatory tasks have proven to be helpful prior to actually going to the field and collecting reference wetland data. This chapter divides the preparatory tasks into the following three steps (example blank forms can be found in Figures 24 and 25):

- Step 1: Develop a reference wetland documentation form.

- Step 2: Develop an assessment variable documentation form.

- Step 3: Develop a field sampling protocol and field data form.

These steps and the subsequent tasks of collecting wetland reference data (Section titled "Collect Reference Wetland Data") and managing of wetland reference data (Section titled "Manage Reference Wetland Data") follow a logical sequence of implementation. However, it may be possible and useful in certain situations to conduct certain tasks out of the prescribed sequence. For example, developing the spreadsheets or database used to manage reference wetland data (Section titled "Manage Reference Wetland Data") could be initiated after Steps 1, 2, and 3 identified above, and before the collection of reference wetland data (Section titled "Collect Reference Wetland Data"). The three steps and subsequent tasks are also iterative in the sense that the results from some tasks may need to be revised as problems, insights, or new information are identified while conducting later tasks. Also, consider conducting a pilot study designed to proactively identify needs, problems, and insights that are likely to arise during a fullscale implementation.

\section{Variable documentation}

The second step in preparation for the collection of wetland reference data is to develop a method for documenting each of the variables used in assessment models. Typically this will include forms for documenting office and field measures for each variable as well as site information. This represents a formalization of the variable identification process initiated during the 
conceptualization of the assessment models (Section titled "Select and Define Variables"). Documentation of the variables serves as a reference and organizational tool for the development of the field sampling protocol and field data forms (Step 3 above), and as an archival appendix in the guidebook.

The specific format and content of the variable documentation should be determined by the assessment team. Information that the assessment team may find useful includes the following:

- Name, code, metric, and units.

- Equipment and supplies required to sample each variable.

- Procedure for sampling each variable, including step-by-step instructions, plot or transect schematics, and a method for determining the adequacy of the sample size.

- Graph showing the relationship between the metric value and the subindex.

- Aerial and site photographs.

Figure 20 is an example of a completed variable documentation form. In the example, the variable name and code constitute the header on each page, and the metric and its units are defined in the first section. The equipment necessary to measure the metric is listed in the second section. In the third section, the sampling procedure is laid out in a step-by-step manner. There are often various ways to sample a specific metric. For example, vegetation metrics can be sampled using plots, transects, or optical methods (Bonham 1989, Kent and Coker 1992, Brower et al. 1997). In the example form, a circular 0.4-ha plot layout is used, one of many possible methods to sample forest trees. The objective is to select a sampling procedure that can be applied reliably, while achieving appropriate levels of accuracy and precision given the nature of the metric.

Various statistical methods are available for determining how many samples are required to estimate the population mean of an assessment variable metric with the desired level of confidence (Bonham 1989, Brower et al. 1997, Zar 1996). However, more simplified methods that are suitable for determining the adequacy of sample size in the field include the speciesarea curve and performance curve methods (Cain 1938, Brower et al. 1997). The species area curve is commonly employed in vegetation sampling by graphing the number of plots or transects, or the size of the area sampled, on the " $x$ " axis, and the cumulative number of plant species on the " $y$ " axis 
(Figure 21). As the area sampled increases, the cumulative number of plant species will also increase up to a point at which the slope of the line begins to level off. The "break" in the slope of the line indicates that the areas sampled adequately represent the variability of plant species at the site.

\begin{tabular}{|c|c|c|}
\hline \multicolumn{3}{|c|}{ Reference Wetland Site Documentation: Six Mile Bayou Page 1 of 2} \\
\hline Reference Wetland Name: Six Mile Bayou & & Code: $\mathrm{RW}-015$ \\
\hline \multicolumn{3}{|c|}{ Regional Wetland Class / Subclass: Riverine / Backwater } \\
\hline Nearest City / Town: Kinlock, MS & \multicolumn{2}{|c|}{ State / County: Mississippi / Sunflower } \\
\hline \multicolumn{3}{|c|}{ GPS coordinates: $33^{\circ} 19^{\prime} 25.5^{\prime \prime} \mathrm{N} 90^{\circ} 43^{\prime} 27.5^{\prime \prime} \mathrm{W}$} \\
\hline \multicolumn{3}{|c|}{ Land Owner: name, 295 Kinlock Road, Kinlock, MS, phone\# } \\
\hline \multicolumn{3}{|c|}{$\begin{array}{l}\text { Directions: At the junction of Waco Road and Kinlock Road in Kinlock, MS proceed north on } \\
\text { Kinlock Road for } 1.6 \text { miles. At the junction of Kinlock Road and Bland Road turn left (west) and } \\
\text { proceed } 0.5 \text { miles. At the junction with Six Mile Bayou road follow the creek on the south side } \\
\text { of the road for a distance of approximately } 0.2 \text { miles to the northern boundary of the reference } \\
\text { wetland. Access is also possible via farm roads off Eakin Road south of the reference wetland. If } \\
\text { you choose this route, keys for locked gates can be obtained from the land owner Bob James. }\end{array}$} \\
\hline \multicolumn{3}{|l|}{ Description: } \\
\hline \multicolumn{3}{|c|}{$\begin{array}{l}\text { Geomorphology: The reference wetland is an abandoned meander of the Mississippi River in a } \\
\text { backswamp setting in the northeastern quarter of Six Mile Bayou. }\end{array}$} \\
\hline \multicolumn{3}{|c|}{$\begin{array}{l}\text { Vegetation: Vegetation is primarily middle-aged forest with patches of early successional grass, } \\
\text { forbs, shrubs, and small trees. }\end{array}$} \\
\hline \multicolumn{3}{|c|}{ Soil: The primary soil type in and around the reference wetland is Sharkey. } \\
\hline \multicolumn{3}{|c|}{$\begin{array}{l}\text { Hydrology: The site receives surface water runoff from the small watershed and backwater } \\
\text { flooding during the winter and spring from a nearby tributary to the Big Sunflower river. }\end{array}$} \\
\hline \multicolumn{3}{|c|}{ Description of Management History / Alterations / Disturbances: } \\
\hline \multicolumn{3}{|l|}{...Pending visit to field site } \\
\hline \multicolumn{3}{|c|}{ Expert Opinion on Condition Relative to Reference Standard: } \\
\hline ...Pending visit to field site & & \\
\hline
\end{tabular}

Figure 19. Example of a Reference Wetland Documentation form. 


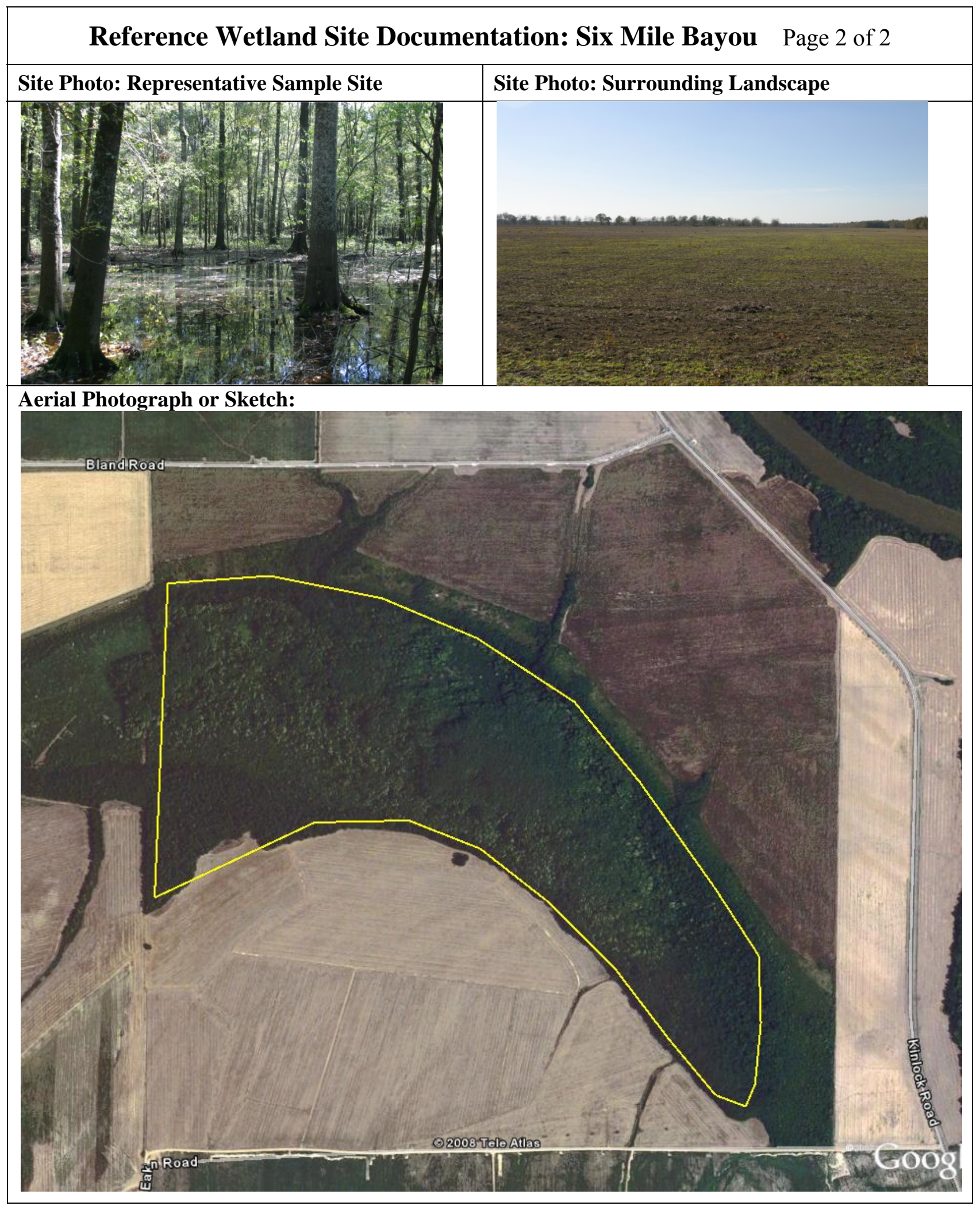

Figure 19. (continued). 


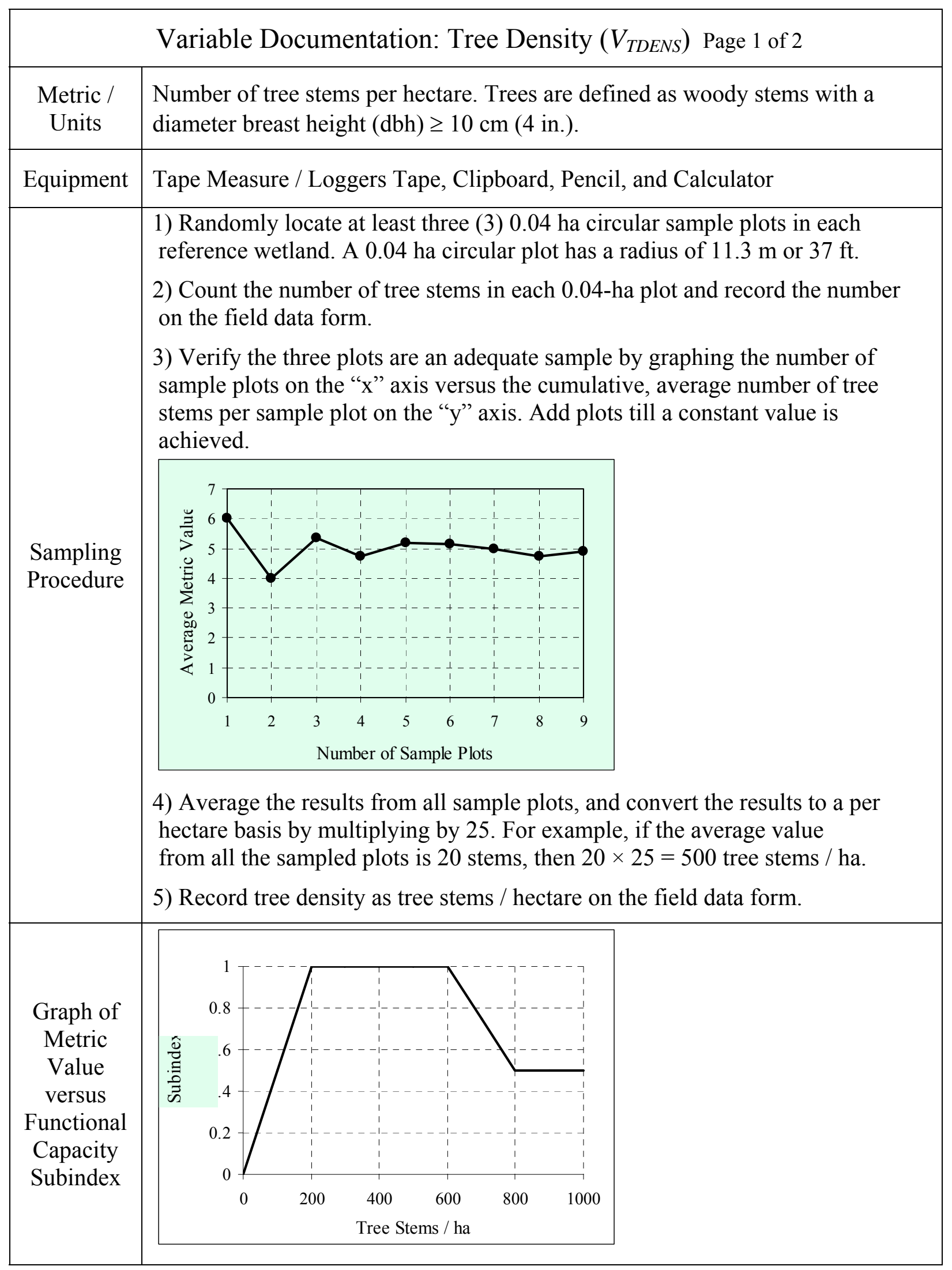

Figure 20. Example of a variable documentation form. 


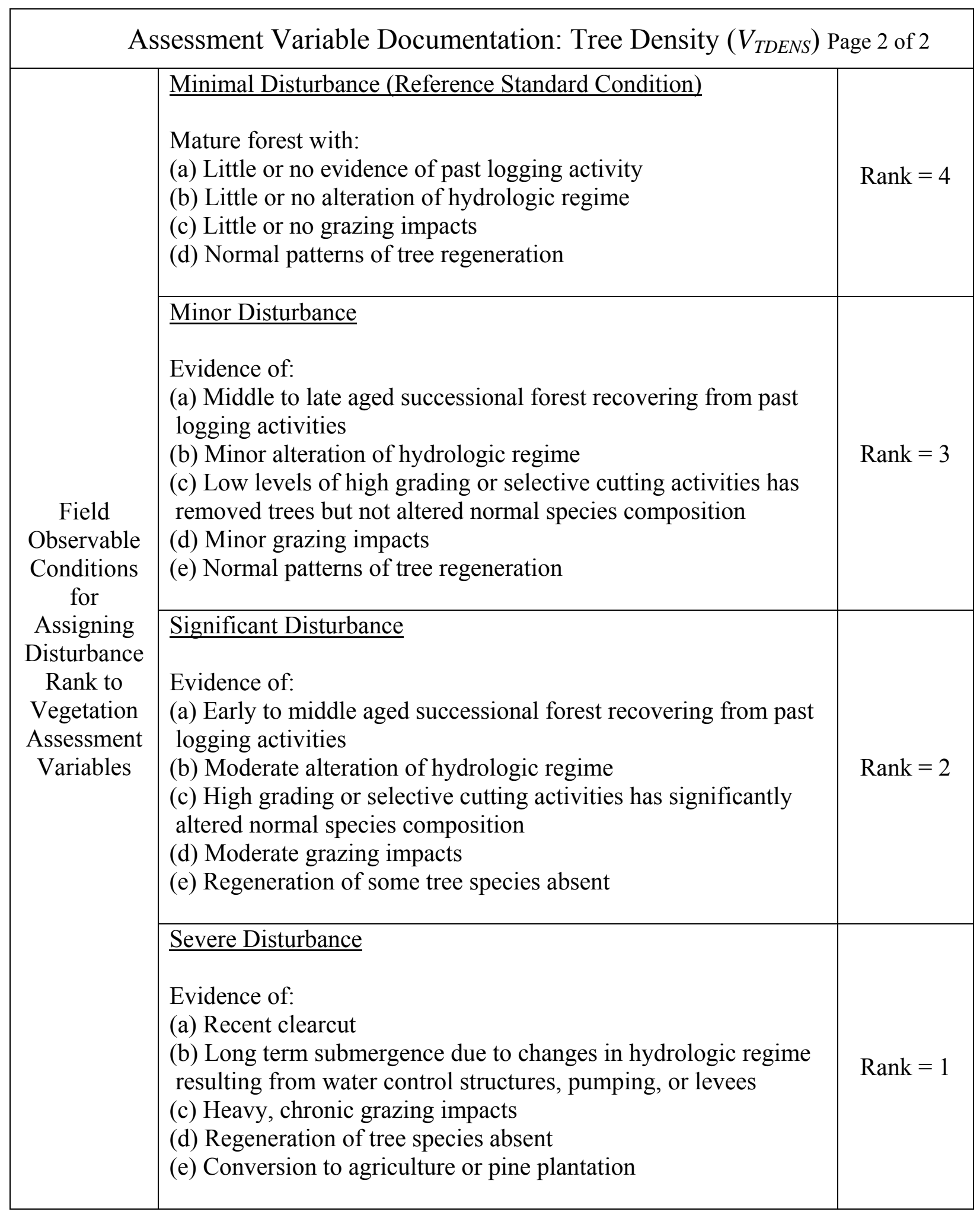

Figure 20. (concluded). 


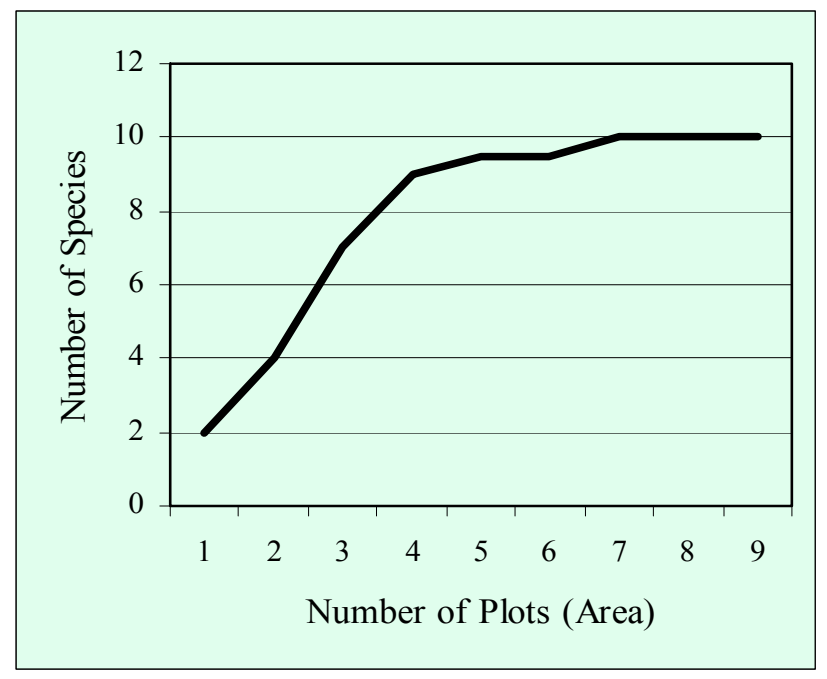

Figure 21. Hypothetical species area curve.

The performance curve is another method for determining the adequacy of sample size by examining the mean metric value from a group of samples. For example, Figure 22 is a hypothetical example of the number of samples plotted on the " $x$ " axis versus the mean metric value plotted on the " $y$ " axis. The graph of metric values typically oscillates at first, and then converges to a relatively constant value at which point the number of samples is adequate to capture the variability exhibited by the metric at the site.

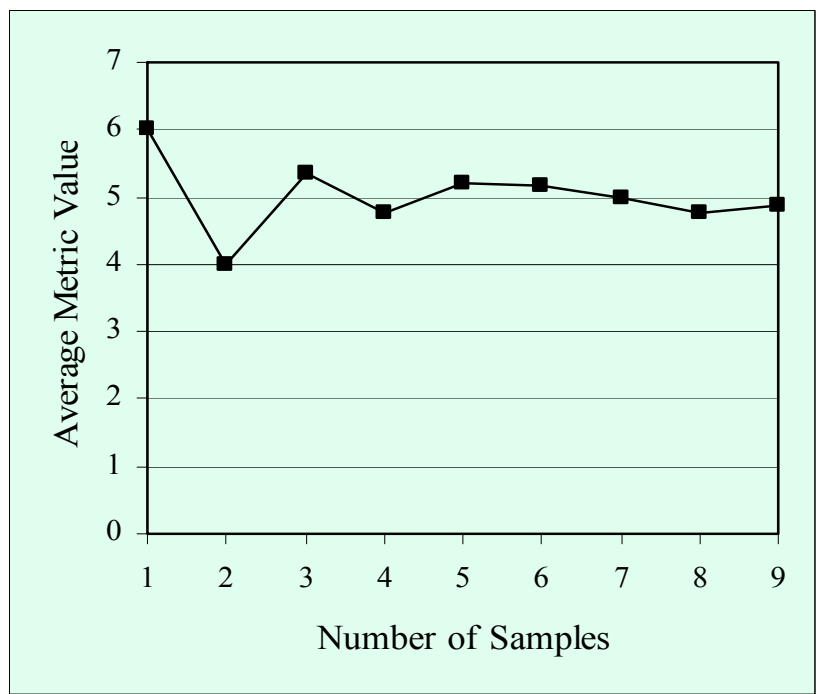

Figure 22. Number of samples versus average metric value.

In the fourth section of the example assessment variable documentation form, a graph of the relationship between the assessment variable metric value and the assessment variable subindex is shown. This represents the 
relationship originally defined by the assessment team during conceptualization of the assessment model (Section titled "Define the Relationship Between Variables and Functional Capacity") and will be subject to revision as reference wetland data are analyzed.

The last section of the example variable documentation form lists the observable field conditions used to assign a disturbance ranking to an assessment variable at each reference wetland. The disturbance ranking is based on the subjective evaluation of field observable conditions that relate to disturbance, structural characteristics, or other factors. Reference wetlands arranged along the human disturbance gradient are used to identify the range of variable metric values that are associated with different portions of the disturbance gradient.

A numeric scale is used to rank the assessment variable along the disturbance gradient, with reference standard conditions assigned the highest numerical rank. The range of the numeric scale should fully capture the range of conditions observed, and the observable field conditions should unambiguously identify the conditions associated with each rank. For example, in the example assessment variable documentation form, a disturbance gradient for vegetation assessment variables is based on conditions related to clear cutting, high grading, or other logging activities, hydrologic regime, grazing, conversions, or other impacts that alter vegetation structure or composition.

Another example of observable field conditions for ranking hydrologic assessment variables along a disturbance gradient is shown in Table 11. Hydrologic assessment variables could also be ranked based on the alteration of parameters such as magnitude, frequency, duration, timing, rate of change, or depth of flow events (Richter et al. 1996, Olden and Poff 2003, Nature Conservancy 2005). Additional examples of ranking wetland sites on a disturbance gradient are provided in Lemly and Rocchio (2009), Rocchio (2007), Apfelbeck and Farris (2005), USEPA (2002, Pages 15-21), and Natural Resources Conservation Service (1999, 2004).

\section{Sampling protocol and field data forms}

The next step in preparing for the collection of wetland reference data is to develop the sampling protocol and field data forms. The sampling protocol is a standardized sequence of steps that ensure that reference wetland data are collected efficiently, accurately, and completely. Field data forms 
Table 11. Conditions for assigning disturbance rank to hydrologic variables (Adapted from Rheinhardt et al. 2007).

\begin{tabular}{|c|c|c|c|}
\hline $\begin{array}{l}\text { Minimally Disturbed - } \\
\text { Reference Standard } \\
\text { Condition }\end{array}$ & Disturbance Minor & Disturbance Significant & Disturbance Severe \\
\hline $\begin{array}{l}\text { Strong evidence of } \\
\text { overbank flow on } \\
\text { floodplain such as: } \\
\text { (a) No apparent } \\
\text { channelization or } \\
\text { incision. } \\
\text { (b) Wrack, sediment, } \\
\text { and/or trash on } \\
\text { floodplain. [Sparse } \\
\text { wrack scores } 45 . \text {.] } \\
\text { (c) High water marks on } \\
\text { trees apparent. } \\
\text { (d) No spoil berm } \\
\text { alongside channel, but } \\
\text { perhaps a natural } \\
\text { levee. }\end{array}$ & $\begin{array}{l}\text { Evidence of occasional } \\
\text { overbank flow on } \\
\text { floodplain such as: } \\
\text { (a) Some wrack, } \\
\text { sediment, trash on } \\
\text { floodplain, but sparse } \\
\text { and/or old. } \\
\text { (b) Stream channelized } \\
\text { within historic channel } \\
\text { with low spoil berms or } \\
\text { breaks in them along } \\
\text { channel. (Channel may } \\
\text { have been channelized } \\
\text { in past, but filled } \\
\text { sufficiently with } \\
\text { sediments that over } \\
\text { bank flow is now } \\
\text { common.) } \\
\text { (c) Channel } \\
\text { channelized or incised } \\
\text { in places. }\end{array}$ & $\begin{array}{l}\text { Evidence of overbank } \\
\text { flow only after extreme } \\
\text { (rare) flood events such } \\
\text { as: } \\
\text { (a) No or little wrack on } \\
\text { floodplain. } \\
\text { (b) Channelization (i.e., } \\
\text { spoil piles/berms } \\
\text { present and high). } \\
\text { (c) Channel incised (not } \\
\text { channelized). } \\
\text { (d) Banks eroding in } \\
\text { places }\end{array}$ & $\begin{array}{l}\text { Overbank flow } \\
\text { eliminated due to: } \\
\text { (a) Deep channelization } \\
\text { with spoil berms } \\
\text { present. } \\
\text { (b) Channel deeply } \\
\text { incised (not } \\
\text { channelized). } \\
\text { (c) Presence of high } \\
\text { artificial levee or other } \\
\text { channel containment } \\
\text { structure. } \\
\text { (d) Extensive bank } \\
\text { erosion and channel } \\
\text { enlargement }\end{array}$ \\
\hline Rank $=4$ & Rank $=3$ & Rank $=2$ & Rank $=1$ \\
\hline
\end{tabular}

provide instructions for collecting individual, or groups of related, assessment-variable metrics, and a place to record data as it is collected. The sampling protocol and field data forms developed for the collection of reference wetland data will later serve as templates for developing the assessment protocol and field data forms that individuals will use to assess wetland functions in conjunction with the guidebook.

There is no standard format for the sampling protocol. However, sampling protocols developed by different groups are often similar. The sampling protocol should be developed by the assessment team after reviewing sampling protocols developed for other guidebooks, and then identifying any unique or special situations associated with the guidebook that may need to be accommodated in the sampling protocol.

Developing the sampling protocol is an iterative process that begins with a list of all information that will be collected at each reference wetland. This 
includes information related to reference wetland documentation as well as variables. Information is arranged into a sequence of steps based on information prerequisites, location requirements (i.e., can the task be accomplished in the office or does it require being in the field), and the spatial scale of the assessment variable (i.e., is the variable sampled based on general observations or based on plot-based sampling). One common sequence is to start with tasks that can be completed in the office, then move to tasks that require general field observation at the reference wetland site, and finally move to tasks that require the collection of data at specific locations in the reference wetland (e.g., plots, subplots, and transects). This approach is illustrated in the example sampling protocol shown in Figure 23.

The sampling protocol begins with preliminary mapping of land use, plant communities, disturbed areas, and other areas of interest, followed by collecting information for assessment variables related to a landscape spatial scale. These tasks are usually easier to accomplish in the office using aerial photographs, topographic maps, and/or when available, a geographic information system (GIS), and help to provide an initial synoptic overview of the reference wetland.

In the second part of the example, the sampling protocol moves to the field for a walking reconnaissance through the reference wetland and the surrounding area. The reconnaissance provides the opportunity to simultaneously verify the preliminary mapping completed in the office, gather information to verify and update the reference wetland documentation form, and collect data for those assessment variables that require qualitative observations at the spatial scale of the reference wetland. In the third part of the example, the sampling protocol moves to the collection of data from plot and transect locations in the reference wetland.

An important part of the field sampling protocol is to provide guidance for selecting the location of sample sites in the reference wetland, and for setting out sample plots and transects at each location. The location of sample sites in a reference wetland can significantly affect whether or not the data collected accurately represent the reference wetland. Consequently, the primary objective in providing guidance for locating sampling sites is to ensure that the locations of sample sites represent the overall conditions in the reference wetland by distributing sample sites throughout the reference wetlands. The resulting sample set will encompass the natural variability 


\section{Sampling Protocol for Collecting Reference Wetland Data Page 1 of 2}

\section{(1) Office Tasks (Use aerial photographs / topographic maps / GIS)}

1.1 - Map and label land uses, plant communities, successional stages, disturbances, hydrologic sources, and unique or distinctive areas in the reference wetland and surrounding area on a photocopy of an aerial photo, topographic map, or other suitable basemap.

1.2 - Determine the metric value for $V_{\text {TRACT }}$ (Field Data Form 1)

1.3 - Determine the metric value for $V_{\text {CORE }}$ (Field Data Form 1)

1.4 - Determine the metric value for $V_{\text {CONNECT }}$ (Field Data Form 1)

1.5 - Assemble the field sampling equipment and supplies (see list on following page)

\section{(2) Conduct Walking Reconnaissance of Reference Wetland and Surroundings}

2.1 - Verify mapping of land uses, plant communities, successional stages, disturbances, hydrologic sources, unique or distinctive areas in the reference wetland and surrounding area

2.2 - Verify and update information on the Reference Wetland Documentation Form

2.3 - Verify metric values for $V_{T R A C T} / V_{C O R E} / V_{C O N N E C T}$ calculated in the office (Field Data Form 1)

2.4 - Determine the metric value for $V_{F R E Q}$ (Field Data Form 1)

2.5 - Determine the metric value for $V_{P O N D}$ (Field Data Form 1)

2.6 - Determine the metric value for $V_{\text {SOIL }}$ (Field Data Form 1)

2.7 - Determine the metric value for $V_{C I C}$ (Field Data Form 1)

\section{(3) Locate and Sample Plot and Transect Assessment Variables}

3.1 - Select sample site locations in reference wetland

3.2 - Layout sample plot, subplots, and transects using the schematic on following page

3.3 - Determine the metric value for $V_{T B A}$ (Field Data Form 2)

3.4 - Determine the metric value for $V_{T D E N}$ (Field Data Form 2)

3.5 - Determine the metric value for $V_{S N A G}$ (Field Data Form 2)

3.6 - Determine the metric value for $V_{\text {TСОМP }}$ (Field Data Form 3)

3.7 - Determine the metric value for $V_{\text {COMP }}$ (Field Data Form 3)

3.8 - Determine the metric value for $V_{W D}$ (Field Data Form 4)

3.9 - Determine the metric value for $V_{L O G}$ (Field Data Form 4)

3.10 - Determine the metric value for $V_{S S D}$ (Field Data Form 5)

3.12 - Determine the metric value for $V_{G V C}$ (Field Data Form 5)

3.13 - Determine the metric value for $V_{O H O R}$ (Field Data Form 6)

3.14 - Determine the metric value for $V_{A H O R}$ (Field Data Form 6)

3.15 - Repeat steps 3.1-3.14 for sample locations \#2 and \#3 and then go to step 3.16

3.16 - Determine adequacy of sample size (see assessment variable documentation -. If sample size is inadequate, repeat Steps 3.1-3.14 at a new sample location and then repeat this step (3.16 - until sample size is adequate.

(4) Check Completeness / Accuracy of Reference Wetland Documentation and Field Data Forms

Figure 23. Example Sampling Protocol (continued). 


\section{Sampling Protocol for Collecting Reference Wetland Data Page 2 of 2}

Plot and Transect Layout Schematic: Randomly select a sample plot location in the reference wetland. Randomly locate subplot centers and transect origins within the plot as shown below

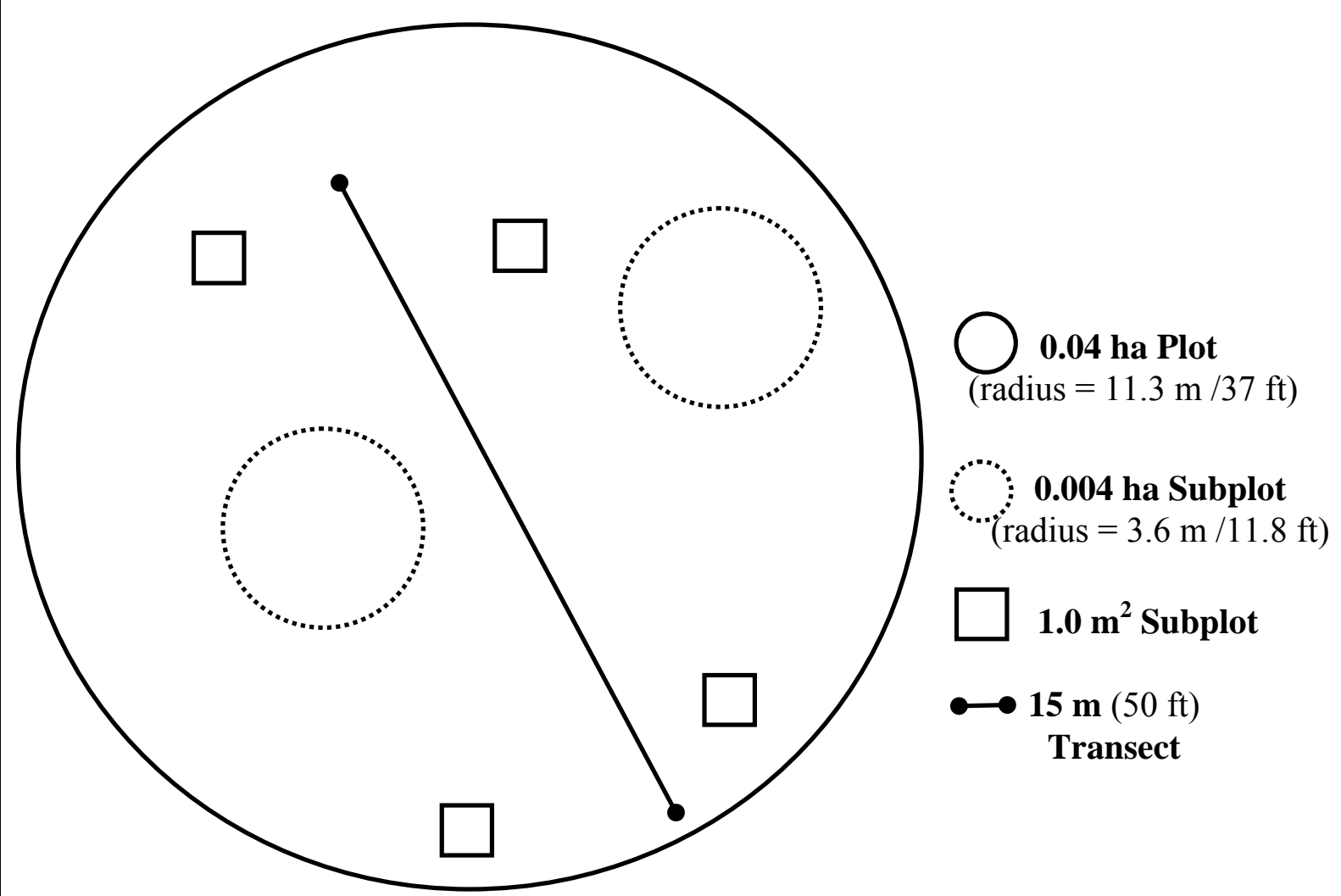

\section{Equipment and Supplies:}

Measuring or Loggers Tape DBH Tape / Calipers

Sampling Frames

Sharpshooter / Soil Probe

Flagging

GPS and Digital Camera / Spare Batteries

Clipboard, Calculator, and Pencils

County Soil Survey

\section{Plant Species}

Acalypha rhomboidea Raf.

Acalypha ostryaefolia Ridd.

Acer negundo L.

Acer saccharinum $\mathrm{L}$.

\begin{tabular}{|c|c|c|}
\hline teries & \multicolumn{2}{|c|}{$\begin{array}{l}\text { Plant Identification Keys } \\
\text { Munsell Soil Color Chart } \\
\text { Aerial Photographs / Topographic Maps } \\
\text { Road Map / Gazetteer } \\
\text { Reference Wetland Site Documentation Form } \\
\text { Field Sampling Protocol } \\
\text { Field Data Forms } \\
\text { Assessment Variable Documentation Forms } \\
\text { Plant Species Code List }\end{array}$} \\
\hline \multicolumn{2}{|l|}{ Common Name } & Code \\
\hline & ACRH \\
\hline \multicolumn{2}{|c|}{ Copper leaf } & ACOS \\
\hline \multicolumn{2}{|l|}{ Box elder } & ACNE \\
\hline \multirow{2}{*}{\multicolumn{2}{|c|}{ Silver maple }} & ACSA \\
\hline & & \\
\hline
\end{tabular}

Figure 23. (concluded). 
within the site, including natural communities as well as small areas of natural disturbance (e.g., wind throw). Possible approaches for identifying sample sites include random, stratified random, or subjective placement. The assessment team should consider the advantages and disadvantages of each of these approaches in terms of the wetland subclass under consideration. A random approach is appropriate if a reference wetland is homogeneous throughout, so that any location is equally likely to be representative of overall conditions. If the reference wetland has areas that are relatively distinctive, such as different plant communities along an environmental gradient, it is better to use a stratified random approach. This involves dividing the reference wetland into the distinctive areas and then randomly identifying sample locations within each of these areas. For example, in some wetland subclasses distinctive plant communities occupy distinct portions of a moisture gradient (i.e., frequently to infrequently flooded). These areas should be defined and sample locations placed randomly in each area. Subjective placement of sample locations should be used with caution because of the tendency to select locations close to a road, and/or away from areas that are difficult to access. Subjective placement is appropriate to relocate random sample locations away from atypical or anomalous situations. Regardless of the method used to identify sample locations, users should verify that an appropriate number of samples are collected using the cumulative count or average value methods described above.

Instructions should be provided for laying out sampling plots, subplots, points, or transects in relation to each sampling site identified. A schematic that illustrates the layout and dimensions of plots, subplots, and transects should be included as part of the sampling protocol (Figure 23). Specific methods should be determined by the assessment team, keeping in mind that methods should be unbiased and easy to apply. One of many possible approaches is to locate subplot centers or transect origins using random distances and directions from the center point of a larger plot or other sample site location.

Two additional items that are useful as part of the field sampling protocol include: a list of the equipment and materials that will be needed in the field for the collection of reference data, and a list of plant species that will likely be encountered (Figure 23). Assign a code to each species that can be used to identify each species on the field data forms. For example, use the first two or three letters of the genus and specific epithet as the species code 
(e.g., Fraxinus pennslvanica $=$ FRPE or FRAPEN). A list of wetland plants with genus, specific epithet, common names, and codes are available for download at the following web sites: http://www.fws.gov/nwi/Plants/plants.htm. http://plants.usda.gov/dl_nrcs_state_plants.html, and https://rsgis.crrel.usace.army.mil/apex/f?p=703.

Once a draft sampling protocol has been completed, field data forms can be developed. Begin by developing a field data form that summarizes the metric values for all assessment variables. Field Data Sheet 1 is an example (Figure 24). Notice that in the example, the sequence in which assessment variables occur on the field form follows the same sequence in which they occur in the sampling protocol (Figure 23). The user simply starts at the beginning of the sampling protocol and is explicitly directed to each succeeding step.

After sorting assessment variables into related groups, users should begin to develop field data forms for individual or related assessment variables. It is likely that multiple field data forms will be required. Design field data forms to be concise and unambiguous. Often this will simply involve transferring information from the assessment variable documentation forms into a format that is suitable for a field data form. The field data form in Figure 24 illustrates some key things to keep in mind when designing field data forms. The first section of each of these field data forms includes information on what data were collected, who collected the data, when the data were collected, and where the data were collected. This essential information should be at the top of all field data forms. The second part of Field Data Form 2 provides instructions for collecting the raw data and other important information such as definitions or plot size. The third part of Field Data Form 2 is where raw data are recorded. The fourth part of Field Data Form 2 provides explicit instructions for converting the raw data into the appropriate metrics and units for further analysis. This part is designated as an office procedure because its completion does not require that one actually be in the field. For quality control purposes, it is a good idea to complete these calculations in the field when they are not overly complex. On return to the office, enter the raw data values into a database or spreadsheet set up to convert raw data into the appropriate metrics and units. Table 12 illustrates a simple spreadsheet for calculating tree basal area from raw field data. 


\section{Assessment Team: \\ Project Name:}

Headwater Slope Wetland HGM Field Data Sheet

Location:

Sampling Date:

Plot Identifier:

Sample variables 1-3 using aerial photography, topographic maps, soil survey maps, etc.

1. $V_{\text {CATCH }}$ Percent change in the size of the catchment (If there is no water diversion or augmentation in the catchment, percent change $=0$ )

Size of original catchment $=$ ha

If diversion: Size of current catchment $=$ ha

If augmentation: Size of catchment from which water is being imported $=\ldots$ ha

2. $V_{\text {UPUSE }}$

Weighted average runoff score for the catchment

...............................

\begin{tabular}{|c|c|c|c|c|}
\hline Land Use & Soil Group & Runoff Score & $\begin{array}{c}\text { Percent (or ha) } \\
\text { in Catchment }\end{array}$ & \\
\hline & & & & \\
\hline & & & & \\
\hline & & & & \\
\hline & & & & \\
\hline & & & & \\
\hline & & & & \\
\hline \multicolumn{4}{|c|}{ Percent of wetland perimeter that is connected to suitable habitat ................... } & $\%$ \\
\hline \multicolumn{4}{|c|}{ Total length of wetland perimeter $=\mathrm{m}$} & \\
\hline \multicolumn{4}{|c|}{ Length of wetland perimeter with suitable habitat $\geq 150 \mathrm{~m}(492 \mathrm{ft})$ wide $=\ldots \mathrm{m}$} & \\
\hline
\end{tabular}

\begin{tabular}{lll}
3 & $V_{\text {CONNECT }}$ & Percent of wetland perimeter that is connected to suitable habitat ............... \\
\cline { 3 - 3 } & Total length of wetland perimeter $=\ldots \mathrm{m}$ \\
\cline { 3 - 3 } & Length of wetland perimeter with suitable habitat $\geq 150 \mathrm{~m}(492 \mathrm{ft})$ wide $=\ldots \mathrm{m}$
\end{tabular}

Sample variable 4 during onsite field reconnaissance

4. $V_{\text {HYDROALT }}$ Height of obstruction, depth of ditch, or depth of impounded water ...

Sample variables 5-11 within one or more representative 0.04-ha (0.1-acre) plot(s) within the WAA

(Use a separate data sheet for each 0.04-ha plot. Report averages across all plots on a separate cover sheet.)

\begin{tabular}{|c|c|c|c|c|c|c|}
\hline \multirow[t]{9}{*}{5 . } & \multirow[t]{9}{*}{$V_{C T D}$} & \multirow{2}{*}{\multicolumn{4}{|c|}{$\begin{array}{l}\text { Average dbh of canopy trees (measure only if total tree cover is } \geq 20 \%) \ldots \ldots \ldots \ldots \\
\text { List dbh measurements of individual canopy trees }(\geq 10 \mathrm{~cm}) \text { below: }\end{array}$}} & $\mathrm{cm}$ \\
\hline & & & & & & \\
\hline & & Subplot 1 & Subplot 2 & Subplot 3 & Subplot 4 & \\
\hline & & & & & & \\
\hline & & & & & & \\
\hline & & & & & & \\
\hline & & & & & & \\
\hline & & & & & & \\
\hline & & & & & & \\
\hline \multirow{3}{*}{6.} & \multirow{3}{*}{$V_{C T D E N}$} & \multirow{2}{*}{\multicolumn{4}{|c|}{ Average number of canopy trees per ha (= canopy trees in 0.04 -ha plot $\times 25) \ldots}}$. & \\
\hline & & & & & & /ha \\
\hline & & \# of canopy trees & ts 1: & & & \\
\hline \multirow[t]{2}{*}{7.} & \multirow[t]{2}{*}{$V_{S S C}$} & \multicolumn{4}{|c|}{ Average percent cover of saplings/shrubs (measure only if tree cover is $<20 \%$ ) ... } & $\%$ \\
\hline & & Subplots 1:_ $\%$ & $\% 4: \ldots \%$ & & & \\
\hline \multirow[t]{2}{*}{8.} & \multirow[t]{2}{*}{$V_{G V C}$} & \multicolumn{4}{|c|}{$\begin{array}{l}\text { Average percent cover of ground-layer vegetation (measure only if tree and } \\
\text { sapling/shrub cover are each }<20 \%) \ldots \ldots \ldots \ldots \ldots \ldots \ldots \ldots \ldots \ldots \ldots \ldots \ldots \ldots \ldots \ldots\end{array}$} & $\%$ \\
\hline & & \multicolumn{4}{|c|}{ Subplots 1: _ $\% 2: \_\% 3: \_\% 4: \_\%$} & \\
\hline
\end{tabular}

Remarks:

Figure 24. Example Field Data Form for summarizing assessment variable metric values (continued). 


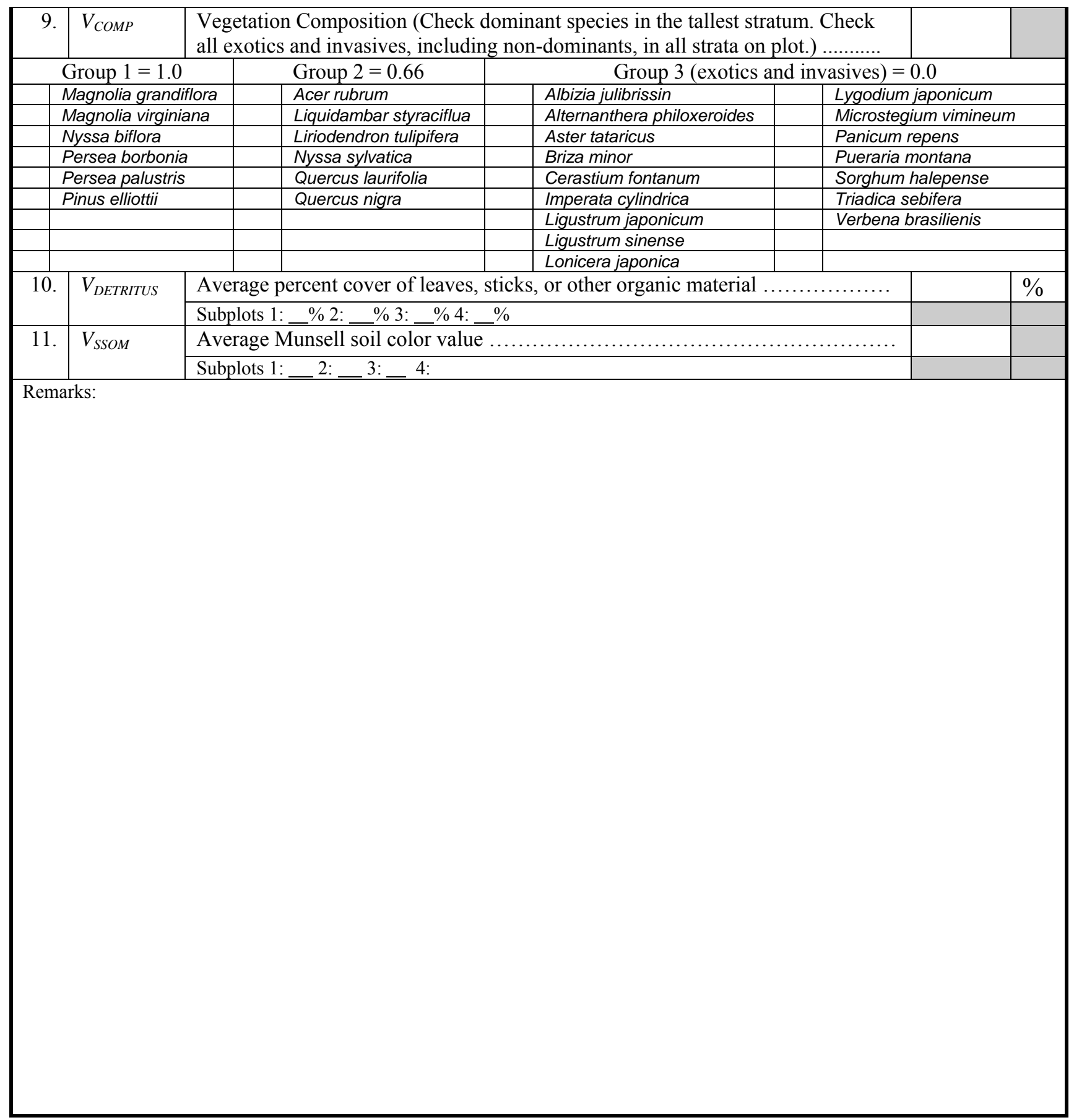

Figure 24. (concluded). 
Table 12. Example of a spreadsheet for calculating tree basal area.

\begin{tabular}{|c|c|c|c|c|c|}
\hline & $A$ & B & C & $D$ & $E$ \\
\hline 1 & $\begin{array}{l}\text { Enter Individual } \\
\text { Tree Species Code } \\
\text { in Cells A2-A15 }\end{array}$ & $\begin{array}{l}\text { Enter Individual } \\
\text { Tree Diameters } \\
\text { (cm) in Cells B2- } \\
\text { B15 }\end{array}$ & $\begin{array}{l}\text { Convert to } \mathrm{cm}^{2} / \\
0.04 \text { ha (Formula: } \\
0.25 * 3.14 * \text { tree } \\
\text { diameter }{ }^{2}=\mathrm{cm}^{2} \text { ) }\end{array}$ & $\begin{array}{l}\text { Convert to } \mathrm{m}^{2} / \\
0.04 \text { ha (Formula: } \\
\text { Column } \mathrm{B} * \\
\left.0.0001=\mathrm{m}^{2}\right)\end{array}$ & $\begin{array}{l}\text { Convert to } \mathrm{m}^{2} / \text { ha } \\
\text { (Formula: Column } \\
\mathrm{C} * 25=\mathrm{m}^{2} / \mathrm{ha}\end{array}$ \\
\hline 2 & FRPE & 12 & 113.04 & 0.01 & 0.28 \\
\hline 3 & FRPE & 8 & 50.24 & 0.01 & 0.13 \\
\hline 4 & QULY & 13 & 132.67 & 0.01 & 0.33 \\
\hline 5 & QULY & 23 & 415.27 & 0.04 & 1.04 \\
\hline 6 & QULY & 25 & 490.63 & 0.05 & 1.23 \\
\hline 7 & CAAQ & 21 & 346.19 & 0.03 & 0.87 \\
\hline 8 & CAAQ & 14 & 153.86 & 0.02 & 0.38 \\
\hline 9 & QUNU & 23 & 415.27 & 0.04 & 1.04 \\
\hline 10 & QUNU & 45 & 1589.63 & 0.16 & 3.97 \\
\hline 11 & QUNU & 34 & 907.46 & 0.09 & 2.27 \\
\hline 12 & QUNU & 17 & 226.87 & 0.02 & 0.57 \\
\hline 13 & & & 0 & 0 & 0 \\
\hline 14 & & & 0 & 0 & 0 \\
\hline 15 & & & 0 & 0 & 0 \\
\hline 16 & \multicolumn{4}{|c|}{ Total Tree Basal Area in $\mathrm{m}^{2} / \mathrm{ha}=$} & 16.86 \\
\hline
\end{tabular}

For some assessment variables, the calculations and conversions can be quite complex; consequently, calculation in the field is time-consuming and error-prone (Table 13). For these assessment variables, the raw data should be taken to the office and entered into a spreadsheet set up to do the required calculations.

Compare the values from the field data form and spreadsheet for accuracy and make corrections, or update, the field forms if necessary. In addition to ensuring accuracy, this quality control procedure has the additional benefit that the reference wetland data are now archived in both hardcopy and digital formats.

The last part of Data Form 2 is used to rank the reference wetland relative to reference standard conditions, and describe the condition on which the rank was assigned. The conditions associated with specific ranks are described on the assessment variable documentation form (Figure 20). 
Table 13. Example of a spreadsheet for woody debris and log volume metric value calculations and conversions.

\begin{tabular}{|c|c|c|c|c|c|c|c|c|c|c|c|c|c|c|c|c|c|c|c|c|}
\hline A & \multicolumn{2}{|c|}{ B } & C & D & \multicolumn{2}{|c|}{$E$} & $\mathrm{~F}$ & G & \multicolumn{2}{|c|}{$\mathrm{H}$} & I & $J$ & K & L & M & $\mathrm{N}$ & 0 & $P$ & & Q \\
\hline & \multicolumn{2}{|c|}{$\begin{array}{c}\text { Size Class } 1 \text { No. } \\
\text { of Stems / } \\
\text { Transect }\end{array}$} & \multirow{2}{*}{\begin{tabular}{|c|} 
Size \\
Class 1 \\
Average \\
No. \\
Stems / \\
Trnsect
\end{tabular}} & \multirow{2}{*}{$\begin{array}{c}\text { Size } \\
\text { Class } 1 \\
\text { tons/ } \\
\text { acre }\end{array}$} & \multicolumn{2}{|c|}{$\begin{array}{c}\text { Size Class } 2 \\
\text { No. of Stems / } \\
\text { Transect }\end{array}$} & \multirow{2}{*}{$\begin{array}{c}\text { Size } \\
\text { Class } 2 \\
\text { Average } \\
\text { No. } \\
\text { Stems / } \\
\text { Trnsect }\end{array}$} & \multirow{2}{*}{$\begin{array}{c}\text { Size } \\
\text { Class } 2 \\
\text { tons/ } \\
\text { acre }\end{array}$} & \multicolumn{2}{|c|}{$\begin{array}{c}\text { Size Class } 3 \text { Sum } \\
\text { of Stem Area } \\
\left(\mathrm{cm}^{2}\right)\end{array}$} & \multirow{2}{*}{$\begin{array}{c}\text { Size } \\
\text { Class } 3 \\
\text { Average } \\
\text { of Stem } \\
\text { Area } \\
\left(\mathrm{cm}^{2}\right)\end{array}$} & \multirow{2}{*}{\begin{tabular}{|c|} 
Size \\
Class 3 \\
tons/ \\
acre
\end{tabular}} & \multirow[b]{2}{*}{$\mid \begin{array}{l}\text { Size Class } \\
3 \mathrm{ft}^{3} / \mathrm{acre}\end{array}$} & & \multirow{2}{*}{$\begin{array}{l}\left(V_{\text {LOG }}\right) \\
\text { Size } \\
\text { Class } \\
3 \mathrm{~m}^{3} / \mathrm{ha}\end{array}$} & \multirow[b]{2}{*}{$\mid \begin{array}{c}\text { Size Class } \\
1,2 \text {, and } \\
3 \mathrm{ft}^{3} / \text { acre }\end{array}$} & \multirow[b]{2}{*}{$\mid \begin{array}{c}\text { Size Class } \\
1,2 \text {, and } \\
3 \mathrm{ft}^{3} / \text { acre }\end{array}$} & & \multirow{2}{*}{\multicolumn{2}{|c|}{$\begin{array}{c}\left(V_{w D}\right) \text { Size Class } \\
1,2 \text {, and } \\
3 \mathrm{~m}^{3} / \mathrm{ha}\end{array}$}} \\
\hline & $\begin{array}{c}\text { Trnsect } \\
1\end{array}$ & $\left|\begin{array}{c}\text { Trnsect } \\
2\end{array}\right|$ & & & $\mid \begin{array}{c}\text { Trnsect } \\
1\end{array}$ & $\begin{array}{c}\text { Trnsect } \\
2\end{array}$ & & & $\begin{array}{c}\text { Trnsect } \\
1\end{array}$ & $\begin{array}{c}\text { Trnsect } \\
2\end{array}$ & & & & & & & & & & \\
\hline Plot 1 & 5 & 9 & 7 & 0.6545 & 2 & 6 & 4 & 1.784 & 3573.32 & 9012.59 & 6292.95 & 432.33 & 23889.7 & Plot 1 & 1648.39 & 434.76 & 24024.48 & Plot 1 & 1657.69 & \\
\hline Plot 2 & & & 0 & 0 & & & 0 & 0 & 0.00 & 0.00 & 0.00 & 0.00 & 0.00 & Plot 2 & 0.00 & 0.00 & 0.00 & Plot 2 & 0.00 & \\
\hline Plot 3 & & & 0 & 0 & & & 0 & 0 & 3.14 & 0.00 & 1.57 & 0.11 & 5.96 & Plot 3 & 0.41 & 0.11 & 5.96 & Plot 3 & 0.41 & \\
\hline Plot 4 & & & 0 & 0 & & & 0 & 0 & 0.00 & 0.00 & 0.00 & 0.00 & 0.00 & Plot 4 & 0.00 & 0.00 & 0.00 & Plot 4 & 0.00 & \\
\hline \multirow[t]{3}{*}{ Plot 5} & & & 0 & 0 & & & 0 & 0 & 0.00 & 0.00 & 0.00 & 0.00 & 0.00 & Plot 5 & 0.00 & 0.00 & 0.00 & Plot 5 & 0.00 & \\
\hline & \multicolumn{2}{|c|}{$\begin{array}{c}\text { Size Class } 3 \\
\text { Stem Diameters } \\
\text { Plot } 1\end{array}$} & \multicolumn{2}{|c|}{$\begin{array}{l}\text { Size Class } 3 \text { Stem } \\
\text { Area }(\mathrm{cm} 2) \text { Plot } 1\end{array}$} & \multicolumn{2}{|c|}{$\begin{array}{c}\text { Size Class } 3 \\
\text { Stem Diameters } \\
\text { Plot } 2\end{array}$} & \multicolumn{2}{|c|}{$\begin{array}{c}\text { Size Class } 3 \\
\text { Stem Area }(\mathrm{cm} 2) \\
\text { Plot } 2\end{array}$} & \multicolumn{2}{|c|}{$\begin{array}{l}\text { Size Class } 3 \text { Stem } \\
\text { Diameters Plot } 3\end{array}$} & \multicolumn{2}{|c|}{$\begin{array}{c}\text { Size Class } 3 \\
\text { Stem Area }(\mathrm{cm} 2) \\
\text { Plot } 3\end{array}$} & \multicolumn{2}{|c|}{$\begin{array}{c}\text { Size Class } 3 \text { Stem } \\
\text { Diameters Plot } 4\end{array}$} & \multicolumn{2}{|c|}{$\begin{array}{l}\text { Size Class } 3 \text { Stem } \\
\text { Area }(\mathrm{cm} 2) \text { Plot } 4\end{array}$} & \multicolumn{2}{|c|}{$\begin{array}{l}\text { Size Class } 3 \text { Stem } \\
\text { Diameters Plot } 5\end{array}$} & \multicolumn{2}{|c|}{$\begin{array}{c}\text { Size Class } 3 \\
\text { Stem Area }(\mathrm{cm} 2) \\
\underline{\text { Plot } 5}\end{array}$} \\
\hline & $\begin{array}{c}\text { Trnsect } \\
1\end{array}$ & $\left|\begin{array}{c}\text { Trnsect } \\
2\end{array}\right|$ & $\begin{array}{c}\text { Trnsect } \\
1\end{array}$ & $\begin{array}{c}\text { Trnsect } \\
2\end{array}$ & $\begin{array}{c}\text { Trnsect } \\
1\end{array}$ & $\begin{array}{c}\text { Trnsect } \\
2\end{array}$ & $\begin{array}{c}\text { Trnsect } \\
1\end{array}$ & $\begin{array}{c}\text { Trnsect } \\
2\end{array}$ & $\begin{array}{c}\text { Trnsect } \\
1\end{array}$ & $\begin{array}{c}\text { Trnsect } \\
2\end{array}$ & $\begin{array}{c}\text { Trnsect } \\
1\end{array}$ & $\left|\begin{array}{c}\text { Trnsect } \\
2\end{array}\right|$ & $\begin{array}{c}\text { Trnsect } \\
1\end{array}$ & $\left|\begin{array}{c}\text { Trnsect } \\
2\end{array}\right|$ & $\begin{array}{c}\text { Trnsect } \\
1\end{array}$ & $\begin{array}{c}\text { Trnsect } \\
2\end{array}$ & $\begin{array}{c}\text { Trnsect } \\
1\end{array}$ & $\begin{array}{c}\text { Trnsect } \\
2\end{array}$ & $\begin{array}{c}\text { Trnsect } \\
1\end{array}$ & $\begin{array}{c}\text { Trnsect } \\
2\end{array}$ \\
\hline Plot 1 & 4.00 & 34.00 & 12.56 & 907.46 & & & 0.00 & 0.00 & & & 0.00 & 0.00 & & & 0.00 & 0.00 & & & 0.00 & 0.00 \\
\hline Plot 2 & 10.00 & 4.00 & 78.50 & 12.56 & & & 0.00 & 0.00 & & & 3.14 & 0.00 & & & 0.00 & 0.00 & & & 0.00 & 0.00 \\
\hline Plot 3 & 20.00 & 56.00 & 314.00 & 2461.76 & & & 0.00 & 0.00 & & & 0.00 & 0.00 & & & 0.00 & 0.00 & & & 0.00 & 0.00 \\
\hline Plot 4 & 30.00 & 78.00 & 706.50 & 4775.94 & & & 0.00 & 0.00 & & & 0.00 & 0.00 & & & 0.00 & 0.00 & & & 0.00 & 0.00 \\
\hline Plot 5 & 56.00 & 33.00 & 2461.76 & 854.87 & & & 0.00 & 0.00 & & & 0.00 & 0.00 & & & 0.00 & 0.00 & & & 0.00 & 0.00 \\
\hline & & & 3573.32 & 9012.59 & & & 0.00 & 0.00 & & & 3.14 & 0.00 & & & 0.00 & 0.00 & & & 0.00 & 0.00 \\
\hline
\end{tabular}


Field Data Forms 1 and 2 also illustrate several features that can make data forms simple and efficient. First, start the data collection process at the top of the page and continue sequentially down the page whenever possible. Moving around to different parts of a page, or between pages, is timeconsuming and can lead to errors. Second, break down complex procedures into multiple, simple steps. Third, always include units. Fourth, include a place for recording results of intermediate steps. When errors are made, this helps to track down where the error occurred. Fifth, using shading (Figure 24) on the data forms can make it easier to identify specific parts of the data form and keep attention focused on the appropriate place. Finally, Data Form 1 is an example of a data form that gathers information from many different data forms and summarizes them into a single data form. Gathering all the metric values onto a summary data form makes it easy to transcribe the information to a spreadsheet, or other software, for analysis and archiving. For example, on Data Form 2, Step 11 of the Office Procedure directs the data collector to transfer the final metric values for tree basal area $\left(\mathrm{V}_{\mathrm{TBA}}\right)$ to Data Form 1.

The field sampling protocol and field data forms should be field-tested as a quality control opportunity to identify and correct problems, and provide the opportunity to "fine tune" the protocol. Thorough field-testing will help to avoid the all-too-common situation where after sampling 20 reference wetlands, a problem is identified that necessitates revisiting and resampling. The following steps should be included as part of testing the field sampling protocol. First, verify that the sequence of tasks identified in the field sampling protocol is logical and efficient. If necessary, re-order the sequence of tasks to optimize movement around the site and the time it takes to transition from sampling one variable to another. Second, work through the sampling procedure for each metric on the field data forms. Check to make sure the steps in each sampling procedure follow a logical sequence, verify that the steps of the sampling procedure are consistent with steps identified on the assessment variable documentation forms (Chapter 4), that the instructions at each step explicitly indicate what to do next, that units are included and are correct, conversion formulas are correct, and that where to record data and/or summarize results is clearly identified. Rearrange, add, or delete steps on the field forms if necessary to simplify, clarify, or increase efficiency. Third, verify that the equipment, material, and field data form lists of the sampling protocol include all necessary items. Fourth, update the plant species codes list on the sampling protocol form. Fifth, verify that instructions for identifying plot and transect locations are 
explicit and that plot and transect schematics are correct, including the dimensions. Sixth, for quality control purposes, return to the office and enter raw data from field data forms into spreadsheets set up to reproduce calculations and conversions on field data forms.

\section{Collect reference wetland data}

After the field sampling protocol and field data forms have been developed and field tested, the collection of reference wetlands data can begin. Consider conducting a pilot study of a small number of reference wetlands that represent the full range of human disturbance exhibited in the wetland subclass. A pilot study is a good way to field-test the efficacy of the wetland reference documentation forms, the sampling protocol, and the field data forms. This will help to proactively identify potential problems, needs, constraints, or reveal any insights that are likely to arise during a full-scale implementation. Once any confusion, problems, or errors that are identified have been resolved, reference wetland data can be collected from the remaining reference wetlands.

\section{Manage reference wetland data}

Management of reference data entails supervising the flow of information from the field data forms, to digital formats, and through the various types of data analysis. It includes the archiving of field data forms, transcribing reference wetland data from the field data forms to a digital format, using databases and spreadsheets to convert raw metric data to the appropriate units, and using spreadsheets, or other software, to analyze reference wetland data. Each of these aspects of reference data management is discussed below. Examples will be provided, but it is beyond the scope of this chapter to provide tutorials for spreadsheet, database, and other software that may be used to manage reference data.

The collection of field data requires significant time and effort. Make duplicate copies of the original field data forms, and file them in several safe places with other materials related to the development of the guidebook (e.g., reference wetland and assessment variable documentation). Another option is to scan the original field data forms and keep copies of the files on different hard drives. Make the duplicates as the field data forms are finalized. Do not wait until all reference data have been collected. 
Put some time and thought into the way the raw field data are entered to ensure that they can be easily linked, copied, or otherwise transferred for further analysis. Begin by identifying the different types of analysis that need to be accomplished, and then design databases or spreadsheets to accomplish each task. During the design process, consider how information will be transferred (e.g., cell links, cut and paste, etc.) between spreadsheets or exported to other software. The objective is to enter the raw data just once, and then manipulate the data digitally for use in further analysis.

Three basic types of spreadsheets are typically useful in managing reference wetland data. The first type of spreadsheet is used to convert and summarize raw metric data to the appropriate units. Table 12 is an example of a relatively simple spreadsheet that converts and summarizes individual tree diameter measurements into units of $\mathrm{m}^{2} / \mathrm{ha}$. Table 13 is a more complex spreadsheet that converts and summarizes number of stems (size classes 1 and 2) and stem diameter (size class 3 ) measurements into units of $\mathrm{m}^{3} / \mathrm{ha}$.

The second type of spreadsheet summarizes the final metric values for all assessment variables. This type of spreadsheet is used in scaling metrics from the fully functional reference condition found in undisturbed reference wetlands to the less functional conditions found in reference wetlands with various levels of disturbance. This spreadsheet can be linked to the spreadsheets that are used to convert and summarize raw field data (Figure 24) in order to avoid re-entering information.

The third type of spreadsheet is used to calculate subindex values and Functional Capacity Indices (FCI). In this spreadsheet, the final metric values from a reference wetland are entered in the lightly shaded cells and the subindex (moderately shaded cells) and FCI values (darkly shaded cells) are displayed. This spreadsheet can be linked to the summary spreadsheet or information can be cut and pasted to avoid re-entering information.

Quality control is an integral part of the collection and management of reference wetland data. Quality control consists of procedures designed to ensure consistency, accuracy, and completeness in the collection and management of reference wetland data. It is the responsibility of the assessment team to identify and implement a suite of quality control procedures that address the following quality control issues. 
Training: Individuals collecting reference wetland data should be trained in all aspects of reference wetland data collection. This includes wetland reference documentation forms, assessment variable documentation forms, the sampling protocol, selection of the sample site location, the layout of plot and transects, use of field equipment, and sampling procedures. Novices should be paired with experienced individuals until a complete understanding of all aspects of reference wetland data collection, under a variety of field situations, has been achieved.

Field data completeness and accuracy: The last step of the sampling protocol is to conduct a completeness and accuracy check of the field data forms and the reference wetland documentation form. This quality control step ensures that the field data forms and reference wetland documentation form are complete, accurate, and legible prior to leaving a reference wetland.

Data maintenance and archiving: Maintaining, updating, and archiving reference wetland data should be overseen by a single individual. Field data forms should be passed to this person, whose responsibilities include duplicating, transcribing to digital formats, updating, and archiving of reference wetland documentation forms, assessment variable documentation forms, field data forms, spreadsheets, and other types of files used during the collection and management of reference wetland data.

Data Transcription: Data should be transcribed from field data forms to a digital format by two people. One person should read from the data forms while the other person enters the data into a digital format. To check for transcription errors, the process should be repeated with one person reading from the data forms and the second person checking the digital data previously entered. 


\section{Calibrate, Verify, and Field-Test Assessment Models}

\section{Introduction}

As discussed previously, assessment models are developed in three distinct phases in the HGM Approach. The first phase involves conceptualizing assessment models based on the personal knowledge and experience of the assessment team, along with existing literature and data. The second phase, discussed in this chapter, involves calibrating, verifying, and fieldtesting the assessment models. This includes a variety of tasks such as screening variables for their discriminatory power, calibrating metrics to the range of conditions exhibited by reference wetlands, verifying the logic and sensitivity of the assessment models, and field-testing assessment models. These tasks are relatively easy to accomplish once reference wetland data have been collected. For example, a test of model logic and sensitivity can be accomplished in less than an hour, and the results can be used immediately to guide the revision of conceptual assessment models.

During the third phase, calibrated assessment models are validated using independent, quantitative measures of function. For example, an independent measure of function for the capacity of a wetland to retain sediments could be the amount of sediment accretion in a specified area over a specified period of time (Kleiss 1996). The validation of assessment models is generally considered to be beyond the scope and responsibility of the assessment team because validation is often a complex and time-consuming process that can involve years of additional research and data collection (Pohll et al. 1999, Carroll et al. 2000, Hill et al. 2006, Bauder et al. 2009). Validation of assessment models is discussed in Chapter 7, "Validate Assessment Models.”

\section{Test suitability of variables and metrics}

The assessment team initially selects variables during conceptualization of the assessment models. At the same time, the assessment team defines the relationship between metric values and functional capacity (Chapter 3, "Conceptualize Assessment Models"). Following the collection of reference wetland data, metrics are screened to determine their ability to discriminate between various types and degrees of disturbance that occur across the 
range of conditions exhibited in reference wetlands. For those metrics that provide sufficient discrimination, the relationship between each metric and functional capacity is calibrated based on the analysis of reference wetland data.

A variety of criteria can be used to test metric suitability. Karr et al. (1997), Hughes et al. (1998), McCormick et al. (2001), USEPA (2002), Klemm et al. (2003) and Whittier et al. (2007) discuss various criteria in the context of multimetric indices such as Indicators of Biological Integrity (IBI). These include the range of metric values, variability (i.e., signal-to-noise ratio), correlation to natural gradients, responsiveness, and redundancy. Not all of these screening criteria are necessarily appropriate for screening metrics in the HGM Approach. The assessment team should consider multiple criteria and determine whether or not they are an appropriate method for screening a specific metric.

\section{Range of metric values}

The initial step in determining metric suitability is to visually examine a scatter graph of metric values to get a sense of patterns and trends in the data. This is accomplished by plotting metric values (y-axis) against reference wetland sites sorted by ascending order of the metric value (x-axis). Figure 25 is an example in which the metric values exhibit a relatively narrow range of values, and Figures 26 and 27 are examples in which the metric values exhibit a wider range of values. Generally, metrics that exhibit a narrow range of values do not discriminate between wetlands with high versus low functional capacity and should be discarded. Metrics with a wide range are often better able to discriminate between the two, and should be subjected to further screening. Model developers must decide what constitutes a suitable range of values for each metric. For example, in evaluating candidate metrics, Whittier et al. (2007) eliminated richness metrics with a range of less than four species, and any metric where more than $75 \%$ of the metric values were identical.

The next step is to plot a scatter graph of metric values (y-axis) against the disturbance gradient categories (x-axis) assigned to reference wetlands during the collection of reference wetland data as discussed in Chapter 4, "Reference Wetlands and Reference Wetland Data." Remember that the disturbance gradient represents an a priori FCI, based on the knowledge and experience of the assessment team that provides an initial basis for ordering metric values. Figure 28 is a scatter graph of the metric values 


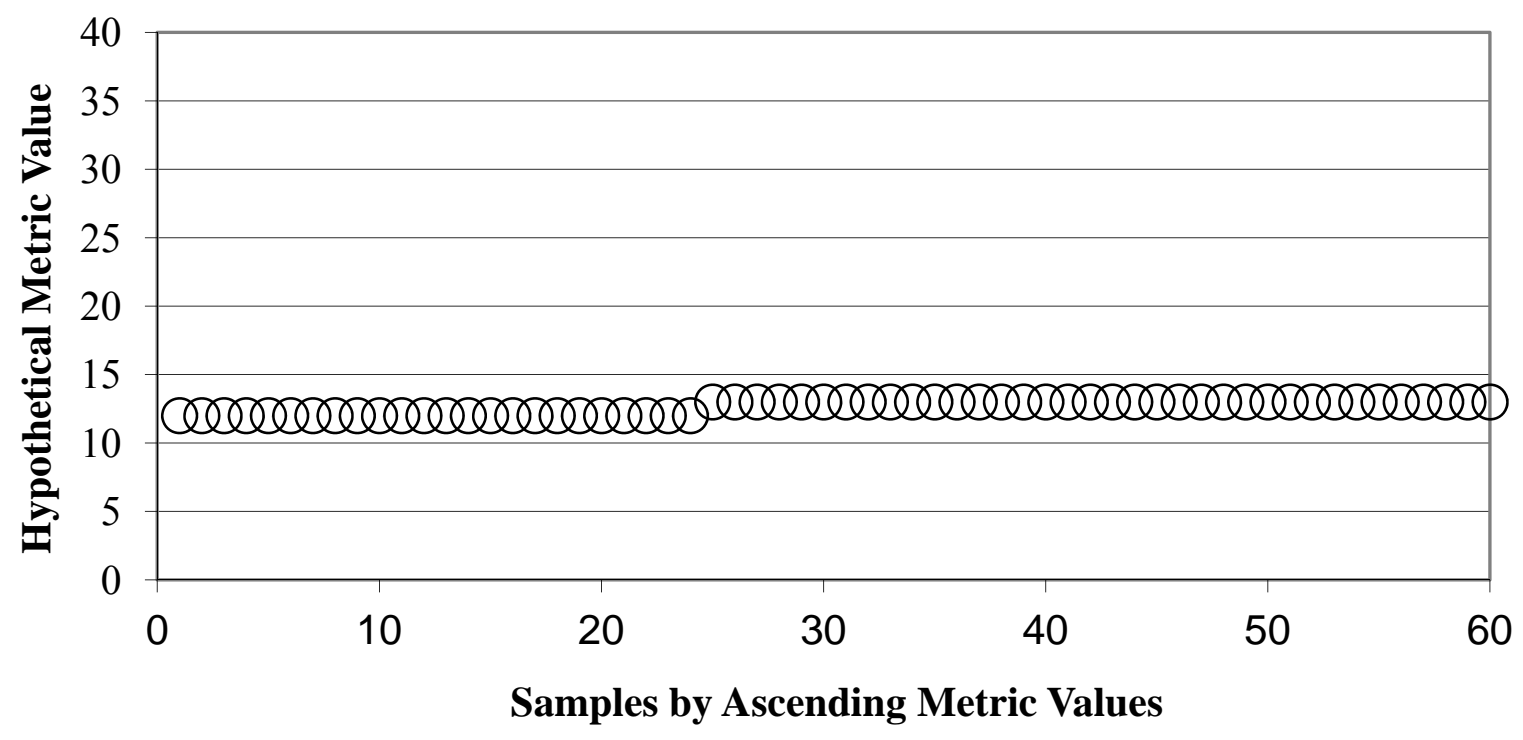

Figure 25. Scatter graph of a narrow range of hypothetical metric values and samples sorted by ascending metric values.

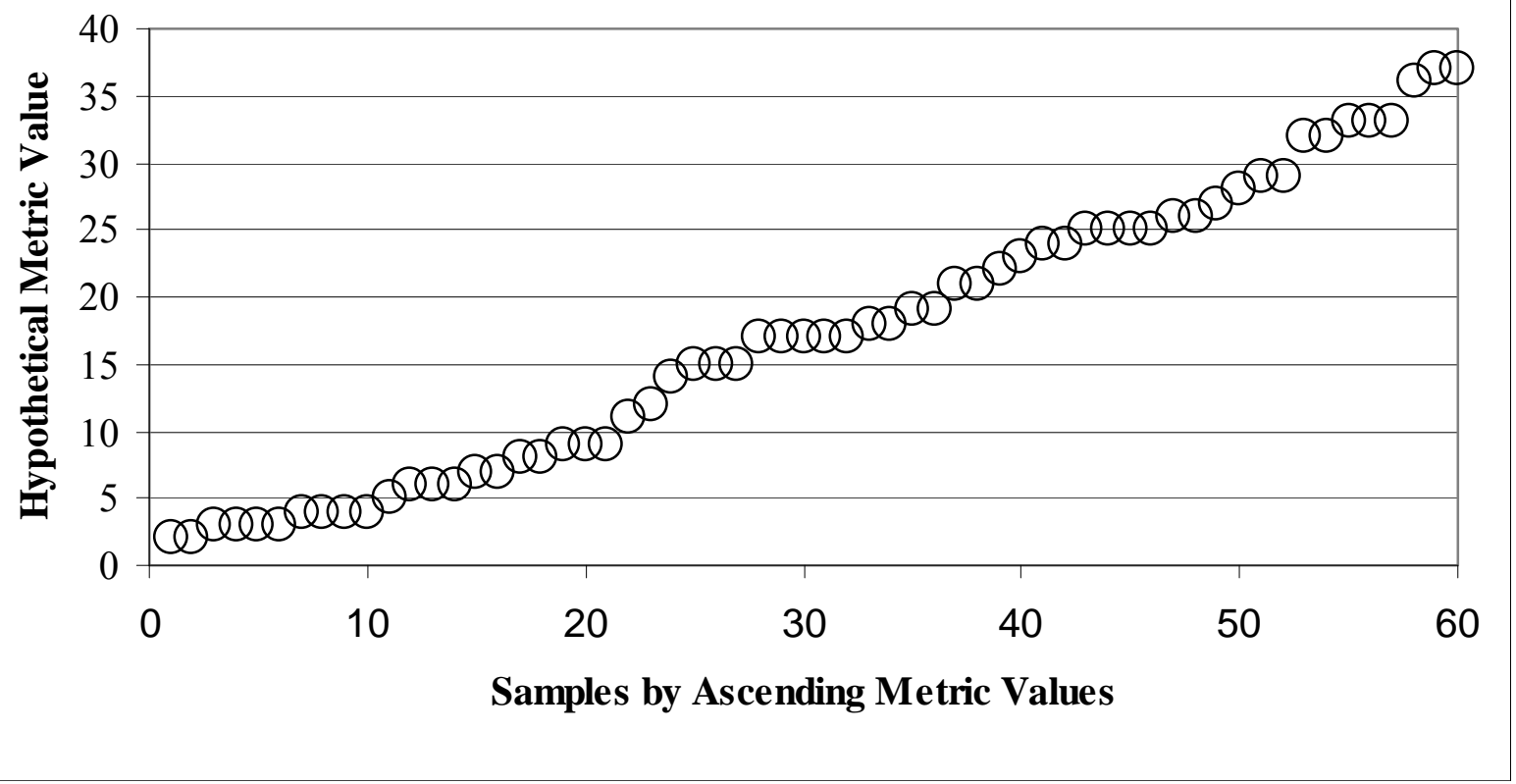

Figure 26. Scatter graph of a wider range of hypothetical metric values and samples sorted by ascending metric values. 


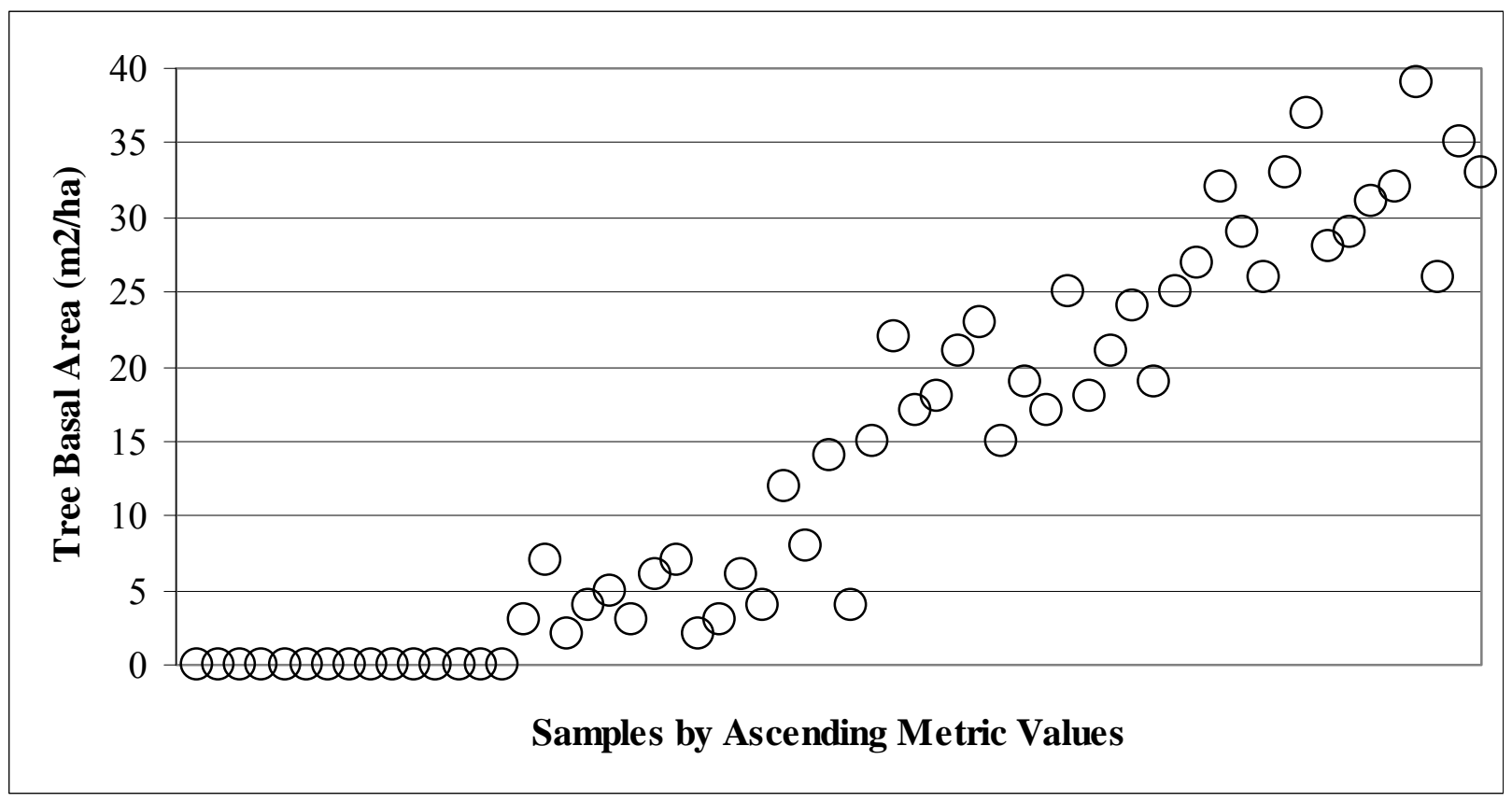

Figure 27. Scatter graph of a tree basal area metric values and sample number (sorted ascending).

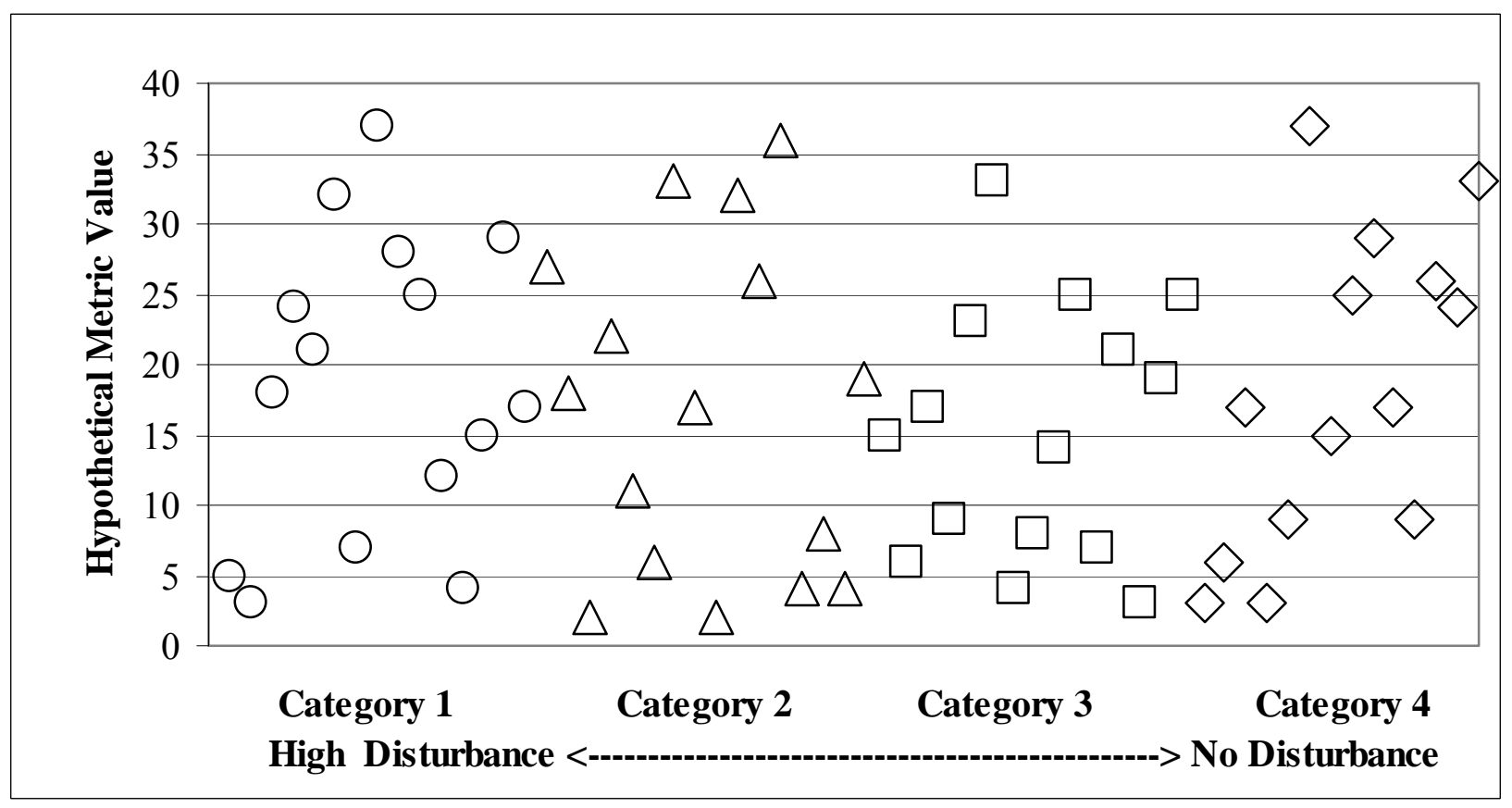

Figure 28. Hypothetical metric value in relation to disturbance categories.

from Figure 26 plotted against the disturbance gradient categories. There appears to be a random pattern with no clear relationship between metric values and the disturbance gradient because all categories exhibit a similar wide range of metric values. This suggests that this particular metric fails to discriminate between sites exhibiting high and low functionality. Figure 29 
is a scatter graph of tree basal area metric values from Figure 27 plotted against the disturbance gradient categories. In this case, the metric values exhibit a linear trend with basal area decreasing with increasing disturbance. Moving from left to right in the graph, an interpretation of the pattern can be formulated. Reference wetlands in the first quarter of the graph are sites that were recently logged or converted to agriculture. In these areas, no trees are present and basal area is $0.0 \mathrm{~m}^{2} / \mathrm{ha}$. Reference wetlands in the second quarter are sites undergoing early succession with basal areas $<15 \mathrm{~m}^{2} /$ ha. Reference wetlands in the third quarter are sites that have been high graded with basal areas between 15 and $25 \mathrm{~m}^{2} / \mathrm{ha}$, and reference wetlands in the fourth quarter of the graph are sites that are minimally disturbed with basal areas $>25 \mathrm{~m}^{2} /$ ha.

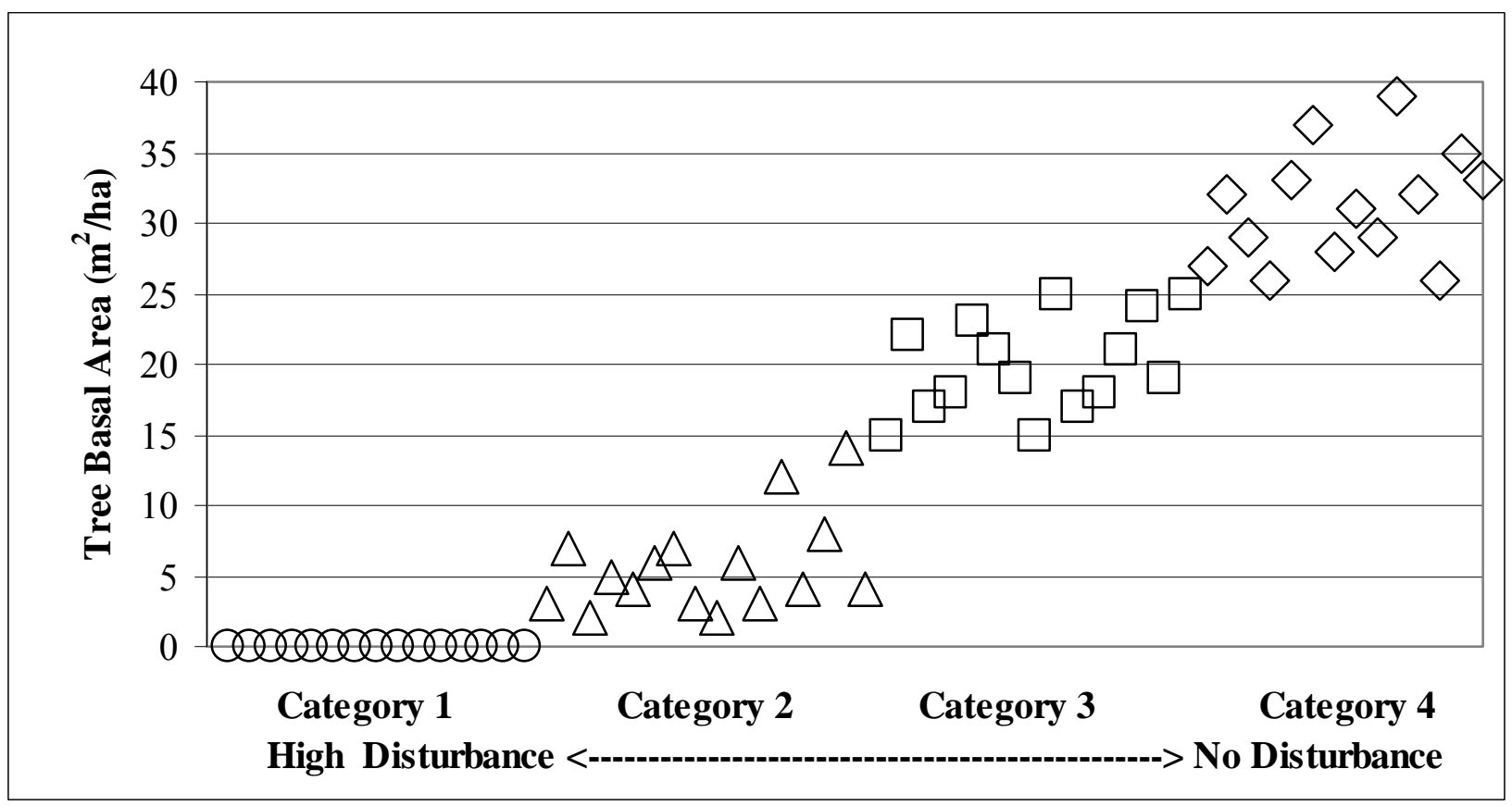

Figure 29. Tree basal area metric value in relation to disturbance categories.

\section{Correlation between variables}

Another approach to screening variables and metrics is to analyze the reference wetland data set for correlations that may indicate redundancies among variables. If two variables are highly correlated, it may be possible to eliminate one without significant loss of information. The simplest way to evaluate relationships among variables in the reference data set is to use a statistical program to calculate a correlation matrix. Separate correlation matrices should be calculated for the metric values and subindices for each variable. Figure 30 shows correlations among the metric values for four 


\begin{tabular}{|c|c|c|c|c|}
\hline SITE & RECUR & GRADIENT & MANNING & RATIO \\
\hline 1 & 5 & 0.02 & 0.05 & 25 \\
\hline 2 & 0 & 0.05 & 0.05 & 10 \\
\hline 3 & 8 & 0.12 & 0.12 & 15 \\
\hline 4 & 2 & 0.08 & 0.08 & 75 \\
\hline 5 & 1 & 0.01 & 0.02 & 22 \\
\hline 6 & 18 & 0.05 & 0.05 & 4 \\
\hline 7 & 6 & 0.09 & 0.1 & 5 \\
\hline 8 & 10 & 0.15 & 0.03 & 40 \\
\hline 9 & 0 & 0.02 & 0.11 & 20 \\
\hline 10 & 1 & 0.01 & 0.04 & 9 \\
\hline 11 & 2 & 0.05 & 0.06 & 12 \\
\hline 12 & 5 & 0.01 & 0.09 & 18 \\
\hline 13 & 12 & 0.08 & 0.03 & 80 \\
\hline 14 & 8 & 0.2 & 0.17 & 12 \\
\hline 15 & 3 & 0.07 & 0.13 & 5 \\
\hline & RECUR & GRADIENT & MANNING & RATIO \\
\hline RECUR & 1 & & & \\
\hline GRADIENT & 0.41 & 1 & & \\
\hline MANNING & -0.06 & 0.48 & 1 & \\
\hline RATIO & 0.12 & 0.13 & -0.32 & 1 \\
\hline
\end{tabular}

Figure 30. Metric values and correlation matrix for the variables $\mathrm{V}_{\text {FREQ }}$, $\mathrm{V}_{\text {SLOPE, }}$ and $\mathrm{V}_{\text {WIDTH. }}$

variables including $\mathrm{V}_{\text {FREQ }}, \mathrm{V}_{\text {SLOPE}}, \mathrm{V}_{\text {WIDTH}}$, and $\mathrm{V}_{\text {WIDTH }}$ with their respective metrics flood recurrence interval (years), gradient (feet per mile), Manning's n, and ratio of floodplain to channel width. Figure 31 gives the subindex values for the same four variables. The Pearson correlation coefficient ( $\mathrm{r}$ ) is provided at the bottom of the table. In the examples provided in Figures 30 and 31, the coefficients are relatively small (i.e., maximum $|r|=0.48$, or $r^{2}=0.23$ ). As a rule of thumb, one need not be concerned about redundancies between variables until the coefficient of determination $\left(\mathrm{r}^{2}\right)$ exceeds 0.50 (or $|\mathrm{r}|>0.70$ ), indicating that more than $50 \%$ of the variation in one measurement can be accounted for by changes in the other; $\mathrm{r}^{2}$ values exceeding $0.80(|\mathrm{r}| \geq 0.90)$ may indicate substantial redundancy between two measures.

If two variables are highly correlated, the assessment team should consider eliminating one of the variables from the assessment model. Factors to consider in deciding which of the two variables to keep include the ease of measurement, the accuracy and precision of the measurement, and the relevance of the variable to the anticipated wetland impacts in the region. 


\begin{tabular}{|c|c|c|c|c|}
\hline SITE & $V_{F R E Q}$ & $V_{S L O P E}$ & $V_{\text {ROUGH }}$ & $V_{\text {WIDTH }}$ \\
\hline 1 & 0.775 & 1 & 0.325 & 0.325 \\
\hline 2 & 0 & 1 & 0.325 & 0.1 \\
\hline 3 & 0.55 & 0.65 & 1 & 0.175 \\
\hline 4 & 1 & 0.85 & 0.6625 & 1 \\
\hline 5 & 1 & 1 & 0.1 & 0.28 \\
\hline 6 & 0.1 & 1 & 0.325 & 0.1 \\
\hline 7 & 0.7 & 0.8 & 0.8875 & 0.1 \\
\hline 8 & 0.4 & 0.5 & 0.1 & 0.55 \\
\hline 9 & 0 & 1 & 1 & 0.25 \\
\hline 10 & 1 & 1 & 0.2125 & 0.1 \\
\hline 11 & 1 & 1 & 0.4375 & 0.13 \\
\hline 12 & 0.775 & 1 & 0.775 & 0.22 \\
\hline 13 & 0.25 & 0.85 & 0.1 & 1 \\
\hline 14 & 0.55 & 0.25 & 0.6 & 0.13 \\
\hline 15 & 0.925 & 0.9 & 1 & 0.1 \\
\hline & $V_{F R E Q}$ & $V_{S L O P E}$ & $V_{\text {ROUGH }}$ & $V_{\text {WIDTH }}$ \\
\hline$V_{F R E Q}$ & 1 & & & \\
\hline$V_{S L O P E}$ & 0.08 & 1 & & \\
\hline$V_{\text {ROUGH }}$ & 0.04 & -0.08 & 1 & \\
\hline$V_{\text {WIDTH }}$ & 0.00 & -0.10 & -0.29 & 1 \\
\hline
\end{tabular}

Figure 31. Subindex values and correlation matrices for the model variables $\mathrm{V}_{\text {FreQ, }} \mathrm{V}_{\text {sLope, }} \mathrm{V}_{\mathrm{ROUGH}}$, and $\mathrm{V}_{\text {WIDTH. }}$

\section{Calibrate metric values}

When the relationship between a metric value and disturbance presents a clear trend or pattern, as is the case for the tree basal area data presented in Figure 29, assigning a subindex to metric values is a straightforward process. For example, in Figure 32 the range of metric values (26-39 $\left.\mathrm{m}^{2} / \mathrm{ha}\right)$ exhibited by reference standard wetlands (Category 4 in Figure 29) is assigned a subindex of 1.o. Highly disturbed, restorable sites are assigned a subindex of 0.1, and unrestorable sites are assigned a value of 0.o. Sites with low to moderate disturbance are assigned a subindex based on the linear relationship that exists between reference standard conditions and highly disturbed wetlands (Figure 33). This relationship will not always be linear, but may need to be fitted using a step (categorical), logarithmic, power, exponential, or other type of function. 


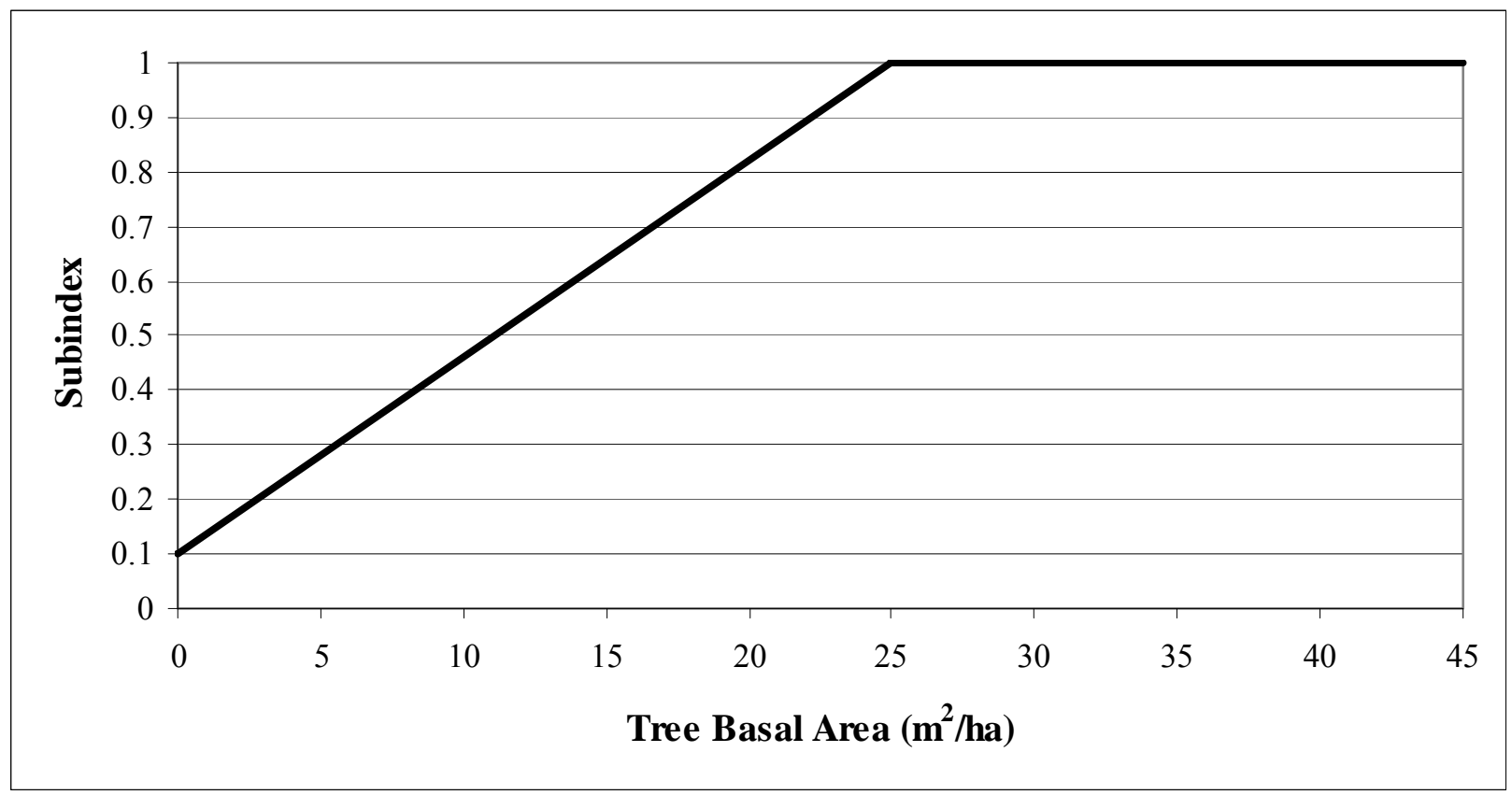

Figure 32. Subindex in relation to tree basal area metric values.

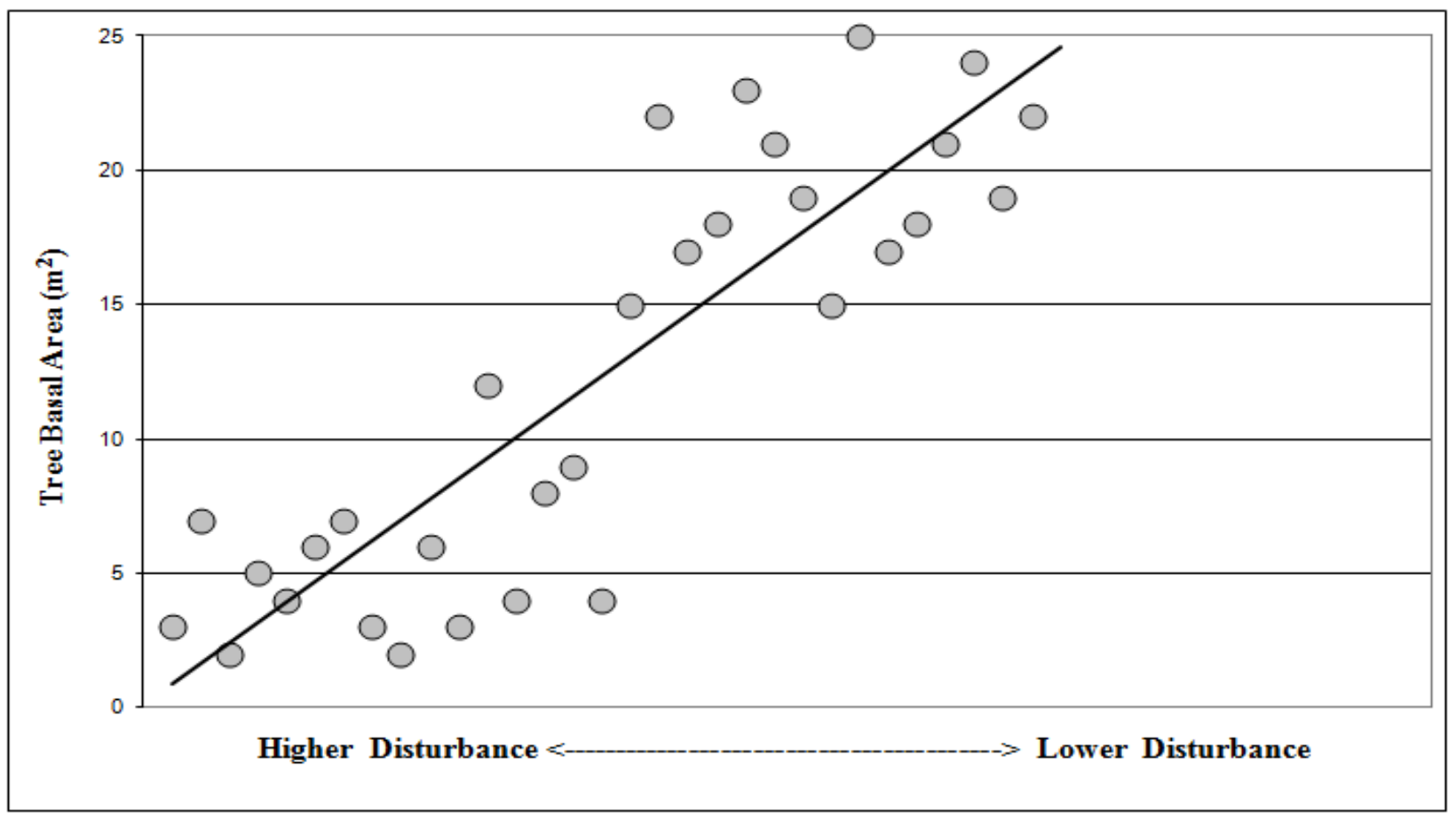

Figure 33. Regression of tree basal area against disturbance in moderately disturbed reference wetlands.

Notice in Figure 32 that when the metric value exceeds the range of values exhibited in reference standard wetlands (i.e., $>39 \mathrm{~m}^{2} / \mathrm{ha}$ ), a subindex of 1.0 is still assigned. This is because, in the specific case of the tree biomass variable, when the tree basal area metric values exceed the range of values exhibited in reference standard wetlands, it is interpreted as a wetland that 
supports an undisturbed, mature forest stand with large trees. Since this condition is within the range of metric values for reference standard wetlands, but was not included as a reference wetland, a subindex of 1.0 is still assigned. However, it is also possible that when metric values are greater than the conditions exhibited in reference standard wetlands, functional capacity is reduced. For example, in Figure 34 as the woody debris metric value increases or decreases from the range of metric values exhibited in reference standard wetlands, the subindex decreases. This is based on the interpretation that disturbance related to logging and other activities can initially lead to an increase in woody debris (i.e., slash) and subsequently to a reduction in woody debris because the source of the woody debris, trees, have been removed. Because an increase in woody debris is a temporary phenomenon with limited long-lasting effects, it only reduces functional capacity to a subindex of 0.5. Because the loss of trees has a long-lasting effect on woody debris, it can reduce functional capacity to a subindex of 0.1.

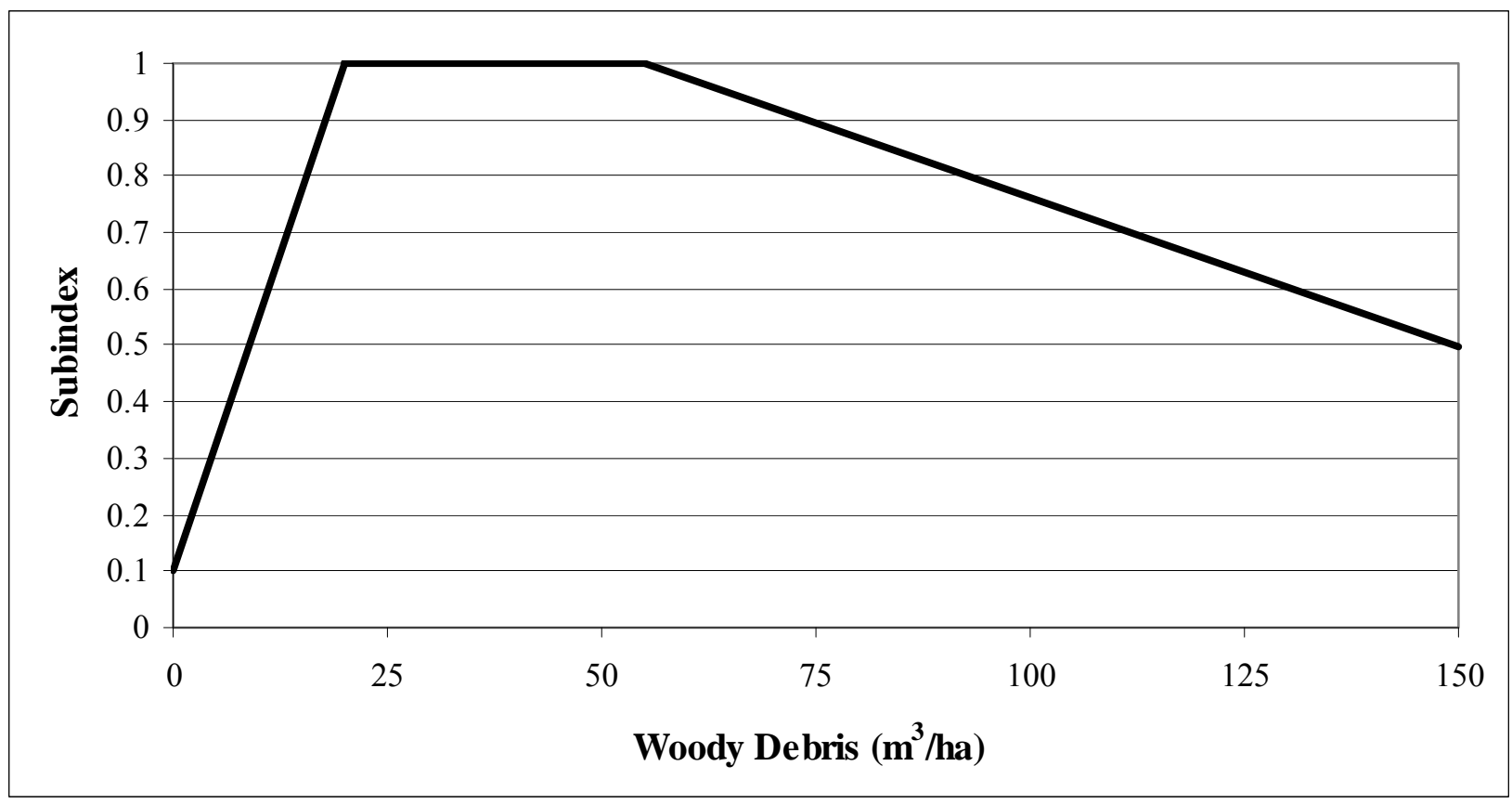

Figure 34. Subindices in relation to woody debris metric values.

The relationship between metric values and disturbances does not always present a clear pattern or trend, as shown in the scatter graph of metric values against disturbance gradient categories in Figure 28. While it is uncommon for the pattern to appear random, it is not uncommon for the pattern to be less clear than the example for tree basal area shown in Figure 29. There are a number of possible explanations for why the 
resulting pattern is unclear (Best and Stauffer 1986). First, it may be that the descriptions associated with the disturbance gradient rankings are too subjective and do not clearly identify how to assign rankings. Alternatively, it may be that the variable is simply not influenced by the types of disturbances represented by the disturbance gradient (i.e., the random pattern in Figure 28). A second possibility is that one or more portions of the disturbance gradient were inadequately sampled. A third possibility is that the variable was measured at different reference wetlands under different conditions (e.g., different seasons of the year, wet versus dry, or leaf-on versus leaf-off). A fourth possibility is that the variable was not measured accurately, or at a level of resolution capable of capturing the true response to disturbance. This could be due to sampling error (i.e., instrument or human) or an inadequate sampling design. Regardless of the reason, it is necessary to review the disturbance rankings assigned to the variable metric at each of the reference wetlands to determine which, if any, of the foregoing situations exist.

Based on this analysis, it may be necessary to reevaluate the way the disturbance gradient was defined, or the relationship between a metric value and the subindex. If this does not improve the patterns or trend between the metrics and the disturbance gradient, it may be necessary to drop the variable. A pilot study will often identify and resolve problems with specific variables, or disturbance gradient descriptions, before a fullscale collection of reference wetland data is undertaken.

Once the variables have been calibrated, the conceptual assessment models for each of the functions should be reviewed and updated to ensure that any changes that were made to variables during the calibration process are incorporated. This includes removing variables that have been dropped, adding new variables, and reviewing the way in which variables are combined in the equation to derive the FCI as discussed in Chapter 3, "Conceptualize Assessment Models."

\section{Verify assessment models}

Verifying assessment models is defined as a check of the logic and sensitivity of the model. The goal of verification is to answer the following types of questions. In general, does the model perform as envisioned by its developers? Is it sensitive to the types of project impacts that can be expected to occur to wetlands in the subclass? Do the variables in the model reflect the important attributes and processes that influence the 
function? Are all variables in the model actually needed or could the model be simplified without much loss of sensitivity? Is the mathematical equation used to combine variables appropriate? Are different variables given appropriate weight in the outcome? It should be noted that testing model accuracy is not the objective of verification. A model that has been verified may still be invalid in terms of accuracy or precision. Model accuracy is considered to be part of the process of validation, which is discussed in Chapter 7, "Validate Assessment Models."

To verify the logic of assessment models, one simply applies the model to real or hypothetical data and evaluates the results in light of one's experience and understanding of the wetland subclass. Verification is a fairly subjective procedure that is meant to determine whether model output makes sense, and should not be confused with model validation or testing for accuracy (Schroeder and Haire 1993). Verification can be done at any stage of the model development process. The two basic approaches to testing model logic include sensitivity analysis and applying the model to sample data sets.

\section{Sensitivity analysis}

Sensitivity analysis is an appraisal of model performance under incremental change in the input variables (Waide and Webster 1976, Overton 1977). Sensitivity analysis helps verify that the model will behave as intended under both moderate and extreme levels of each variable (Schroeder and Haire 1993). An important goal of sensitivity analysis is to identify the variables in the model with the most influence on the FCI, and, conversely, those variables that have little influence on the FCI. Variables that do not affect the FCI appreciably should be considered for elimination from the assessment model as a way of reducing sampling effort and enhancing the role of the remaining variables in the model. Alternatively, the assessment team may wish to develop more accurate sampling methods for highly influential variables while relying on more qualitative field methods for the less influential variables.

Sensitivity analyses of assessment models are accomplished by using the full range of subindex values and then examining the effects on the FCI. This type of analysis is used to verify that the equation is working as intended, that subindices for each variable are weighted properly, that FCI values are in the proper range (o to 1), and to determine the degree of influence each variable has on the FCI. Sensitivity analysis does not verify 
that the relationships between metric values and subindices are appropriate, nor does it verify that the model responds as intended to realistic levels of the environmental measurements. Therefore, additional checks are needed to fully verify model logic (see "Apply Assessment Results" in Chapter 6).

The easiest way to perform a sensitivity analysis of an assessment model is to enter the equation into a spreadsheet and incrementally vary the inputs to the model, one variable at a time. Effects on FCI can be examined directly from the spreadsheet, or simple statistics (e.g., means, ranges) can be used to quantify the influence of each variable on FCI predictions. More advanced applications use the software's graphing capabilities to plot changes in FCI under different combinations of subindex values.

Figure 35 presents a simple sensitivity analysis of a hypothetical threevariable model for the carbon-export function of a riverine wetland. The variables are flood frequency $\left(\mathrm{V}_{\mathrm{FREQ}}\right)$ and abundances of leaf litter $\left(\mathrm{V}_{\mathrm{LITTER}}\right)$ and coarse woody debris $\left(\mathrm{V}_{\mathrm{CWD}}\right)$. The assessment model incorporates the variables as FCI $=\left(\left(\mathrm{V}_{\mathrm{FREQ}}+\left(\mathrm{V}_{\mathrm{LITTR}}+\mathrm{V}_{\mathrm{CWD}}\right) / 2\right) / 2\right)^{1 / 2}$. Simple spreadsheet manipulations result in the calculation of FCI values for the three variables at intervals equal to 0.0, 0.1, 0.5, and 1.o. For more complex models, more intervals can be examined (e.g., o.0, 0.1, 0.2, 0.3, 0.4, etc). Some characteristics of the model are immediately obvious. First, whenever the subindex for $\mathrm{V}_{\mathrm{FREQ}}=\mathrm{O}$, the model always returns an $\mathrm{FCI}=0$. However, when both $\mathrm{V}_{\mathrm{LITTER}}=\mathrm{O}$ and $\mathrm{V}_{\mathrm{CWD}}=\mathrm{O}$, FCI values may range from $\mathrm{o}$ to 0.71 . Therefore, $V_{\text {FREQ }}$ has a controlling influence over model output. This form of model may be appropriate if the wetland function simply cannot occur in the absence of some important environmental feature or process (e.g., carbon export cannot occur when flood frequency is zero).

Other characteristics of the model shown in the spreadsheet (Figure 35) include the fact that $\mathrm{FCI}=0.71$ when all subindices are set to 0.5 , and that $\mathrm{FCI}=1.0$ only when all the subindices equal 1.0. The assessment team should decide whether the model behaves as intended, and use of the spreadsheet easily permits other equations to be tested until the intended model behavior is achieved. For example, the assessment team may believe that middle-of-the-road variable values (e.g., 0.5) for all three variables should depress FCI below 0.71. One option to achieve this result is to remove the exponent from the aggregation equation, resulting in $\mathrm{FCI}=0.5$. 


\begin{tabular}{|r|r|r|r|r|r|r|r|r|r|r|r|}
\hline \multicolumn{4}{|c|}{ Varying } & \multicolumn{4}{|c|}{ Varying $V_{\text {LITTER }}$} & \multicolumn{4}{|c|}{ Varying $V_{\text {CWD }}$} \\
\hline$V_{\text {FREQ }}$ & V $_{\text {LITTER }}$ & $V_{\text {CWD }}$ & FCI & $V_{\text {FREQ }}$ & $V_{\text {LITTER }}$ & $V_{\text {CWD }}$ & FCI & $V_{\text {FREQ }}$ & $V_{\text {LITTER }}$ & $V_{\text {CWD }}$ & FCI \\
\hline 0 & 0 & 0 & 0.00 & 0 & 0 & 0 & 0.00 & 0 & 0 & 0 & 0.00 \\
\hline 0.1 & 0 & 0 & 0.22 & 0 & 0.1 & 0 & 0.16 & 0 & 0 & 0.1 & 0.16 \\
\hline 0.5 & 0 & 0 & 0.50 & 0 & 0.5 & 0 & 0.35 & 0 & 0 & 0.5 & 0.35 \\
\hline 1 & 0 & 0 & 0.71 & 0 & 1 & 0 & 0.50 & 0 & 0 & 1 & 0.50 \\
\hline 0 & 0.1 & 0.1 & 0.22 & 0.1 & 0 & 0.1 & 0.27 & 0.1 & 0.1 & 0 & 0.27 \\
\hline 0.1 & 0.1 & 0.1 & 0.32 & 0.1 & 0.1 & 0.1 & 0.32 & 0.1 & 0.1 & 0.1 & 0.32 \\
\hline 0.5 & 0.1 & 0.1 & 0.55 & 0.1 & 0.5 & 0.1 & 0.45 & 0.1 & 0.1 & 0.5 & 0.45 \\
\hline 1 & 0.1 & 0.1 & 0.74 & 0.1 & 1 & 0.1 & 0.57 & 0.1 & 0.1 & 1 & 0.57 \\
\hline 0 & 0.5 & 0.5 & 0.50 & 0.5 & 0 & 0.5 & 0.61 & 0.5 & 0.5 & 0 & 0.61 \\
\hline 0.1 & 0.5 & 0.5 & 0.55 & 0.5 & 0.1 & 0.5 & 0.63 & 0.5 & 0.5 & 0.1 & 0.63 \\
\hline 0.5 & 0.5 & 0.5 & 0.71 & 0.5 & 0.5 & 0.5 & 0.71 & 0.5 & 0.5 & 0.5 & 0.71 \\
\hline 1 & 0.5 & 0.5 & 0.87 & 0.5 & 1 & 0.5 & 0.79 & 0.5 & 0.5 & 1 & 0.79 \\
\hline 0 & 1 & 1 & 0.71 & 1 & 0 & 1 & 0.87 & 1 & 1 & 0 & 0.87 \\
\hline 0.1 & 1 & 1 & 0.74 & 1 & 0.1 & 1 & 0.88 & 1 & 1 & 0.1 & 0.88 \\
\hline 0.5 & 1 & 1 & 0.87 & 1 & 0.5 & 1 & 0.94 & 1 & 1 & 0.5 & 0.94 \\
\hline 1 & 1 & 1 & 1.00 & 1 & 1 & 1 & 1.00 & 1 & 1 & 1 & 1.00 \\
\hline
\end{tabular}

Figure 35. Sensitivity analysis for the assessment model FCI $=\left(\left(V_{F R E Q}+\left(V_{\text {LITTER }}+V_{C W D}\right) / 2\right) / 2\right)^{1 / 2}$.

For a complicated model, it may be difficult to interpret model behavior from tabular spreadsheet output alone. Summary statistics and plots of model output are needed. Figure 36 presents a sensitivity analysis for the same three-model variable introduced above, in which summary statistics (e.g., range, minimum and maximum values) and graphical representation of results are examined. Graphical representations are generated for each model variable by plotting the FCI values presented in Figure 35 on an interval basis.

Figure 36A indicates that $V_{F R E Q}$ maintains a higher impact on FCI scores. This effect is seen in the higher initial response observed at low subindex values, as well as the steeper slopes on the FCI curves associated with $V_{\text {FREQ. }}$ Also, the identical graphs generated for $\mathrm{V}_{\text {LITTER }}$ and $\mathrm{V}_{\text {CWD }}$ demonstrate the equal treatment those variables receive within the assessment model (Figure 36B). In some cases sensitivity analysis can be used to justify the removal of repetitive or unnecessary variables. However, within the example model introduced here, $\mathrm{V}_{\text {LITTER }}$ and $\mathrm{V}_{\text {CWD }}$ work in concert to produce appropriate FCI scores. For example, recently restored areas may contain high levels of leaf litter available to contribute to carbon export functions, while lacking the long-term carbon stocks represented by coarse woody debris. Both variables must be present in the appropriate range for 


\begin{tabular}{|c|c|c|c|c|c|c|}
\hline Range & cor & aina & ased & ensi & anal & \\
\hline & & & $\mathrm{b}$ index $\mathrm{v}$ & lues & & \\
\hline Variable & & 0 & & & 0.1 & \\
\hline & Range & Low & High & Range & Low & High \\
\hline $\mathrm{V}_{\mathrm{FREQ}}$ & 0.71 & 0.00 & 0.71 & 0.52 & 0.22 & 0.74 \\
\hline $\mathrm{V}_{\text {LITTER }}$ & 0.50 & 0.00 & 0.50 & 0.30 & 0.27 & 0.57 \\
\hline $\mathrm{V}_{\mathrm{CWD}}$ & 0.50 & 0.00 & 0.50 & 0.30 & 0.27 & 0.57 \\
\hline & & & index $v$ & lues & & \\
\hline Variable & & 0.5 & & & 1.0 & \\
\hline & Range & Low & High & Range & Low & High \\
\hline$V_{\text {FREQ }}$ & 0.37 & 0.50 & 0.87 & 0.29 & 0.71 & 1.00 \\
\hline $\mathrm{V}_{\text {LITTER }}$ & 0.18 & 0.61 & 0.79 & 0.13 & 0.87 & 1.00 \\
\hline $\mathrm{V}_{\mathrm{CWD}}$ & 0.18 & 0.61 & 0.79 & 0.13 & 0.87 & 1.00 \\
\hline
\end{tabular}

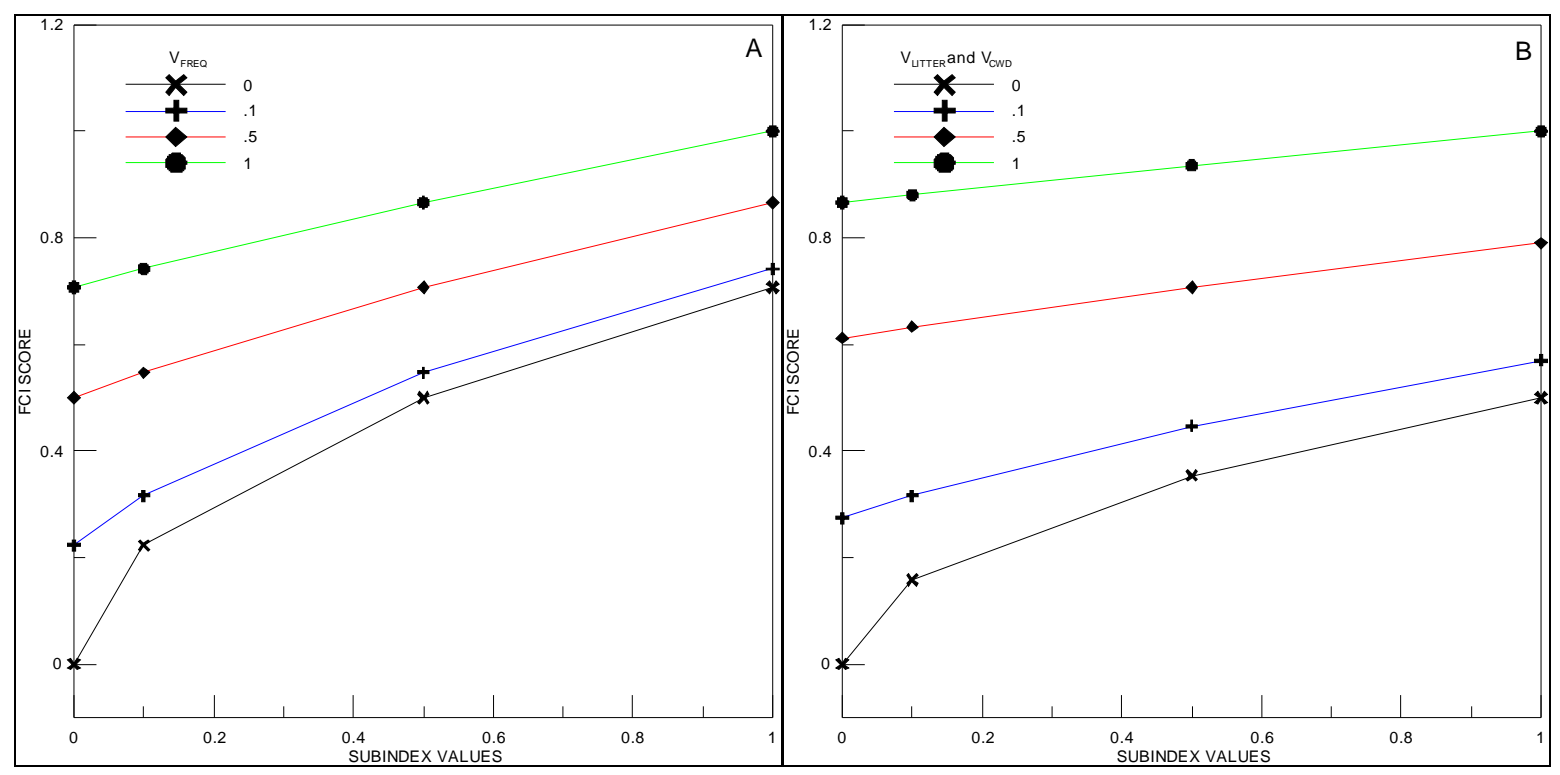

Figure 36. Tabular and graphical display of sensitivity results for the simple three-variable assessment model $\mathrm{FCl}=\left(\left(V_{F R E Q}+\left(V_{L I T E R}+V_{C W D}\right) / 2\right) / 2\right)^{1 / 2}$. The higher initial response and increased slope observed for $V_{F R E Q}(A)$ indicate that the variable maintains a stronger impact on $\mathrm{FCl}$ scores compared to $V_{\text {LITTER }}+$ $V_{C W D}(B)$. Note that $V_{L I T T R}$ and $V_{C W D}$ receive equal treatment in the model, resulting in identical graphs.

the function to generate FCI scores $=1$.o. Within the current example, all three variables respond to changes in subindex values across the sample interval examined.

Care should be taken in conducting sensitivity and developing spreadsheets. For example, models may specify a different potential range for certain variables. In the western Kentucky, low-gradient riverine model (Ainslie et al. 1999), for instance, VSLOPE only takes values from 0.1 to 1.0; zero is not an 
appropriate value. Therefore, to investigate the effect of $\mathrm{V}_{\text {SLOPE }}$ on model outcome, only subindex values from 0.1 to 1 should be considered.

\section{Test assessment models with sample data sets}

As mentioned previously, a sensitivity analysis of variable subindices cannot verify that the model will respond appropriately to actual values of the field measurements. This can only be done by inputting the actual metric values for each variable and examining both the resulting subindices and the FCI. Appropriate data sets for such an analysis may already be available for wetland sites used in the calibration phase and additional sites representing a range of conditions can be sampled specifically for this purpose. Another option is to generate a hypothetical data set of realistic values for each variable.

Application of the model to data from a small number (e.g., 10 to 20) of wetland sites can readily be done by hand calculation. Larger applications can be facilitated by programming the complete assessment model (including transformation of metric values to subindices) into a spreadsheet and inserting either real or hypothetical field data into the appropriate cells. Another option is to use a statistical package with programming capabilities to write the model and run the test data.

The output of a successfully tested model should demonstrate that the model produces variable subindices and FCI values in the appropriate range (i.e., o to 1). The assessment team should next determine whether FCI values appear reasonable given the knowledge and experience of the assessment team. For example, if the FCI scores at high quality sites fail to exceed 0.80 , then the model may be scoring these wetlands too low.

Furthermore, if the test data set contains highly degraded sample sites and no wetlands exhibited FCI scores lower than 0.20, the assessment team may need to revisit model calibration and consider modifications based on their knowledge and experience.

\section{Field-test assessment models}

The objective of field-testing is to ensure that end-users can apply the assessment model efficiently and with consistent results. One of the goals of the HGM Approach is to develop end user assessment protocols that are capable of assessing wetland functions rapidly, within the time and other constraints imposed by regulatory programs. Field testing is employed to 
determine how long it takes to apply the assessment protocols under typical field situations, identify incomplete or ambiguous instructions, and ensure that the level of training and expertise required to apply the assessment protocols are appropriate. In addition, field-testing should verify that the model can be used consistently year-round (if that is what the authors intended) and that different end users applying the assessment protocols in the same wetland will arrive at the same results.

Field-testing helps to ensure that a model can be applied quickly and efficiently, and that the results are consistent and reproducible, at least within limits acceptable to the assessment team. As with verification, model accuracy is not the concern. There are no firm guidelines concerning how long it should take to apply an assessment model to a typical field site, nor how consistent results must be from one investigator to the next. Both depend upon the constraints and expectations of the end user. The assessment team should establish and document realistic goals for time and repeatability in advance of any field-testing. For routine regulatory purposes, application of the set of assessment models for all the functions performed by a wetland of a particular subclass should probably take no more than a few hours. Requirements for consistency are dictated by the intended use of the model. An assessment model that is used to guide multimillion-dollar land-use decisions should be tested to a higher standard than one intended solely for routine wetland management or advancedidentification projects.

An important issue in model consistency is the inherent variability of many quantitative measures across a wetland site and the statistical considerations of sample size and sampling design. Sampling procedures recommended in a guidebook should be based, in part, on analysis of data from reference wetlands. Recommended sample sizes (e.g., number of plots or transects) are a trade-off between the desire for a rapid assessment and the need for confidence in the estimates of each variable and FCI.

The following section describes a generic procedure for field-testing assessment models. The procedure is adaptable to different levels of effort in data gathering and analysis, depending on the needs of and constraints upon the assessment team. A relatively simple test might involve only a small number of participants (e.g., 6 to 10) and a few field sites. Larger samples of test participants (e.g., 25 or more) may be required to determine the distribution of metric values and FCIs, and to give the 
assessment team more confidence that different investigators assessing the same site will obtain similar results. Berkowitz et al. (2011) describe field testing of a specific HGM model.

\section{Generic procedure for field-testing assessment models}

Table 16 lists the steps involved in a field test of a draft assessment model. This procedure can be used to test the model for a single function or the set of functional models performed by a wetland subclass. Models for different functions often use some of the same variables; therefore, a realistic evaluation of the amount of time required to apply the set of models for that subclass is possible only if the models for all functions are applied at once.

Table 16. Sequence of steps in a generic field-test procedure.

1. Identify a number of individuals to serve as field testers. The larger the sample of testers, the more reliable the conclusions about the distribution of model scores.

2. Select at least three to five wetland field sites representing a range of conditions relative to reference standards.

3. Provide the draft guidebook (including models, instructions, and data forms) and background site information to testers in advance of site visits.

4. Schedule site visits by each tester independently, if possible. In any case, testers should not be influenced by other test participants. Consider scheduling two or more rounds of tests to evaluate seasonal or annual bias.

5. Ask testers to record the amount of time required to apply the model at each field site and, after completion of all field visits, to provide a written critique of the model's instructions, sampling procedures, and calculations.

6. Combine field results from all testers. Evaluate consistency of $\mathrm{FCl}$ scores across testers for each wetland function considered.

7. If model output is inconsistent, modify the model, instructions, or sampling recommendations to reduce variability. If necessary, schedule a new field test to ensure that any modifications achieved the intended results.

The first step in field-testing the models is to identify a number of individuals willing to serve as testers. The assessment team should choose people who were not involved in the development of the models, sampling protocols, or the instructions for their use. It is important to select individuals whose training and experience are similar to those of anticipated end users of the models (e.g., regulatory personnel, private consultants, resource managers). All participants should have experience with basic methods for sampling environmental characteristics.

Next, select a manageable number of wetland field sites (the authors suggest at least three to five sites) in the reference domain of the subclass. 
Include at least one site that represents reference standard conditions and two or more that deviate from reference standard conditions. Some of the same reference wetland sites used for model calibration may be adequate for this purpose; it is not necessary to select new sites. To test consistency of model output, it is more important to maximize the number of testers than it is to increase the number of sites. A field test involving 20 people and three field sites is likely to provide more useful data than one involving only six people and 10 sites.

Each model tester should be provided in advance with the models, field data forms, sampling protocols, and detailed instructions for their use. In addition, background information on the field sites should be provided, including topographic maps, soil survey information, National Wetlands Inventory maps, hydrology data, and any other off-site data required by the assessment procedure. Testers should be thoroughly familiar with the instructions for using the models before they go to the field.

It is important for each individual tester to provide an independent determination of FCI for a site that is unbiased. The preferred option is to schedule separate site visits by each model tester, if possible. If separate visits are not practical, take steps to ensure that participants do not interact, cooperate, or interfere with each other during the tests.

Two potential goals of field-testing are (1) to evaluate the clarity of instructions for applying the guidebook by assessing the consistency of results across different individuals, and (2) to evaluate seasonal or annual variations in FCI scores produced by the model. All draft assessment models should be evaluated for investigator consistency (goal 1). To do this, all field testers should be scheduled for site visits within a 1- to 2-week period to minimize the influence of temporal changes in site conditions on FCI scores. In addition, any model that contains variables whose interpretation might change seasonally (e.g., spring versus summer) or annually (e.g., wet versus dry years) should also be evaluated for temporal consistency (goal 2). This can be done by scheduling two or more rounds of field tests during different seasons or years (see "Evaluate Temporal Consistency" section below).

Upon arrival at a field site, testers should be oriented relative to site maps and important landmarks, made aware of the boundaries of the wetland assessment area, provided with any necessary tools, and then asked to perform the assessment. Each tester should record the amount of time 
required to gather field data at each site, and should use his or her data to determine subindices for each variable and FCI values for each function. When sampling and data analysis are completed at all field sites, testers should be asked to provide written comments addressing the clarity, completeness, and "user friendliness" of the instructions for applying the models, sampling procedures, and calculations. A form such as that shown in Figure 37 may be used to record the testers' comments.

Functional capacity indices for each function at each field site are then compiled and compared to evaluate consistency in scoring by different testers. As mentioned previously, there are no established standards for consistency of model outputs across investigators and the desired precision may vary with the goals of the application (e.g., general resource inventories versus high-value impact analyses). Therefore, the assessment team should establish goals for investigator consistency in advance of field-testing. For most regulatory uses, including wetland impact assessments, project alternatives analyses, and calculation of mitigation requirements, an appropriate test goal is that $90 \%$ of users who apply a model in the same assessment area should produce an FCI score that is within 0.15 of the median score for all users combined.

As an example, Table 17 presents the results of a simple field test involving six participants who were asked to apply a set of five functional assessment models to a series of sites. Results for only one field site are shown. Due to the small number of testers involved, analysis of these data is necessarily subjective and consistency standards should be applied with flexibility. FCI scores for Functions 1, 3, and 5 clearly meet the goal in that all six participants in the test achieved scores within 0.15 of the median score for each function. Results for Function 4 (Table 17) are very consistent (5 of 6, or $83 \%$, achieved the same score) with the exception of that obtained by Tester E. Examination of the tester's written comments is valuable in reconciling outlying scores. In this case, the low score by Tester E may reflect his confusion over some part of the instructions that could be corrected easily. The fact that other testers gave consistent scores may indicate that the instructions and model documentation are basically sound.

Model consistency should be evaluated across field sites representing reference standard conditions and the gradient of disturbances commonly encountered within the reference domain. This should include undisturbed, moderately disturbed, and highly disturbed areas. Inconsistencies may be more obvious and informative at sites having intermediate levels of function than at sites representing the extremes. For example, the perfect 


\begin{tabular}{|c|c|c|c|c|c|}
\hline \multicolumn{6}{|c|}{ HGM Assessment Model Field Tester's Evaluation Form } \\
\hline \multicolumn{4}{|c|}{ Tester's Name: name } & \multicolumn{2}{|c|}{ Assessment Model: Headwater Slope } \\
\hline \multicolumn{2}{|c|}{ Phone: phone \# } & \multicolumn{3}{|c|}{\begin{tabular}{|l|l|} 
Date: $6 / 22 / 2008$ & E-Mail: \\
\end{tabular}} & \\
\hline \multicolumn{6}{|c|}{ Time Required to Apply Model(s) } \\
\hline $\begin{array}{l}\text { Field Site } \\
1\end{array}$ & \multicolumn{2}{|c|}{ Start time: 0800} & \multicolumn{2}{|c|}{ Finish time: 1045} & $\begin{array}{l}\text { Total time: } 2 \mathrm{hr} 45 \\
\text { min }\end{array}$ \\
\hline $\begin{array}{l}\text { Field Site } \\
2\end{array}$ & \multicolumn{2}{|c|}{ Start time: 1300} & \multicolumn{2}{|c|}{ Finish time: 1500} & Total time: $2 \mathrm{hr}$ \\
\hline $\begin{array}{l}\text { Field Site } \\
3\end{array}$ & \multicolumn{2}{|c|}{ Start time: 1530} & \multicolumn{2}{|c|}{ Finish time: 1630} & Total time: $1 \mathrm{hr}$ \\
\hline $\begin{array}{l}\text { Field Site } \\
4\end{array}$ & \multicolumn{2}{|c|}{ Start time: } & \multicolumn{2}{|c|}{ Finish time: } & Total time: \\
\hline $\begin{array}{l}\text { Field Site } \\
5\end{array}$ & \multicolumn{2}{|c|}{ Start time: } & \multicolumn{2}{|c|}{ Finish time: } & Total time: \\
\hline \multicolumn{6}{|c|}{$\begin{array}{l}\text { To apply the model(s), did you need any documents or tools that were not } \\
\text { available? Please list: }\end{array}$} \\
\hline \multicolumn{6}{|c|}{ No - all documents and tools were readily accessible. } \\
\hline \multicolumn{6}{|c|}{$\begin{array}{l}\text { Did application of the model(s) require training or experience that you lacked? } \\
\text { Please list: }\end{array}$} \\
\hline \multicolumn{6}{|c|}{ Plant Identification } \\
\hline \multicolumn{6}{|c|}{$\begin{array}{l}\text { Were the instructions clearly written and easy to follow? Identify specific } \\
\text { problems or ambiguities: }\end{array}$} \\
\hline \multicolumn{6}{|c|}{$\begin{array}{l}\text { The instructions were not clear on determining the number of plots necessary to } \\
\text { complete the assessment. }\end{array}$} \\
\hline \multicolumn{6}{|c|}{$\begin{array}{l}\text { Describe any general problems you had in determining subindex levels for each } \\
\text { variable. }\end{array}$} \\
\hline \multicolumn{6}{|c|}{ No problems were encountered } \\
\hline \multicolumn{6}{|c|}{ Describe any general problems you encountered with calculation of FCI values. } \\
\hline \multicolumn{6}{|c|}{ No problems were encountered } \\
\hline
\end{tabular}

Figure 37. Example field-tester evaluation form. 
HGM Assessment Model Field Tester's Evaluation Form (continued)

For each variable listed below, give your opinion as to (1) the clarity of the instructions for measuring that variable in the field, (2) ease of making the field measurement, and (3) whether conversion of the measure to a subindex was clear and straightforward. Use the following scale for your response: 1-strongly disagree, 2-disagree, 3-no opinion, 4-agree, 5-strongly agree

\begin{tabular}{|l|c|c|c|}
\hline Variable & $\begin{array}{c}\text { Sampling } \\
\text { instructions } \\
\text { were clear }\end{array}$ & $\begin{array}{c}\text { Field measurement } \\
\text { was easy }\end{array}$ & $\begin{array}{c}\text { Conversion to } \\
\text { subindex was } \\
\text { straightforward }\end{array}$ \\
\hline$V_{A}$ & 5 & 5 & 4 \\
\hline$V_{B}$ & 5 & 5 & 4 \\
\hline$V_{C}$ & 4 & 2 & 4 \\
\hline$V_{D}$ & 5 & 5 & 4 \\
\hline$V_{E}$ & 4 & 1 & \\
\hline etc. & & & 3 \\
\hline
\end{tabular}

For each function listed below, give your opinion (1) whether calculation of the FCI was clear, and (2) whether the FCI agreed with your subjective opinion of the quality of the site(s) for that function. Explain any differences of opinion. Use the scale given above for your responses.

\begin{tabular}{l|c|c|} 
Function & $\begin{array}{c}\text { FCI calculation } \\
\text { was clear }\end{array}$ & $\begin{array}{c}\text { FCI agreed with my } \\
\text { subjective judgement }\end{array}$ \\
\hline Function \#1 & 5 & 5 \\
\hline Function \#2 & 5 & 4 \\
\hline Function \#3 & 4 & 3 \\
\hline etc. & $\begin{array}{l}\text { Do you think that the instructions for using this model in the field are ready for } \\
\text { publication and distribution? If not (and not covered above), please describe what } \\
\text { needs to be done: }\end{array}$ \\
Guidebook is ready for publication with minor edits and clarification
\end{tabular}

Figure 37. (continued).

consistency among users of the model for Function 5 (Table 17) at that site may be due to some obvious limitation (e.g., the function requires surface flow and the site never floods); this does not mean that model outcome would be consistent among users on a site that does flood.

Scores for Function 2 (Table 17) are highly variable. The model for this function clearly fails to meet the stated goal for investigator consistency. The assessment team should consider revising the instructions, model calibration, or variable scaling of Function 2. 
Table 17. Comparison of field tester results at one field site.

\begin{tabular}{|l|l|l|l|l|l|}
\hline \multirow{2}{*}{ Tester } & \multicolumn{5}{|c|}{ FCI Scores } \\
\cline { 2 - 6 } & Function 1 & Function 2 & Function 3 & Function 4 & Function 5 \\
\hline A & 0.3 & 0.9 & 1.0 & 0.8 & 0.0 \\
\hline B & 0.25 & 0.6 & 1.0 & 0.8 & 0.0 \\
\hline C & 0.3 & 0.8 & 0.9 & 0.8 & 0.0 \\
\hline D & 0.2 & 0.3 & 1.0 & 0.8 & 0.0 \\
\hline E & 0.25 & 0.6 & 1.0 & 0.4 & 0.0 \\
\hline F & 0.3 & 0.5 & 1.0 & 0.8 & 0.0 \\
\hline & \multicolumn{7}{|c|}{ Scoring Summary } & \\
\hline Min & 0.2 & 0.3 & 0.9 & 0.4 & 0.0 \\
\hline Max & 0.3 & 0.9 & 1.0 & 0.8 & 0.0 \\
\hline Median & 0.275 & 0.6 & 1.0 & 0.8 & 0.0 \\
\hline
\end{tabular}

Figure 38 shows the distribution of FCI scores for a different field test involving a larger number of participants $(n=30)$ and models for two functions. Again, results for only one field site are shown. The larger sample size provides more information about the distribution of FCI scores than did the previous example. Applying the same goal for investigator consistency, the model for Function 1 passes the test (i.e., 28 of 30, or 93\%, of FCI scores fall within 0.15 of the median score for all test participants). Scores for Function 2, however, are too variable. Only 47\% (14 of 30) of FCI scores fall within the desired range (Figure 40).

There may be several reasons why a model would fail to meet goals for investigator consistency, including (1) unclear definitions of model variables, (2) use of low-resolution or error-prone sampling methods, (3) unclear instructions for data gathering, and (4) investigator errors in calculating subindex and FCI values. In addition, if an assessment area is large or heterogeneous, sample sizes recommended in the Guidebook may not be large enough to achieve adequate precision in the estimates of quantitative variables (see Chapter 5). This problem can be corrected by requiring larger samples (e.g., more plots) at the expense of application speed.

Sometimes the problems with model consistency can be traced to only one or two variables. Written comments provided by model testers are valuable in identifying such problems and providing suggestions for model improve- 
ment. Another way to identify problem variables is to plot histograms of subindex scores, similar to the plots of FCI values shown in Figure 40. Model revisions should attempt to reduce the variability across the subindices that are calculated by different individuals.

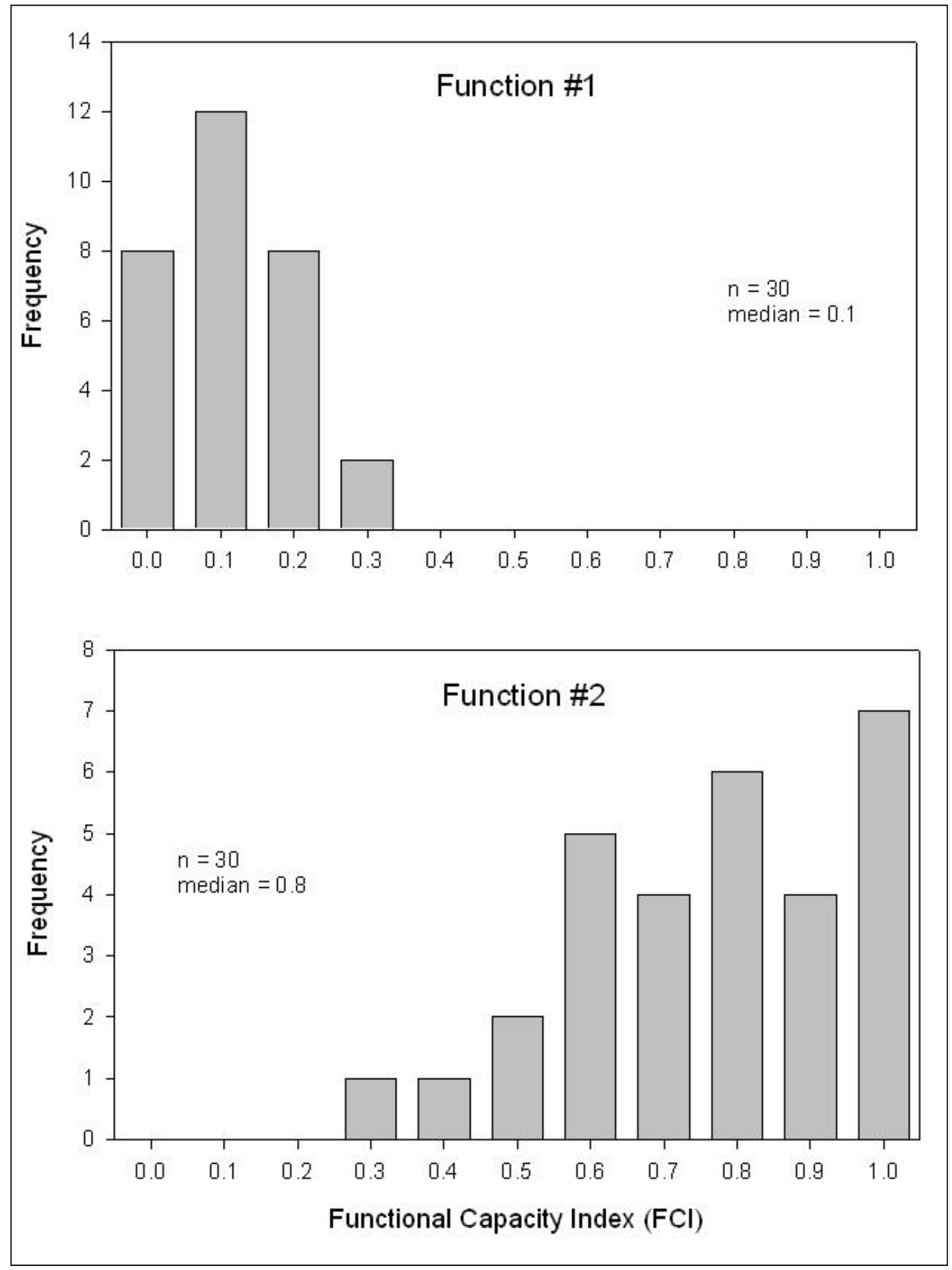

Figure 38. Results of field tests of assessment models for two functions at one field site. 
Assessment models that undergo extensive changes as a result of a field test should be tested again to determine whether the consistency of model scores across investigators has improved. For a repeat field test, some of the same and some different participants should be used.

\section{Evaluate temporal consistency}

Most assessment models are designed to be used throughout the year, at least when weather conditions are adequate for sampling (i.e., snow is not too deep and soils are unfrozen). Some assessment models may contain alternative variables to use when particular conditions exist (e.g., when surface water is present versus absent). However, the capacity of a wetland to perform a function does not change seasonally or annually in undisturbed situations. Therefore, a model applied to a particular wetland should give the same score regardless of when the investigation is done.

Any model that contains variables that may change seasonally or annually should be field tested for temporal consistency. This includes models whose variables may be more difficult to evaluate during certain periods, such as during dry seasons or years. Temporal consistency is evaluated by applying the model at different times and comparing the results.

Table 18 shows the results obtained by 12 field testers who applied the model for one function at one wetland site during spring and again in late summer. This example used the Mann-Whitney test, a nonparametric analogue to the t-test (Zar 1999), to determine whether distributions of FCI scores differed between sampling dates. The lack of a significant difference in FCI scores indicates that the model gives results that are consistent through the year.

In some cases, it may be possible to modify the model to improve temporal consistency without reducing potential model accuracy by emphasizing only the most stable environmental features or measurements. For example, a wildlife model might require an estimate of acorn availability as one variable affecting winter food supplies. A direct measurement of the abundance of fallen acorns would vary seasonally, especially in areas affected by flooding. A surrogate variable, such as density of acorn-producing trees, is temporally more stable and could be measured at any time of year.

In other cases, critical environmental measurements may be impossible to take at certain times of year (e.g., pH of surface water or maximum flow velocity when wetlands are dry). One option is to include less reliable 
indicator variables as alternatives if the assessment must be done at an inappropriate time. Model documentation should include the results of a consistency test (e.g., Table 18) and should state that model scores may be less reliable if an indicator variable must be substituted for the preferred measurement.

Table 18. Field test for temporal consistency of one function.

\begin{tabular}{|c|c|c|c|c|}
\hline \multirow[b]{3}{*}{ Tester } & \multicolumn{4}{|c|}{$\mathrm{FCl}$ Values for Function 1} \\
\hline & \multicolumn{2}{|c|}{$\begin{array}{l}\text { Spring Sampling } \\
\text { (25-30 April) }\end{array}$} & \multicolumn{2}{|c|}{$\begin{array}{l}\text { Late Summer Sampling } \\
\text { (1-15 August) }\end{array}$} \\
\hline & $\mathrm{FCl}$ & Rank & $\mathrm{FCl}$ & Rank \\
\hline A & 0.4 & 6 & 0.3 & 2 \\
\hline B & 0.5 & 13.5 & 0.5 & 13.5 \\
\hline C & 0.5 & 13.5 & 0.4 & 6 \\
\hline $\mathrm{D}$ & - & -- & 0.5 & 13.5 \\
\hline E & 0.6 & 20 & 0.5 & 13.5 \\
\hline D & 0.4 & 6 & 0.3 & 2 \\
\hline$E$ & 0.5 & 13.5 & -- & -- \\
\hline $\mathrm{F}$ & -- & - & 0.4 & 6 \\
\hline G & 0.3 & 2 & 0.5 & 13.5 \\
\hline $\mathrm{H}$ & 0.5 & 13.5 & 0.6 & 20 \\
\hline I & 0.4 & 6 & 0.6 & 20 \\
\hline$J$ & 0.5 & 3.5 & 0.5 & 13.5 \\
\hline \multicolumn{5}{|c|}{ Example two-tailed Mann-Whitney test with tied ranks (Zar 1984): } \\
\hline \multirow{2}{*}{ Sample sizes: } & \multicolumn{4}{|c|}{$n_{1}=10$} \\
\hline & \multicolumn{4}{|c|}{$\mathrm{n}_{2}=11$} \\
\hline \multirow{2}{*}{ Sum of ranks: } & \multicolumn{4}{|c|}{$\mathrm{R}_{1}=107.5$} \\
\hline & \multicolumn{4}{|c|}{$\mathrm{R}_{2}=123.5$} \\
\hline Test statistic: & \multicolumn{4}{|c|}{$U=(10)(11)+(10)(10+1) / 2-107.5=57.5$} \\
\hline
\end{tabular}




\section{Develop End-User Assessment Protocol}

\section{Introduction}

This chapter provides guidance on developing an assessment protocol for the collection and interpretation of data that is necessary to assess wetland functions using the HGM Approach. The assessment protocol is the part of the guidebook that end-users will use most frequently, and therefore, it is important that the assessment protocol provides clear instructions in a user-friendly format. This will ensure that the assessment protocol is applied accurately and consistently. The assessment team should review other guidebooks for examples of the level of specificity that is required for the assessment protocol. Examples representing different subclasses include: riverine (Ainslie et al. 1999), depressions (Hauer et al. 2002), flats (Noble et al. 2002), multiple subclasses (Klimas et al. 2004, 2005; Klimas 2006), headwater streams (Noble et al. 2007), and coastal wetlands (Shafer et al. 2007).

The remainder of this chapter discusses each of the following tasks required to develop the assessment protocol.

1. Define assessment objectives.

2. Characterize the project area.

3. Screen for red flags.

4. Define the Wetland Assessment Area.

5. Determine the wetland subclass.

6. Collect the data.

7. Analyze the data.

8. Apply assessment results.

\section{Define assessment objectives}

The first task to be addressed in the assessment process is to unambiguously identify the purpose of the assessment. Defining the purpose helps to clarify the approach that should be taken, and can be as simple as stating, "The purpose of this assessment is to determine how the proposed project will impact wetland functions." 
The most common assessment scenario is a comparison of the functional capacity of pre- and post-project conditions in the wetland assessment area (WAA). Data for the pre-project assessment are collected under existing conditions at the project site, while data for the post-project assessment are normally based on the conditions that are expected to exist following proposed project impacts.

Frequently, there are multiple purposes for conducting an assessment. The guidebook should assist the end-user in carefully defining the purpose(s) of the assessment to facilitate communication and understanding among the people involved in the assessment, and to make the purpose(s) of the assessment clear to other interested parties. Potential objectives include:

1. Compare several wetlands as part of an alternatives analysis.

2. Identify specific actions that can be taken to minimize project impacts.

3. Document baseline conditions at a wetland site.

4. Determine mitigation requirements.

5. Determine mitigation success.

6. Determine the effects of a wetland management technique

\section{Characterize the project area}

Characterizing the project area involves describing the physical and biological conditions in the Wetland Assessment Area (WAA), as well as proposed impacts that have the potential to influence how wetlands in the project area perform functions. An overview of general ecological information is typically presented for the entire reference domain in Chapter 3 of each guidebook, but a site-specific description should be assembled for each WAA within the project area. The guidebook should provide guidance to the user on how to characterize the WAA(s), including any maps and figures that are helpful in determining project area boundaries, jurisdictional wetlands, the boundaries of the WAA(s) (discussed later in this chapter), roads, ditches, buildings, streams, soil types, plant communities, threatened or endangered species habitat, and other important features. Some helpful sources of information are aerial photographs, topographic and National Wetland Inventory (NWI) maps, and county soil surveys. Most of the spatial data listed above can be most efficiently assembled and managed using a Geographic Information System (GIS).

In addition to characterizing the WAA, project impacts that ultimately affect wetland functions need to be identified for the proposed project. For example, impacts that directly influence the physical features of a wetland 
may include vegetation removal or placement of fill material. Any changes in wetland hydrology as a result of a proposed project also need to be determined. Changes in hydrology can be assessed by developing maps that show the frequency and duration of flooding for the preproject and postproject conditions. These maps can be used to determine if changes in hydrology would result in a change in wetland subclass, for example, that would result in losses or gains in functions. If more than one alternative for a project is being considered, these impacts can be compared in terms of the land area that would be affected.

\section{Screen for red flags}

Red flags are features within or in the vicinity of the project area to which special recognition or protection has been assigned. Many red flag features, such as those based on national criteria or programs, are similar from region to region. Other red flag features are based on regional or local criteria. The guidebook should provide the user with a list of potential red flag issues that should be evaluated prior to the assessment of wetland functions. Table 19 is a list of potential red flag features assembled for one particular region, but a guidebook should include any specific local or state regulations or special areas that may be appropriate. Screening for red flag features represents a proactive attempt to determine if the wetlands or other natural resources in and around the project area require special consideration or attention that may preempt or postpone the need for an assessment of wetland functions. An assessment of wetland functions may not be necessary if the project is likely to be stopped due to the potential impact to a threatened or endangered species or habitat, for example. An assessment of wetland functions may be unnecessary in this case since the project may be denied or modified strictly on the basis of the impacts to threatened or endangered species or habitat.

\section{Define the wetland assessment area}

The wetland assessment area (WAA) is an area of wetland within a project area that belongs to a single wetland subclass and is relatively homogeneous with respect to the site-specific criteria used to assess wetland functions (i.e., hydrologic regime, vegetation structure, topography, soils, successional stage, etc.). Figures 39-42 illustrate a variety of possible alternative WAA designations and arrangements. In many project areas, there will only be one WAA representing a single wetland subclass (Figure 39). However, as the size and heterogeneity of the project area increase, it may be necessary to define and assess multiple WAAs within the project area. 
Table 19. Red flag features and respective program or agency authority.

\begin{tabular}{|l|l|}
\hline Red Flag Features & Authority ${ }^{1}$ \\
\hline Native Lands and areas protected under American Indian Religious Freedom Act & A \\
\hline Hazardous waste sites identified under CERCLA or RCRA & I \\
\hline Areas protected by a Coastal Zone Management Plan & E \\
\hline Areas providing Critical Habitat for Species of Special Concern & B, C, F \\
\hline Areas covered under the Farmland Protection Act & K \\
\hline Floodplains, floodways, or floodprone areas & J \\
\hline Areas with structures/artifacts of historic or archeological significance & G \\
\hline Areas protected under the Land and Water Conservation Fund Act & K \\
\hline Areas protected by the Marine Protection Research and Sanctuaries Act & B, D \\
\hline National wildlife refuges and special management areas & C \\
\hline Areas identified in the North American Waterfowl Management Plan & C, F \\
\hline Areas identified as significant under the RAMSAR Treaty & H \\
\hline Areas supporting rare or unique plant communities & C, H \\
\hline Areas designated as Sole Source Groundwater Aquifers & I, L \\
\hline Areas protected by the Safe Drinking Water Act & I, L \\
\hline City, County, State, and National Parks & D, F, H, L \\
\hline Areas supporting threatened or endangered species & B, C, F, H, I \\
\hline Areas with unique geological features & H \\
\hline Areas protected by the Wild and Scenic Rivers Act & D \\
\hline Areas protected by the Wilderness Act & D \\
\hline
\end{tabular}

1Program Authority / Agency

$A=$ Bureau of Indian Affairs

$B=$ National Marine Fisheries Service

$\mathrm{C}=$ U.S. Fish and Wildlife Service

D = National Park Service

$E=$ State Coastal Zone Office

$\mathrm{F}=$ State Departments of Natural Resources, Fish and Game, etc.

$\mathrm{G}=$ State Historic Preservation Office

$\mathrm{H}=$ State Natural Heritage Offices

I = U.S. Environmental Protection Agency

$\mathrm{J}=$ Federal Emergency Management Agency

$K=$ Natural Resources Conservation Service

$\mathrm{L}=$ Local Government Agencies 


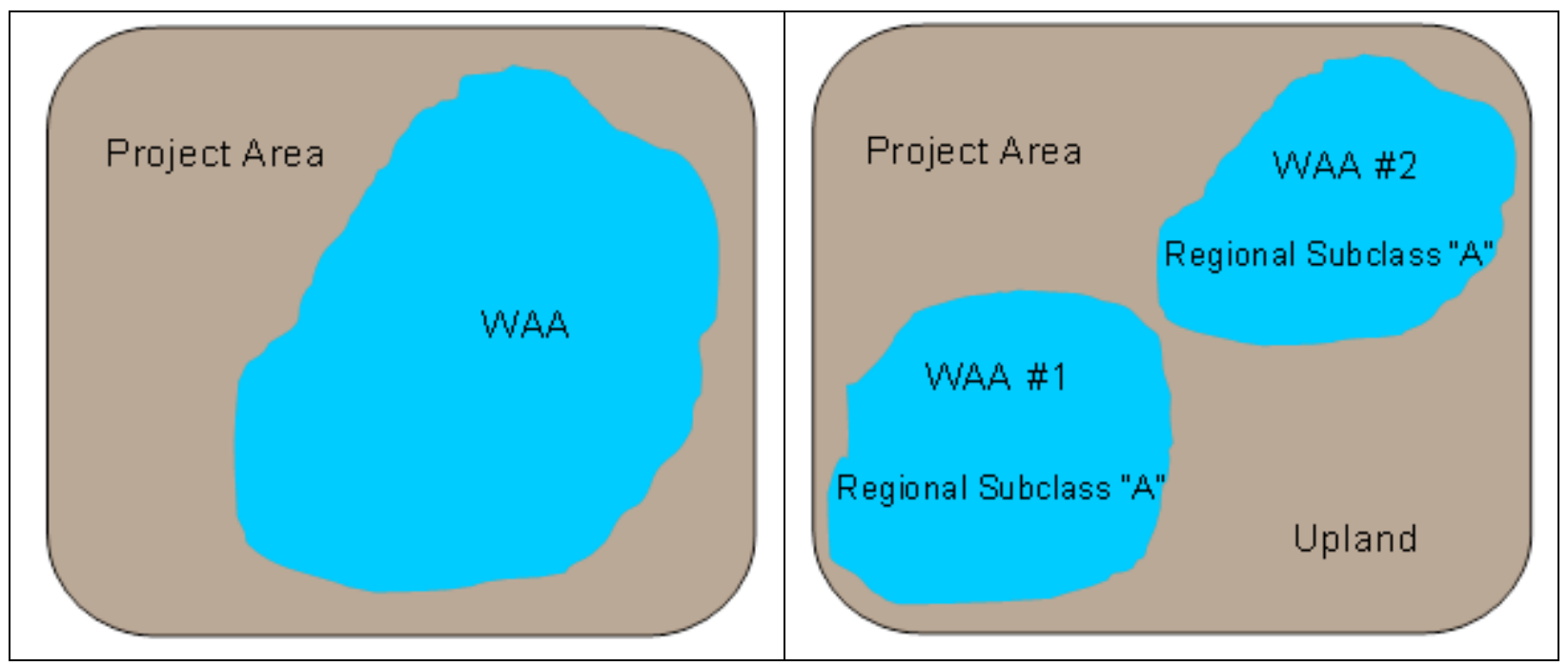

Figure 39. A single WAA within a project.

Figure 40. Spatially separated WAAs from the same regional subclass within a project.

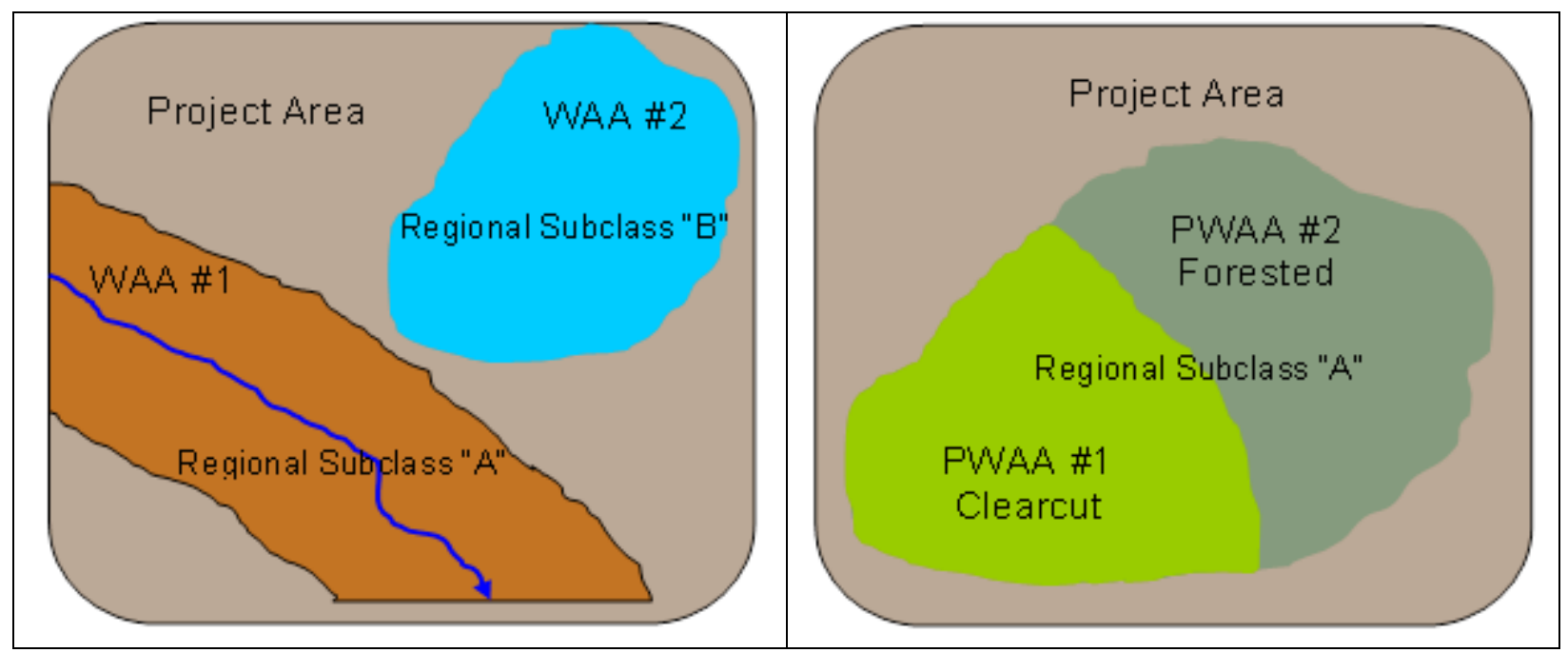

Figure 41. More than one regional subclass within a project area.

Figure 42. PWAAs defined on the basis of differences in site-specific characteristics.

The guidebook should assist the user in evaluating the possible situations that could result in designation of more than one WAA within the project area. At least three situations necessitate defining and assessing multiple WAAs (Smith et al. 1995). The first situation exists when widely separated wetland patches of the same subclass occur in the project area (Figure 40). The second situation exists when more than one wetland subclass occurs within a project area (Figure 41). The third situation exists when a physically contiguous wetland area of the same subclass exhibits spatial heterogeneity with respect to hydrology, vegetation, soils, disturbance history, or other factors that translate into a significantly different value for one or more of the site-specific variable measures. These differences 
may be the result of natural variability (e.g., zonation on large river floodplains) or cultural alteration (e.g., logging, surface mining, hydrologic alterations, Figure 42). Users of the Guidebook should be instructed to designate each of these areas as a separate WAA, with a separate assessment on each area.

There are elements of subjectivity and practicality in determining what constitutes a significant difference in portions of the WAA. Field experience with the wetland subclass under consideration should provide a sense of the range of variability that typically occurs, and the Guidebook should suggest specific criteria for defining multiple WAAs. For example, in Headwater Slope wetlands, recent logging in a portion of a wetland area is a commonly encountered criterion for designating two WAAs (Figure 43).

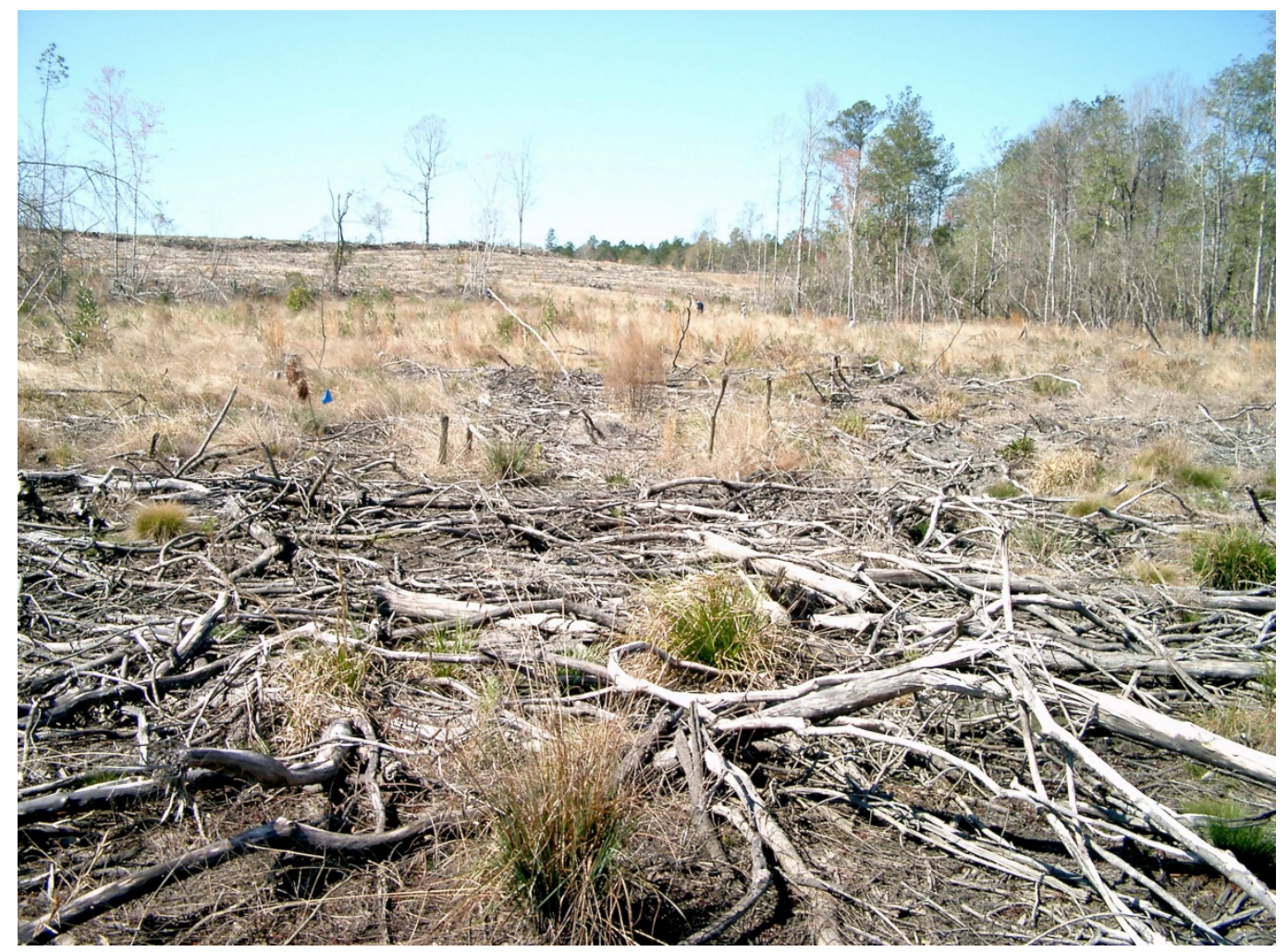

Figure 43. Recent logging in a portion of a headwater slope wetland.

However, the presence of relatively minor differences resulting from natural variability, such as canopy openings due to natural tree fall, should not be used as a basis for dividing a contiguous wetland into multiple WAAs. Distinct zonation caused by various hydrologic regimes or disturbances caused by rare and destructive natural events (e.g., hurricanes) may be appropriate criteria for defining separate WAAs. In general, Guidebook 
users should be encouraged to establish multiple WAAs only where there are clear differences among sites, because data summarization and analysis become increasingly complicated as the project is fragmented into subunits.

\section{Determine the wetland subclass}

HGM assessment requires accurate identification of wetland subclasses to determine if an applicable Guidebook is available. Chapter 3 of each potentially applicable guidebook provides criteria for recognizing subclasses, preferably in the form of a dichotomous key. Current aerial photographs, topographic maps, soils maps, NWI maps, local knowledge, or other available information can be used to help determine which subclasses exist within the project area. In many cases it will not be possible to determine the wetland subclass from remotely sensed data or maps, and an onsite investigation will be necessary.

Figure 44 shows how geomorphic mapping and aerial photography can be used to identify wetland classes for a proposed project area. Decisions made from photo interpretation are normally verified in the field during field reconnaissance.

\section{Collect metric data}

Guidebook users should be provided with very specific and complete directions for collecting and recording data. Collection of field data will require methodology that is specific for the subclass of wetland being evaluated. A checklist is helpful to prepare for the field assessment (Figure 45). It is very important to make sure that the sampling design does not create bias in data collection. The Guidebook should assist the user in determining the minimum number of samples needed for assessment. For example, recommendations can be based on the degree of variability observed during reference data collection, or on some direct measure of variability such as species area curves.

Determining the minimum number of samples needed to characterize a model variable should be based on the size and heterogeneity of the WAA. For example, Klimas et al. (2004) worked with forested wetlands in the Delta Region of Arkansas. They recommended three or four 0.04-ha plots, each containing transects and subplots, for a relatively homogenous WAA that is small, about 1 ha. Figure 46 shows a typical field sampling plot layout that was used for sampling low-gradient riverine wetlands in western 
HGM Classes

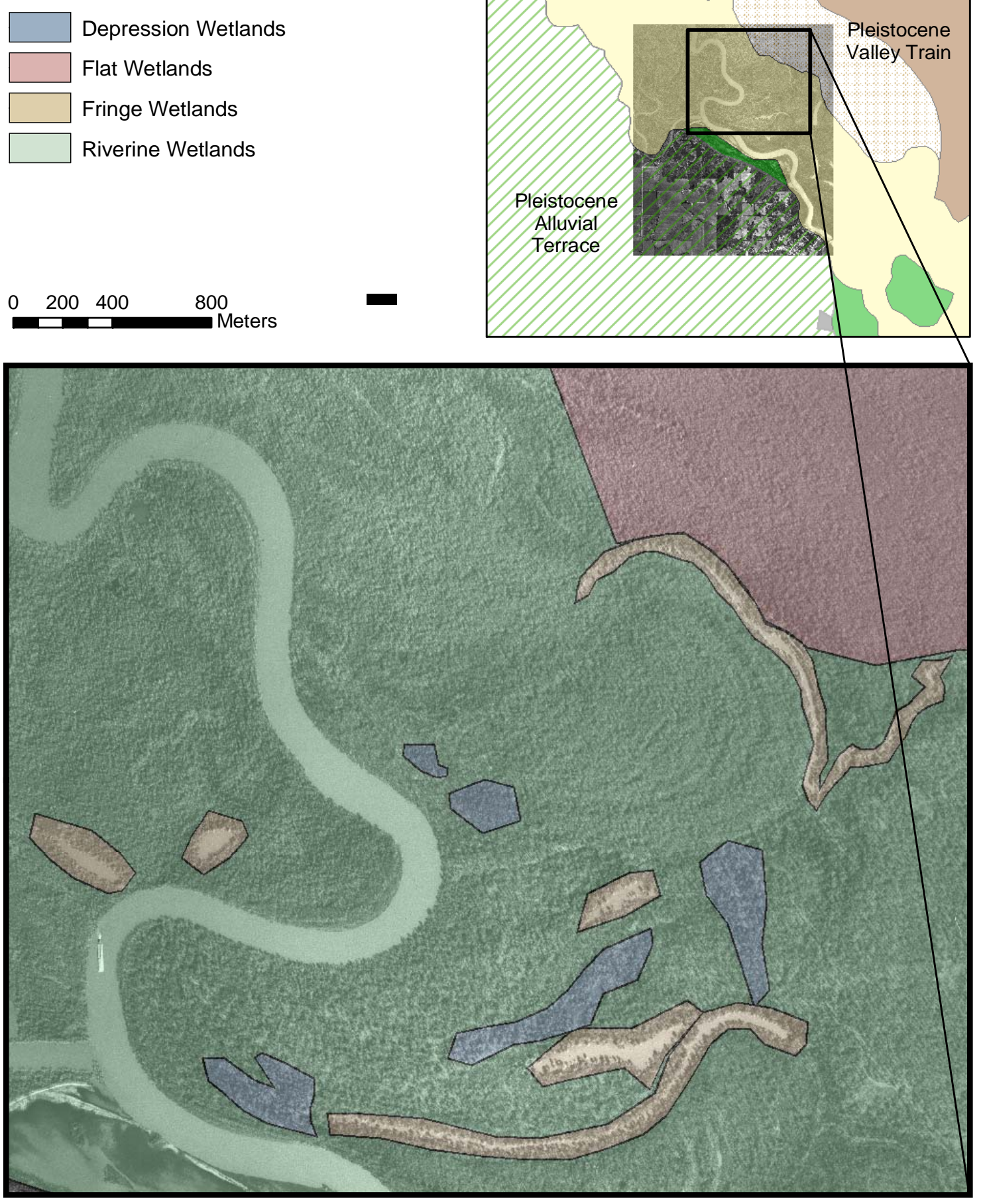

Figure 44. Example application of geomorphic mapping and aerial photography to develop a preliminary wetland classification for a proposed project area. 


\begin{tabular}{|l|l|}
\hline \multicolumn{2}{|l|}{ Field Data Collection Checklist } \\
\hline & copy of assessment protocol \\
\hline & copies of field data sheets \\
\hline & plant identification keys \\
\hline & soil probe or sharpshooter shovel \\
\hline & $50-m$ measuring tape \\
\hline & stakes \\
\hline & flagging \\
\hline & pin flags \\
\hline & large calipers or dbh tape \\
\hline & tally meter or counter \\
\hline & 1-m² frame \\
\hline & GPS receiver \\
\hline
\end{tabular}

Figure 45. Field data collection checklist.

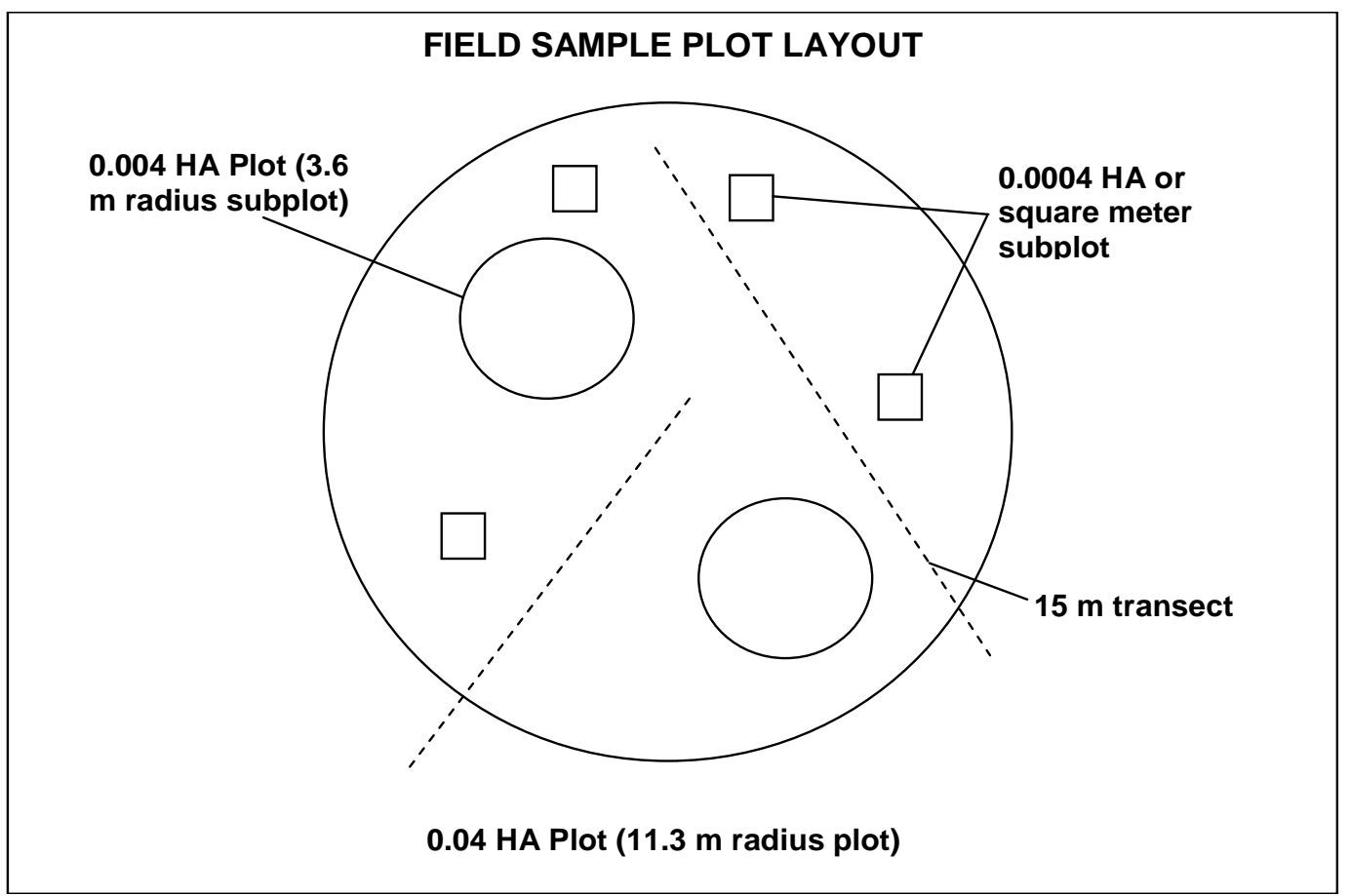

Figure 46. Sample plot layout for riverine wetlands in Western Tennessee.

Tennessee (Wilder and Roberts 2002). In the figure, a 0.04-ha plot is shown that contains two 0.004-ha subplots, four 0.0004-ha (or 1-m²) subplots, and two 15-m transects.

Methods for measuring each variable should be described in sufficient detail in the guidebook so that users can make the required measurements consistently and with precision. Information needed to estimate the variables used in models to assess wetland functions will be collected at 
various spatial scales. For example, landscape-scale variables describe conditions in the wetland's catchment or watershed, and are evaluated using aerial photographs, maps, and field reconnaissance of the area surrounding the WAA. Examples of landscape-scale variables include change in catchment size ( $\left.\mathrm{V}_{\text {CATCH }}\right)$, upland land use (VUPUSE), and habitat

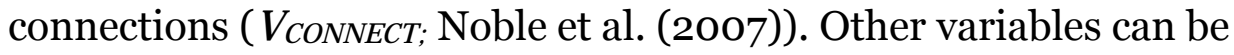
evaluated with a walking reconnaissance of the WAA itself; for example, hydrologic alterations ( $\mathrm{V}_{\text {HYDROALT; }}$ Noble et al. (2007)). Finally, detailed, site-specific data collected within sample plot(s), transects, or subplots at representative locations within the WAA are needed to estimate some variables. For example, canopy tree diameter $\left(\mathrm{V}_{\text {CTD }}\right)$ and canopy tree density ( $\mathrm{V}_{\text {CTDEN; }}$ Noble et al. (2007)).

The following is an example of how field procedures should be presented within a guidebook. This example is for the variable Total Vegetative Cover of Native Emergent Wetland Species ( $\left.V_{\text {COVER }}\right)$ in the Tidal Fringe wetlands subclass of the Gulf Coast Region (from Shafer et al. (2007)).

This variable should be measured during the growing season using the following procedure:

1. Select one or more representative areas within the site for sampling. Beginning at the edge of a shoreline or tidal creek, establish one or more transects perpendicular to the shoreline or along the hydrologic gradient (e.g., increasing elevation). If there are multiple vegetation community types within the WAA, the transect should intersect each vegetation community to ensure a representative sample.

2. Using a standard $1-\mathrm{m}^{2}$ frame, estimate total percentage cover of native nonwoody marsh (OBL or FACW) species using the Braun-Blanquet cover class categories (Mueller-Dombois and Ellenberg 1974). Both live and standing dead emergent plant material should be included. Tidal creeks and other areas where water depths are too deep to support the growth of emergent vegetation should be excluded. The number of transects and plots will depend on the size and heterogeneity of the site; a minimum of 10 plots per transect is recommended for all except the smallest sites.

3. Calculate the total percentage cover of each plot by summing the cover class midpoints for each species, then divide by the number of plots sampled to obtain the mean percentage cover of the study area.

4. Using the table provided, determine the variable subindex that corresponds to the mean percentage cover estimate. 
Sample data sheets should be included in this chapter of the guidebook. Data sheets should include measurement units. They should be organized by the type of measurement; for example, plot size/transect/landscape, etc. They should also provide prompts where needed, for example, the minimum diameter at breast height of trees, depth of soil sample observations, etc. Also, a column is usually included for entering values that will be used in data summarization, for example averages. A sample data sheet from the HGM assessment of Flats Wetlands in the Florida Everglades is shown in Figure 49 (Noble et al. 2002). This data sheet is organized by the scale at which the data are collected. It is important to organize the data so that they can be easily entered into spreadsheets for calculating functional capacity index (FCI) for each WAA.

\section{Analyze the data}

A guidebook will include graphical representations (subindex curves) of the relationships between assessment variables and subindex values, which are constructed as described in Chapter 4 (Smith and Wakeley 2001). Subindex values can be determined by visually comparing the values obtained in the field assessment with the subindex curves, and FCI values are determined by inserting the subindex values into the assessment models. Alternatively, spreadsheets have been developed for several existing HGM models to automatically calculate variable subindex scores and FCI values. Model developers should consider creating a spreadsheet to accompany new assessment protocols.

For assessment areas in which multiple sample plots of equal size were evaluated, the FCI scores calculated for each plot can be averaged in order to generate a single FCI score for each WAA. For example, if a wetland assessment consisted of four sample plots with calculated FCI scores of 0.7 , $0.5,0.8$, and 0.4 , the average FCI score applying to the entire assessment area would be 0.6 .

The third step of data analysis is to calculate the FCUs for each assessed function. This is accomplished by multiplying the FCI by the area of the WAA (Smith et al. 1995). For example, if the FCI for the Detain Floodwater function of a riverine wetland is 1.0 and the area of the WAA is 10 ha, then the FCU for this function is 10. As described above, the manual calculation of subindex and FCI values can be easily automated using simple spreadsheets. Figure 50 is an example of such a spreadsheet, where field data are transferred directly from the data sheets to the spreadsheet input 
form, and FCI values are calculated and multiplied by the area (hectares) of the WAA to generate FCUs (Klimas et al. 2005). For projects involving multiple WAAs, the FCU score can be added in order to generate a single FCU for an entire project area. For example, a large construction project may impact two WAAs. The first assessment area received an FCI score of 0.8 and measured 5 hecares, generating an FCU value of 4 . The second assessment area received an FCI score of 0.5 and measured 10 hectacres, generating an FCU value of 5 . A combined FCU value of 9 should be applied to the area encompassing both WAAs. This final step can also be automated using spreadsheets.

\section{Apply assessment results}

Once the assessment and analysis phases are complete, the results can be used to compare the level(s) of function in the same WAA at different points in time or in different WAAs at the same point in time.

A guidebook should clearly state that at least two assessments will generally be needed to evaluate project-related impacts. The first assesses the number of functional capacity units (FCUs) provided by the site in its preproject or baseline condition. The second assesses the number of FCUs provided by the site in a post-project state, based on proposed project plans and the associated changes to each of the model variables. The difference between preproject and post-project conditions, expressed in numbers of FCUs, represents the potential loss of functional capacity due to project impacts. Conversely, in a mitigation scenario, the difference between the current condition and the future condition of a site, with mitigation actions implemented and successfully completed, represents the potential gain in functional capacity as a result of restoration activities. However, since the mitigation project is unlikely to become fully functional immediately upon completion, a time lag should be incorporated in the analysis to account for the time necessary for the mitigation site to mature and demonstrate improved functional capacity.

Spreadsheets that can be used to help evaluate project impacts and estimate mitigation requirements are available on the internet at: http://el.erdc.usace.army.mil/wetlands/datanal.html. A guidebook should include a specific discussion of how to estimate changes in variable values resulting from impacts or mitigation actions relative to the particular regional wetland subclass(es) covered by the document. 


\section{Validate Assessment Models}

\section{What is validation?}

All models are an approximation of reality and consequently can never fully describe the real system. Nonetheless, models can be useful because they can help to understand complex systems and predict the effects of environmental change (Hall and Day 1977). In the HGM Approach, the validation of assessment models involves comparing the functional capacity index (FCI), derived from an assessment model, to an independent, quantitative measure of the function.

In some ways the validation of an assessment model is similar to a hypothesis test in statistics, where the results of a test determine whether a hypothesis is accepted or rejected at a specified level of confidence (Caswell 1976, Overton 1977, Marcot 1983). Assessment models represent a hypothesis that is being tested by comparing the FCI derived from an assessment model to an independent, quantitative measure of function. In this case, however, the objective is not to accept or reject the assessment model in a statistical sense. Rather the objective is to determine if the accuracy and precision of the assessment model is acceptable for assessing wetland functions. The success criteria for validating a wetland assessment is determined by model developers, end users, the assessment team, or by those conducting the validation effort.

\section{Why validate?}

The validation of assessment models ensures that changes in wetland function are reflected accurately in the direction and magnitude of change in the FCI. This in turn ensures that wetland impacts and mitigation credits are estimated in a comparable and consistent manner, and that there will be no unintended gain or loss in wetland function due to a project. Validation has additional practical advantages to both developers and end users of assessment models, including the ability to:

1. Strengthen the technical credibility and defensibility of the HGM Approach.

2. Reduce subjectivity and ambiguity in the definition of wetland functions.

3. Reduce individual bias in model development and application. 
4. Provide an objective basis for choosing between alternative models.

5. Reduce arguments and litigation over the technical aspects of assessment models.

\section{Who validates?}

It seems logical that the developers of a guidebook have the most interest in pursuing model validation. However, the time and effort required to validate assessment models are often beyond the mandate and resources of most, largely voluntary assessment teams. End-users (e.g., regulators, consultants, managers) are also logical candidates for performing validation work. Third parties (e.g., university researchers and their graduate students) are also potential candidates for validation work.

\section{Approaches to validate assessment models}

There are two basic approaches to the validation of assessment models. The first involves experimental manipulation of specific attributes or processes at one or more reference wetlands to see whether the assessment model is able to predict the changes in functional capacity that are observed at the site following manipulation. For example, an assessment model for the Particulate Retention function may predict that the sediment-trapping capacity of a floodplain wetland will be reduced by $30 \%$ if all large trees are removed. A test of the model might consist of measuring sediment accretion for a period of time under existing conditions, then harvesting all large trees and measuring the change in accretion rates. This approach may provide the truest test of model performance (Schamberger and O'Neil 1986), given that the primary use of assessment models is to predict changes in wetland function due to project-related disturbances. However, experimental manipulation is rarely undertaken because of the difficulty in finding areas that can be manipulated, and because of the long period of time that may be required for the results of the manipulation to be manifested. An alternative to prescribed experimental manipulation is to monitor changes at locations that are currently being altered, or were previously altered, due to either project impacts or restoration activities.

The second approach to model validation is to compare the FCI derived from an assessment model to an independent, quantitative measure of function. This approach to validation does not involve experimental manipulation, but it normally requires a significant investment of time and resources to collect the necessary data. The actual effort required will 
depend on many factors including the number of sample locations, distance between sample locations, difficulty of collecting information on the independent measure of function, and the length of time required to acquire information and data that are representative of conditions in reference wetland sites. The following section focuses on the application of independent measures to assessment model validation because it is the more practical.

In conceptualizing assessment models, it is the responsibility of the assessment team to identify potential independent, quantitative measures of function for each function ascribed to a wetland subclass (Section in Chapter 3 titled "Define Wetland Functions"). For example, the amount of sediment accretion in a specified area over a specified period of time could be used as an independent measure of function for a model that assesses the capacity of a wetland to retain sediments (Kleiss 1996). Similarly, the volume of floodwater retained per unit area over a specified time period is a potential independent measure for the surface water storage function, the number of kilograms of nitrate transformed per unit area per year is a potential independent measure for the nutrient transformation function, and the total number of breeding vertebrate species identified in reference wetlands over a specific period is a potential independent measure for the maintenance of a characteristic wildlife community function. Published guidebooks provide many more examples of independent measures of function (http://el.erdc.usace.army.mil/wetlands/hgmhp.html).

Examples of validating assessment models developed using the HGM Approach include Pohll et al. (1999), Carroll et al. (2000), and Hill et al. (2006). Many more examples of validating model output using an independent standard of comparison have been completed in the context of Habitat Suitability Index (HSI) models developed for the Habitat Evaluation Procedures (HEP) (U.S. Fish and Wildlife Service 1980, 1981). Some examples relevant to the HGM Approach include Lancia et al. (1982), Cook and Irwin (1985), O’Neil et al. (1988), O’Neil (1993), and Adamus (1995).

\section{Relationship between the $\mathrm{FCl}$ and independent measure of function}

In the HGM Approach, the FCI is an index expressed on a ratio scale ranging from $o$ to 1 . The ratio scale of measurement has two important features (Zar 1999). The first is that the magnitude of change between similar intervals is the same. For example, the magnitude of change in FCI 
from 0.2 to 0.3 is the same as the magnitude of change in FCI from 0.8 to 0.9. The second is that the ratio scale includes a zero point, and that the zero point has a physical significance. In the HGM Approach, an FCI of zero indicates the condition in which a wetland has no capacity to perform a function.

Independent, quantitative measures of wetland function are also characterized by ratio scales. Examples include counts of items, lengths, weights, volumes, rates, and units of time. In the HGM Approach, it is assumed that there is a one-to-one relationship between FCI and the capacity of the wetland to perform a function. Consequently, the expected relationship between FCI and independent measures of function is linear (Figure 47, Graph A) (Ott 1978).

The functional capacity that corresponds to an FCI of 1.0 (denoted as $\mathrm{X}_{\mathrm{RS}}$ in Figure 47, Graph A) varies with the wetland subclass and other factors. The numerical value, or range, of $\mathrm{X}_{\mathrm{RS}}$ is determined by the functional capacity exhibited in reference standard wetlands and is estimated during model calibration (see Chapter 6, "Develop End-User Assessment Protocol”). Therefore, $\mathrm{X}_{\mathrm{RS}}$ is normally not a single value, but rather represents a range of metric values corresponding to the range of functional capacity exhibited by reference standard wetlands. For example, if reference standard wetlands export organic carbon at rates ranging from 21 to $35 \mathrm{~kg} / \mathrm{ha} /$ year, this constitutes the range of values assigned an FCI $=1.0$ (Figure 47, Graph B).

For some functions, there are no disadvantages to even higher levels of function and, therefore, no decline in FCI. Say, for example, that the number of breeding forest-interior bird species at reference standard sites ranged from 13 to 16 ; this range would represent $\mathrm{X}_{\mathrm{RS}}$ in an assessment model designed to predict species richness of forest-interior birds. However, another site not considered to be a reference standard may contain 18 species. For this function, the assessment team would probably design the model to give sites having unusually high levels of function (i.e., bird richness) an FCI of 1.0 (e.g., line a in Figure 47, Graph B). On the other hand, when certain factors occur at unnaturally high levels it can lead to a reduction in the capacity of a wetland to perform other functions. Brinson (1995) used the example of increased rate of sediment transport into a wetland due to clearing of surrounding upland forests. Levels of sediment input and retention that exceed those exhibited by reference standard 

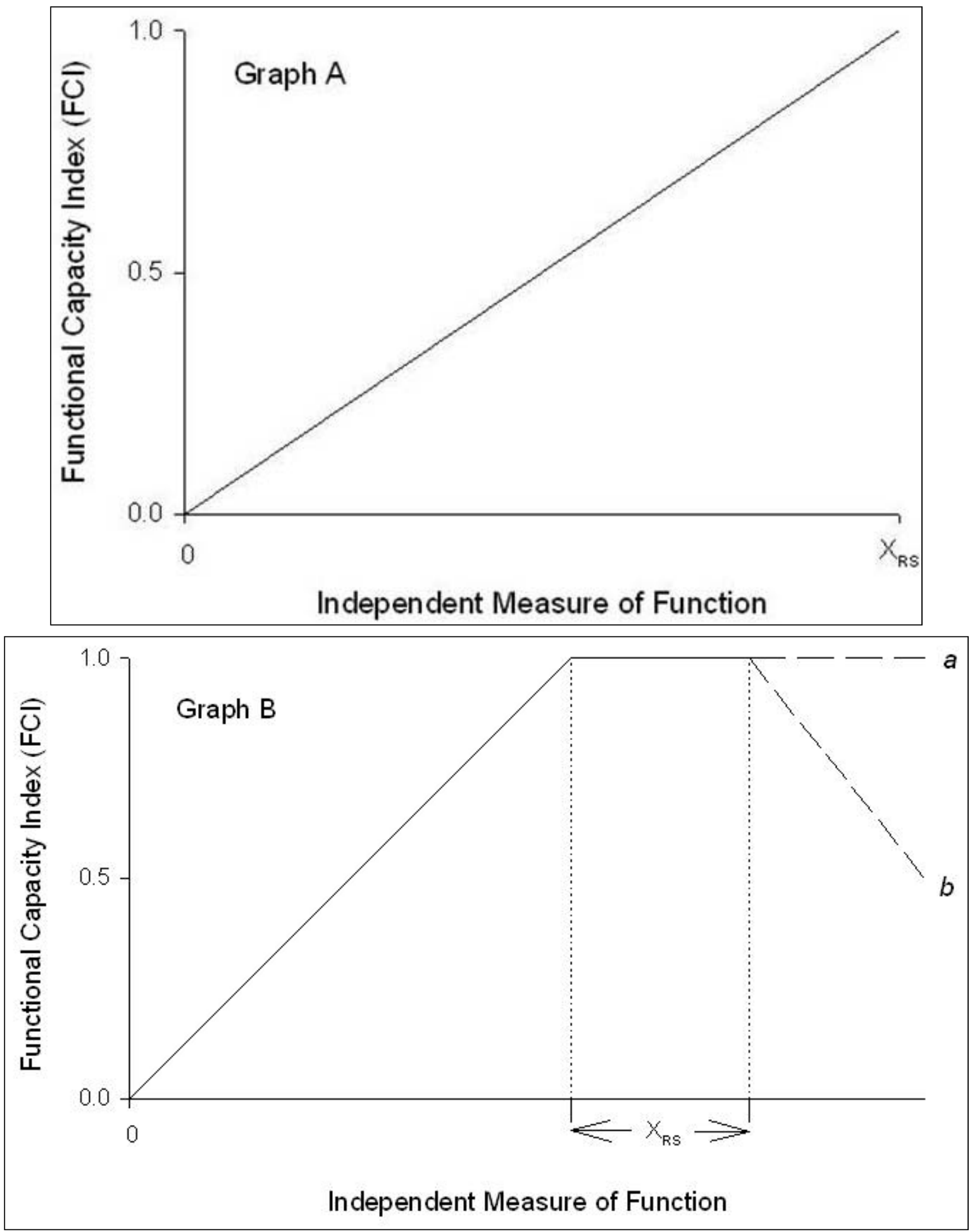

Figure 47. Expected relationship between the modeled Functional Capacity Index ( $\mathrm{FCl}$ ) and the independent measurement of function.

wetlands should be reflected in a decline in FCI (e.g., line b in Figure 47, Graph B). Recognize that it may be difficult to determine the slope of this line without considerable research and fieldwork. 
The basic design of a model validation study is to identify a series of wetland sites, apply the model to each site to estimate FCIs for a particular function, independently measure the magnitude of function, compare FCI and the independent measure of function across all sites and, if needed, modify the draft model to bring FCI scores and independent measures into better agreement. All direct measures incorporate some level of measurement error that causes "scatter" in the data set. There is little one can do about measurement bias, except to select the most reliable techniques by reviewing published literature and talking with experienced individuals. Measurement error depends on the sampling design and sample sizes used to estimate the magnitude of function; the amount of error can be quantified statistically (e.g., standard error of the mean, confidence limits). It is important to remember that both measurement error and bias in the independent measure of function can reduce the strength of the relationship with FCI.

\section{Testing the assessment model versus assessment model components}

Assessment models have several components that are amenable to validation (Schamberger and O’Neil 1986). Many assessment models utilize metrics that are easily measured surrogates or proxies of the actual quantity of interest (Smith et al. 1995). For example, the frequency of overbank flooding variable could be estimated using metrics such as presence of wrack lines, silt deposits, or characteristics of the vegetation (e.g., proportion of the dominant plant species in the community that are wetland species). These metrics can be validated by measuring them in areas of known flooding frequency. Similarly, an assessment model for Organic Carbon Export from forested riverine wetlands may use Tree Canopy Cover as a variable based on the assumption that canopy cover is directly related to the abundance of organic debris available for export. Validation of this assumption might involve comparisons of canopy cover measurements against the mass of leaves and twigs collected in litter traps within a number of floodplain wetlands. Testing the validity of specific metrics can be critical to the quality of assessment models because many important variables (e.g., hydrologic and biogeochemical variables) are difficult or impractical to measure directly. The use of indicator variables in models introduces additional variability that can weaken the relationship between model output and actual measurements of wetland function. Careful metric selection and validation can reduce unwanted variability and improve model accuracy. 
A second level of validation involves testing the relationship between the metric values and the subindex. Like the FCI, subindices are an estimate of functional capacity that by definition range from 0 to 1 . The relationship between the metric value and the subindex can be tested by plotting subindices against the independent measure for the function. This relationship should approximate the FCI curve or histogram for the assessment model, except for the effects of other variables in the model.

Finally, validation can target the whole assessment model. For example, one can test the accuracy of an assessment model for the Wildlife Community Support function by first calculating the FCI for each wetland, and then independently measuring the function specified for each wetland. For example, if the independent measure of function is the combined species richness of breeding terrestrial vertebrates, this is measured at each site using appropriate sampling techniques for each component of the vertebrate community (i.e., birds, mammals, reptiles, and amphibians). The combined number of species of vertebrates at each site is calculated, plotted against FCI. This provides a basis for interpreting the accuracy and appropriateness of the functional model (Figure 48).

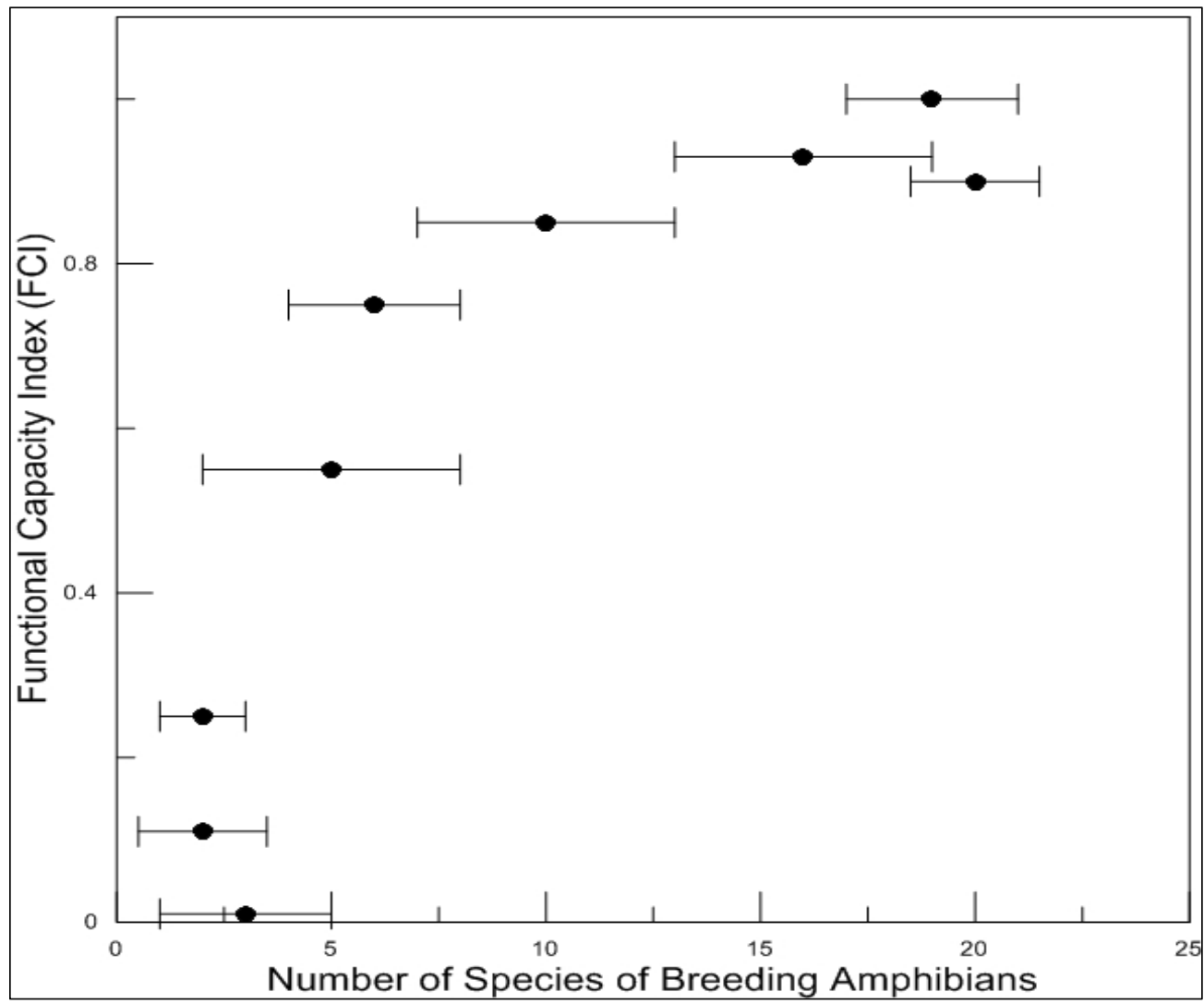

Figure 48. Relationship between the $\mathrm{FCl}$ and an independent measure of function (i.e., number of species of breeding amphibians captured in each wetland). 
In practice, it is probably most efficient to first test the assessment model and then, if needed, examine one or more of the individual metrics or the underlying assumptions of the assessment model. An assessment model that passes the first test may not need to be tested further for users to have confidence in its predictions. This is no guarantee, however, that all components of the model are necessary or are performing properly. Furthermore, if the overall model does not meet performance expectations (as defined by the assessment team or end user), it will be necessary to test each of its parts. Model validation is an iterative process involving testing, modifying, and retesting until standards for reliability are achieved.

\section{A generic procedure for validating assessment models}

The following procedure for validating assessment models is based on correlations between the FCI derived from an assessment model and the independent measure of function. It is similar to the method described by O’Neil et al. (1988) for testing and modifying Habitat Suitability Index models. The procedure is outlined in Table 21.

Table 21. Generic procedure for validating assessment models.

1. Select at least 10 to 20 or more reference wetland sites that represent the full range of conditions in the reference domain.

2. Collect data for all metrics in the assessment model, and for any metrics that may be considered under alternative versions of the assessment model, at each reference wetland site. Calculate an $\mathrm{FCl}$ for each reference wetland site.

3. Collect data for all independent, quantitative measures of function and variables at each reference wetland site. Use an accepted sampling method and a design that minimizes bias and measurement error. It may be necessary to collect data over a period of one, or more, wet-dry cycles in order to determine average conditions.

4. Based on the data collected for the independent measure of function and variables, re-evaluate assumptions made during the development of the conceptual assessment model and testing and calibration of variable metrics in terms of the relationship between the range of variable metric values in reference standard wetlands and the level of functional capacity that corresponds with $\mathrm{FCl}=1.0$.

5. Examine plots and coefficients of determination $\left(r^{2}\right)$ of $\mathrm{FCl}$ versus the independent measure of function. The expected relationship is linear, as in Figure 50, at least for the ascending or descending initial limb of the graph.

6. If needed, examine plots of the relationships between the measure (x-axis) for each variable in the model and the independent measure of function (y-axis). The plots should resemble the curves or histograms given in the functional model, except for the effects of other variables on model output.

7. If needed, modify metric/subindex relationships, add or drop variables, or adjust the mathematical equation used to derive the $\mathrm{FCl}$, to improve the correlation between $\mathrm{FCl}$ and the independent measure of function. Also, test and compare alternative versions of the assessment model.

The first step is to select reference wetlands that represent the range of conditions exhibited by the wetland subclass in the reference domain. At 
least 10 to 20 sites are recommended. Include two or three sites that represent reference standard conditions (i.e., $\mathrm{FCI}=1.0$ ). The remaining sites should represent the range of disturbance conditions that occur in the reference domain. It is permissible to use some of the model calibration sites, if they meet the guidelines above, provided the calibration step did not already include consideration of actual measures of function at those sites.

Next, apply the assessment model at each validation study wetland and calculate FCI values (Table 21, Step 2). At the same time, variables needed for any potential alternative versions of the assessment model should also be collected. The purpose of validation is to improve the accuracy of the assessment model by revising the assessment model or replacing it with a more accurate assessment model. In anticipation of potential assessment model revisions, investigators should have alternative versions of the model in mind when designing the validation study, and collect metric values for any variables that may be used to revise the assessment model.

The next step is to measure functional capacity at each selected site, through available literature, or based on input from experts in the field using the independent measure of function identified in the guidebook, (Table 21, Step 3). The length of time necessary to capture the typical levels of function can vary from a few months to several years. The FCI that is derived using an assessment model is meant to indicate the normal or average capacity of a wetland to perform a function. The FCI for an undisturbed wetland should not vary appreciably from year to year, unless succession, or another temporal factor, can influence functional capacity. However, many independent measures of function (e.g., tons of sediment trapped, cubic meters of surface water retained, kilograms of carbon exported, numbers of breeding vertebrate species detected) vary annually and, consequently, more than one year may be needed to determine average conditions.

As mentioned earlier in the section on the expected relationship between FCI and the independent measure of function, investigators should select measurement techniques that are known to be unbiased and use sampling designs that minimize sampling error. This is because any variation in the independent measure of function will affect the strength of the relationship between that measure and FCI. Appropriate measurement techniques can be identified from the literature or by consulting experts. Often specialized equipment or skills are needed, requiring trained and experienced personnel. In addition, the sampling design (e.g., sample size, replication, 
stratifications) and statistical treatment of the data should be carefully planned to minimize error and keep the precision of the measurements within acceptable limits. A measure of precision (e.g., standard error or confidence limits) should accompany each estimate of the independent measure of function.

After values of both FCI and the independent measure of function have been obtained for each selected site, the first step in data analysis is to reevaluate assumptions made during model development and calibration about the level of function in reference standard wetlands (Table 21, Step 4). Reference standard wetlands are selected based not on one function, but on the suite of functions performed by minimally disturbed wetlands in the reference domain (Smith et al. 1995). Now is the time to consider, based on actual measurements of one or more functions of interest, whether the reference wetlands initially selected as reference standard wetlands actually achieve that status. The decision is necessarily subjective, but might be based on the measured level of function at a designated reference standard site in relation to the assessment team's a priori opinion of that site. For example, if the assessment team's concept of a reference standard was initially thought to include sites with capacities for carbon export greater than $90 \mathrm{~kg} / \mathrm{ha} / \mathrm{yr}$, then a site with carbon export of $75 \mathrm{~kg} / \mathrm{ha} / \mathrm{yr}$ may not be an appropriate reference standard site and could be dropped from that status. On the other hand, if other considerations lead to the retention of the site among the reference standards, then the implied range of function for reference standard sites ( $\mathrm{X}_{\mathrm{RS}}$, Figure 5O, Graph B) must be modified to include sites that export $75 \mathrm{~kg} / \mathrm{ha} / \mathrm{yr}$. In any case, the draft model's assumed value of $\mathrm{X}_{\mathrm{RS}}$ should be reevaluated and modified, if necessary, based on the independent measures of function at these sites.

The next step in the validation study is to compare the FCI values derived from the assessment model against the independent measure of function for each wetland site (Table 21, Step 5). The relationship is through the initial ascending or descending limb, and is expected to remain linear until the independent measure of function equals reference standard $\left(\mathrm{X}_{\mathrm{RS}}\right)$ (Figure 50). Therefore, the strength of the relationship can be evaluated with a linear (Pearson) correlation coefficient ( $r$ ) and coefficient of determination $\left(\mathrm{r}^{2}\right)$. The coefficient of determination is an estimate of the proportion of variability in FCI that is due to its relationship with the independent measure of function (Zar 1999). 
Validation should focus mainly on the initial ascending or descending limb of the relationship between FCI and the independent measure of function (Figure 50). This is because the slope and subsequent limbs (e.g., line b in Figure 50 Graph B), if any, are mainly based on professional judgment of the assessment team, rather than any underlying quantifiable relationship between FCI and the measure of function. In addition, during model validation, FCI values from wetland sites that have measured levels of function within the optimal range ( $\mathrm{X}_{\mathrm{RS}}$ in Figure 50, Graph B) should be consolidated and plotted in relation to the lowest value in the $\mathrm{X}_{\mathrm{RS}}$ range. This procedure eliminates the plateau in the curve, resulting in an expected relationship similar to Figure 50, Graph A and, thus, makes the relationship more amenable to testing with linear correlation. This procedure is described below and depicted in Figure 51.

Figure $51 \mathrm{~A}$ is a plot of FCI and an independent measure for a Wildlife Habitat Support function that was intended to reflect the number of species of breeding amphibians present in a wetland. The independent measure was made by counting amphibian species captured during 10 days of trapping in spring using five clusters of pitfall traps (e.g., Block et al. 1994) in each of 10 different wetlands. The figure demonstrates that reference standard conditions are met when the number of amphibian species is equal to or greater than $9\left(\mathrm{X}_{\mathrm{RS}}\right)$. The linear regression line shown in the plot (Figure 51A), demonstrates that a weak linear relationship exists between the number of amphibian species observed and the calculated FCI scores.

To determine whether the draft model is an accurate predictor of amphibian species richness, one must consider (1) the coefficient of determination between FCI and the independent measure of function, and (2) the distribution of plotted points in relation to the expected trend. In the example, the coefficient of determination indicates that about $61 \%$ of the variation in FCI can be accounted for by differences in amphibian species richness. In general, coefficients of determination in excess of 50\% are desirable, as they indicate that the model is able to account for most of the variance in the two sets of measurements. However, a simple Pearson correlation does not take into account changes in slope or the logical y-intercept of the relationship. As a result, correlation and regression analysis should consider the shape of the curve in addition to coefficient of determination values.

There are two ways to modify a draft model to improve its performance relative to the independent measure of function: (1) modify the assessment 
model equation by changing mathematical functions (e.g., arithmetic means versus geometric means), changing weights or exponents, or by dropping or adding variables, or (2) modify the relationships between the measures of one or more metrics and their subindices. Both approaches may be needed to achieve a good fit and both involve some trial-and-error experimentation. The modification of assessment model arithmetic and interpretation of results at the FCI functional scale should be examined as a first step, which is discussed in detail below. However, in some cases, examination of the usefulness and scaling of individual model variables may be required in cases where the relationship's functional values and independent validation measures remain unclear.

For example, the data shown in Figure 51 are for a four-variable model of the general form $\mathrm{FCI}=\left(\left(\left(\mathrm{V}_{\mathrm{A}}+\mathrm{V}_{\mathrm{B}}\right) / 2+\left(\mathrm{V}_{\mathrm{C}}+\mathrm{V}_{\mathrm{D}}\right) / 2\right) / 2\right)^{1 / 2}$. One way to improve the correspondence between the data points and the expected trend is to drop the exponent on the aggregation equation, which is equivalent to squaring the right side of the equation. Squaring values of the index does not affect the end points appreciably, since $0^{2}=0$ and $1.0^{2}=1$. However, squaring lowers values in the midrange of the index (e.g., $0.5^{2}=$ 0.25). Therefore, squaring reduces the curvature of the data plot shown in Figure 51 and helps to bring FCI values into line as seen in Figure $51 \mathrm{C}$. This modification improves the correlation of FCI versus the independent measure of function to $r=0.88$ and $r^{2}=0.78$. Regression analysis of the ascending limb portion of the curve shows that the fit of the data to the expected trend is now $\mathrm{r}^{2}=0.94$.

In some cases, it may be appropriate to force the y-intercept through zero as shown in Figure 51D. This should be considered when the independent measure selected represents an essential requirement of wetland functionality. For example, if a function is designed to detain floodwater, some frequency of flooding is required to provide the function, and complete lack of flooding prevents any floodwaters from contacting wetlands where detention might occur. Figure 51D expresses the relationship between amphibians and FCI scores for a model specifically designed to measure habitat for amphibians, thus justifying the forcing through zero. However, in the case of many hydrology, habitat, and biogeochemical functions the selection of independent measures capable of determining the zero level of wetland function is more challenging. Since most assessment models bundle functions into groups (e.g., examine habitat at the floral or faunal scale), a lack of a single component may not preclude functioning from other sources. For example, in a general habitat assessment model designed to capture functional support for birds, vegetation, or other organisms, the 
lack of one species group selected as an independent validation measure (e.g., amphibians) may not indicate a total loss of function. As a result the yintercept should only be forced through zero in cases where the absence of a given independent validation measure precludes the function of interest.

One type of model modification that should always be considered is dropping one or more variables, particularly if the model contains a total of more than four or five variables. Model simplification by dropping unnecessary or unimportant variables has the added benefit of reducing the amount of time and effort required for users to apply the model and to gather data in the field. Approaches to identify variables that might be dropped without reducing model performance appreciably are discussed in the section in Chapter 5 titled "Test Suitability of Variables and Metrics." Another approach is to examine the relationship between the measure of each variable and its subindex by plotting the average measure for a variable at each site against the independent measure of function (Table 21, Step 6).

If a variable is important to the performance of the model, then a plot of the variable metric versus the independent measure of function should resemble the relationship between the metric and its subindex. Deviations or outliers should be explainable in terms of the influence of the other variables in the model. For example, Figure 49 shows a hypothetical relationship between total organic carbon export (i.e., the independent measure of function) measured in a series of 20 low-gradient riverine wetlands and the percent cover of leaves and fine woody debris determined in sample plots within those wetlands (i.e., variable $\mathrm{V}_{\text {LITER }}$ of Ainslie et al. $(1999))$. FCI $=1.0$ corresponds to a carbon export rate of approximately $95 \mathrm{~kg} / \mathrm{ha} / \mathrm{yr}$, the lowest value measured at reference standard sites.

Considerable scatter is expected in the data (Figure 49) because the plot fails to consider the effects of other variables that influence carbon export. For example, the points labeled "A" in Figure 49 are much higher than expected based on the variable/subindex relationship presented in the model. However, these values might have come from sites where larger woody debris contributes more heavily to organic export. Similarly, the points labeled "B" may be from sites that rarely flood, so that accumulated leaf litter does not contribute greatly to carbon export. The full model contains a variable that accounts for coarse woody debris $\left(\mathrm{V}_{\mathrm{CWD}}\right)$ and another describing flood frequency $\left(\mathrm{V}_{\mathrm{FREQ}}\right)$. Therefore, the outlying values of carbon export ("A" and "B" in Figure 49) can be explained based on other variables in the model. 


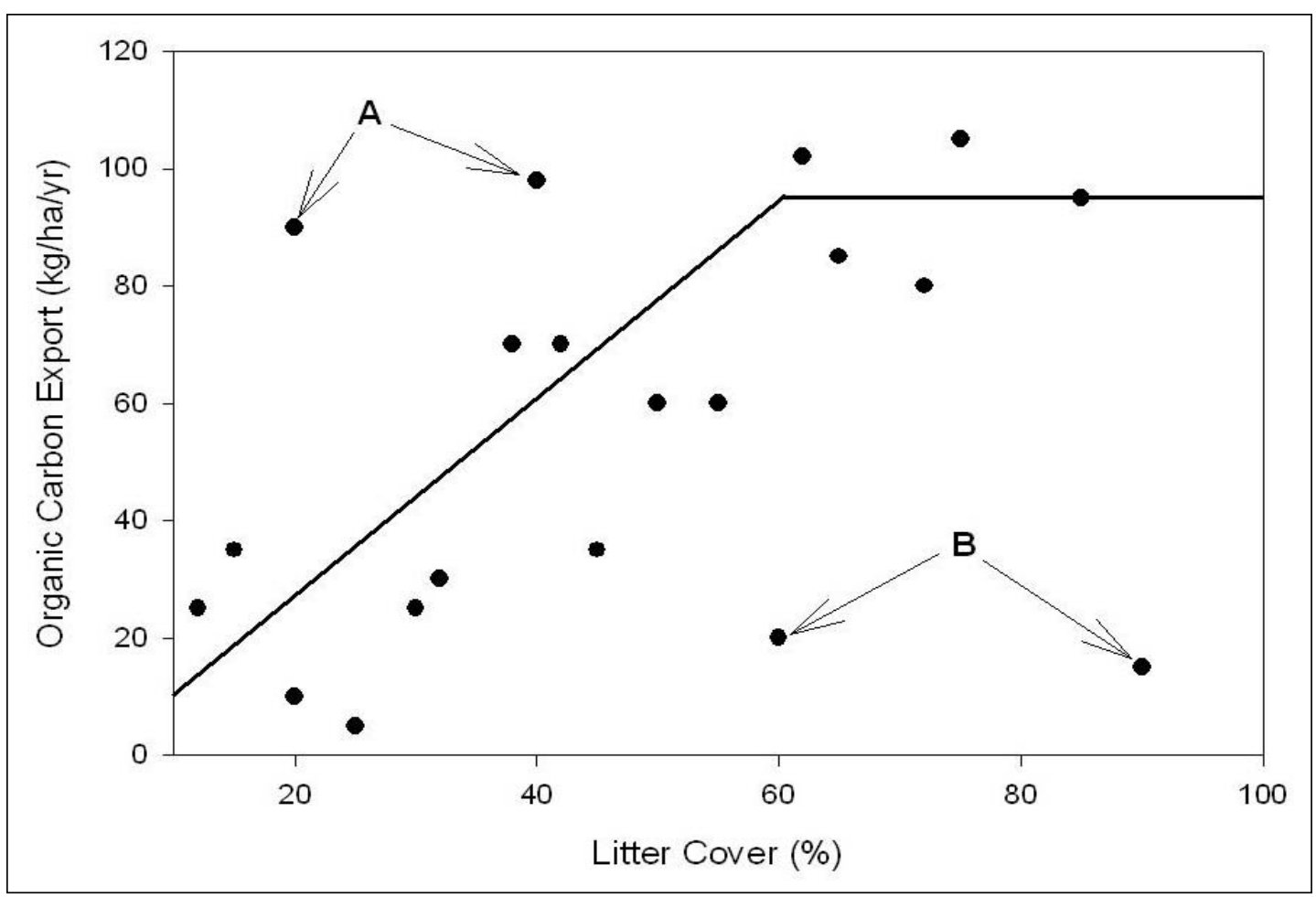

Figure 49. Hypothetical relationship between percent leaf litter cover (i.e., metric) and an average value of total carbon exported from a number of riverine wetlands (i.e., the independent measure of function where the solid line represents the expected relationship based on the assessment model.

Figure 49 suggests that the variable $\mathrm{V}_{\text {LITTER }}$ is indeed an important factor in carbon export, validating the opinion of the assessment team. Except for deviations that can be explained based on the influence of other variables, the increasing trend in the plot is clear. If the graph were to show a random scatter of points, or some trend opposite to the expected one, it would indicate that (1) the variable may be less important than other variables and might be down-weighted or dropped from the model, (2) the variable may be poorly defined or difficult to measure and should be revised or dropped, or (3) the variable may affect carbon export in some way that the assessment team did not anticipate. In the latter case, the relationship between the variable and its subindex could be redrawn to improve model performance.

After all options for revising the assessment model and the relationships between variables have been exhausted, and the effects have been examined by recalculating the overall fit of each new version of the model to the independent measure of function, the result is a revised assessment model that is more accurate and reliable than the draft model was. However, the new model should undergo testing to ensure that any modifications to sample variables and assessment models achieved the desired outcomes. 


\section{Applications for the Assessment Procedures}

\section{Introduction}

Application of individual HGM Guidebooks and interpretation of assessment results to determine mitigation ratios has intentionally been at the discretion of each Corps District. This chapter provides guidelines and examples for applying the procedures in the Guidebook to assess wetland functions or baseline conditions, compare multiple wetlands of the same subclass, compute potential project impacts, determine mitigation requirements, and monitor mitigation success. The Functional Capacity Index (FCI) can be applied to each of these purposes.

\section{Background}

Corps of Engineers regulations require that applications for wetland permits undergo a Public Interest Review that must consider what effects the project will have on "functions important to the public interest." Important wetland functions include hydrologic functions, such as surface water storage; biogeochemical functions, such as cycle organic carbon; and biological functions, such as providing habitat for aquatic and terrestrial flora and fauna.

The HGM Approach is implemented at the local or regional level through the development of Regional Guidebooks that contain models to assess the functions of a particular wetland type in a defined geographic area. The output of each model is an index on a scale of o to 1 that reflects the actual magnitude of function in the target wetland relative to reference wetlands of the same type in the region. Thus, the FCI for each function is readily used to evaluate impacts, compare project alternatives, and help design and evaluate mitigation plans.

\section{Project objectives}

How an HGM Guidebook is applied is dependent on the project objectives. The following examples and discussion illustrate the most common applications of HGM and interpretation of the data from HGM FCI results. However, the HGM approach is not limited to these specific examples. 


\section{Baseline condition}

For many projects, the calculation of existing or baseline FCI values may be all the information that is needed to assess the current functional condition of a wetland compared to the reference standard condition. Baseline values indicate how well the target wetland functions, regardless of scale, relative to the most fully functioning examples of that wetland type in the region considered by the guidebook.

Determining baseline conditions for a wetland represents the comparison of FCIs at a point in time to the Reference Standard Condition. Table 22 illustrates the results of a baseline assessment of a headwater slope wetland (Noble et al. 2007). This type of assessment highlights the differences among functions within a wetland, and can be used to identify the most impacted functions and focus restoration actions on them.

Table 22. Comparison of baseline wetland FCl to Reference $\mathrm{FCl}$ scores.

\begin{tabular}{|l|l|l|l|}
\hline Function & $\begin{array}{l}\mathrm{FCl}-\text { reference } \\
\text { standard }\end{array}$ & $\begin{array}{l}\mathrm{FCl} \text { - sample } \\
\text { site }\end{array}$ & $\begin{array}{l}\text { Difference in } \\
\mathrm{FCl} \text { score }\end{array}$ \\
\hline Water Storage & 1.0 & 0.78 & 0.22 \\
\hline Cycle Organic Carbon & 1.0 & 0.84 & 0.16 \\
\hline $\begin{array}{l}\text { Maintain a Characteristic Plant } \\
\text { Community }\end{array}$ & 1.0 & 0.69 & 0.31 \\
\hline $\begin{array}{l}\text { Provide Characteristic Wildlife } \\
\text { Habitat }\end{array}$ & 1.0 & 0.69 & 0.31 \\
\hline
\end{tabular}

\section{Comparing multiple wetlands of the same subclass}

Comparing FCIs for multiple wetlands within the same subclass follows the same basic procedure as determining the baseline condition (Table 23). This type of comparison may be useful for avoiding impacts to the least disturbed of a group of wetlands in a possible project impact area. It may also be useful in selecting wetlands that have the greatest potential for an increase in functional capacity for restoration. The greatest power of any HGM assessment is to compare the differences between functions.

\section{Compute potential project impacts}

If the objective of the analysis is to compare different project configurations or alternative designs to avoid and minimize impacts to wetlands, then the acreage of wetland impact should be considered. This can be done by 
Table 23. Comparison of $\mathrm{FCl}$ scores between wetlands of the same subclass.

\begin{tabular}{|c|c|c|c|c|c|}
\hline \multirow[b]{2}{*}{ Function } & \multicolumn{3}{|c|}{$\mathrm{FCl}$ scores } & \multirow[b]{2}{*}{ Max score } & \multirow[b]{2}{*}{ Min score } \\
\hline & Site A & Site B & Site C & & \\
\hline Water Storage & 1.0 & 0.6 & 0.2 & 1.0 & 0.2 \\
\hline Cycle Organic Carbon & 0.9 & 0.8 & 0.5 & 0.9 & 0.5 \\
\hline $\begin{array}{l}\text { Maintain a Characteristic Plant } \\
\text { Community }\end{array}$ & 1.0 & 0.7 & 0.4 & 1.0 & 0.4 \\
\hline Provide Characteristic Wildlife Habitat & 0.9 & 0.7 & 0.4 & 0.9 & 0.4 \\
\hline
\end{tabular}

multiplying FCIs by the number of acres impacted to determine Functional Capacity Units (FCUs) for each function (i.e., FCU $=$ FCI $\times$ acres) (Table 24). Using this approach, the relative impact of one project alternative can be compared to other project alternatives by comparing FCUs lost or gained for each alternative (Wakeley et al. 2001).

This method can be used to evaluate the adequacy of a proposed mitigation plan. The goal of no net loss of wetland function requires that project impacts must be completely compensated by the mitigation plan. Project impacts are quantified by calculating FCUs lost at the impact site compared to the project. Benefits of the mitigation plan are quantified by estimating FCUs gained at the mitigation site through enhancement, restoration, or creation of wetlands. As long as the wetlands on the impact site and mitigation site are of the same wetland subclass, the adequacy of the mitigation plan can be determined by comparing FCUs lost to impacts with FCUs gained through mitigation (Wakeley et al. 2001).

The mitigation plan can be adjusted to allow for the time lag associated with restoration of wetlands and associated functions. By estimating the FCUs potentially gained over the course of the mitigation project, the mitigation site can be evaluated for success. Depending on the wetland subclass, the time required to replace lost functions could range from a few years for a herbaceous flat wetland to 50 years or more for a forested riverine wetland. Analyses of this sort can be done readily with software available from the ERDC HGM web page (http://el.erdc.usace.army.mil/wetlands/datanal.html).

\section{Evaluating mitigation plans}

To assess mitigation requirements, it is necessary to first determine baseline FCI values for each assessment area. However, to assess the overall balance between functional losses on the impacted site and 
functional gains on the mitigation site for each mitigation option, it is necessary to compare FCUs between pre-project conditions and postproject conditions for each site and for each project option.

Table 24 shows the assessment of baseline or pre-project conditions on the proposed impact and mitigation sites. Only one mitigation comparison was used in the example in Table 24; however, the methods are the same with more scenarios. In this example the important result of the assessment was that the proposed mitigation site already seemed to function at fairly high levels for most functions, which severely limited the potential gain in function that might be achieved through any of the mitigation plans.

Table 24. Example comparison of mitigation using HGM.

\begin{tabular}{|c|c|c|c|c|c|c|}
\hline \multicolumn{7}{|l|}{ Impact Site } \\
\hline \multirow[b]{2}{*}{ Functions } & \multicolumn{2}{|c|}{ Pre-project } & \multicolumn{2}{|c|}{ Post-project } & \multicolumn{2}{|c|}{ Net loss } \\
\hline & $\mathrm{FCl}$ & FCU & $\mathrm{FCl}$ & FCU & $\mathrm{FCl}$ & FCU \\
\hline Water Storage & 1.0 & 10.0 & 0.2 & 2.0 & -0.8 & -8.0 \\
\hline Cycle Organic Carbon & 0.9 & 9.0 & 0.3 & 3.0 & -0.6 & -6.0 \\
\hline Maintain Characteristic Plant Community & 1.0 & 10.0 & 0.1 & 1.0 & -0.9 & -9.0 \\
\hline Maintain Characteristic Wildlife Habitat & 0.9 & 9.0 & 0.0 & 0.0 & -0.9 & -9.0 \\
\hline Totals & 3.8 & 38 & 0.6 & 6.0 & -3.2 & -32.0 \\
\hline \multirow[t]{2}{*}{ Mitigation Site } & \multicolumn{6}{|c|}{ Acres: 10} \\
\hline & Pre & roject & Pos & project & & gain \\
\hline Functions & $\mathrm{FCl}$ & FCU & $\mathrm{FCl}$ & FCU & $\mathrm{FCl}$ & FCU \\
\hline Water Storage & 0.6 & 6.0 & 0.8 & 8.0 & 0.2 & 2.0 \\
\hline Cycle Organic Carbon & 0.6 & 6.0 & 0.8 & 8.0 & 0.2 & 2.0 \\
\hline Maintain Characteristic Plant Community & 0.6 & 6.0 & 0.8 & 8.0 & 0.2 & 2.0 \\
\hline Maintain Characteristic Wildlife Habitat & 0.6 & 6.0 & 0.8 & 8.0 & 0.2 & 2.0 \\
\hline Totals & 2.4 & 24.0 & 6.0 & 32.0 & 0.8 & 8.0 \\
\hline Impact and mitigation total FCUs: & \multicolumn{6}{|c|}{-24.00} \\
\hline
\end{tabular}

In this example, the mitigation site already exhibits a high level of function. It is not possible to achieve an adequate increase in function for any of the variables or the total of all of the functions to achieve a "no net loss" of function. Possible solutions would be to increase the acreage of the 
mitigation site, reduce the acreage of the impact, or both. Other examples of mitigation assessments using HGM are presented by Hauer and Smith (1998).

There are a variety of ways to interpret the result of FCUs for the purposes of mitigation. Basing mitigation requirements on the replacement of functional loss for each function would most closely address the concept "no net loss" of wetland function. However, this method is the most difficult to apply and monitor for success. Other methods of interpreting mitigation requirements are explained in the following paragraphs.

\section{Total FCUs}

The example in Table 23 presents the possibility of comparing the difference in each functional score and the total FCUs. Using the total FCU in planning mitigation requirements has the potential of having one or two functions dominate the mitigation and restoration at the expense of other functions. However, using the total loss in FCUs is a practical approach if purchasing credits from a mitigation bank or in-lieu fee program.

\section{Average}

Averaging FCUs for all functions is one of the most common approaches to applying results of HGM assessments to determining mitigation requirements. In cases where each FCI does not vary by more than 0.1 from the other functions, the result is not likely to have much impact on mitigation requirements. A potential problem arises when the differences between FCI values for one or two functions are 0.5 or more than the other functions. This could potentially reduce the amount of mitigation necessary to compensate for the wetland loss.

\section{Lowest FCl score}

Another approach for determining mitigation requirements is to use the lowest FCI score for all of the functions. In the example in Table 23, the project had the greatest impact to "Maintain Characteristic Plant Community" and "Maintain Characteristic Wildlife Habitat," with a loss in function of 0.9. The logic is that to achieve "no net loss" in function, the greatest functional loss must be compensated. 


\section{Monitor mitigation}

Monitoring of wetland mitigation is required as part of any mitigation plan (U.S. Department of Defense 2008). HGM guidebooks can be used to monitor mitigation success (Wakeley 1999). Mitigation often takes many years to realize the full potential of the restoration. For example, forested systems may take 50 years or more to reach maturity. FCIs can be tracked over time to evaluate if target goals for each function are being met or if the mitigation plan needs to be reevaluated to increase the chance of success. In some cases monitoring individual variables may be more useful than the overall FCI score for a specific function. It should, for example, be possible to restore hydrology variables relatively quickly if restoration is possible. If hydrology is not restored quickly, then the success of restoring other variables, such as vegetation structure, is not likely.

\section{Summary}

Assessments using HGM Guidebooks help to reduce the subjectivity involved in evaluating project impacts and mitigation plans. This helps to ensure that every permit application is evaluated fairly and objectively, and reduces the reliance on subjective evaluation methods or mitigation formulas established by precedent or interagency agreement rather than by impartial analysis.

On the other hand, the HGM approach cannot be used to compare wetlands of different types, such as pine savannas and bottomland hardwoods, because the models, variables, and reference standards are different. In addition, the HGM approach separately assesses each function, but does not address trade-offs, such as when losses of one function are mitigated through gains in another function. None of these approaches to determining mitigation requirements addresses the issues of temporal loss of wetland function or determining mitigation ratios for off-setting temporal loss. Specific restoration trajectories for wetland restoration were developed to address temporal loss of wetland functions (see, for example, HGM Guidebooks produced for Arkansas and the Yazoo Basin of Mississippi (http://el.erdc.usace.army.mil/wetlands/guidebooks.cfm). Methods for using trajectories in mitigation planning are discussed in Klimas (2006).

The HGM Approach cannot substitute for knowledge and experience when planning wetland mitigation. For example, an evaluation of mitigation 
options requires predicting future conditions on restored wetlands and understanding restoration failure rates, which should be based on experience with similar projects in the same wetland subclass. The goal of the HGM Approach is to provide sound technical input into a decision based also on regulations, policy, and common sense. Individual Corps Districts have the discretion to set mitigation ratios. 


\section{References}

Adamus, P. R. 1992. Part IV: Wetland data sources and collection methods. In State Wetland Strategies: A guide to protection and managing the resource. World Wildlife Fund. Washington, DC: Island Press.

Adamus, P., and K. Brandt. 1990. Impacts on quality of inland wetlands of the United States: A survey of indicators, techniques, and applications of community-level biomonitoring data. Corvallis, OR: Environmental Research Lab, Environmental Protection Agency.

Adamus, P. R. 1983. A Method for Wetland Functional Assessment: Volume II FHWA Assessment method.

Adamus, P. R, E. J. Clairain, Jr., R. D. Smith, and R. E. Young. 1987. Wetland Evaluation Technique (WET), Volume 2: Methodology (Operational Draft Report). Vicksburg, MS: U.S. Army Engineer Waterways Experiment Station.

Adamus, P. R. 1995. Validating a habitat evaluation method for predicting avian richness. Wildlife Society Bulletin 23 (4):743-749.

Ainslie, W. B. 1994. Rapid Wetland Functional Assessment - Its Role and Utility in the Regulatory Arena. Water Air and Soil Pollution 77 (3-4):433-444.

Ainslie, W. B., R. D. Smith, B. A. Pruitt, T. H. Roberts, E. J. Sparks, L. West, G. L. Godshalk, and M. V. Miller. 1999. A Regional Guidebook for Assessing the Functions of Low Gradient Riverine Wetlands in Western Kentucky. Vicksburg, MS: US Army Engineers Research and Development Center.

Ammann, A. P., R. W. Franzen, and J. L. Johnson. 1986. Method for the evaluation of nontidal wetlands in Connecticut. Bulletin No. 9. Concord, NH: Connecticut Department of Environmental Protection and USDA Soil Conservation Service.

Ammann, A. P., and A. L. Stone. 1991. Method for the comparative evaluation of nontidal wetlands in New Hampshire. New Hampshire Dept. of Environmental Services.

Apfelbeck, R., and E. Farris. 2005. Montana Wetland Rapid Assessment Method Guidebook (Version 2.0). Helena, MT: Montana Department of Environmental Quality; Planning, Prevention, and Assistance Division; Water Quality Planning Bureau; Wetland Section.

Arrhenius, O. 1921. Species and area. J ournal of Ecology 9:95-99.

Bailey, R. G. 1980. Description of the ecoregions of the United States. U.S. Department of Agriculture Miscellaneous Publication 1391, 77. Washington, DC.

Bailey, R. G., P. E. Avers, T. King, and W. H. McNab. 1994. Ecoregions and subregions of the United States (map). Washington, DC: US Geological Survey.Scale 1 $(7,500,000)$. 
Baker, J. P., W. J. Warrenhicks, J. Gallagher, and S. W. Christensen. 1993. Fish population Losses from Adirondack Lakes - the role of surface-water acidity and acidification. Water Resources Research 29 (4):861-874.

Barbour, M. T., J. B. Stribling, and J. R. Karr. 1995. Multimetric approach for establishing biocriteria and measuring biological condition. In Biological assessment and criteria: Tools for water resource planning and decision making, ed. W.S. Davis and T.P. Simpson, 63-77. Boca Raton, FL: Lewis Publishers.

Bartoldus, C. C., E. W. Garbisch, and M. L. Kraus. 1994. Evaluation for planned wetlands (EPW): A procedure for assessing wetland functions and a guide to functional design. Environmental Concern. St. Michaels, MD.

Bartoldus, C. C. 1999. A comprehensive review of wetland assessment procedures: A guide for wetland practioners. St. Michaels, MD: Environmental Concerns, Inc.

Bauder, E. T., A. J. Bohonak, B. Hecht, M. A. Simovich, D. Shaw, D. G. Jenkins, and M. Rains. 2009. A Draft Regional Guidebook for applying the hydrogeomorphic approach to assessing wetland functions of vernal pool depressional wetlands in southern California. San Diego, CA: San Diego State University.

Bender, M. E. and D. L. Correll. 1974. The use of wetlands as nutrient removal systems. Publication No. 29. Baltimore, MD: Chesapeake Research Consortium.

Berkowitz, J., A. F. Casper, and C. Noble. 2011. A multiple watershed field test of hydrogeomorphic functional assessment of headwater streams--Variability in field measurements between independent teams. Ecological Indicators 11 (5):1472-1475.

Best, L. B., and D. F. Stauffer. 1986. Factors confounding evaluation of bird-habitat relationships. (Journal Article).

Biggs, B. J. F., M. J. Duncan, I. G. Jowett, J. M. Quinn, C. W. Hickey, R. J. Davies-Colley, and M. E. Close. 1990. Ecological characterisation, classification, and modelling of New Zealand rivers: An introduction and synthesis. New Zealand J ournal of Marine and Freshwater Research 24 (3):277-304.

Block, W. M., M. L. Morrison, J. Verner, and P. N. Manley. 1994. Assessing wildlifehabitat-relationships models: A case study with California oak woodlands. Wildlife Society Bulletin 22 (4):549-561.

Bonham, C. D. 1989. Measurements for terrestrial vegetation: Wiley-Interscience.

Bormann, F. H., and Likens, G. E. 1969. The watershed-ecosystem concept and studies of nutrient cycling. In The ecosystem concept in natural resources management, ed. G. MI VanDyne, 49-76. New York: Academic Press.

Brinson, M. M. 1993. A hydrogeomorphic classification for wetlands. Vicksburg, MS: U.S. Army Engineer Waterways Experiment Station.

Brinson, M. M. 1995. The hydrogeomorphic approach explained. National Wetlands Newsletter, November/December. 
Brinson, M. M., and R. Rheinhardt. 1996. The role of reference wetlands in functional assessment and mitigation. Ecological Application 6 (1):69-76.

Brinson, M. M., F.R. Hauer, L. C. Lee, W. L. Nutter, R. D. Rheinhardt, R. D. Smith, and D. Whigham. 1995. A guidebook for application of hydrogeomorphic assessments to riverine wetlands. Vicksburg, MS: U.S. Army Engineer Waterways Experiment Station.

Brower, J. E., J. H. Zar, and C. N. Von Ende. 1997. Field and laboratory methods for general ecology. Dubuque, IA: WCB/McGraw Hill.

Brunner, G. W. 1988. Comparison of modeling techniques for wetland areas. Project Report 88-4. Davis, CA: U.S. Army Corps of Engineers Hydrologic Engineering Center.

Bryce, S. A., J. M. Omernik, and D. P. Larsen. 1999. Ecoregions: A geographic framework to guide characterization and ecosystem management. Environmental Practice 1:141-155.

Cain, S. A. 1938. The species-area curve. American Midland Naturalist 19 (3):573-581.

Canter, L. W., and L. G. Hill. 1979. Handbook of variables for environmental impact assessment. Ann Arbor Science.

Carroll, R., J. Tracy, and G. Pohll. 2000. Numerical assessment of hyrogeomorphic wetland functions. Phase II. Publication No. 41166. Reno, NV: Desert Research Institute, Division of Hydrologic Sciences.

Caswell, H. 1976. The validation problem. In Systems analysis and simulation in ecology, Vol. IV, ed. B. C. Patten, 313-325. New York: Academic Press.

Chescheir, G., J. Gilliam, R. Skaggs, and R. Broadhead. 1991. Nutrient and sediment removal in forested wetlands receiving pumped agricultural drainage water. Wetlands 11 (1):87-103.

Chescheir, G. M., R. W. Skaggs, J. W. Gilliam, and R. G. Broadhead. 1987. The hydrology of wetland buffer areas for pumped agricultural drainage water. In The Ecology and Management of Wetlands: Beckenham, England: Croom Helm.

Clairain, E. J., Jr. 2002. Hydrogeomorphic approach to assessing wetland functions: Guidelines for developing regional guidebooks. Chapter 1, Introduction and overview of the hydrogeomorphic approach. ERDC/EL TR-02-3. Vicksburg, MS: U.S. Army Engineer Research and Development Center.

Commission for Environmental Cooperation (CEC). 1997. Ecological regions of North America: Toward a common perspective. Quebec, Canada: Commission for Environmental Cooperation.

Condit, R., S. P. Hubbell, J. V. Lafrankie, R. Sukumar, N. Manokaran, R. B. Foster, and P. S. Ashton. 1996. Species-area and species-individual relationships for tropical trees: A comparison of three 50-ha plots. J ournal of Ecology 84 (4):549-562. 
Conservation Foundation. 1988. Protecting America's wetlands: An action agenda. The Final Report of the National Wetlands Policy Forum. Washington, DC: The Conservation Foundation.

Cook, J. G., and L. L. Irwin. 1985. Validation and modification of a habitat suitability model for pronghorns. Wildlife Society Bulletin 13 (4):440-448.

Cowardin, L. M. 1979. Classification of wetlands \& deepwater habitats of the US. DIANE Publishing.

Dahl, T. E. 1990. Wetlands losses in the United States, 1780's to 1980's.Washington, DC: U.S. Department of the Interior, Fish and Wildlife Service. Jamestown, ND: Northern Prairie Wildlife Research Center: http://www.hpwrc.usgs.gov/resource/ wetlands/wetloss/index.htm (Version 16JUL97).

Dahl, T. E. 2000. Status and trendsof wetlands in conterminousUnited States 1986 to 1997. Washington, DC: U.S. Department of the Interior, Fish and Wildlife Service.

Dahl, T. E. 2005. Status and trends of wetlands in the conterminous United States 1998 to 2004. U.S. Dept. of the Interior, US Fish and Wildlife Service.

Enviromental Laboratory. 1987. Corps of Engineers Wetlands Delineation Manual. Technical Report Y-87-1. Vicksburg, MS: U.S. Army Engineer Waterways Experiment Station.

Euler, D. L., J. F. T. Carreiro, G. B. McCullough, E. A. Snell, V. Glooschenko, and R. H. Spurr. 1983. An evaluation system for wetlands of Ontario south of the Precambrian Shield. Ontario, Canada: Ontario Ministry of Natural Resources and Canadian Wildlife Service.

Faulkner, S.P., W.H. Patrick, Jr., and R.P. Gambrell. 1989. Field techniques for measuring wetland soil parameters. Soil Sci Soc AmJ 53 (3):883-890.

Feeney, P., and B. Morrell. 1985. Wetlands disposal is sewage option. American City and County 100:91-92.

Fenneman, N. M., and D. W. Johnson. 1946. Physical divisions of the United States: US Geol. Survey map 1.

Fennessy, M., A. Jacobs, and M. Kentula. 2007. An evaluation of rapid methods for assessing the ecological condition of wetlands. Wetlands 27 (3):543-560.

Fennessy, M. S., A. D. Jacobs, and M. E. Kentula. 2004. Review of rapid methods for assessing wetland condition. US Environmental Protection Agency: Washington, DC EPA/620/R-04/ 009.(Last visited April 25, 2011.) http://epa.gov/wed/pages/publications/authored/EPA620R-04009Fennessy RapidMethodReview.pdf (Journal Article).

Fritz, W. R., and S. C. Helle. 1979. Cypress wetlands - natural tertiary treatment alternative. Water \& Sewage Works 126(4):58-60. 
Gallant, A. L., T. R. Whittier, D. P. Larsen, J. M. Omernik, and R. M. Hughes. 1989. Regionalization as a tool for managing environmental resources. EPA-600-389-060. Corvallis, OR: U. S. Environmental Protection Agency, Office of Research and Development.

Golet, F. C., and J. S. Larson. 1974. Classification of freshwater wetlands in the glaciated Northeast. Bureau of Sport Fisheries and Wildlife, Fish and Wildlife Service, US Dept. of the Interior.

Gorham, E. 1994. The future of research in Canadian peatlands: A brief survey with particular reference to global change. Wetlands 14(3):206-215.

Graf, W. L. 1987. Geomorphic systems of North America. Vol. 2: Geological Society of Amer.

Green, R. H. 1979. Sampling design and statistical methods for environmental biologists. John Wiley \& Sons, Inc.

Greeson, P. E., J. R. Clark, and J. E. Clark. 1979. Wetland functions and values: The state of our understanding. Paper read at Technical publication series-American Water Resources Association, Nov. 6-10, 1978, Orlando, FL.

Guertin, D. P., P. K. Barten, and K. N. Brooks. 1987. The peatland hydrologic impact model: Development and testing. Nordic Hydrology 18 (2):79-100.

Gunderson, L. H. 1989. Historical hydropatterns in wetland communities of Everglades National Park. Freshwater wetlands and wildlife 61:1099-1111.

Hall, C. A. S., and J. W. Day, Jr., ed. 1977. Systems and models: Terms and basic principles, Ecosystem Modeling in Theory and Practice: An Introduction with Case Histories. New York: Wiley.

Hammer, D. E., and R. H. Kadlec. 1986. A model for wetland surface water dynamics. Water Resources Research 22 (13):1951-1958.

Hauer, R. R., B. J. Cook, M. C. Gilbert, Jr., E. J. Clairain, and R. D. Smith. 2002. A Regional Guidebook for Applying the Hydrogeomorphic Approach to Assessing Wetland Functions of Intermontane Prarie Pothole Wetlands in the Northern Rocky Mountains. Vicksburg, MS: U.S. Army Engineer Research and Development Center.

Hauer, F. R., and R. D. Smith. 1998. The hydrogeomorphic approach to functional assessment of riparian wetlands: evaluating impacts and mitigation on river floodplains in the U.S.A. Freshwater Biology 40 (3):517-530.

Haukos, D. A., and L. M. Smith. 1993. Moist-soil management of playa lakes for migrating and wintering ducks. Wildlife Society Bulletin 21 (3):288-298.

Heliotis, F. D., and C. B. DeWitt. 1987. Rapid water Table responses to rainfall in a northern peatland ecosystem1. JAWRAJ ournal of the American Water Resources Association 23 (6):1011-1016.

Hill, A. J., V. S. Neary, and K. L. Morgan. 2006. Hydrologic modeling as a development tool for HGM functional assessment models. Wetlands 26 (1):161-180. 
Hollands, G. G. 1987. Hydrogeologic classification of wetlands in glaciated regions. In Wetland Hydrology, Proceedings from a national wetland symposium, ed., J. Kusler, 26-30. Berne, NY: Association of State Wetland Managers, Inc.

Hollands, G. G., and D. W. Magee. 1985. A method for assessing the functions of wetlands. In Proceedings of the National Wetland Assessment Symposium, ed. J. Kusler and P. Riexinger. Berne, NY: Association of State Wetland Managers.

Hruby, T. 1997. Continuing the discussion: Scientific and technical issues regarding the Hydrogeomorphic Approach to function assessment of wetlands. Society of Wetland Scientists Bulletin 14:23-24.

Hubbard, D. E. 1988. Glaciated prairie wetland functions and values: A synthesis of the literature. Biological Report 88/43. Washington, DC: U.S. Fish and Wildlife Service.

Hughes, R. M. 1995. Defining accepTable biological status by comparing with reference conditions. In Biological assessment and criteria: tools for water resource planning and decision making. Boca Raton, FL: CRC Press: 31-48.

Hughes, R. M., and R. F. Noss. 1992. Biological diversity and biological integrity: Current concerns for lakes and streams. Fisheries 17 (3):11-19.

Hughes, R. M., C. B. Johnson, S. S. Dixit, A. T. Herlihy, P. R. Kaufmann, W. L. Kinney, D. P. Larsen, P. A. Lewis, D. M. McMullen, and A. K. Moors. 1993. Development of lake condition indicators for EMAP-1991 pilot. In EMAP-Surface waters 1991 pilot report: 7-90.

Hughes, R. M., D. P. Larsen, and J. M. Omernik. 1986. Regional reference sites: A method for assessing stream potentials. Environmental Management 10 (5):629635 .

Hughes, R. M., P. R. Kaufmann, A. T. Herlihy, T. M. Kincaid, L. Reynolds, and D. P. Larsen. 1998. A process for developing and evaluating indices of fish assemblage integrity. Canadian J ournal of Fisheries and Aquatic Sciences 55 (7):1618-1631.

Inhaber, H. 1976. Environmental indices. John Wiley.

Jorgensen, S. 1988. Fundamentals of ecological modeling. Amsterdam, The Netherlands: Elsevier.

Kadlec, R. H. 1988. Monitoring wetland responses. In Increasing our wetland resources, ed. J. Zelazny and J. S. Feierabend, 114-120. Washington, DC: National Wildlife Federation.

Kadlec, R. H., D. L. Tilton, and K. C. Ewel. 1979. The use of freshwater wetlands as a tertiary wastewater treatment alternative. C R C Critical Reviews in Environmental Control 9 (2):185-212.

Karr, J. R., E. W. Chu, and U.S. Environmental Protection Agency. 1997. Biological monitoring and assessment: Using multimetric indexes effectively. University of Washington. 
Keeler-Wolf, T., D. R. Elam, K. Lewis, and S. A. Flint. 1998. California vernal pool assessment - Preliminary Report. California Department of Fish and Game.

Kent, M., and P. Coker. 1992. Vegetation description and analysis: A practical approach. New York: Wiley.

King, S. L., and J. A. Allen. 1996. Plant succession and greentree reservoir management: Implications for management and restoration of bottomland hardwood wetlands. Wetlands 16(4):503-511.

Kleindl, W., M. C. Rains, and F. R. Hauer. 2010. HGM is a rapid assessment: Clearing the confustion. Wetland Science and Practice 27(3): 17-22.

Kleiss, B. 1996. Sediment retention in a bottomland hardwood wetland in Eastern Arkansas. Wetlands 16 (3):321-333.

Klemm, D. J., K. A. Blocksom, F. A. Fulk, A. T. Herlihy, R. M. Hughes, P. R. Kaufmann, D. V. Peck, J. L. Stoddard, W. T. Thoeny, M. B. Griffith, and W. S. Davis. 2003. Development and evaluation of a macroinvertebrate biotic integrity index (mbii) for regionally assessing mid-atlantic highlands streams. Environmental Management 31 (5):0656-0669.

Klimas, C. 2006. Development and application of functional recovery trajectories for wetland restoration. Washington, D.C.: U.S. Environmental Protection Agency, Office of Wetlands, Oceans and Watersheds, Wetlands Division.

Klimas, C.V., E.O. Murray, H. Langston, J. Pagan, T. Witsell, and T. Foti. 2008. A Regional Guidebook for conducting functional assessments of forested wetlands in the Arkansas Valley Region of Arkansas. ERDC/EL TR-o8-23. Vicksburg, MS: U.S. Army Engineer Research and Development Center.

Klimas, C. V., E. O. Murray, J. Pagan, H. Langston, and T. Foti. 2004. A Regional Guidebook for applying the hydrogeomorphic approach to assessing wetland functions of forested wetlands in the Delta Region of Arkansas, Lower Mississippi River Alluvial Valley. ERDC/EL TR-04-16. Vicksburg, MS: U.S. Army Engineer Research and Development Center.

Klimas, C. V., E. O. Murray, J. Pagan, H. Langston, and T. Foti. 2005. A Regional Guidebook for applying the Hydrogeomorphic Approach to assessing wetland functions of forested wetlands in the West Gulf Coastal Plain Region of Arkansas. ERDC/EL TR-05-12. Vicksburg, MS: U.S. Army Engineer Research and Development Center.

Klimas, C. V., E. O. Murray, J. Pagan, H. Langston, T. Witsell, and T. Foti. 2006. A Regional Guidebook for conducting functional assessments of wetland and riparian forests in the Ouachita Mountains and Crowley's Ridge Regions of Arkansas. ERDC/EL TR-06-14. Vicksburg, MS: U.S. Army Engineer Research and Development Center.

Klimas, C. 2006. Development and application of functional recovery trajectories for wetland restoration. 4W-0316-NASX. Washington, D.C. 
Klimas, C. V., R. D. Smith, R. T. Saucier, and J. Raasch. 2005. Hydrogeomorphic classification of forested wetlands in the lower Mississippi Valley: Implications for restoration and management. In Ecology and management of bottomland hardwood systems: The state of our understanding, ed. L. H. Fredrickson, S. L. King, and R. M. Kaminski. University of Missouri-Columbia, Gaylord Memorial Laboratory Special Publication No. 10, 77-91.

Küchler, A. W. 1964. Potential natural vegetation of the conterminous United States. Special Publication 36. New York: American Geographical Society.

Küchler, A. W. 1970. Potential Natural Vegetation Map (scale 1:7,500.000). In The National Atlas of the United States of America. Washington, DC: U.S. Geological Survey.

LaBaugh, J. W. 1986. Wetland ecosystem studies from a hydrologic perspective1. JAWRA J ournal of the American Water Resources Association 22 (1):1-10.

Lancia, R. A., S. D. Miller, D. A. Adams, and D. W. Hazel. 1982. Validating habitat quality assessment: An example. Paper read at Trans. North Am. Wildl. and Nat. Resour. Conf, 1982.

Landers, J. 1991. Use of wetlands for water quality improvement under the U.S. Environmental Protection Agency. Region V Clean Lakes Program. Environmental Management 15:151-62.

Larson, J. S. 1976. Models for assessment of freshwater wetlands. University of Massachusetts, Water Resources Research Center.

Leibowitz, S. 2003. Isolated wetlands and their functions: An ecological perspective. Wetlands 23 (3):517-531.

Lemly, J. M., and J. Rocchio. Resources Colorado, Dept. of Natural, Wildlife Colorado. Division of, Viii United States. Environmental Protection Agency. Region, and Program Colorado Natural Heritage. 2009. Vegetation Index of Biotic Integrity (VIBI) for Headwater Wetlands in the Southern Rocky Mountain: Version 2.0: Calibration of Selected VIBI Models. Colorado State University, Colorado Natural Heritage Program.

Loftis, J. C., R. C. Ward, R. D. Phillips, and C. H. Taylor. 1989. An evaluation of trend detection techniques for use in water-quality monitoring programs. EPA-600-389-037. Corvallis, OR: U.S. Environmental Protection Agency, Office of Research and Development.

Lonard, R. I., E. T. Clairain, R. T. Huffman, J. W. Hardy, C. D. Brown, P. E. Ballard, and J. W. Watts. 1981. Analysis of methodologies used for the assessment of wetlands values. Prepared by the U.S. Army Engineer Waterways Experiment Station for the U.S. Water Resources Council, Washington, DC.

Lyon, J. G., and R. G. Greene. 1992. Use of aerial photographs to measure the historical areal extent of Lake Erie coastal wetlands. Photogrammetric Engineering and Remote Sensing 58 (9):1355-1360.

Marcot, B. G. 1983. Monitoring wildlife habitat and validation of wildlife-habitat relationships models. 
McCormick, F. H., R. M. Hughes, P. R. Kaufmann, D. V. Peck, J. L. Stoddard, and A. T. Herlihy. 2001. Development of an index of biotic integrity for the Mid-Atlantic Highlands region. Transactions of the American Fisheries Society 130 (5):857877 .

Mitsch, W. J., and J.G. Gosselink. 200o. Wetlands. 3rd ed. New York, NY: John Wiley \& Sons.

Mitsch, W. J., M. Straskraba, and S. E. Jorgensen. 1988. Wetland modelling. In Developments in Environmental Modelling: Elsevier Science Publications.

Mueller-Dombois, D., and H. Ellenberg. 1974. Aims and methods of vegetation ecology. New York: John Wiley and Sons.

Natural Resources Conservation Service. 1999. Stream visual assessment protocol. National Water and Climate Center Technical Note 99-1.

Natural Resources Conservation Service. 2004. Riparian Assessment: Using the NRCS Riparian Assessment Method.

Nature Conservancy. 2005. Indicators of Hydrologic Alteration User's Manual, Version 7. The Nature Conservancy with Smythe Scientific Software and Totten Software Design.

Noble, C. V., R. Evans, M. McGuire, K. Trott, M. Davis, and E. J. Clairain, Jr. 2002. A Regional Guidebook for Applying the Hydrogeomorphic Approach to Assessing Wetland Functions of Flats Wetlands in the Everglades. ERDC/EL TR-02-19. Vicksburg, MS: U.S. Army Engineer Research and Development Center.

Noble, C. V., J. S. Wakeley, T. H. Roberts, and C. Henderson. 2007. Regional for applying the hydrogeomorphic approach to assessing the functions of headwater slope wetlands on the Mississippi and Alabama Coastal Plains. ERDC/EL TR-07-9. Vicksburg, MS: U.S. Army Engineer Research and Development Center.

Noble, C. V., J. S. Wakeley, T. H. Roberts, and C. Henderson. 2007. A Regional Guidebook for Applying the Hydrogeomorphic Approach to Assessing the Functions of Headwater Slope Wetlands on the Mississippi and Alabama Coastal Plains. ERDC/EL TR-07-9. Vicksburg, MS.

Novitzki, R. P., R. D. Smith, and J. D. Fretwell. 1997. Restoration, creation and recovery of wetlands: Wetland functions, values and assessment. US Geological Survey National Water Summary on Wetland Resources.USGS Water Supply Paper 2425 .

O'Banion, K. 1980. Use of value functions in environmental decisions. Environmental Management 4 (1):3-6.

Olden, J. D., and N. L. Poff. 2003. Redundancy and the choice of hydrologic indices for characterizing streamflow regimes. River Research and Applications 19 (2):101121.

Omernik, J. M. 1987. Ecoregions of the conterminous United States. Annals of the Association of American Geographers 77 (1):118-125. 
Omernik, J. M. 1995. Ecoregions: A spatial framework for environmental management. In Biological assessment and criteria: Tools for water resource planning and decision making, ed. W.S. Davis and T.P. Simon, 49-62. Boca Raton, FL: Lewis Publishers.

Omernik, J. M. 2004. Perspectives on the nature and definition of ecological regions. Environmental Management 34 (o):S27-S38.

Omernik, J. M., and A. L. Gallant. 1990. Defining regions for evaluating environmental resources. In Global natural resource monitoring and assessments: Preparing for the 21st Century, coordinators H. G. Lund and G. Reto, 936-947. Bethesda, MD: American Society of Photogrammetry and Remote Sensing.

O’Neil, L. J. 1993. Test and modification of a Northern Bobwhite habitat suitability index model. Technical Report EL-93-5. Vicksburg, MS: U.S. Army Engineer Waterways Experiment Station.

O'Neil, L. J., T. H. Roberts, J. S. Wakeley, and J. W. Teaford. 1988. A Procedure to modify habitat suitability index models. Wildlife Society Bulletin 16 (1):33-36.

Ott, W.R. 1978. Environmental indices: theory and practice.

Overton, W. S. 1977. A strategy of model construction. Ecosystem modeling in theory and practice:49-73.

Payne, N. F. 1998. Wildlife habitat management of wetlands. Krieger.

Pohll, G., J. Tracy, and R. Smith. 1999. Numerical assessment of hydrogeomorphic wetland functions. Paper read at Wetlands \& Remediation: An International Conference, 1999.

Raich, J. W., and W. H. Schlesinger. 1992. The global carbon dioxide flux in soil respiration and its relationship to vegetation and climate. Tellus B 44 (2):81-99.

Rheinhardt, R. D., M. M. Brinson, R. R. Christian, K. H. Miller, and G. F. Meyer. 2007. A reference-based framework for evaluating the ecological condition of stream networks in small watersheds. Wetlands 27(3):524-542.

Rheinhardt, R. D., M. C. Rheinhardt, and M. M. Brinson. 2002. A Regional Guidebook for Applying the Hydrogeomorphic Approach to Assessing Wetland Functions of Wet Pine Flats on Mineral Soils in the Atlantic and Gulf Coastal Plains. ERDC/EL TR-02-9. Vicksburg, MS: U.S. Army Engineer Research and Development Center.

Rheinhardt, R., M. Brinson, and P. Farley. 1997. Applying wetland reference data to functional assessment, mitigation, and restoration. Wetlands 17 (2):195-215.

Richter, B. D., J. V. Baumgartner, J. Powell, and D. P. Braun. 1996. A method for assessing hydrologic alteration within ecosystems. Conservation Biology 10 (4):1163-1174. 
Rocchio, J. 2007. Assessing Ecological Condition of Headwater Wetlands in the Southern Rocky Mountain Ecoregion using a vegetation index of biotic integrity: (Version 1.0). Unpublished report prepared for Colorado Department of Natural Resources and U.S. Environmental Protection Agency Region VIII. Fort Collins, CO: Colorado State University, Colorado Natural Heritage Program.

Rosenberry, D. O. 1990. Inexpensive groundwater monitoring methods for determining hydrologic budgets of lakes and wetlands. Paper read at National Conference on Enhancing the States' Lake and Wetland Management Programs. U.S.

Environmental Protection Agency, North American Lake Management Society.

Rykiel, E. J., Jr. 1996. Testing ecological models: the meaning of validation. Ecological Modelling 90 (3):229-244.

SAS Institute, Inc. 1988. SAS Language Guide for Personal Computers, Release 6.03 Edition. Cary, NC.

Sather, J. H., and R. D. Smith. 1984. An overview of major wetland functions and values. Vol. 18: The Team. Washington, DC: U.S. Department of the Interior, Fish and Wildlife Service.

Saucier, R. 1995. Quaternary Deposits of the Lower Mississippi River Valley. U.S. Army Engineer Waterways Experiment Station.

Schamberger, M. L., and L. J. O'Neil. 1986. Concepts and constraints of habitat-model testing. In Wildlife 2000: Modeling habitat relationships of terrestrial vertebrates, ed. J. Vedrner, M. L. Morrison, and C. J. Ralph, 5-10. Madison, WI: University of Wisconsin Press.

Schroeder, R. L., and S. L. Haire. 1993. Guidelines for the development of community level habitat evaluation models. Biological report.U.S.Fish and Wildlife Service 8:8.

Schuster, E. G., and H. R. Zuuring. 1986. Quantifying the unquantifiable: Or, have you stopped abusing measurement scales? J ournal of Forestry 84.

Sedell, J. R., and J. L. Froggatt. 1984. Importance of streamside forests to large rivers: The isolation of the Willamette River, Oregon, USA, from its floodplain by snagging and streamside forest removal. Verh.Internat.Verein.Limnol 22:18281834 .

Sedell, J. R., and K. J. Luckessa. 1981. Using the historical record as an aid to salmonid habitat enhancement. U.S. Forest Service.

Shafer, D. J., T. H. Roberts, M.S. Peterson, and K. Schmid. 2007. A Regional Guidebook for applying the Hydrogeomorphic Approach to assessing the functions of tidal fringe wetlands along the Mississippi and Alabama Gulf Coast. ERDC/EL TR07-2. Vicksburg, MS: US Army Engineer Research and Development Center.

Shafer, D. J., and D. J Yozzo. 1998. National guidebook for application of hydrogeomorphic assessment to tidal fringe wetlands. Technical Report WRPDE-16. Vicksburg, MS: U.S. Army Engineer Waterways Experiment Station. 
Simon, T. P. 1995. Biological assessment and criteria: tools for water resource planning and decision making. CRC Press.

Smith, P. G. R., and J. B. Theberge. 1986. A review of criteria for evaluating natural areas. Environmental Management 10 (6):715-734.

Smith, P. G. R., and J. B. Theberge. 1987. Evaluating natural areas using multiple criteria: Theory and practice. Environmental Management 11 (4):447-460.

Smith, R. D. 1993. A Conceptual framework for assessing the functions of wetlands. Technical Report WRP-DE-3. Vicksburg, MS: U.S. Army Engineer Waterways Experiment Station.

Smith, R. D., A. Amman, C. Bartoldus, and M. M. Brinson. 1995. An approach for assessing wetland functions using hydrogeomorphic classification, reference wetlands, and functional indices. Technical Report WRP-DE-9. Vicksburg, MS: U.S. Army Engineer Waterways Experiment Station.

Smith, R. D., and J. S. Wakeley. 2001. Hydrogeomorphic approach to assessing wetland functions: Guidelines for developing regional guidebooks; Chapter 4 Developing assessment models. ERDC/EL TR-01-30. Vicksburg, MS: U.S. Army Engineer Research and Development Center.

Smith, R. D., and C.V. Klimas. 2002. A regional guidebook for applying the hydrogeomorphic approach to assessing wetland functions of selected regional wetland subclasses, Yazoo Basin, Lower Mississippi River Alluvial Valley. ERDC/EL TR-02-4. Vicksburg, MS: U.S. Army Engineer Research and Development Center

Solomon, R. C., and N. R. Sexton. 1994. Methods for evaluating wetland functions. Wetlands Research Program Technical Note WG-EV-2.2. Vicksburg, MS: U.S. Army Engineer Waterways Experiment Station.

Stewart, R. E., and H. A. Kantrud. 1971. Classification of natural ponds and lakes in the glaciated prairie region. Resource Publication 92.

Stoddard, J. L., D. P. Larsen, C. P. Hawkins, R. K. Johnson, and R. H. Norris. 2006. Setting expectations for the ecological condition of streams: The concept of reference condition. Ecological Applications 16 (4):1267-1276.

Tiedje, J. M. 1982. Denitrification. In Methods of soil analysis; Part 2,chemical and microbiological properties, 1011-1026. Agronomy Monograph No. 9. Madison, WI: ASA-SSSA.

Tiedje, J. M., J. Sørensen, and Y. Y. L. Chang. 1981. Assimilatory and dissimilatory nitrate reduction: Perspectives and methodology for simultaneous measurement of several nitrogen cycle processes. Ecological Bulletins (Sweden).

Trautman, M. B. 1981. The fishes of Ohio: Columbus. Ohio, State University Presses.

Trewartha, G. T. 1943. An introduction to weather and climate. $2^{\text {nd }}$ ed. New York: McGraw-Hill. 
U.S. Army Corps of Engineers (USACE). 2010. Regional Internet Bank Information Tracking System (RIBITS).

U.S. Army Engineer District, Charleston. 2002. Compensatory Mitigation - Standard Operating Procedures.

U.S. Department of Agriculture (USDA)/Natural Resources Conservation Service (NRCS). 2006. Land resource regions and major land resource areas of the United States, the Caribbean, and the Pacific Basin. Agriculture Handbook 296. Washington, DC. http://soils.usda.gov/survey/geography/mlra/

U.S. Department of Agriculture (USDA)/Natural Resources Conservation Service (NRCS). 2003. National range and pasture handbook. Grazing Lands Technology Institute.

U.S. Department of Defense. 2008. Compensatory mitigation for losses of aquatic resources; Final Rule. Federal Register (33 CFR Parts 325 and 332 and 40 CRF Part 230. Washington,DC.

U.S. Environmental Protection Agency (USEPA). 1984. Literature review of wetland evaluation methodologies. Chicago, IL: EPA Region 5.

U.S. Environmental Protection Agency (USEPA). 2002a. Methods for evaluating wetland conditions: \#1 Introduction to wetland biological assessment. EPA-822-R-02014. Washington, DC: Office of Water.

U.S. Environmental Protection Agency (USEPA). 2002b. Wetland monitoring and assessment, a technical framework. EPA 843-F-02-002(h). Washington, DC: Office of Water.

U.S. Fish and Wildlife Service (USFWS). 1980a. Ecological services manual (101-104 ESM. Washington, DC: U.S. Department of the Interior, Fish and Wildlife Service, Division of Ecological Services.

U.S. Fish and Wildlife Service (USFWS). 1980b. Habitat Evaluation Procedures, Ecological Services Manual 102. Washington, DC.

U.S. Fish and Wildlife Service (USFWS). 1980c. Habitat Evaluation Procedures (HEP) Manual (103 ESM). Washington, DC.

U.S. Fish and Wildlife Service (USFWS). 1981. Implementation of the habitat evaluation procedures. FWS/DES-ESM 101. Washington, DC.

U.S. Forest Service. 1984. Field instructions for southern New England. Radnor, PA.

U.S. Geological Survey (USGS). 1982. Hydrologic Unit Map of the United States. Map scale (1:7,500,000). Washington, DC: U.S. Government Printing Office.

Waide, J. B., and J. R. Webster. 1976. Engineering systems analysis: applicability to ecosystems. Vol. 4. New York: Academic Press.

Wakeley, J. S. 1999. Case study: Application of the HGM Western Kentucky low-gradient riverine guidebook to monitoring of wetland development. TN WRP WG-EV-2.3. Vicksburg, MS: U.S. Army Engineer Research and Development Center. 
Wakeley, J. S., J. A. Barlow, and W. R. Bunkley. 2001. Functional assessment using the Hydrogeomprphic (HGM) approach: Applying the wet pine flats guidbook in the Southeast. ERDC TN-WRAP-01-04. Vicksburg, MS: U.S. Army Research and Development Center.

Walters, C. J., J. S. Collie, and T. Webb. 1988. Experimental designs for estimating transient responses to management disturbances. Canadian J ournal of Fisheries and Aquatic Sciences 45 (3):530-538.

Warry, N., and M. Hanau. 1993. The use of terrestrial ecoregions as a regional-scale screen for selecting representative reference sites for water quality monitoring. Environmental Management 17 (2):267-276.

Welcomme, R. L. 1979. Fisheries ecology for floodplain rivers. London, UK: Longman Publishers.

Westman, W. E. 1985. Ecology, impact assessment, and environmental planning. Vol. 2: Wiley-Interscience.

Wharton, C. H. 1978. The natural environments of Georgia. Atlanta, GA: Geologic and Water Resources Division and Resource Planning Section, Office of Planning and Research, Georgia Department of Natural Resources.

Whigham, D., A. Jacobs, D. Weller, T. Jordan, M. Kentula, S. Jensen, and D. Stevens. 2007. Combining HGM and EMAP procedures to assess wetlands at the watershed scale - status of flats and non-tidal riverine wetlands in the Nanticoke River watershed, Delaware and Maryland (USA). Wetlands 27 (3):462-478.

Whittier, T. R., R. M. Hughes, J. L. Stoddard, G. A. Lomnicky, D. V. Peck, and A. T. Herlihy. 2007. A structured approach for developing indices of biotic integrity: Three examples from streams and rivers in the western USA. Transactions of the American Fisheries Society 136 (3):718-735.

Winter, T. 1981. Uncertainties in estimating the water balance of lakes. Water Resource Bulletin 17(1):82-115.

World Wildlife Fund. 1992. Statewide wetlands strategies: A guide to protecting and managing theresource. Washington, DC: Island Press.

Zar, J. H. 1996. Biostatistical analysis. 3rd ed. Englewood Cliffs, NJ: Prentice Hall, Inc. 


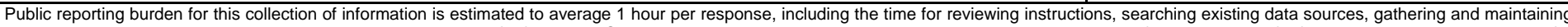

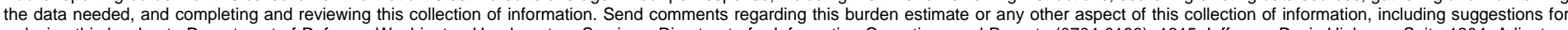

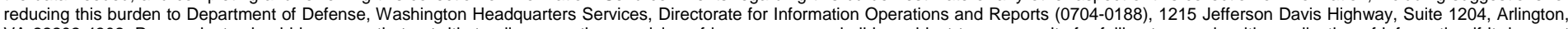

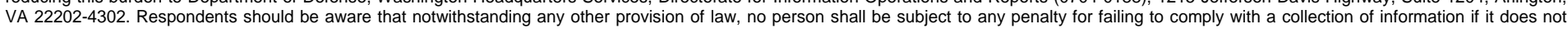
display a currently valid OMB control number. PLEASE DO NOT RETURN YOUR FORM TO THE ABOVE ADDRESS.
1. REPORT DATE (DD-MM-YYYY)
2. REPORT TYPE
3. DATES COVERED (From - To)

Final

June 2013

Hydrogeomorphic (HGM) Approach to Assessing Wetland Functions: Guidelines for

Developing Guidebooks (Version 2)

5a. CONTRACT NUMBER

5b. GRANT NUMBER

5c. PROGRAM ELEMENT NUMBER

\section{AUTHOR(S)}

R. Daniel Smith, Chris V. Noble, and Jacob F. Berkowitz

5d. PROJECT NUMBER

5e. TASK NUMBER

5f. WORK UNIT NUMBER

\section{PERFORMING ORGANIZATION NAME(S) AND ADDRESS(ES)}

8. PERFORMING ORGANIZATION REPORT NUMBER

Environmental Laboratory

U.S. Army Engineer Research and Development Center

ERDC/EL TR-13-11

3909 Halls Ferry Rd.

Vicksburg, MS 39180-6199

9. SPONSORING I MONITORING AGENCY NAME(S) AND ADDRESS(ES)

U.S. Army Corps of Engineers

Washington, DC 20314-1000

10. SPONSOR/MONITOR'S ACRONYM(S)

11. SPONSOR/MONITOR'S REPORT NUMBER(S)

\section{DISTRIBUTION I AVAILABILITY STATEMENT}

Approved for public release; distribution is unlimited.

\section{SUPPLEMENTARY NOTES}

\section{ABSTRACT}

The Hydrogeomorphic (HGM) Approach for assessing wetlands was developed by the U.S. Army Corps of Engineers as a procedure for assessing the capacity of a wetland to perform functions. The Approach requires classification of wetlands based on geomorphic setting, water source, and hydrodynamics. The objective of the Guidelines for Guidebook Development is to provide detailed guidance on methods and procedures that have proven helpful in developing existing guidebooks. This document contains an overview of the HGM Approach; how to classify and characterize wetland subclasses; developing assessment models; selecting reference wetlands and managing reference wetland data; testing and calibrating models; developing assessment protocols for using assessments; validating assessment models; and assessment application examples.

15. SUBJECT TERMS

(see reverse)

\section{SECURITY CLASSIFICATION OF:}

a. REPORT

UNCLASSIFIED

b. ABSTRACT
UNCLASSIFIED

c. THIS PAGE

UNCLASSIFIED
17. LIMITATION OF ABSTRACT
18. NUMBER OF PAGES

180 19a. NAME OF RESPONSIBLE PERSON

19b. TELEPHONE NUMBER (include area code) 
15. SUBJECT TERMS (concluded)

404 Regulatory Program

Assessment

Classification

Clean Water Act

Evaluation

Function

Functional assessment

Hydrogeomorphic Approach

Hydrology

Impact analysis

Indicators

Mitigation

Model

National Action Plan

Procedure

Reference wetlands

Wetland subclass 